\title{
A WEB ENGINEERING APPROACH FOR THE DEVELOPMENT OF BUSINESS PROCESS-DRIVEN WEB APPLICATIONS
}

A thesis submitted in partial fulfilment of the requirements for the degree of Doctor of Philosophy in Computer Science

\section{Victoria Torres Bosch}

\section{Supervisor: \\ Dr. Vicente Pelechano Ferragud}

UNIVERSIDAD POLITECNICA DE VALENCIA 


\title{
A Web Engineering Approach for the Development of Business- Process Driven Web Applications
}

\author{
Victoria Torres Bosch
}

Department of Information Systems and

Computation

Technical University of Valencia

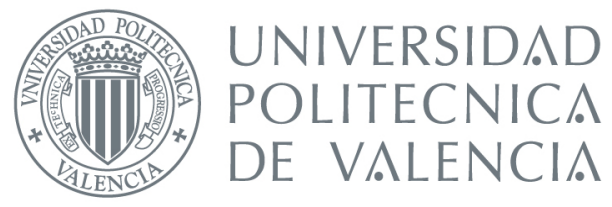

A thesis submitted in partial fulfilment of the requirements for the degree of Doctor of Philosophy in Computer Science

Supervisor: Dr. Vicente Pelechano Ferragud

July 2008 

Members of the Thesis Committee:

Dr. D. Oscar Pastor López

Catedrático de Universidad

Universidad Politécnica de Valencia

Dr. D. Joan Fons Cors

Colaborador

Universidad Politécnica de Valencia

Dr. D. Ernest Teniente López

Titular de Universidad

Universidad Politécnica de Catalunya

Dr. D. Antonio Ruiz Cortés

Titular de Universidad

Universidad de Sevilla

Dr. D. João Falcão e Cunha

Professor Associado

Universidade do Porto 



\section{Abstract}

Nowadays, the World Wide Web is established as the most common platform for the execution of corporate applications. These applications are called Web applications and among other functionalities, these must provide support for the Business Processes (BP) defined by the corporations.

From the Web Engineering area, different methods have proposed a solution to deal with the challenge of building this kind of Web applications (hereafter BP-driven Web applications). However, these solutions were mainly focused on the support for light-weight BPs (i.e. the check out process usually provided in on-line stores). In addition, other types of BPs where different participants (humans and systems) cooperate to accomplish a particular goal have not been properly addressed.

This thesis presents a Web Engineering method for the systematic specification and automatic generation of Web applications supporting BPs, understanding BPs in a wider sense, not limiting to the already addressed light-weight BPs. The proposed method provides mechanisms that allow specifying the particular characteristics of BP-driven Web applications at the modelling level (in a technological independent manner). In addition, based on the Model Driven Engineering, application specifications are combined and 
transformed to obtain different software artefact. On the one hand, by means of model-to-model transformations, we combine model specifications to obtain different models. On the other hand, by means of model-to-text transformations, models can be derived into executable code implemented in a particular technology. In addition, based on the reference model proposed for Workflow Management Systems, the method proposes the extension of the logical layer of the generated Web applications with the introduction of process engine.

The method proposed in this thesis is supported by a tool called BIZZY. This tool has been developed in the Eclipse environment and covers the development process from modelling to code generation. Specifically, the generated code corresponds to the Tapestry Web framework (framework for Java Web applications) and the WS-BPEL language, which allows the execution of the BP defined at the modelling level. 


\section{Resumen}

Actualmente, la World Wide Web se ha convertido en la plataforma más común para llevar a cabo el desarrollo de aplicaciones corporativas. Estas aplicaciones reciben el nombre de aplicaciones Web y entre otras funciones, deben de dar soporte a los Procesos de Negocio (PN) definidos por las corporaciones.

Esta tesis presenta un método de Ingeniería Web que permite el modelado y la construcción sistemática de aplicaciones Web que soportan la ejecución de PN. En este trabajo se conciben los PN desde un punto de vista más amplio que el abordado por otros métodos de Ingeniería Web. El tipo de PN abordados incluye tanto procesos cortos como largos. A grosso modo, esta concepción más amplia permite considerar procesos que involucran diferentes participantes (personas y/o sistemas) los cuales cooperan para llevar a cabo un objetivo particular. Además, dependiendo del tipo de proceso que se esté ejecutando (corto o largo), la interacción del usuario con el sistema deberá adaptarse a cada caso.

El método presentado en esta tesis ha sido desarrollado basándose en el Desarrollo de Software Dirigido por Modelos. De esta forma, el método propone un conjunto de modelos que permiten representar los diferentes aspectos que caracterizan las aplicaciones Web que soportan la ejecución de PN. Una vez 
el sistema ha sido representado en los modelos correspondientes, mediante la aplicación de transformación de modelos se obtiene otros modelos (transformaciones de modeloa-modelo) e incluso el código que representa el sistema modelado en términos de un lenguaje de implementación (transformaciones de modelo-a-texto).

El método propuesto en esta tesis está soportado por una herramienta llamada BIZZY. Esta herramienta ha sido desarrollada en el entorno de Eclipse y cubre el proceso de desarrollo desde la fase de modelado hasta la generación de código. En particular, el código generado corresponde con el framework Web Tapestry (framework que genera aplicaciones Web en Java) y con WS-BPEL, lenguaje que permite la ejecución de los $\mathrm{PN}$ definidos a nivel de modelado. 


\section{Resum}

Actualment, la World Wide Web s'ha convertit en la plataforma més comuna per a portar a terme el desenvolupament d'aplicacions corporatives. Aquestes aplicacions reben el nom d'aplicacions Web i entre altres funcions, deuen donar suport als Processos de Negoci (PN) definits per les corporacions.

Aquesta tesi presenta un mètode d'Enginyeria Web que permet el modelatge i la construcció sistemàtica d'aplicacions Web que suporten l'execució de PN. En aquest treball es conceben els PN des d'un punt de vista més ampli que el considerat per altres mètodes d'Enginyeria Web. El tipus de PN abordats inclou tant processos curts com llargs. En general, aquesta concepció més àmplia permet considerar processos que involucren diferents participants (persones i/o sistemes) els quals cooperen per a portar a terme un objectiu particular. A més, depenent del tipus de procés que s'estigui executant (curt o llarg), la interacció de l'usuari amb el sistema haurà d'adaptar-se a cada cas.

El mètode presentat en aquesta tesi ha estat desenvolupat basant-se en el Desenvolupament de Programari Dirigit per Models. D'aquesta forma, el mètode proposa un conjunt de models que permeten representar els diferents aspectes que caracteritzen les aplicacions Web que suporten l'execució de 
PN. Una vegada el sistema ha estat representat en els models corresponents, mitjançant l'aplicació de transformació de models s'obtenen altres models (transformacions de model-amodel) i fins i tot el codi que representa el sistema modelat en termes d'un llenguatge d'implementació (transformacions de model-a-text).

El mètode proposat en aquesta tesi està suportat per una eina cridada BIZZY. Aquesta eina ha estat desenvolupada en l'entorn d'Eclipse i cobreix el procés de desenvolupament des de la fase de modelatge fins a la generació de codi. En particular, el codi generat correspon amb el framework Web Tapestry (framework que genera aplicacions Web en Java) i amb WS-BPEL, llenguatge que permet l'execució dels PN definits a nivell de modelatge. 
A mi hermana Gloria 



\section{Acknowledgements/Agradecimientos}

Esta tesis es el fruto de muchas discusiones y horas de trabajo, horas que he compartido con mucha gente y que por eso hago esta tesis tan suya como mía.

A mi director de tesis el Dr. Vicente Pelechano, por compaginar como nadie los roles de director y amigo. Por haberme dado la oportunidad de iniciarme y crecer en el fascinante mundo de la investigación. Sin duda alguna, tu calidad investigadora unida a tu calidad humana hacen de ti el mejor patrón para llevar con éxito una empresa como ésta.

Al Dr. Oscar Pastor por los esfuerzos constantes que siempre has realizado, en función de director pero en actitud de amigo.

A Mati por tu amistad, ayuda y tus valiosos consejos ofrecidos durante todo este tiempo.

A Joan y Manoli, por la ayuda incondicional y el cariño que siempre me habéis mostrado así como de vuestra experiencia investigadora.

A Marta, Pedro, Javi, Ricardo y Gonzalo por todos los momentos que hemos pasado juntos a lo largo de esta experiencia. 
A Pau, por el esfuerzo y dedicación que siempre has demostrado para hacer realidad las ideas que se desarrollaron en esta tesis.

A Bea y Giovanni, por traer la alegría a nuestro laboratorio y por darme una lección de prioridades en la vida.

A Ana, por el valioso ejemplo que nos das cada día.

A Paco por el esfuerzo que has hecho para que OOWS sea una realidad.

A Carlos y Fani por el cariño y la amistad que me habéis demostrado.

Al resto de miembros del grupo OO-Method (Nathalie, Paqui, Sonia, Isabel, Juan, Jorge, Sergio, Ignacio, Jose Luis, Luis y Nelly) por ser unos fantásticos compañeros de abordo.

A Josep por el apoyo constante que me has demostrado y por el ejemplo de capacidad de trabajo que me has dado.

A mis padres, Gloria y Paco, pues todo lo que tengo se lo debo a ellos. Sin duda alguna, su cariño y apoyo a lo largo de toda mi vida ha permitido que llegara hasta aquí.

A mi hermana Gloria, por su apoyo incondicional y la confianza que siempre ha demostrado tener en mí. 
A Alberto, por la paciencia que ha demostrado a lo largo de estos años.

A mi hermano Fran y a $M^{a}$ José por regalarnos a Francisco, la mejor terapia contra el estrés que nunca se haya inventado. 



\section{Table of Contents}

1 INTRODUCTION

1.1 Purpose

1.2 Problem Statement

1.3 MAIN CONTRIBUTIONS

1.4 RESEARCH METHODOLOGY

1.5 THESIS DEVELOPMENT CONTEXT

1.6 STRUCTURE OF THE THESIS
31

33

38

39

42

44

45

49

2 FOUNDATIONS

50

2.1.1 THE OOWS Web ENGINEERING Method 50

2.2 Business Processes

2.2.1 BPMN: BUSINESS PROCESS MODELLING NOTATION 61

2.2.2 WS-BPEL: BUSINESS PROCESS EXECUTABLE LANGUAGE FOR WEB SERVICES

2.3 Business Process-Driven Web APPlications 72

2.3.1 SHORT-RUNNING BUSINESS PROCESSES 73

2.3.2 LONG-RUNNING BUSINESS PROCESSES 76

2.3.3 REQUIREMENTS FOR DEALING WITH BP-DRIVEN WEB APPLICATIONS 80

2.4 CONCLuSIONS

82

3 STATE OF THE ART 85 
3.1 Web ENGINEERING AREA

$\begin{array}{lll}3.1 .1 & \text { UWAT }+ & 87\end{array}$

3.1.2 WebML: Web Modeling Language 91

3.1.3 OOHDM: OBJECT-ORIENTED HYPERMEDIA DESIGN METHOD 96

3.1.4 UWE: UML BASED-WEB ENGINEERING 100

3.1.5 OO-H: OBJECT-ORIENTED HYPERMEDIA METHOD 104

3.1.6 WSDM: WeB Site DESIGN METHOD 106

$\begin{array}{lll}3.1 .7 & \text { HERA } & 109\end{array}$

3.1.8 MIDAS 112

3.1.9 DISCUSSION 115

$\begin{array}{lll}3.2 & \text { CONCLUSIONS } & 123\end{array}$

4 DEVELOPMENT PROCESS 125

$\begin{array}{lll}4.1 & \text { INTRODUCTION } & 125\end{array}$

4.1.1 USING BPMN to DeFINE THE DEVElopMent Process 127

4.2 The Big Picture 128

4.3 The MODELING STEP 132

4.3.1 The OO-Method Modeling SteP 132

4.3.2 THE SERVICES MODEL 133

4.3.3 The Business PROCESS Model 134

4.3.4 The OOWS Method MOdELING SteP 134

4.4 The CODE GENERATION STEP 135

4.4.1 WS-BPEL CODE GENERATION STEP 135

4.4.2 USER INTERFACE CODE GENERATION STEP 137

4.5 CONCLUSIONS 138

$\underline{5}$ BUSINESS PROCESS MODEL 141

5.1 BUSINESS PROCESS MODEL BASED ON THE BPMN NOTATION 142 
5.2 BPMN LIMITATIONS TO SUPPORT THE BPM

5.3 PHD TheSIS DELIVERY EXAMPLE 145

5.4 BPMN EXTENSIONS 148

5.4.1 DifFERENTIATING HUMAN PARTICIPANT BEHAVIOUR 149

5.4.2 DEFINING THE FUNCTIONALITY OF EACH PROCESS TASK 150

5.4.3 LANE DEPENDENCES 152

5.5 CONCLUSIONS 153

6 NAVIGATIONAL MODEL EXTENSION

6.1 INTRODUCTION 156

6.2 Introducing the NeW Navigational Primitives 157

6.3 NaVigational Primitives to SuPPORT BP EXeCUTION 160

6.3.1 PROCESS CONTEXT 160

6.3.2 ACTIVITY CONTAINER 165

$\begin{array}{lll}\text { 6.3.3 MAIN-AIU } & 170\end{array}$

6.3.4 HUMAN-AIU 173

6.3.5 COMPLEMENTARY-AIU 176

$\begin{array}{lll}\text { 6.3.6 INDEX } & 179\end{array}$

6.3.7 ClaSs-VIEW 184

6.3.8 SERVICE-DATA-VIEW 186

6.3.9 SERVICE-FUNCTIONAL-VIEW 188

$\begin{array}{lll}\text { 6.3.10 PROCESS-LINK } & 191\end{array}$

6.4 EXTENDED NAVigational METAMOdEL 195

6.5 CONClusions 196

7 PRESENTATION MODEL EXTENSION 197

7.1 Introducing the New Presentation Primitives 197

7.2 PRESENTATION PRIMITIVES TO SUPPORT BP EXECUTION 198 
7.2.2 LIST BUILDER PATTERN 202

7.3 EXtended Presentation Metamodel 204

7.4 DEALING WITH USABILITY ISSUES 205

7.4.1 PREVENTING INPUT ERRORS 207

7.4.2 Displaying The TASKS PENDING TO COMPLETE 210

7.4.3 NOTIFY USERS WHEN THEY ARE EXPECTED TO PERFORM A TASK 212

$\begin{array}{lll}7.5 & \text { CONCLUSIONS } & 213\end{array}$

$\underline{8}$ ARCHITECTURAL EXTENSION

$\begin{array}{lll}\text { 8.1 INTRODUCTION } & 216\end{array}$

$\begin{array}{lll}\text { 8.2 EXTENSION OVERVIEW } & 217\end{array}$

8.2.1 PRESENTATION LAYER 221

8.2.2 BUSINESS TIER 222

8.2.3 DATA TIER 223

8.3 DEALING WITH HUMAN TASKS 223

8.3.1 Task Manager Web SERVICE 223

8.3.2 WEB SERVICE CONVERSATION WHILE HuMAN PARTICIPATION 228

8.4 CONCLUSIONS 230

9 MODEL TRANSFORMATIONS $\quad 231$

9.1 The Big Picture 232

9.2 MODEL TRANSFORMATIONS TO GENERATE WS-BPEL CODE 235

9.2.1 FROM THE BPM TO BPMN ACCORDING TO THE BABEL TOOL 238

9.2.2 COMPLETING THE WS-BPEL DOCUMENT 243

9.2.3 XSD \& WSDL GENERATION 252

9.3 MODEL TRANSFORMATIONS TO GENERATE THE USER INTERFACE 255 
9.3.1 BPMN TO OOWS NAVIGATIONAL MODEL 255

9.3.2 CODE GENERATION FOR A WEB FRAMEWORK 259

9.4 Conclusions $\quad 263$

10 TOOL SUPPORT

10.1 TOOL OVERVIEW 266

10.2 From A BP SPECIFICATION TO A WEb APPLICATION: STEP BY

STEP 269

10.2.1 STEP 1: SYSTEM SPECIFICATION 271

10.2.2 SteP 2: GENERATION OF THE WEB SySteM NAVIGATION 274

10.2.3 SteP 3: GenerATION OF THE WEB SySteM GUI 275

10.2.4 STEP 4: GENERATION OF WS-BPEL EXECUTABLE BPS 279

10.3 DEPLOYMENT 281

10.3.1 WEB APPLICATION DEPLOYMENT 282

10.3.2 WS-BPEL DEPLOYMENT 283

10.4 CONCLUSIONS 285

11 CONCLUSIONS \& FURTHER WORK

11.1 MAIN CONTRIBUTIONS 287

11.2 FURTHER WORK 289

$\begin{array}{lll}11.3 & \text { Publications } & 291\end{array}$

A LYBRARY4U CASE STUDY

A.1 DESCRIPTION 295

A.2 Structural Model 296

A.3 SERVICES MODEL 298

A.4 USER MODEL 299 
A.5 Anonymous Navigational ANd Presentation Model 300

$\begin{array}{lll}\text { A.5.1 AUTHORING-IN-THE-LARGE } & 301\end{array}$

B SHORT-RUNNING BP CASE STUDY $\quad 313$

B.1 DESCRIPTION 313

B.2 Structural Model $\quad 314$

B.3 SERVICES MODEL

B.4 BuSINESS PROCESS MODEL 316

B.5 NaVigational MODEL 316

$\begin{array}{lll}\text { B.5.1 AUTHORING-IN-THE-LARGE } & 317\end{array}$

$\begin{array}{lll}\text { B.5.2 AUTHORING-IN-THE-SMALL } & 317\end{array}$

B.6 USABILITY ASPECTS 320

$\underline{C}$ LONG-RUNNING BP CASE STUDY

C.1 DESCRIPTION

C.2 Structural Model

C.3 SerVices MOdel 323

C.4 Business Process Model 324

C.5 Navigational and Presentation Models 325

C.5.1 Member Navigational AND Presentation Model 326

C.5.2 SECRETARY NAVIGATIONAL MODEL 330

$\begin{array}{lll}\text { C.5.3 LIBRARIAN NAVIGATIONAL MODEL } & 336\end{array}$ 


\section{List of Figures}

Figure 1.1 Workflow Reference Model from (Hollingsworth, 1995)

Figure 1.2 Research methodology followed in this thesis 42

Figure 2.1 Types of Users According to the Different Access Permission. .52

Figure 2.2 Example of User Specialization ................................. 52

Figure 2.3 Navigational Map for the Library4U Case Study ....... 53

Figure 2.4 Navigational Context .......................................... 56

Figure 2.5 Layout Patterns applied to Navigational Class and

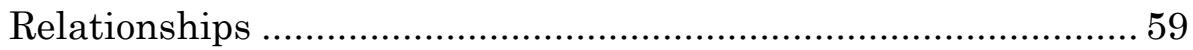

Figure 2.6 Events Graphical Elements ...................................... 62

Figure 2.7 Activities Graphical Elements ................................... 63

Figure 2.8 Gateways Graphical Elements ................................ 64

Figure 2.9 Connecting Objects Graphical Elements.................... 65

Figure 2.10 Swimlanes Graphical Elements.............................. 65

Figure 2.11 Artifacts Graphical Elements .................................. 66

Figure 2.12 Relation between WS-BPEL Process Definition and

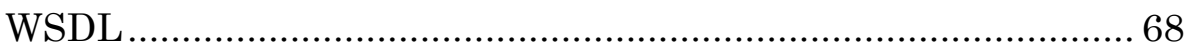

Figure 2.13 Partner Link Type structure................................... 69

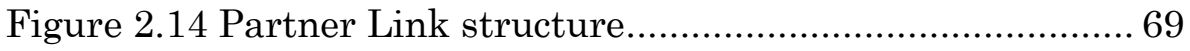

Figure 2.15 Example of a short-running process: Checkout Process

Figure 2.16 Web interface to complete the first activity of the

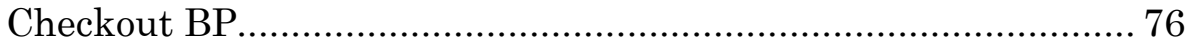


Figure 2.17 Example of a long-running process: Book Purchase Request.

Figure 2.18 Web interface to complete the "Request Validation" activity of the long-running $\mathrm{BP}$ 79

Figure 3.1 UWAT+ Development Process .................................. 91

Figure 3.2 Data Model Extended.................................................. 94

Figure 3.3 WebML Extended Development Process .................... 95

Figure 3.4 MIDAS development process focused on BP ............ 115

Figure 4.1 Process Development ............................................. 130

Figure 4.2 Expanded Sub-process System Specification ............ 131

Figure 4.3 Expanded Sub-process Web Specification ................. 132

Figure 4.4 Expanded Sub-process WS-BPEL generation ........... 136

Figure 4.5 Expanded Sub-process Tapestry Web framework

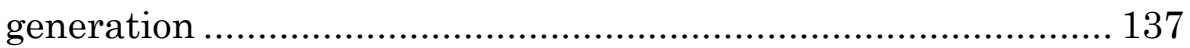

Figure 5.1 PhD Thesis Delivery BP......................................... 146

Figure 5.2 Excerpts of the Structural Model and Business Process

Diagram 147

Figure 5.3 Metamodel Architecture ........................................ 148

Figure 5.4 Extension Defined to the BPMN Metamodel ............ 149

Figure 6.1 Navigational Map for the Secretary User type (Authoring-in-the-large)

Figure 6.2 Book Purchase Request Process Context (Authoring-inthe-small) 158

Figure 6.3 Navigational Map for the Member User type (Authoring-in-the-large)

Figure 6.4 Check Out Process Context (Authoring-in-the-small)

Figure 6.5 Web page corresponding to the "Processes" Section.. 162 
Figure 6.6 Web page corresponding to the "Shopping Cart"

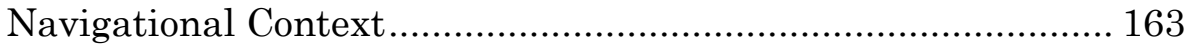

Figure 6.7 Process-Context primitive........................................ 163

Figure 6.8 Metamodel Excerpt including the Process Context primitive. 164

Figure 6.9 Web page corresponding to the "Validate Request" Activity Container. 166

Figure 6.10 Activity-Container primitive................................ 167 Figure 6.11 Metamodel Excerpt including the Activity Container primitive 169

Figure 6.12 Main-AIU primitive 171

Figure 6.13 Metamodel Excerpt including the Main-AIU primitive 172

Figure 6.14 Implementation of a Human-AIU.......................... 174

Figure 6.15 Human-AIU primitive......................................... 175

Figure 6.16 Metamodel Excerpt including the Human-AIU

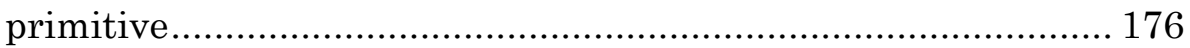

Figure 6.17 Complementary-AIU primitive .............................. 177 Figure 6.18 Metamodel Excerpt including the Complementary-

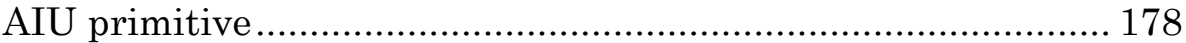

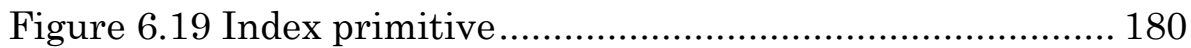

Figure 6.20 Implementation of a Main-AIU when the index gets

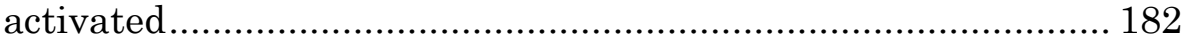

Figure 6.21 Implementation of a Main-AIU when the index gets deactivated 183

Figure 6.22 Metamodel Excerpt including the Process-Index primitive. 184

Figure 6.23 Class-View primitive 185 
Figure 6.24 Metamodel Excerpt including the NavigationalClass primitive 186

Figure 6.25 Service-View primitive 187

Figure 6.26 Metamodel Excerpt including the Service-data-view primitive. 188

Figure 6.27 Web page corresponding to the "Payment" Activity Container 189

Figure 6.28 Service-Functional-View primitive 190 Figure 6.29 Metamodel Excerpt including the Service-functionalview 191 Figure 6.30 Detail of a Navigational Context including a processlink 192

Figure 6.31 Web page corresponding to the Shopping Cart Navigational context. 193

Figure 6.32 Process-Link primitive 194

Figure 6.33 Metamodel Excerpt including the Process link primitive. 194

Figure 6.34 Navigational Model Excerpt including the BP related primitives 195

Figure 7.1 Presentation attributes associated to the Book Purchase Request BP................................................................ 198 Figure 7.2 Details on Demand Pattern in use .......................... 200 Figure 7.3 Detail On Demand Pattern..................................... 201 Figure 7.4 Metamodel Excerpt including the Details on Demand

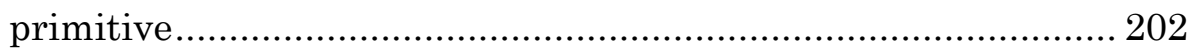

Figure 7.5 List Builder Pattern in use .................................... 203 Figure 7.6 Metamodel Excerpt including the List Builder primitive 204

Figure 7.7 Presentation Metamodel 205 
Figure 7.8 Models Used for the generation of Web Pages .......... 211

Figure 7.9 Generated User Interface for a Short-running BP.... 212

Figure 7.10 Modified version of a BP definition......................... 213

Figure 8.1 Three Layer architecture for Process-driven Web

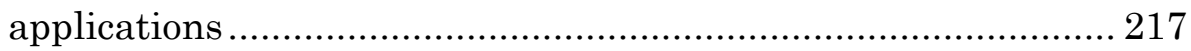

Figure 8.2 Tapestry File Organization.................................... 218

Figure 8.3 Task Manager Service Interface.............................. 223

Figure 8.4 WSDL for the Task Manager Service ....................... 224

Figure 8.5 Task Manager Structure........................................ 225

Figure 8.6 Role of the Task Manager Web service..................... 228

Figure 9.1 Transformations Defined for generating the WS-BPEL code...... 232

Figure 9.2 Transformations Defined for generating the Web site for the Tapestry Framework. 233

Figure 9.3 Interaction between the WS-BPEL process and the set

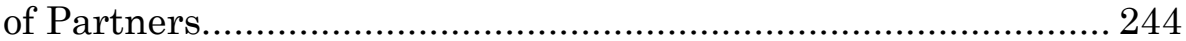

Figure 9.4 Basic Process Context Generated for the Secretary role 258

Figure 9.5 Web Application Abstraction Levels 261

Figure 10.1 Tool Support for the OOWS-BP Development Process 267

Figure 10.2 STP BPMN Modeller. 271

Figure 10.3 Different Editors provided by the OLIVANOVA Modeller 272

Figure 10.4 Tree-based EMF editor for the Services Model ....... 273 Figure 10.5 Tree-based EMF editor for the Extension defined over the BPMN notation................................................................. 274

Figure 10.6 From BPM to OOWS Navigational Model.............. 275

Figure 10.7 M2T Transformations .......................................... 276 
Figure 10.8 Web application folder within the TOMCAT Web

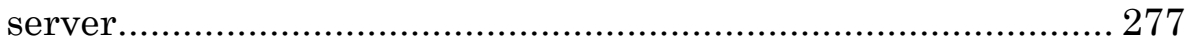

Figure 10.9 WEB-INF Folder Structure ................................ 278

Figure 10.10 M2M transformation to prepare BP definitions

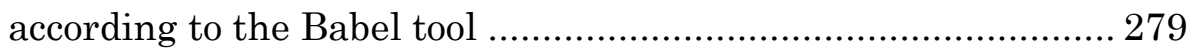

Figure 10.11 Generation of a partial WS-BPEL BP ................. 280

Figure 10.12 Generation of a complete WS-BPEL BP ............... 280

Figure 10.13 Generation of the Interface and Data types used by

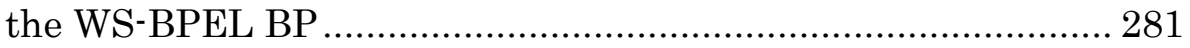

Figure 10.14 ActiveBPEL prepared environment..................... 284

Figure 10.15 Active-bpel folder within the TOMCAT Web server 284

Figure A.1 Library4U Class Diagram ...................................... 297

Figure A.2 User Model............................................................. 300

Figure A.3 Anonymous User Navigational Map ........................ 301

Figure A.4 Home Navigational Context ................................... 302

Figure A.5 Home Navigational Context (Presentation proprieties) 303

Figure A.6 Implemented Home Navigational Context ............... 304

Figure A.7 Books Navigational Context................................... 305

Figure A.8 Books Navigational Context (Presentation proprieties) 306

Figure A.9 Anonymous Book Navigational Context .................. 307

Figure A.10 Anonymous Book Navigational Context (Detail) .... 308

Figure A.11 Authors Navigational Context .............................. 309

Figure A.12 Authors Navigational Context (Presentation

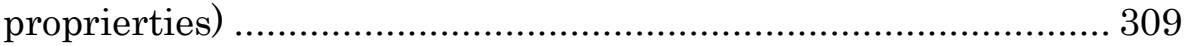

Figure A.13 Authors Navigational Context .............................. 310

Figure A.14 Shopping Cart Navigational Context..................... 311 
Figure A.15 Shopping Cart Navigational Context (Presentation

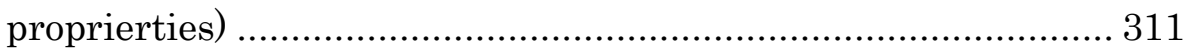

Figure A.16 Shopping Cart Web page ........................................ 312

Figure B.1 Structural Model ..................................................... 314

Figure B.2 Business Process Model........................................... 316

Figure B.3 Registered User Navigational Map......................... 317

Figure B.4 Checkout Navigational Context .............................. 318

Figure B.5 Generated User Interface for the "Shipping" task.... 319

Figure B.6 Generated User Interface for the "Payment" task.... 319

Figure B.7 Generated User Interface for the "Gift wrap" task... 320

Figure C.1 Excerpt of the Structural Model............................... 323

Figure C.2 Business Process Model........................................... 325

Figure C.3 Member Navigational Map..................................... 326

Figure C.4 Home page including the Processes Link ................ 327

Figure C.5 My TODO List for a Secretary user .......................... 328

Figure C.6 RBP Process Context for the Member user............... 329

Figure C.7 Request Book Purchase task for the member user... 329

Figure C.8 Secretary User Navigational Map........................... 330

Figure C.9 Details of the Request Book Purchase Process Context 331

Figure C.10 RBP Process Context for the Secretary user........... 332 Figure C.11 RBP Process Context for the Secretary user (Presentation properties) 333

Figure C.12 List Build pattern applied to the Request Validation Activity Container 334

Figure C.13 Detail On Demand pattern applied to the Request Validation Activity Container 335

Figure C.14 Generated interface for the "Pick Up Books" activity 
Figure C.15 Librarians Navigational Map............................... 336

Figure C.16 Loan book Navigational Context........................... 337

Figure C.17 Generated interface for the "Loan Book" activity ... 338 


\section{Chapter 1}

\section{Introduction}

Initially, in the early nineties, the World Wide Web (WWW) was basically used as a medium to get information (Berners-Lee, 1996). Fortunately, however, the evolution that the WWW has undergone makes now a broader use of it. In fact, the WWW has been established as the common platform for the development of a vast amount of software systems (Ginige \& Murugesan, 2001b). These days it is possible to find Web applications for almost every domain, i.e., economy, industry, education, health, public administration, leisure, etc. Moreover, as was reported in (Kappel et al., 2006), within each of these domains, the complexity that Web applications can reach varies in accordance with historical evolution. The different categories of Web applications that have appeared since the beginning of the Web are document-centric, interactive, transactional, workflow-based, collaborative, portaloriented, ubiquitous, social and semantic. Although all these kinds of applications share multiple characteristics, each one has its own application field. Therefore, these applications require a special analysis for their adequate development.

Specifically, the work presented in this thesis has been developed in the context of Web applications that are included within the 
workflow-based ${ }^{1}$ category (hereafter BP-driven Web applications). Examples of these systems are on-line stores or corporate web systems that support well-defined processes such as incidence management, material purchases, etc. Throughout this work, we propose to further the development of systems of this kind by combining the Web Engineering field (WE) (Murugesan \& Ginige, 2001) and the Model Driven Engineering field (MDE) (Selic, 2003; Schmidt, 2006).

With regard to the WE field, we propose a set of mechanisms to improve WE methods to properly represent systems of this kind at the modelling level. These mechanisms span different concerns. On the one hand, we have introduced a model (the Business Process Model -BPM) into the development process to capture Business Process (BP) requirements properly. On the other hand, a set of new abstractions related to user interaction have been defined in order to improve the user experience during BP execution. These new abstractions are associated to the Navigational and Presentation models of typical Web Engineering approaches (Murugesan \& Ginige, 2001b). Moreover, a review of the architecture of the applications generated by Web engineering methods is also performed. In fact, based on the architecture of Business Process Management Systems (BPMS), we propose introducing a process engine into the architecture of the generated Web applications.

${ }^{1}$ In this thesis, we use the term "business process" (BP) as a synonym of "workflow". 
With regard to the MDE field, we propose to follow a strategy that allows us to move the systems that are specified at the modelling level into a particular implementation. This strategy also allows us to combine different models in a previous stage of the development process in order to produce other models. For instance, by combining $\mathrm{BP}$ descriptions with structural and behavioural specifications we can generate the models that abstract the GUI which allows the original BP specifications to be executed.

The remainder of this chapter is organized as follows. Section 1.1 presents the reasons that have inspired this thesis work. Section 1.2 states the problems that we deal with in this work. Section 1.3 presents the solution to these problems which constitutes the main contribution of this work. Section 1.5 presents the context in which this work has been developed. Finally, section 1.6 presents the structure of the whole document, providing a brief description of each chapter.

\subsection{Purpose}

Nowadays, the WWW is considered to be the most common platform that is used for the development of software applications. When an organization needs a solution to handle its BPs, it is very common to deal with the problem from the WE field (Murugesan \& Ginige, 2001b). Several methods have been developed to cope with the systematic development of Web applications. Moreover, 
within this discipline, all of these methods have evolved according to the needs that have emerged as a consequence of the evolution of the WWW. Specifically, during the third edition of the International Workshop on Web-Oriented Software Technologies (IWWOST2), some of the most well-known approaches of WE provided a solution to deal with the integration of BPs. However, the separation of concerns that was proposed to differentiate pure navigation from BP navigation was not properly achieved. These proposals moved the flow of the BPs into the navigational model producing navigational models that were too complex to handle. Moreover, these solutions did not address the integration of $\mathrm{BP}$ in the broadest sense. These solutions only dealt with the integration of light-weight BPs. Due to this limitation, aspects such as the cooperation of different types of users to achieve a common goal, the consideration of manual tasks or handling multiple instances of a specific process are out of the scope of their proposed solutions. If we take the definition given by the Workflow Management Coalition in (WfMC, 1999), a workflow is defined as "the automation of a business process, in whole or part, during which documents, information or tasks are passed from one participant to another for action, according to a set of procedural rules". Therefore, BP-driven Web applications can be defined as systems that allow BPs to be handled within or between different enterprises. The aspects that characterize Web applications of this kind are the following:

\footnotetext{
${ }^{2}$ http://www.dsic.upv.es/ west/iwwost03/
} 
- Distributed Systems: We are not dealing with isolated systems where all the functionality is provided by a single system. In this case, different systems are integrated with each other and collaborate to achieve a common goal. This situation is very frequent within a Business-to-Business (B2B) environment where applications are not considered as monolithic systems and where the WWW is commonly used as the platform for their integration.

- Definition of Manual Tasks: The definition of a BP can involve the participation of automatic systems and human beings. In particular, the role that can be performed by people within the definition of a BP can be twofold:

1. As a participant inputting data to the process that performs a specific task (introduction of data through the use of an adequate user interface).

2. As a participant performing tasks that are not automated in the process (sending a fax, making a phone call, organizing a meeting, etc.).

- Temporal Constraints: The execution of a BP can extend in time for weeks, months or even years. For instance, a process can require the sequential participation of different participants, which may extend the process. Another example is when the definition of the process includes temporal constraints (starting, finishing or maximum duration dates), which can also extend the time required. 
- Kinds of interaction between the participants of a BP: Apart from the human-machine and machine-machine interaction that already exist in traditional applications, BPs can also take into account the human-human interaction (interaction that is commonly found in workflow applications).

In addition to considering Web engineering methods, Business Process Management Systems (BPMS) can also be taken into account as a solution for handling the BPs of the organization (in particular for long-running BPs). Examples of these systems are JBoss jBPM ${ }^{3}$, IBM WebSphere ${ }^{4}$, Oracle BPEL Process Manager ${ }^{5}$ or Biztalk 6 . These systems, according to the definition provided by the Workflow Management Coalition in (WfMC, 1999) are " $A$ system that defines, creates and manages the execution of workflows through the use of software, running on one or more workflow engines, which is able to interpret the process definition, interact with workflow participants and, where required, invoke the use of IT tools and applications". As this definition states, these tools provide support to the BPM within its whole life-cycle. This life-cycle includes five stages which are BP (1) Design, (2) Modelling, (3) Execution, (4) Monitoring and (5) Optimization. BPMS include different tools to perform each of these stages. For instance, while a graphical editor allows performing the modelling

\footnotetext{
${ }^{3}$ http://www.jboss.com/products/jbpm

${ }^{4}$ http://www-306.ibm.com/software/websphere/

${ }^{5}$ www.oracle.com/technology/bpel

${ }^{6} \mathrm{http}: / / \mathrm{www} . \mathrm{microsoft.com} /$ biztalk/
} 
stage, the process execution runtime allows activating processes and orchestrating people, data and systems that are involved in the BP. Figure 1.1 shows the typical components that are available in most BPMS products.

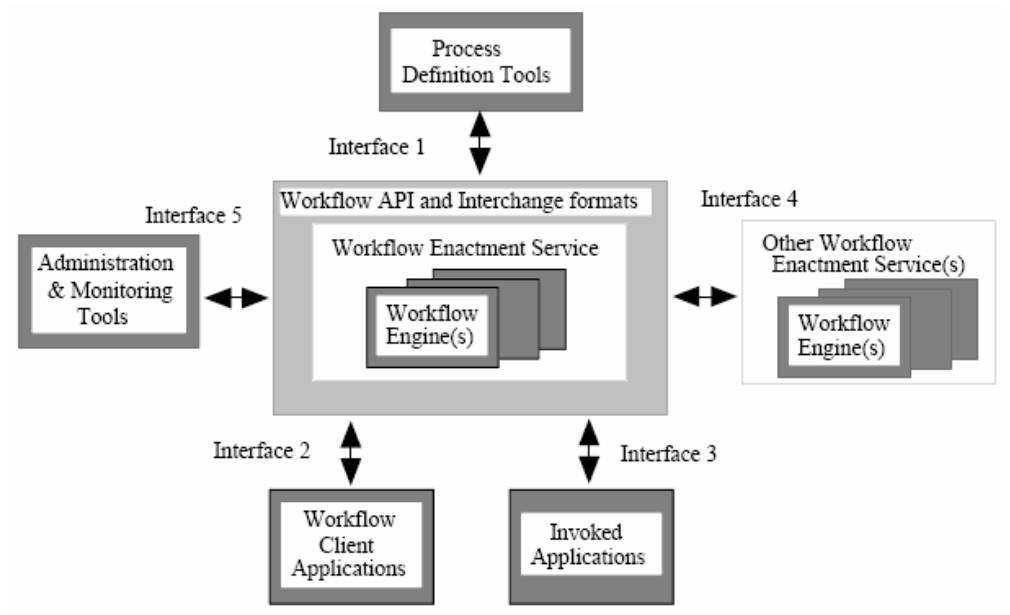

Figure 1.1 Workflow Reference Model from (Hollingsworth, 1995)

However, BPM systems are mainly focused on the process itself (its definition, execution and efficiency) and not in other aspects such as the user interfaces used to allow users interacting with the process. In fact, the kind of interfaces provided by these solutions are quite basic and little elaborated. Moreover, these interfaces are provided at the implementation level. As a result, any modification must be performed at this level. This approach does not fit in a context where the software development process is based on models. Moreover, the majority of these solutions are commercial suits whose use and implantation forces us to use proprietary languages and technologies, moving us away from the standards. 
To sum up, these two approaches (WE and BPMS) constitute different solutions for the execution of two different types of BPs, one for short-running 7 processes and another for long-running processes. On the one hand, WE methods deal with the integration of BPs into Web applications focusing only on short-running processes. Moreover, these methods attempt to carry out the integration by taking only navigational issues into account. As result, a poor separation of concerns regarding navigation and functionality is obtained. On the other hand, even though BPMS solutions provide support for handling long-running processes, they are highly technology dependent.

\subsection{Problem Statement}

As the previous section clearly indicates, the alternatives that are currently available to develop BP-driven Web applications fail to fulfil the necessary requirements. None of the solutions from either of these fields handles BPs that includes both types, shortrunning and long-running processes. Therefore, in order to develop applications of this kind, from an engineering point of view, the following research questions arise:

- Research question 1. What types of BPs should be considered when referring to BP-driven Web applications and how should they be addressed from the Web engineering perspective?

7 The term short-running is used as a synonym for light-weight processes. 
- Research question 2. Since WE is moving towards MDE (Vallecillo et al., 2007), what role should BP specifications play throughout the development process?

- Research question 3. How should BP specifications be defined during the development process (i.e. which notation/language)?

- Research question 4. How can a correct separation of concerns with regard to navigation be achieved when we are dealing with BP integration?

These research questions are analyzed and answered in the following section.

\subsection{Main Contributions}

The challenge of integrating BPs into Web applications has already been addressed by existing Web engineering methods. However, the proposed solutions do not perform the integration of BPs in their broader sense. Therefore, we provide a method for the systematic development of BP-driven Web applications for both short-running and long-running processes. We have taken into account the four research questions presented above in order to define the method. These questions represent a key factor in the successful achievement of the proposal.

First of all, regarding research question 1, we present a method that allows short-running and long-running BPs to be represented. Although BPs are similarly specified at the modelling 
level in both cases (both are represented as BP diagrams using a particular graphical notation), the GUIs that are generated to cope with their execution are different for each case. This differentiation produces a more appropriate GUI for each case. Besides, taking into account the characteristics that BP-driven Web applications require, the method also handles:

1. The definition of manual tasks within the BP specifications. The particularity of activities of this kind is that they are completely performed by human participants and outside of the boundaries of the system.

2. The definition of BPs as a composition of activities that are performed by different systems.

Regarding research question 2 , we propose the use of $\mathrm{BP}$ specifications to produce two different kinds of artefacts during the development process. Following the MDE, a set of model transformations (model-to-model and model-to-text) has been defined to automate as much as possible the production of the following artefacts as much as possible:

1. The Navigational and Presentation models required to support BP specified in the BPM. These models respectively capture the requirements in terms of (1) the content and functionality necessary to allow the user to perform process tasks and (2) the organization of these elements within the corresponding GUI.

2. An equivalent definition of the $\mathrm{BP}$ in terms of an executable language. This transformation will bring these 
specifications to life by deploying them into a process engine.

This set of transformations has been automated and is provided as part of a prototype tool ${ }^{8}$. This tool has been developed in the context of the Eclipse platform and supports the proposed method from the modelling phase through the code generation phase, thus automating the entire development process.

Regarding research question 3, we propose the use of standards during the development process. Thus, we propose the use of:

1. The BPMN (OMG, 2006) notation for specifying BPs at the modelling level.

2. The WS-BPEL (Andrews et al., 2003) language for the execution of these BPs.

Finally, regarding research question 4, we propose to achieve the separation of concerns related to navigation by maintaining the navigation that occurs during $\mathrm{BP}$ execution inside the definition of the process. This is possible since we have extended the architecture of the generated Web applications by introducing a process engine that is capable of handling the execution of BPs. This element is necessary at the architectural level since the navigation that occurs during BP execution (1) is more controlled ${ }^{9}$ than pure/content navigation and (2) the flow complexity found in long-running BPs cannot be handled properly in the Navigational

\footnotetext{
8 http://oomethod.dsic.upv.es/labs/projects/bizzy

9 The process is the one driving the user to the next step
} 
model. However, although we do not move BP flow into the Navigational model, we still need to specify the set of data and functionality that is going to be included in the Navigational models in order to perform BP tasks. Therefore, these are the kind of elements that are included in the Navigational model.

The solution proposed in this thesis has been applied to a method developed within the Web Engineering area, the OOWS (ObjectOriented Web Solution) approach (Fons, 2008). This approach has already been extended to cope with requirements (Valderas, 2008), adaptivity (Rojas, 2008), and Service Oriented Architectures (Quintero, 2008). In this thesis, we propose an extension to endow the method with enough expressivity to model Web applications that provide support to the execution of BPs.

\subsection{Research Methodology}

Taking as reference the design research methodology described by (March \& Smith, 1995; Vaishnavi \& Kuechler, 2004), the research performed in this thesis has involved the following five steps: (1) awareness of the problem, (2) suggestion, (3) development, (4) evaluation, and (5) conclusion. These steps are graphically depicted in Figure 1.2.

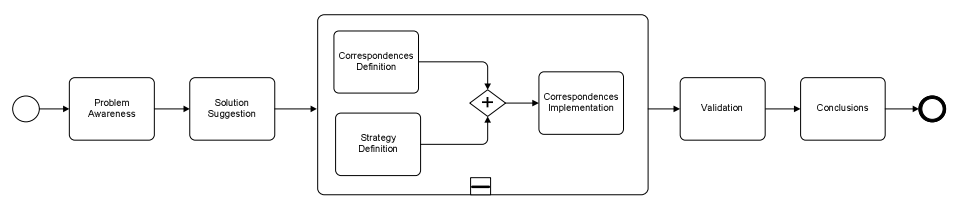

Figure 1.2 Research methodology followed in this thesis 
First of all, according to this methodology, we identified and stated the problem subject of this thesis. As a result, the boundaries of the problem being considered were clearly identified.

Then, the second step in the methodology involved suggesting a solution to the previously identified problem. The solution was designed taking into account the research developed in the Web Engineering area. However, in contrast to the solutions proposed in this area, the suggested solution was also influenced by the architecture used by Business Process Management Systems (BPMS). Therefore, not only new abstractions for describing BPdriven Web applications were identified, in addition, a revision of the architecture of the generated Web applications was performed.

During the third step, the solution designed in the previous step was developed. This development was in turn performed in several sub steps as follows:

- First, the correspondences between the identified abstractions and different software artifacts were designed. These correspondences allowed us evolving the system specification to (1) complete the specification by building different aspects of the system and (2) generating the equivalent representation but this time in terms of an implementation technology.

- In parallel to the first sub step, we defined a strategy to implement these correspondences. This decision was highly 
influenced by the use of standards and the tool support provided by each strategy.

- After the two previously sub steps, and by following the previously defined strategy, these correspondences were implemented.

As a result of the third step, a tool implementing the proposed solution was obtained. With this tool we could validate the proposed solution by developing different case studies. This validation constitutes step four.

Finally, after putting in practice the proposed solution and observing the obtained results, a set of conclusions have been presented. In addition, new research challenges emerged to this work are also proposed.

\subsection{Thesis Development Context}

This work has been developed in the OO-Method group, which belongs to the Department of Information Systems and Computation at the Technical University of Valencia. The OOMethod group research interests span multiple areas that involve Requirements Engineering, Web Engineering, Human-ComputerInteraction, Software Quality and Ambient Intelligence, all of which are tackled from the modelling perspective.

The work developed in this thesis started in the Web Engineering subgroup. This group has developed a Web Engineering method that covers the most requested requirements of current Web 
applications. The works that have enabled the development of the present thesis are framed in the following projects:

- "DESTINO: Desarrollo de E-Servicios para la nueva sociedad digital”. CICYT Project referenced as TIN200403534. (From 2004 to 2007).

- "WEE-NET: Web Engineering Network of Excellence“. E.U. ALFA (América Latina - Formación Académica) Programme.

- "SESAMO: Construcción de Servicios Software a partir de Modelos". CICYT Project referenced as TIN2007-62894. (From 2008 to 2010).

\subsection{Structure of the Thesis}

This document has been organized in five parts as follows:

\section{Part one. Introduction}

- Chapter 2. Foundations. This chapter provides the foundations for this work, these span Web Engineering, $\mathrm{BP}$, and a combination of these two areas, BP-driven Web applications. First, the chapter presents the basics of the Web engineering method that has been taken as the basis for this thesis. Second, it presents the BP notation and the executable language used in the development of this work. Finally, it states the characteristics that are observable in BP-driven Web applications. Based on these characteristics it proposes a set of requirements that Web Engineering methods should satisfy in order to specify BP-driven Web applications. 
- Chapter 3. State of the Art. This chapter provides an analysis of the literature found in the Web engineering area. In particular, this chapter focuses on the solutions proposed by each of the reviewed methods regarding the integration of BP.

\section{Part two. OOWS-BP Extension}

- Chapter 4. Development Process. This chapter provides the big picture of the proposal. It presents the set of models that are included in the proposal, the relationships among all of them, and the artefacts consumed and generated in each step of the development process.

- Chapter 5. Business Process Model. This chapter presents the Business Process Model that has been defined for the specification of BP. This model is based on the BPMN notation, we also discuss the limitations found in the original notation and extend it for use in the context of this work.

- Chapter 6. Navigational Extension. This chapter presents the navigational abstractions that have been defined to cope with the construction of the GUIs that support BPs.

- Chapter 7. Presentation Extension. This chapter presents the abstractions that have been defined in the Presentation model in order to properly specify BP-driven Web applications. In addition, based on usability requirements, this chapter covers the mechanisms provided by the proposal to obtain a more usable GUI. 


\section{Part three. OOWS-BP Framework}

- Chapter 8. Architectural Extension. This chapter presents the architecture of the generated applications to provide support for BP-driven Web applications.

- Chapter 9. Model Transformations This chapter presents the transformations that have been designed to obtain an executable web application. These transformations cover (1) the generation of executable BP and (2) the generation of the proper user interface.

- Chapter 10. Tool support. This chapter presents the tool that has been developed within the context of the Eclipse project to support the development process of $\mathrm{BP}$-driven Web applications.

\section{Part four. Conclusions and Further work}

- Chapter 11. Conclusions and Further Work. This chapter summarizes the main contributions of this work and presents future research work.

\section{Part five. Appendixes}

- Appendix A. This appendix introduces the Library4U case study that is used throughout appendixes $\mathrm{B}$ and $\mathrm{C}$ to illustrate how short-running and long-running processes are modelled following the proposed approach. Moreover, the development of these case studies allows us to validate the proposal. 
- Appendix B. This appendix extends the Library4U case study presented in appendix A to present a short-running BP.

- Appendix C. This appendix extends the Library4U case study presented in appendix A to present a long-running BP. 


\section{Chapter 2}

\section{Foundations}

This chapter presents the concepts and notions in which this thesis is based on and that are going to be used throughout this thesis. This work is related to two areas which are Web Engineering (WE) and Business Processes (BP). Within the WE area, we have applied the developed work to the OOWS approach (Fons, 2008). Therefore, in section 2.1 we present the basics of this approach. Similarly to the WE area, within the BP area there are several notations (BPML, BPMN, XPDL, UML Activity Diagrams) and executable languages (WS-BPEL, XPDL) that can be used to specify and execute processes. We have chosen the process standards BPMN (OMG, 2006) and WS-BPEL (Andrews et al., 2003), which deal with BP specification at different levels of abstraction (modelling and execution respectively). Finally, in section 2.3 we state the characteristics that differentiate the two different types of processes covered in this thesis which correspond to short-running and long-running BPs. Based on the stated characteristics, we propose a set of requirements that Web Engineering methods should satisfy in order to specify properly what we have named BP-driven Web applications. 


\subsection{Web Engineering}

To deal with the particularities that characterize Web applications compared to traditional software (desktop applications), researchers started working on the definition of methods for the systematic construction of this kind of systems. These methods were not defined from the scratch; in fact, they were based on existing methodologies for traditional software development such as object oriented techniques. The main contributions of these new methods were the introduction of new abstractions to deal with modeling navigation and presentation concerns.

In the following subsections we present the basics of the Web Engineering method in which we have applied this thesis work, the OOWS approach.

\subsubsection{The OOWS Web Engineering Method}

OOWS (acronym standing for "Object-Oriented Web Solution") is a method that was conceived to provide a methodological support for the development of Web applications applying the principles of the Web Engineering. This method defines a set of extensions to the OO-Method approach (Pastor et al., 2001) aimed at the construction of object-oriented applications. In particular, this extension introduces the User, Navigational and Presentation Models which allows describing the requirements that define the interaction of the user with those systems. The extension also defines new model compilers that allow completing the code 
generation phase by transforming these new models into their corresponding software artefacts.

\subsubsection{The OOWS User Model}

The User Model allows identifying and categorizing users. It allows determining the kind of users that are going to interact with the system. These kinds are organized within a hierarchical structure that represents the specialization/generalization relationships between them. In addition, this model allows characterizing users into three groups which are:

- Anonymous. This kind of users does not need to be identified in the system to access to it. Normally, they have a very limited access to data and functionality. In particular "sensitive", "critical" or "personal" data is hidden to anonymous users.

- Registered. This kind of users requires to be identified in the system and represents users with access to data and functionality defined as "sensitive", "critical" or "personal".

- Abstract (or without access). This kind refers to "virtual" types of users which are defined (1) to express the set of common responsibilities defined for a group of users, (2) to capture the functional hierarchy of the organization and (3) to organize and structure the users of the system.

The graphical notation used to identify types of users according to their access permission is presented in Figure 2.1. 

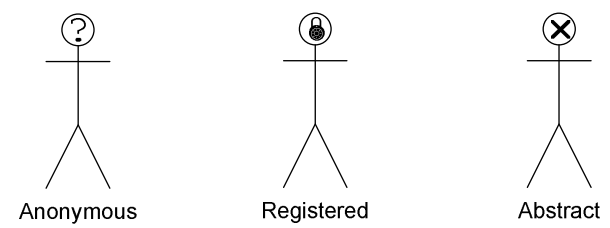

Figure 2.1 Types of Users According to the Different Access Permission

Regarding the relationship between user types, this mechanism allows inheriting navigational properties between them. In addition, child user types can modify the inherited navigational maps by adding or removing access to nodes or links. Figure 2.2 shows the User Model defined for the case study developed in Appendix A. In it, the Librarian and Secretary types inherit the navigational properties defined for the Member type which in turn inherits from the Anonymous user type.

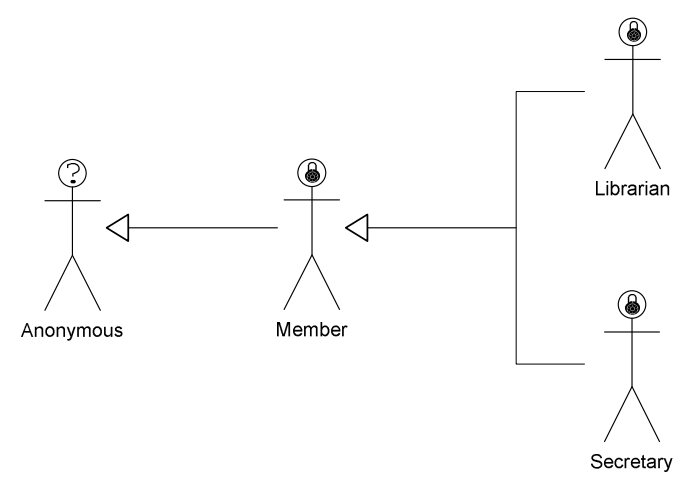

Figure 2.2 Example of User Specialization

\subsubsection{The OOWS Navigational Model}

The Navigational model has been defined to describe the system accessibility for the type of users defined in the User Model. This model is built in two steps. First of all a global description of the interaction points with users is performed. This step is called the "Authoring-in-the-large" step. Then, a detailed description of these 
interaction points is performed. This step is called "Authoring-inthe-small" step.

\section{Authoring-in-the-large Step}

During this step, we describe the way a particular type of user will be able to navigate through the system for consulting some information and performing some functionality, attending to her responsibilities and privileges as user of the system.

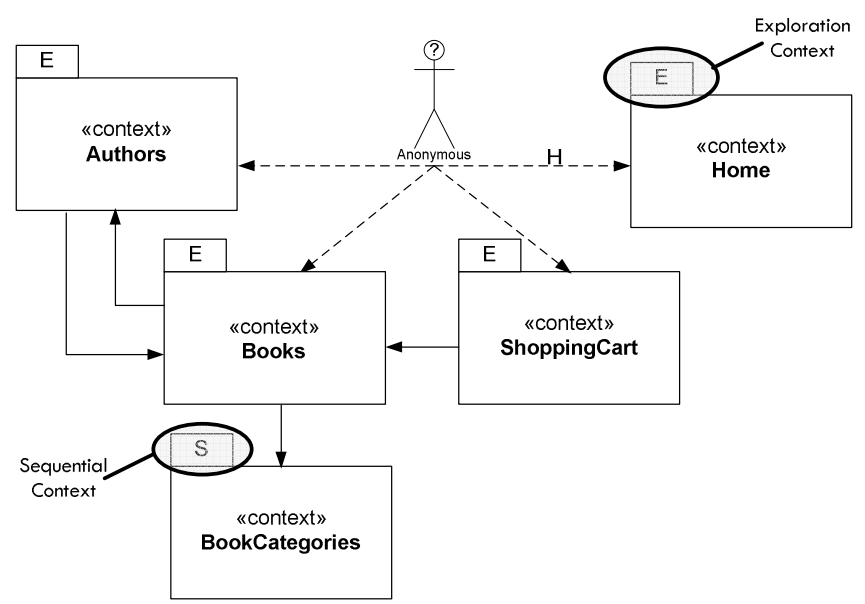

Figure 2.3 Navigational Map for the Library4U Case Study

Figure 2.3 represents the Navigational map built for the Library $4 \mathrm{U}$ case study. This map is represented as a directed graph whose nodes represent navigational contexts or navigational subsystems and whose arcs represent navigational links that define the valid navigational paths over the system. Navigational links correspond to arcs from the navigational map and are used to define reachability paths among different nodes. There are two types of navigational links: 
- Sequence links or "contextual links" (represented using solid arrows) involve a semantic navigation between two contexts understanding semantic navigation as the activity of carrying some information from a source context to target context.

- Exploration links or "non contextual links" (represented using dashed arrows) represent valid navigation paths through different contexts. In contrast to the navigation defined by sequence links, this navigation does not involve carrying information between contexts. These links are implicitly defined by exploration contexts or exploration subsystems.

During this stage, without specifying the details of the nodes, we can build a system accessibility structure that allows organizing the tasks and responsibilities of a particular kind of user.

\section{Authoring-in-the-small Step}

Within this step, the navigational contexts (nodes) defined in the navigational map built previously are detailed (see Figure 2.4). Navigational contexts are made up of a set of Abstract Information Units (AIU), which represent the requirement of retrieving a chunk of related information. AIUs are made up of navigational classes, which represent views over the classes defined in the Class Diagram. These views are represented graphically as UML classes that are stereotyped with the "view» keyword and that contain the set of attributes and operations that will be available to the user. Each AIU must include one navigational class (called 
the manager class) and can optionally include a set of complementary class views (called complementary classes) to complete the information retrieved by the manager class.

Navigational classes are related by unidirectional binary relationships called navigational relationships. These relationships are defined over existing association or inheritance relationships defined in the Class Diagram. Moreover, depending on the navigational capability of the navigational relationship these can be of two types:

1. Context Dependency Relationship (graphically represented using dashed arrows) which represents a basic information retrieval by crossing a structural relationship between classes. When a Context Dependency Relationship is defined, all the object instances related to the origin class object are retrieved.

2. Context Relationship (graphically represented using solid arrows) which represents the same information recovery as a Context Dependency Relationship does plus a navigation capability to a target navigational context, creating a sequence link in the navigational map. 


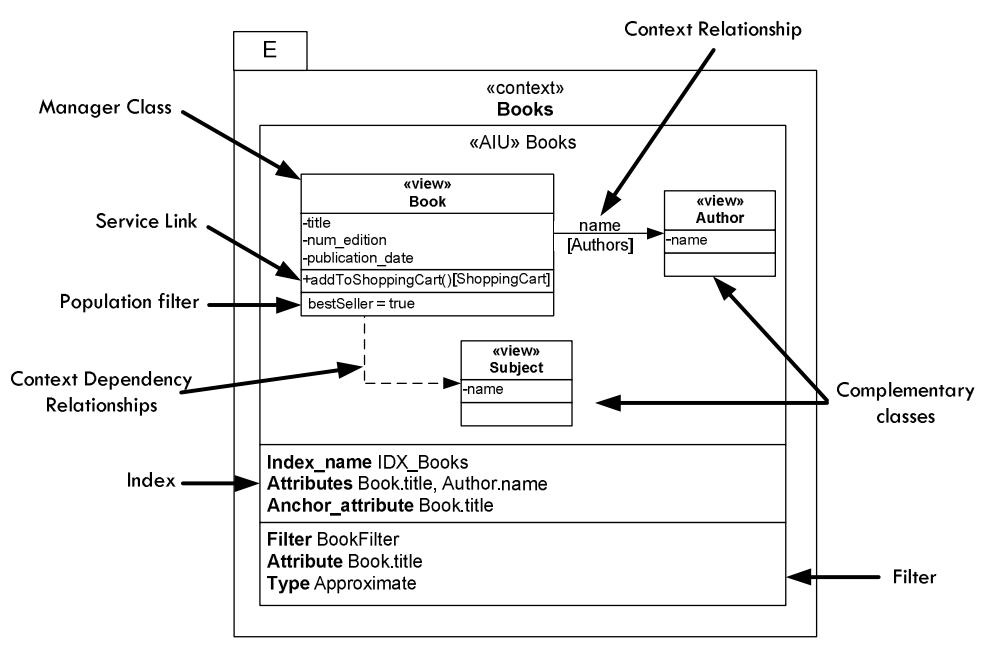

Figure 2.4 Navigational Context

A Service Link defines a navigation that will automatically be performed after the execution of an operation defined within a navigational class (navigational operation). Figure 2.4 shows an example of a Service Link defined associated to the Book class view. This Service Link defines the navigation to the target context (ShoppingCart) that will be performed each time the "addToShoppingCart()" is executed inside this context.

Apart from attributes and operations, navigational classes can also define conditions to filter the retrieved objects. These filters are called Population Filters and are specified by means of OCL formulas at the bottom section of the class view primitive. In Figure 2.4, a population filter over the Book class view has been defined to indicate the retrieval of just those books whose attribute "bestSeller" is set to true. 
These are the core primitives for navigational specifications. However, this specification can be enriched by introducing mechanisms to help the user explore and filter the huge amount of information inside a context. These advanced concepts are indexes and filters. On the one hand, an index is a structure that provides an indexed access to the population retrieved by the manager class. Indexes create a list of summarized information by using an attribute or a set of attributes. When an index gets activated, a list of all the possible values for the indexed attribute/s is created. By choosing one of these values, all objects that have the same value for this property will be shown in a search view. This search view describes the information that will be available to the user to aid him/her to select an instance. This selected instance will be activated in the navigational context. On the other hand, a filter defines a population condition to restrict the object instances to be retrieved. There are three types of filters which are:

- Exact filters, which take one attribute value and return all the instances that match it exactly.

- Approximate filters, which take one attribute value and return all the instances whose attribute values include this value as a substring.

- Range filters, which take two values (a maximum and a minimum) and return all the instances whose attribute values fit within the range. If we specify only one value, it is only bounded on that side (upper or lower bounded). 


\subsubsection{The OOWS Presentation Model}

The Presentation Model allows describing presentation requirements by means of a set of basic patterns. This model is strongly dependent on the navigational model since it uses navigational contexts (system-user interaction units) to define the presentation properties. Presentation requirements are specified by means of patterns that are associated to the primitives of the navigational context (navigational classes, navigational links, searching mechanisms, etc.). The basic presentation patterns are:

- Information Paging. This pattern allows us to specify information "scrolling". All the retrieved instances are broken down into logical blocks so that only one block is visible at a time. Mechanisms to move forward or backward are provided. The required information is:

- Cardinality, which represents the number of instances that make a block.

- Access mode, which can be defined as Sequential, providing mechanisms to go to the next, previous, first and last logical block or Random, where the user can directly access any block.

- Circularity. When this property is active, the set of blocks behaves as a circular buffer.

- Ordering Criteria. This pattern defines a class population ordering (ASCendant or DESCendant) using the value of one or more attributes. It can be applied either to navigational classes or access structures, specifying how the retrieved instances will be ordered. 
- Information Layout. We provide three basic layout patterns and one layout operator. The three patterns are: register, tabular (vertical and horizontal), and tree. The operator pattern (master-detail) is applied to many-to-many relationships using one of these basic layout patterns to show the detail portion.

These presentation patterns, together with the specified navigation features, capture the essential requirements for the construction of web interfaces.

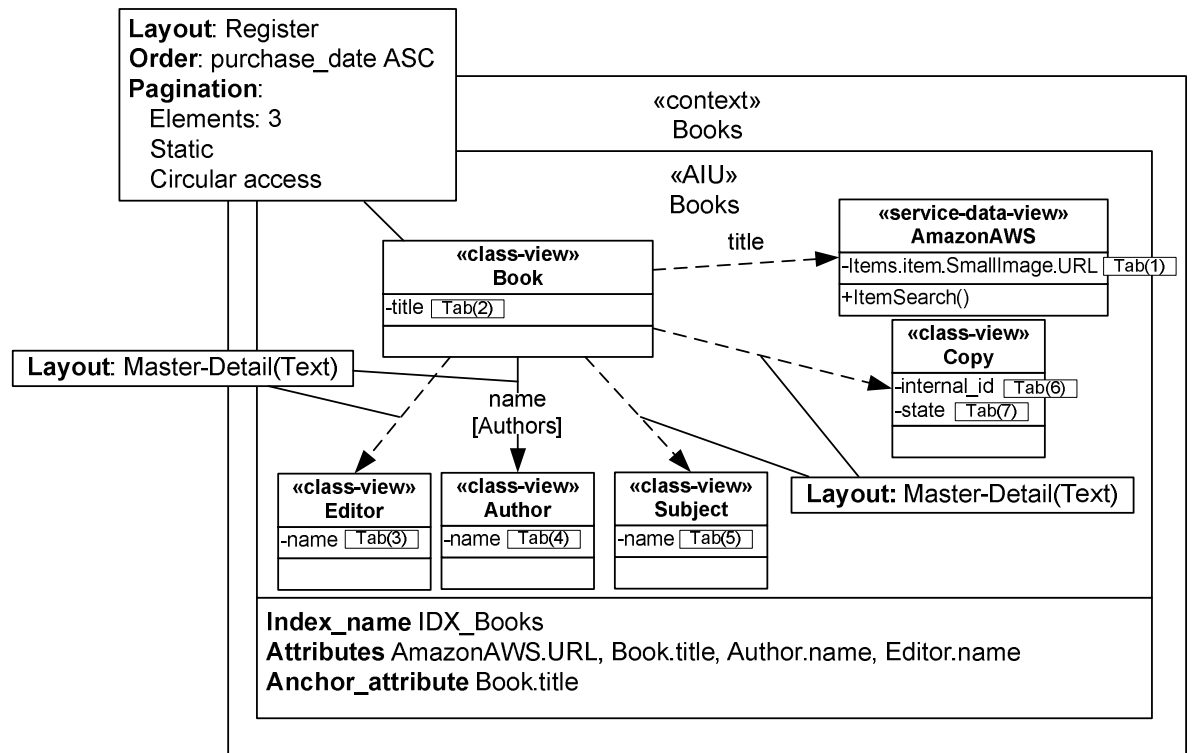

Figure 2.5 Layout Patterns applied to Navigational Class and Relationships

Figure 2.5 shows the presentation attributes associated to the Book navigational context depicted in Figure 2.4. In particular, the Register and Master-Detail(Text) Information layout patternS have been associated to the classes included in the AIU. 
In Figure 2.5, the application of the Information layout pattern to the manager class and the navigational relationships is presented. On the one hand, the register pattern is only applied to the manager class. On the other hand, since a Book can have related multiple authors, editors, subjects and copies, the tabular pattern cannot be applied. In this case the master-detail and the text layout pattern are combined. The master-detail pattern creates a link between the information from the manager class (class playing the role of master) and the complementary classes (detail). The text layout pattern specifies that data is retrieved without any format.

In this section we have presented the basics of the OOWS approach which are necessary for the explanation of the presented thesis. However, a complete description of this method is presented in (Fons, 2008).

\subsection{Business Processes}

The objective of this section is to present the specifications that are going to be used along this work to deal with the representation of BPs. Attending to the two different levels of abstractions defined in the proposed method (modelling and implementation) the following two subsections present respectively the BPMN notation (graphical notation used to represent BPs at the modelling level) and the WS-BPEL language (specification used to execute the BPs represented graphically at 
the modelling level). This differentiation is necessary since WSBPEL, although being a powerful language, it is difficult to use and it is not very intuitive to end-users.

\subsubsection{BPMN: Business Process Modelling Notation}

The BPMN standard was developed by the BPMI (Business Process Management Initiative) mainly to provide a notation that could be easily understood by all business stakeholders. In addition to this goal, it was also developed to ensure that XML languages designed for the execution of BP (such as WS-BPEL) could be visualized with a business-oriented notation. As a result, this notation constitutes a standardized bridge between the BP definition and its implementation.

The common characteristics that are observable in $\mathrm{BP}$ design notations are:

- Capability for modeling the sequence of activities.

- Capability for modeling the data or messages interchanged between the participants involved in the BP definition.

- Capability for modeling different participants and different roles within these participants.

- Capability of understanding their internal business procedures in a graphical notation and will give organizations the ability to communicate these procedures in a standard manner. 
Specifically, the BPMN notation ${ }^{10}$ provides a set of elements to deal with the characteristics presented above. This set of elements is organized in four categories which are Flow Objects, Connecting Objects, Swimlanes and Artifacts:

\section{Flow Objects}

These elements constitute the main graphical elements to define the behaviour of a Business Process. These refer to:

- Events. An event is something that "happens" during the course of a business process. These events affect the flow of the process and usually have a cause (trigger) or an impact (result). Events are circles with open centre to allow internal markers to differentiate different triggers or results. There are three types of events, based on when they affect the flow: Start, Intermediate, and End. Figure 2.6 shows the complete set of events defined by the notation.

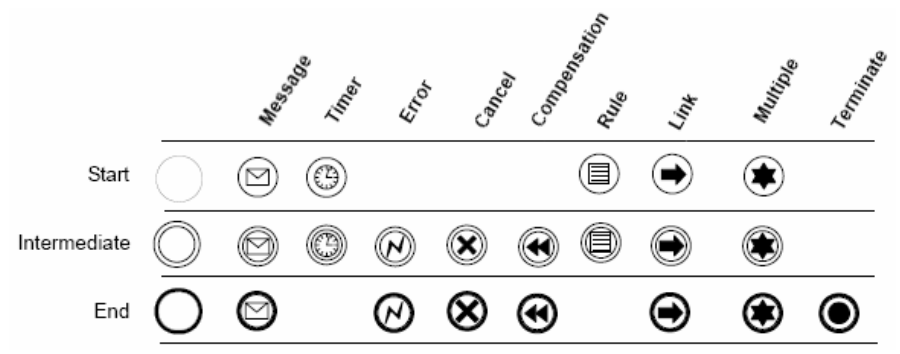

Figure 2.6 Events Graphical Elements

- Activities. An activity is a generic term for work performed within a BP. An activity can be atomic or non-atomic

10 The BPMN version used in this work corresponds to the version 1.1 
(compound). The types of activities that are a part of a Business Process Diagram are: Process, Sub-Process, and Task. Only Tasks and Sub-Processes define a specific graphical object (a rounded rectangle). On the contrary, Processes are built as a set of activities and the controls that sequence them. In addition, Tasks and Sub-Processes include a set of attributes which determine if these activities are repeated or performed just once. This repetition can be performed either sequentially (loop marker) or in parallel (parallel marker). Figure 2.7 depicts the different types of activities and the markers available to specify when the activity can be repeated and how.

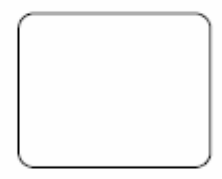

Task (Atomic)

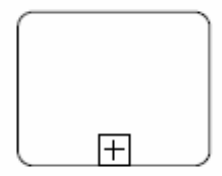

Process/Sub-Process Collapsed

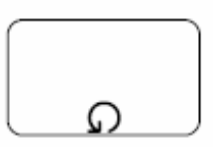

Activity Looping

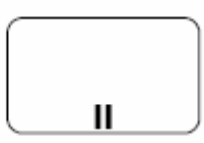

Multiple Instances

Figure 2.7 Activities Graphical Elements

- Gateways. A Gateway is used to control the divergence and convergence of Sequence Flows. Thus, it will determine branching, forking, merging, and joining of paths. Internal markers will indicate the type of behaviour control. Figure 2.8 depicts the different types of gateways provided by the notation. 


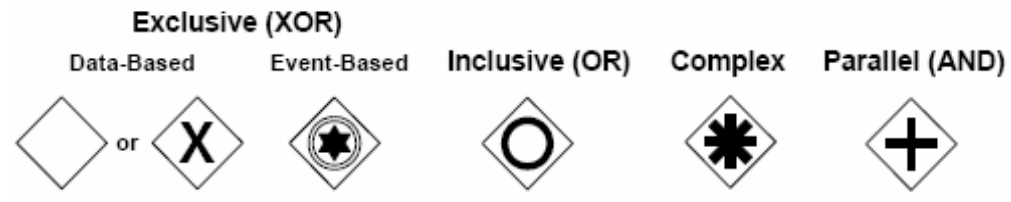

Figure 2.8 Gateways Graphical Elements

\section{Connecting Objects}

This element allows connecting Flow objects or other information. The connecting objects defined by the notation are:

- Sequence Flow: It is used to show the order in which activities will be performed in a Process. This type of connecting object can in turn be specialized in Normal, Conditional and Default Flow.

- Message Flow: It is used to show the flow of messages between two participants that are prepared to send and receive between them. In BPMN, two separate Pools in the Diagram will represent the two participants (e.g., business entities or business roles).

- Association: An Association is used to associate information with Flow Objects. Text and graphical non-Flow Objects (i.e. data objects) can be associated with Flow Objects.

Figure 2.9 depicts the different connecting objects defined by the BPMN notation. 


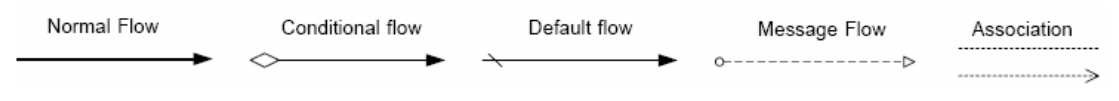

Figure 2.9 Connecting Objects Graphical Elements

\section{Swimlanes}

This element allows grouping Flow objects based on a particular criterion. This category includes two types of elements which are:

- Pools: represent a Participant in a Process.

- Lanes: are used to organize and categorize activities. This is achieved by partitioning the Pool in different lanes.

Figure 2.10 depicts the different types of elements defined by the Swimlane group.
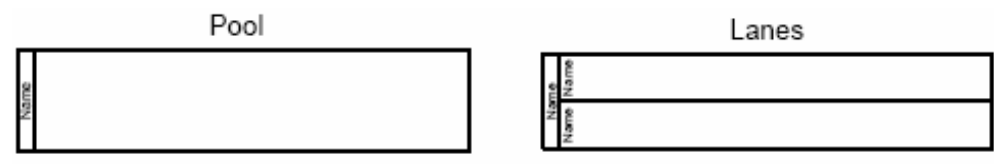

Figure 2.10 Swimlanes Graphical Elements

\section{Artifacts}

The elements included within this type are introduced into BP models to improve their understanding. Within this category we find:

- Data Object: This element provides information about activity requirements and results.

- Group: The grouping can be used for documentation or analysis purposes. Groups can also be used to identify the activities of a distributed transaction that is shown across Pools. 
- Annotation: Text Annotations are the mechanism provided to modellers to introduce additional information for the reader of a BPMN Diagram.

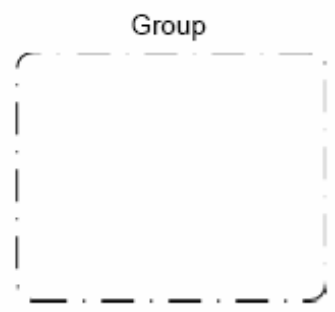

Data Object

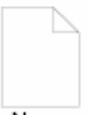

Name
Text Annotation

Descriptive Text Here

Figure 2.11 Artifacts Graphical Elements

In addition to these four categories, the BPMN notation handles advanced modelling concepts such as:

- Exception handling. By means of this mechanism we can capture and handle exceptions during the performance of the process. The way to capture them is by the use of Intermediate Events (Message, Timer, and Rule) which act like a forced end to an activity and Exception Flows.

- Transactions. These are used during the definition of SubProcesses. Associated to transactions we can define the protocol that is going to be used to control the behaviour of the Sub-process as well as the technique that will be used to undo a transaction when this has been cancelled.

- Compensation. When the outcome of some activities result in an undesirable situation, compensation can be specified to undo the obtained results. To represent this scenario the BPMN notation provides the Compensation Intermediate Event, Association and Activity. 
The elements presented above correspond to the version 1.0 of the notation (OMG, 2006). This version was adopted by the OMG on February 2006 as the official specification. However, a RFP for the 2.0 version has already been released (OMG, 2007b). The objective of this RFP is to define a complete and consistent language based on the BPMN and the Business Process Definition Metamodel (BPDM) (OMG, 2007a) that unifies their capabilities (graphical notation, metamodel and interchange format).

BPMN was not been conceived to model applications; instead, it was conceived to model processes which will be executed within these applications. Therefore, a BPMN model needs to be expressed in a language that could be interpreted and executed by the software in charge of executing them. In this context, languages such as BPML, WS-BPEL and XPDL come into play. From this set of languages we have opted for the standard which is the WS-BPEL language and is presented later in the following subsection.

\subsubsection{WS-BPEL: Business Process Executable Language for Web Services}

Business Process Executable Language for Web Services (WSBPEL) is a XML-based language that allows orchestrating interactions between Web services in a standardized manner. This language is based on Web services, in fact, each business process is assumed to be implemented as a Web service. Basically, a WS- 
BPEL process describes a flow of interactions that occur between the process and a set of services. The basic elements of a WSBPEL document are activities (units of work carried out to complete the process) and partners (elements responsible of performing process activities). Figure 2.1 depicts this basic elements and how this relate to each other. Moreover, it also depicts the elements defined in a WSDL document and the mappings defined between these elements and the basic elements of a WS-BPEL document.

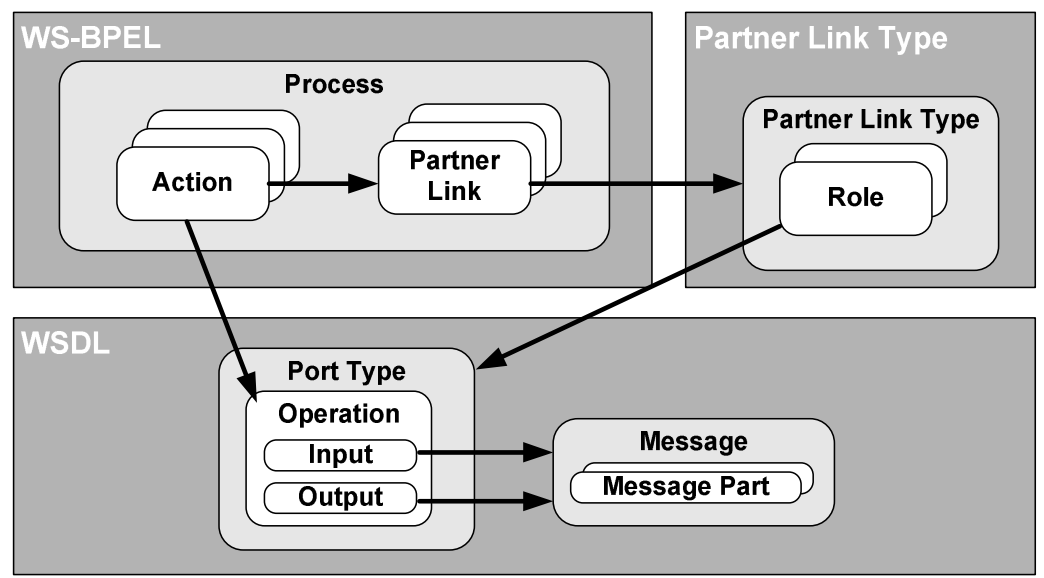

Figure 2.12 Relation between WS-BPEL Process Definition and WSDL

Moreover, other elements such as correlation, data compensation, fault and events handlers are also defined by the language. The set of elements defined by the WS-BPEL language are:

\section{Partner Link Types}

This element is used to characterize a conversation between two services. In this conversation the roles played by each service is specified as well as the port type provided by the service to receive messages. Figure 2.13 depicts graphically the structure of this 
element. A Partner Link Type can define one or two roles. One role is defined for One-way and Notification operations. On the contrary, two roles are defined for Request-response and Solicitresponse operations. The only requirement when two roles are defined is that the calling service will receive a response (callback) from the requested service along the conversation.

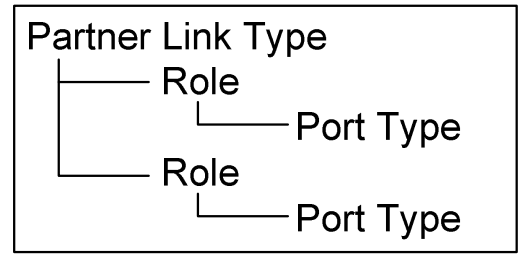

Figure 2.13 Partner Link Type structure

\section{Partner Links}

This element represent each of the partners involved in the process (the own WS-BPEL process is also considered one of the partners). It is necessary to specify for each conversation the role played by each of the partners involved in it which corresponds to the roles defined by the Partner Link Types as Figure 2.14 shows.

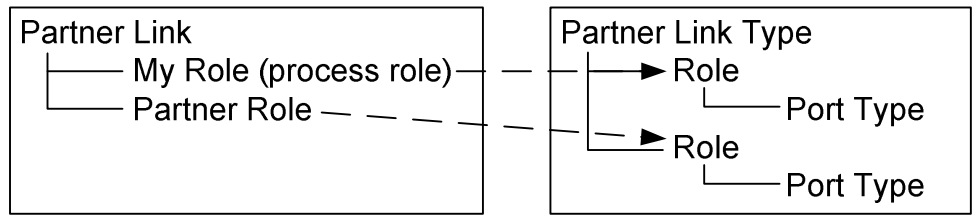

Figure 2.14 Partner Link structure

More than one Partner link can be characterized by the same Partner Link Type allowing the use of multiple vendors within the same process.

\section{Variables}


Variables are used to handle data (receive, manipulate and send data during partner conversations) and allow maintaining the state of the process. The types of these variables relate to data types defined externally to the WS-BPEL process.

\section{Activities}

Activity elements describe the steps that make up a process (the order in which these occur is defined by the flow of the process). In WS-BPEL we can define three different types of activities which are:

- Basic. This type of activities are used to represent invokes or receptions on service operations. These types are "invoke", "receive" and "reply".

- Structured. This type of activities is used to specify the order in which a set of activities can take place. Within this group we find ordinary sequential control by the use of "sequence", "switch" and "while" types, concurrency and synchronization between activities by the use of the "flow" type and nondeterministic choice based on external events by the use of the "pick" type.

\section{Correlation}

This is the mechanism provided by WS-BPEL to allow delivering messages to the proper process instance. WS-BPEL addresses correlation scenarios by providing a declarative mechanism to specify correlated groups of operations within a service instance. A set of correlation tokens is defined as a set of properties shared by all messages in the correlated group. 
All these elements are defined in the WS-BPEL version 1.1. This version constitutes an enhanced version of the BPEL 1.0 version and was submitted to OASIS in April 2003. As we can observe from the elements presented above, version 1.1 of the WS-BPEL language does not provide support for human interactions. In fact, it is necessary to incorporate new elements to the original specification to deal with this issue. However, the incorporation of new elements difficult the portability of the WS-BPEL document because this usually ties the document to the proprietary implementation of the provider.

Related to this limitation, researchers from Active Endpoints, Adobe, BEA, IBM, Oracle and SAP have defined two specifications which introduce a comprehensive human interaction model for WS-BPEL. These extensions are:

- BPEL4People (Agrawal et al., 2007) which provides an extension to BPEL to address human interactions as a first-class BPEL citizen. It defines a new type of basic activity which uses human tasks as an implementation, and allows specifying tasks local to a process or use tasks defined outside of the process definition. This extension is based on the WS-HumanTask specification.

- WS-HumanTask (Agrawal et al., 2007a) provides the definition of human tasks, including their properties, behaviour and a set of operations used to manipulate human interactions. A coordination protocol is introduced 
to control autonomy and life cycle of service-enabled human tasks in an interoperable manner.

In this thesis, since these two specifications did not exist, we defined a Task Manager Service (see chapter 8) in charge of handling the interaction with human participants.

\subsection{Business Process-driven Web Applications}

A Business Process-driven Web application is defined as an application that is accessed via a Web browser over a network (i.e., the Internet or an intranet) and that provides support for the execution of BP. The type of BPs that can be supported by these applications includes different degrees of complexity, where the complexity is given by the number and the type of interactions defined by the BP. Based on this complexity we have catalogued BPs into two types which are named short-running and longrunning BPs. These types are explained in detail in the following subsections. However, before we are going to present the common characteristics that are required in BP-driven Web applications, independently of the type of BP supported.

- Short\&Long 1. Users need mechanisms for launching BP instances and taking up again BPs that have not been completed.

- Short\&Long 2. Users need mechanisms for completing BP activities. These mechanisms have to be provided according to the type of activity been performed. For instance, when the activity requires the introduction of data, the user has 
to be provided with an interface that allows her inputting this data.

- Short\&Long 3. Users must be guided through the steps that allow achieving the $\mathrm{BP}$ goal.

- Short\&Long 4. Users can leave (suspend) the execution of a BP for a while and taking up again (resume) the BP later on. Therefore, the system has to keep the state of the process to restore the $\mathrm{BP}$ when this is taken up again.

- Short\&Long 5. Some of the activities defined in a BP can be performed by external system.

\subsubsection{Short-running Business Processes}

This type of processes is also called in the literature "light-weight processes". As the following list of requirements shows, its effect has a short period of time and its consequences of a wrong decision are small. Examples of this kind of processes are the "Check out process" usually found in online stores or the "Booking service" usually found in online travel agencies. These types of processes are characterized by the following features:

- Short 1: Are completed in a very short period of time (intervals can range from seconds to a few hours).

- Short 2: Involve just one human participant (who interacts with the system/process to accomplish it) and one or more "automated" participants.

- Short 3: Are usually simple (in terms of control flow) and not very large (big).

- Short 4: Are always started by the user (human participant). 
- Short 5: The user only participates in one case (instance) of the process at the same time.

\section{Example: Checkout Business Process}

In order to exemplify this type of BP we present in Figure 2.15 the Checkout BP. This process involves the participation of two agents. One of them refers to a human being (lane labelled as "Member") and another to an automated system (lane labelled as "System"). The process details the steps that are required to accomplish the virtual purchase.

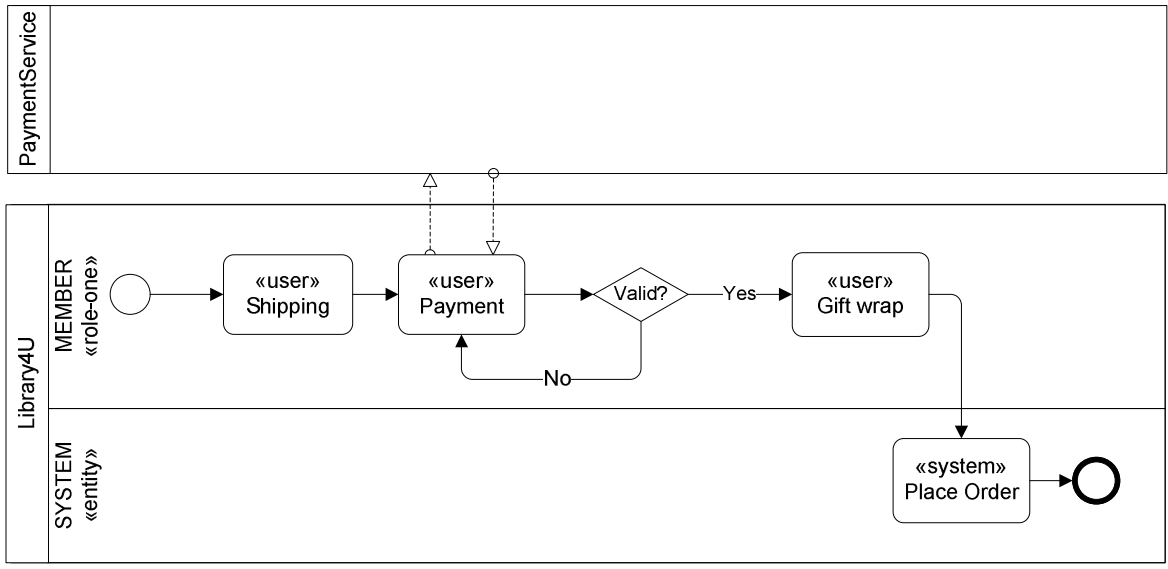

Figure 2.15 Example of a short-running process: Checkout Process

In this $\mathrm{BP}$, it is the human participant (Member role) who starts the checkout process. The first task in the process asks the user to input information about the shipping details (address, city, country, shipping mode, etc). In the second task the user is asked to input information about payment options (credit card, PayPal account, security codes, etc). Once all of this information is inputted, the process starts a payment validation step by invoking an external validation service provided by a payment service. If 
the external service validates the inputted data then the user is asked about wrapping options. Otherwise, the user is redirected to the payment step to introduce again the payment data. Finally, the process concludes by creating and placing the order in the system.

Taking up again the characteristics that are observable in shortrunning BP we can see that all of them are satisfied in the Check out BP example:

- Short 1: The completion of the BP only depends on the Member user (we assume that automated activities are completed instantaneity, i.e., the "Place order" activity included in the System lane). Therefore, if the user does not suspend the $\mathrm{BP}$, this can be completed in a few minutes.

- Short 2: The BP just includes one human participant (represented by the Member lane) and one system (represented by the System lane).

- Short 3: The BP is quite simple and not too long, it only includes four activities.

- Short 4: The Member user (human participant) is the one launching an instance of the BP.

- Short 5: The Member user cannot participate in more than one instances of the Checkout BP at the same time.

Figure 2.16 shows the Web interface that is provided to the Member user to complete one of the activities defined in the Check out BP. Specifically it shows the interface provided for accomplishing the Shipping task where the user has to introduce 
all the information required by the underlying operation associated to this task (address, city, country, etc.)

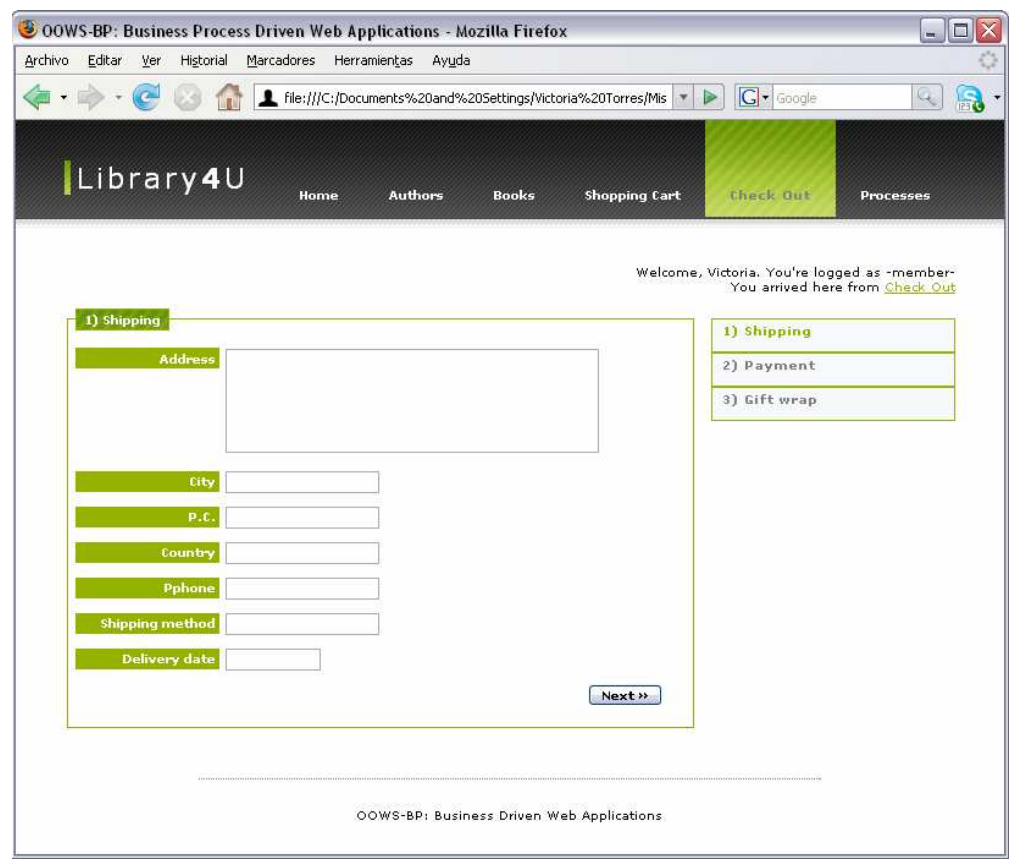

Figure 2.16 Web interface to complete the first activity of the Checkout BP

\subsubsection{Long-running Business Processes}

These processes usually define the protocols that have to be followed within an organization to achieve a specific goal. Examples of this kind of processes are the "Material Purchase Requests" or the "Incidence Management Requests". In general, this kind of processes involves not only the coordination of different systems but also the coordination of different people behaving with different roles. The following list gathers the features/requirements observed in this kind of processes:

- Long 1: Usually take a long time for being completed. There are several reasons that justify this prolongation in 
time such as several human participants, temporal constraints, etc.

- Long 2: Usually involves more than one human participant and one or more automated systems.

- Long 3: Can include temporal constraints that provoke that some tasks cannot be started until a specific instant of time.

- Long 4: Can be started by any participant involved in the process. (Therefore, it is advisable that users could find easily the set of pending tasks that she/he is responsible of).

- Long 5: The user can participate in more than one case (instance) of the process at the same time.

\section{Example: Book Purchase Request Business Process}

To exemplify this kind of processes we are going to make use of the "Book Purchase Request" BP depicted in Figure 2.17.

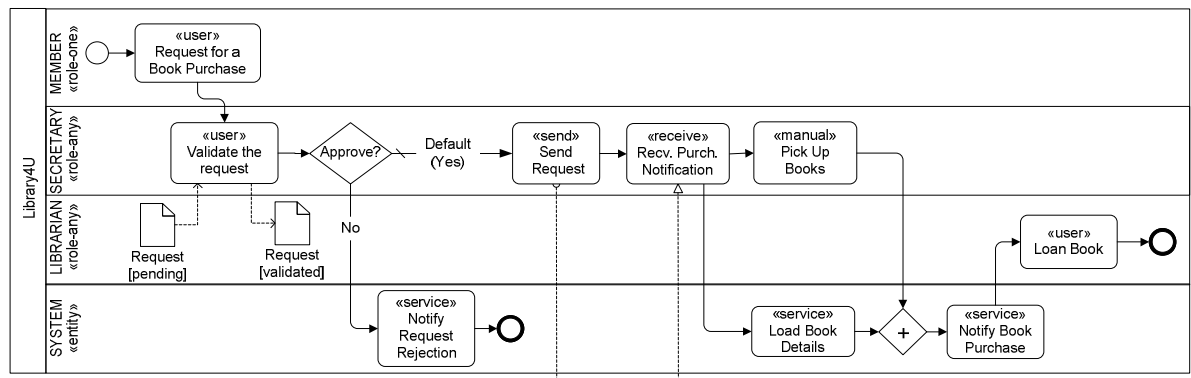

Figure 2.17 Example of a long-running process: Book Purchase Request

This BP is started by a Member user who wants a specific book that it is not catalogued in the library. Therefore, the user 
provides in the first step the information of the specific book (i.e., book title or ISBN). Then, the book request is processed by any user belonging to the Secretary User type. In this step, the user validates the request based on some criteria. If the Secretary User denies the purchase request, the system notifies the member (usually by sending an e-mail). On the contrary, if the purchase request is approved, this request is redirected to the Central Library, which is responsible of the purchase of the requested books. At this point the process is waiting for the purchase notification response sent back by the Central Library. When this notification arrives, the system must load the book details into the Library $4 \mathrm{U}$ system and in parallel, any user belonging to the Secretary group has to pick up (manual operation) the book from the Central Library. When these two tasks are completed, the system notifies the member about the acquisition of the book and finally one of the users belonging to the Librarian User type finalizes the process by realizing the book loan to the solicitor member.

Again, the characteristics found in this example map to the ones that are observable in a long-running BP:

- Long 1: In this case, the existence of several human participants propitiates the prolongation in time of the BP.

- Long 2: The BP involves three human participants which refer to users belonging to the Member, Secretary and Librarian User types. In addition it also includes an automated system which refers to the local system. 
- Long 3: In this case the temporal constrains are introduced by the time that different human participants take to complete their assigned activities.

- Long 4: In this case the BP is started by a human participant. However, compared to the Checkout BP, new human participants are involved in the BP.

- Long 5: Any of the human participants defined in the BP can participate in different instances of the same BP. For instance, a Secretary user has to validate each book request launched by any member.

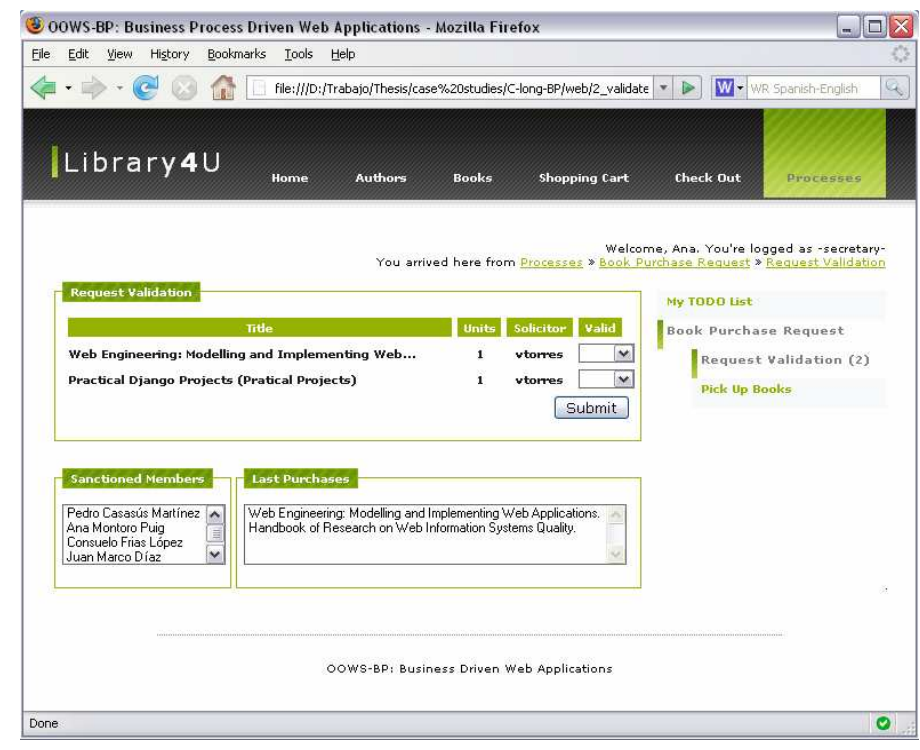

Figure 2.18 Web interface to complete the "Request Validation" activity of the long-running BP

Figure 2.18 shows the Web interface that is provided to the Secretary user to complete the "request validation" task. In this case, we can appreciate that there are two different instances of this task. 


\subsubsection{Requirements for Dealing with BP-driven Web applications}

Based on the characteristics that are observable in both types of BPs, we enunciate the requirements that Web Engineering methods should satisfy in order to specify and built BP-driven Web applications. Some of these requirements have already been stated by Damiano et al. in (Distante et al., 2007a).

- Requirement 1. Differentiate between Short-running and Long-running Processes. WE methods should follow a code generation strategy that allows generating appropriate GUI depending on the type of process being executed. For instance, long-running processes are better handled from a Web interface similar to the ones provided by Business Process Management solutions. This type of Web interfaces allows users to launch new process instances and complete their pending tasks. On the contrary, short-running processes are better handled from a Web interface designed following the wizard pattern.

- Requirement 2. Specify the data and functionality to support $B P$ activities. WE methods should provide mechanisms that allow specifying which data and functionality has to be provided in order to complete a BP activity.

- Requirement 3. Deal with the Specification of BP flows. WE methods should provide mechanisms that allow describing the different paths that BP can take during the execution.

- Requirement 4. Deal with Distributed Processes. WE methods should provide mechanisms that allow specifying the different 
systems involved in the process and also how these interact to accomplish the BP goal.

- Requirement 5. Deal with Multiple roles. Within a single organization, process activities are distributed among different responsibilities. Therefore, it is necessary to provide mechanisms for specifying the different roles involved in a BP and how these cooperate to achieve a common goal.

- Requirement 6. Deal with Manual Tasks. Manual tasks are commonly found in processes involving human participation. Thus, it is necessary to provide mechanisms that allow specifying tasks of this kind.

- Requirement 7. Separation of Concerns related to Navigation. In terms of model reusability and maintenance, it is very important to follow a strategy that allows a proper separation of concerns regarding navigation. This separation should clearly distinguish between the navigation that occurs during the execution of a $\mathrm{BP}$ and the navigation that occurs during content navigation.

- Requirement 8. Keep the State of the BP. To ensure that users can suspend safely the current executed BP and resuming it correctly later, it is necessary to keep the state of the launched $\mathrm{BP}$ instances.

Table 2-1 summarizes the BP characteristics that are covered by each of the requirements stated previously. 


\begin{tabular}{|c|c|c|c|c|c|c|c|c|c|c|c|c|c|c|c|}
\cline { 2 - 13 } \multicolumn{1}{c|}{} & \multicolumn{1}{c|}{ Short \& Long } & \multicolumn{9}{c|}{ Short } & \multicolumn{5}{c|}{ Long } \\
\hline & 1 & 2 & 3 & 4 & 5 & 1 & 2 & 3 & 4 & 5 & 1 & 2 & 3 & 4 & 5 \\
\hline Req1 & $\checkmark$ & & & & & & & & $\checkmark$ & $\checkmark$ & & & & $\checkmark$ & $\checkmark$ \\
\hline Req2 & & $\checkmark$ & & & & & & & & & & & & & \\
\hline Req3 & & & $\checkmark$ & & & & & & & & & & & & \\
\hline Req4 & & & & & $\checkmark$ & & & & & & & & & & \\
\hline Req5 & & $\checkmark$ & & & & & $\checkmark$ & & & & & $\checkmark$ & & & \\
\hline Req6 & & $\checkmark$ & & & & & & & & & & & & & \\
\hline Req7 & & $\checkmark$ & & & & & & & & & & & & & \\
\hline Req8 & & & & $\checkmark$ & & $\checkmark$ & & & & & $\checkmark$ & & & & \\
\hline
\end{tabular}

Table 2-1 Mapping between BP characteristics and the Requirements that should be satisfied by WE Methods

\subsection{Conclusions}

In this chapter we have presented the foundations for this thesis which span Web Engineering, BPs and Web applications that support BP execution. First, we have provided a brief description of the OOWS method. Specifically, we have focused on the Navigational and Presentation models which capture the particular requirements that introduce the specification of Web applications. In this thesis we have extended these models to capture properly the requirements of BP-driven Web applications (see chapters 6 and 7). Second, we have presented the BPMN and the WS-BPEL standards. These have been adopted in the current proposal to specify BPs at two different levels of abstraction. On the one hand, the BPMN notation allows us representing BPs at the modelling level. On the other hand, the WS-BPEL language allows us executing BPs specified at the modelling level. Specifically, the BPMN notation has been extended to integrate 
BP specifications within the method and to allow an automatic generation of BPs represented in WS-BPEL (see chapter 5). Finally, we have presented the kind of BPs that we are considering in this thesis. By means of two examples we have stated the characteristics of each type. These characteristics have been used to define a set of requirements that Web Engineering methods should satisfy in order to specify properly BP-driven Web applications. 



\section{Chapter 3}

\section{State of the Art}

This chapter provides an analysis of the most well-known methods developed within the Web Engineering field. Specifically, this analysis is focused on the solution provided by these methods to deal with the integration of BPs into Web applications. First of all, it presents the concrete solution designed by each method. This presentation includes the mechanisms provided at the modelling level, the adaptation of the development process to this new feature and, when applicable, the support provided by a tool. Then, it provides a discussion of the adequacy of the proposed solutions to deal with the construction of BP-driven Web applications. This discussion is based on the requirements that Web engineering methods should satisfy to specify BP-driven Web applications.

\subsection{Web Engineering Area}

In order to deal with the systematization of the Web application development (Pressman et al., 1998), during the late nineties, a new Engineering discipline emerged, it was called Web Engineering (WE). This term was coined in (Murugesan et al., 
1999) and was defined as "an engineering that uses scientific, engineering; and management principles and systematic approaches to successfully develop, deploy, and maintain highquality Web systems and applications". Later, different authors (Ginige et al., 2001a; Kappel et al., 2006; Mendes, 2006) have supported the necessity of this new engineering to deal with the particularities introduced by Web-based systems. As a result of the studies developed in this area, a set of methods, models and techniques were defined to cover, to a great extent, part of the development cycle of Web applications.

The methods developed within the context of the WE area emerged to provide support for the modelling of Web information systems. The original requirements for this sort of systems were storing, retrieving, transforming and presenting data to users. However, Web application requirements change over time and require more powerful and expressive methods for the development of systems that are not simply based on data but also in business processes (BP). Therefore, to provide support to these necessities, the methods for the development of Web applications have been extended and updated to provide the required mechanisms for the modelling of this kind of systems.

The set of methods considered in this analysis constitute the most relevant approaches within the WE area. These are UWAT+ (Distante, 2004), WebML (Ceri et al., 2000), OOHDM (Schwabe and Rossi, 1998), UWE (Koch, 2001), OO-H (Gomez et al., 2000), WSDM (Troyer and Leune, 1998), HERA (Houben, 2000) and 
MIDAS (Marcos et al., 2004). In particular, this analysis is focused on the solutions that each of these proposals provide to perform the construction of Web applications supporting BPs. This section has been organized as follows. First of all it presents each proposal by detailing the following points:

- Method description. This point provides a general description of each method that includes its origins, evolution and tool support.

- Adaptation to build BP-driven Web applications. This point presents the mechanisms defined by each method to carry out the construction of Web applications providing support to BPs.

- Development process. This point presents how each proposal has modified its development process to cope with the modelling phase related to BP.

Finally, once each proposal has been presented, section 3.1.9 provides a discussion about the support provided by the reviewed methods to deal with the requirements introduced by BP-driven Web applications (these requirements are detailed in section 2.3.3 from chapter 2).

\subsubsection{UWAT+}

The Ubiquitous Web applications (UWA) framework (UWA Consortium, 2002) is the result of a joint effort developed by a group of researchers from different countries which constituted the UWA Consortium (www.uwaproject.org). As its name stands for, this framework provides a methodology for the construction of 
ubiquitous Web applications. The ubiquitous aspect provides flexibility regarding the target device and the usage context in which Web applications are going to be used.

The objective of the UWA project was the production of an environment that would allow covering the development process of ubiquitous Web applications from requirements to the design phase. This consortium proposes the use of an extended and customized UML notation along the development process.

The aspects considered by the consortium for the definition of the framework include "Requirements Elicitation: Model, Notation and Tool Architecture", "Hypermedia and Operation design: model and tool architecture", "Transaction design" or "Customization design”.

Authors in (Distante et al., 2007) state that UWA provides a set of tools for describing ubiquitous Web applications. However we have not found any tool or even document where these tools were presented or explained.

\subsubsection{Adaptation to integrate process-driven Web applications}

Originally, the UWA framework did not face properly aspects such as (1) the necessity of describing the scenarios where process execution and navigation are mixed, (2) the specification of the set of contents and objects related to a particular activity or (3) the 
design of the collaboration of different users within a BP. Therefore, to deal with these limitations an extension called UWAT+ was proposed by (Distante, 2004). This extension focuses on the Transaction and Navigation Design phases of the development process.

On the one hand, the Organization and Execution Models built during the Transaction Design phase have been extended as follows:

- The Organization Model represents a web transaction from a static point of view. The main change introduced in this model allows differentiating the kind of relationships between activities. On the one hand, Hierarchical Relations allow specifying whether a sub-activity is required or optional to complete the associated activity. On the other hand, Semantic Relations allow specifying the dependence between two activities.

- The Execution Model represents a web transaction from a dynamic point of view. The main change introduced to the original model is that system activities are specified separately from user related aspects.

On the other hand, new diagrams have been included in the Navigational Model built during the Navigation Design phase. These diagrams are used as follows:

- The Activity Node concept was introduced to represent an elementary activity of a BP. This node is an interactive unit of information provided to the user in a single step. 
- The Activity Cluster concept specifies the possible navigation between a set of nodes. This navigation includes the interaction between process execution and content navigation.

In addition, the state of the process and its activities is kept in the system by means of a set of variables.

\subsubsection{Development Process}

UWA defines six models to carry out the specification of Web applications. First, following a goal-oriented approach, requirements are gathered in the (1) Requirements Model. Second, (2) Conceptual Model is built to specify the content of the application as well as the ways to access to it. In addition, with regard to the high-level transactional goals gathered in the requirements model, static and dynamic views of these requirements are defined in the (3) Organization and (4) Execution models respectively. These two models have been extended to deal with the specification of BPs (see section 3.1.1.1). Then, (5) the Navigational model is built to define the navigation throughout the content (Conceptual model) of the application. New diagrams have been included in this model to deal with the specification of BPs. Finally, the pages of the final Web application are designed in the (6) Publishing model.

The development process is made up of six phases which produce each of the models presented above. Figure 3.1 shows the 
development process of a Web application following the UWAT+ extension.

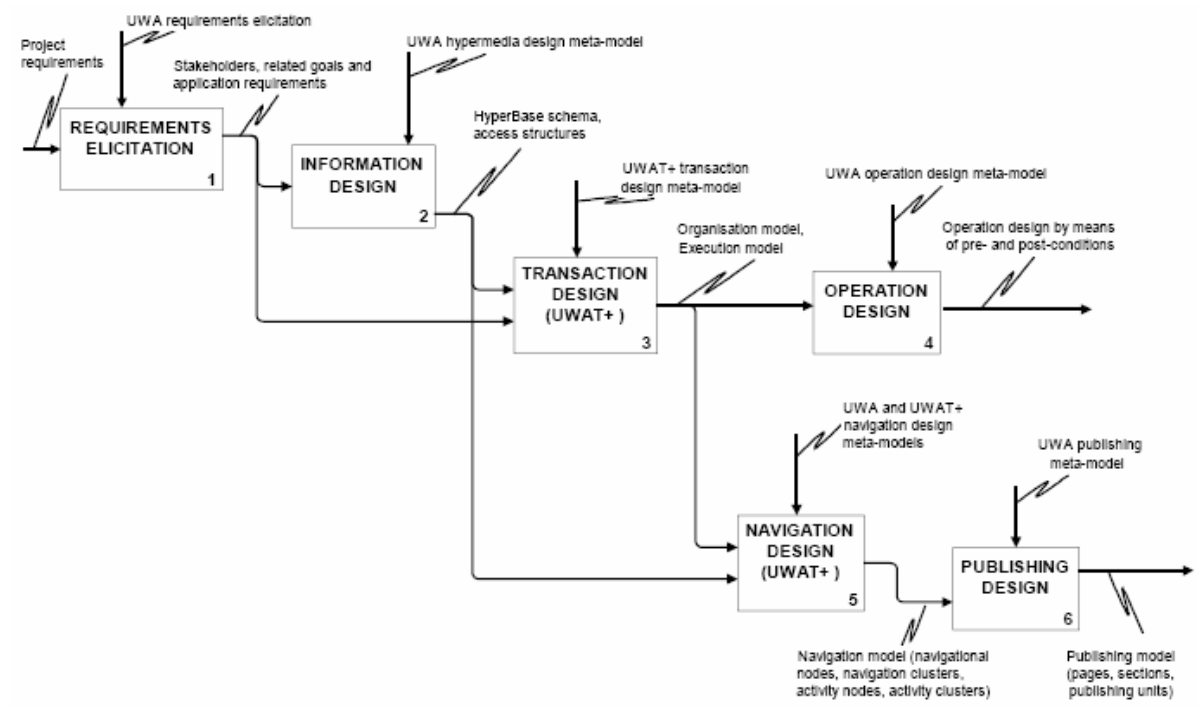

Figure 3.1 UWAT+ Development Process

In addition to the extensions defined to some of the models defined by UWA, the development process itself has also been modified regarding the order in which some steps are carried out. For instance, the Navigational design step is now performed after the Transaction Design phase. Moreover, the Conceptual model produced during the Information Design phase is used by the Transaction and Navigation Design phases.

\subsubsection{WebML: Web Modeling Language}

WebML (Ceri et al., 2000) was conceived in 1998 by an Italian research group ${ }^{11}$ from the Politecnico di Milano. WebML is a

${ }^{11} \mathrm{http} / / / \mathrm{www} \cdot$ webml.org 
notation for specifying complex Web sites at the conceptual level avoiding designers to commit detailed architectural details.

Originally, WebML was conceived to support the design and implementation of data-intensive Web applications; however, as a response to the new arising challenges appeared in the Web, WebML has evolved continuously to satisfy some of these new challenges. As a result of this evolution, WebML has been extended to address the generation of: web service-enabled (Brambilla et al., 2006b), workflow-based (Brambilla et al., 2006), context-aware (Ceri et al., 2007), semantic web (Brambilla et al., 2006a) and rich internet (Bozzon et al., 2006) applications. A complete description of this method gathering all these extensions is presented in (Brambilla et al., 2008).

Moreover, the WebML modelling language and its development method are implemented in a commercial Web tool called WebRatio Site Development Studio ${ }^{12}$. This tool covers the development process and automates the generation of the relational database and the application page templates. This tool allows fast-prototyping and can be also used for implementation, maintenance and evolution of the modelled Web applications.

12 http://www.webratio.com 


\subsubsection{Adaptation to integrate process-driven Web applications}

Web sites are described in WebML by means of four distinct orthogonal models which are (1) the structural model (which defines the data content by means of a compatible language with classical notations like the E-R model and the UML class diagrams), (2) the hypertext model which in turn consists of two submodels, the composition model (which defines the pages that compose the site) and the navigation model (which defines the topology of links between pages), (3) the presentation model (which defines the layout and graphic requirements for page rendering and (4) the personalization model (which defines the customization features for one-to-one content delivery).

However, none of the previously four presented original models were aimed at the development of workflow-based applications. Then, in order to deal with this limitation, WebML was extended to provide support to lightweight Web-enabled workflows (Brambilla, 2003; Brambilla et al., 2003; Brambilla et al., 2006). The proposed extension embraces both the language and the methodology itself. On the one hand, the language extension includes:

- The introduction of a new dimension (the BP model) into the methodology to deal with BP modeling. The notation used to deal with the BP modeling is the BPMN notation.

- The extension of the data model (see Figure 3.2) with a set of objects to describe the meta-data that is necessary for 
tracking the execution of the BP. This extension represents a reference model that gathers the process relevant information that is outside the data application.

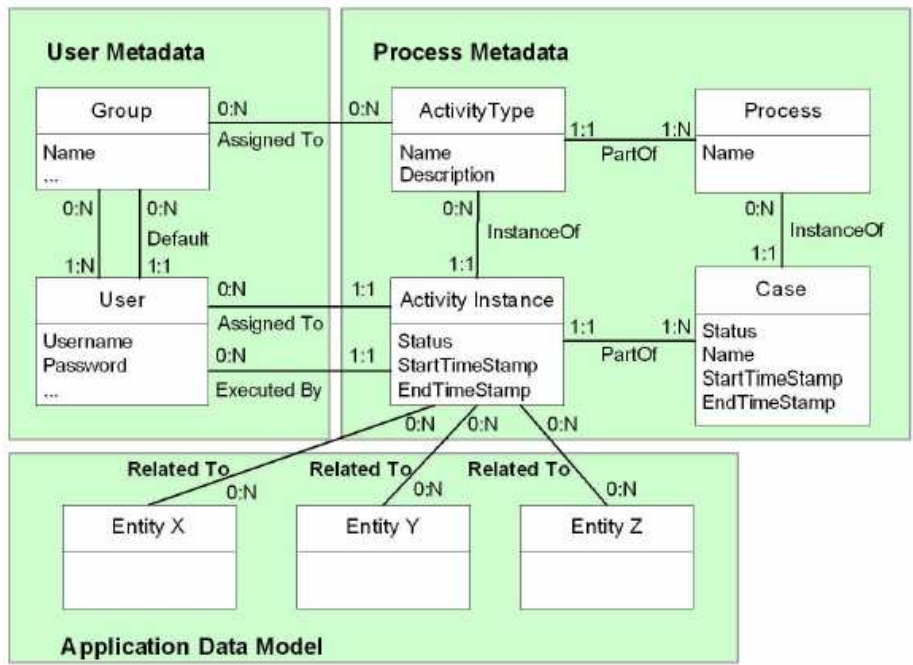

Figure 3.2 Data Model Extended

- The extension of the hypertext model to specify business activity boundaries and the workflow-dependent navigation links.

On the other hand, the extension regarding the methodology involves the following changes:

- New phases were introduced into the development process to cope with the modelling of BPs and their integration with the conceptual models.

- The design tool has been extended to allow the design of workflow models.

- New transformations for translating workflow diagrams into skeletons of Web applications defined in the WebML language. 


\subsubsection{Development Process}

WebML proposes an iterative and incremental development process (see Figure 3.3) where various tasks are repeated and refined until results meet the business requirements. This model allows the continuous testing, evaluation, modification or extension of each version of the system. As Figure 3.3 shows, the conceptual design phase has been extended with two new tasks (the process design and the process distribution) to endow the method for the generation of process-aware Web applications.

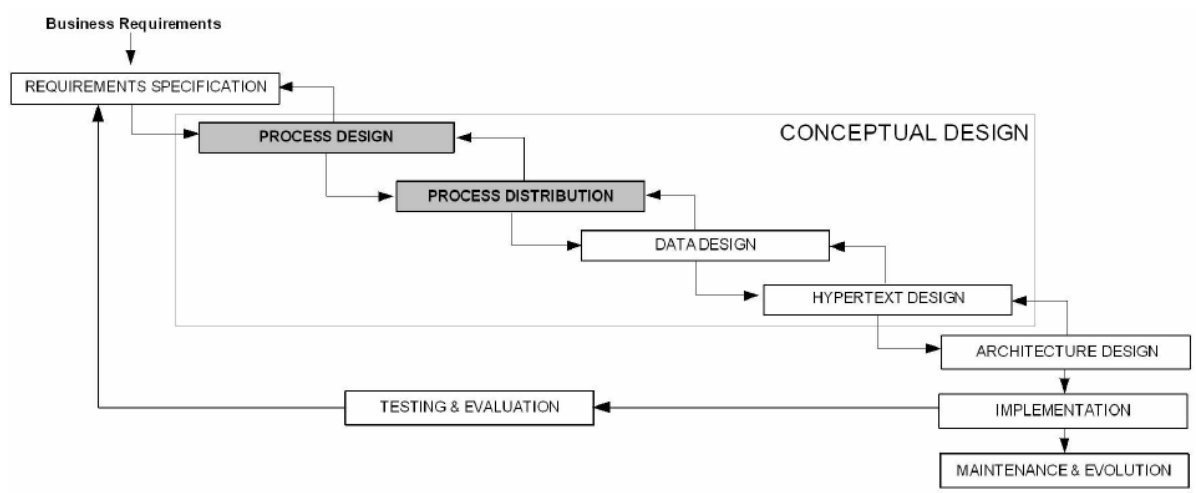

Figure 3.3 WebML Extended Development Process

- The process design task is focused on the high-level schematization of the processes underlying the application. Although the process design task is performed at the beginning of the conceptual design phase, this is not strictly immovable. If data is the central role of the application, the design task can be postponed after the data design task to fit better the application requirements nature.

- The process distribution task is focused on addressing the allocation of sub-processes to different peers, and therefore 
occurs only when there are several Web servers involved in the process enactment.

These two phases have influence over the data and hypertext design phases. This influence is clearly seen by the extensions performed to each of these models.

\subsubsection{OOHDM: Object-Oriented Hypermedia Design Method}

The OOHDM proposal was developed by Schwabe and Rossi in 1994 (Schwabe \& Rossi, 1998).

The set of models proposed by the OOHDM approach is based on object-oriented concepts, using concepts such as object structure and behaviour and abstraction mechanisms such as aggregation and generalization/specialization. However, although this method is based on object-oriented concepts, the resulting design can be implemented on top of any conventional platform.

The OOHDM method has evolved since its conception and it has been adapted to deal with the demanding necessities of each moment. Then, we find extensions to deal with Semantic Web technologies (Lima \& Schwabe, 2003; Schwabe et al., 2004), adaptation (de Assis et al., 2006), BPs (Schmid \& Rossi, 2004) and rich applications (Fialho \& Schwabe, 2007). A complete description of this method gathering all these extensions is presented in (Rossi \& Schwabe, 2008). 
The only available tool related to OOHDM is HyperDE ${ }^{13}$. However, this tool is based on the Semantic Hypermedia Design Method (SHDM) method, which defines an extension to the original method for the construction of semantic web applications.

\subsubsection{Adaptation to integrate process-driven Web applications}

The OOHDM method uses four models to specify hypermedia web applications. (1) The use case diagrams are used to provide a concise graphical representation of the interaction between the user and the system during the execution of a task. (2) The conceptual model represents the application domain. This model is based on well-known object-oriented modeling principles. (3) The navigational model describes the navigational structure of a hypermedia application. Expressing different views on the same domain is achieved by building different navigational models for the same conceptual schema. (4) Finally, the abstract interface model is built to map navigation with perceptible objects. It describes the interface for navigational objects and the responses to external events.

However, all these models were conceived basically to retrieve and manipulate the data of the system. Therefore, to deal with the modeling of BPs, OOHDM proposes to modify its conceptual and navigational model by bringing processes as first class citizens.

\footnotetext{
${ }^{13} \mathrm{http}: / /$ www.tecweb.inf.puc-rio.br:8000/hyperde
} 
This is achieved by adding concepts related to BPs in both models. On the one hand, the conceptual model is extended with a new primitive called activity (primitive abstracting the process activity concept). As a result, the conceptual model is made up of two types of classes, one referring to entities and another to processes. On the other hand, the Navigational model is extended consequently with a set of primitives related to processes. One of them is the called activity node, primitive that describes, in an abstract way, the visible attributes, anchors and operations, elements that will allow the user interacting with the system during process execution. Moreover, as activity nodes exist within the context of a particular process, these are contained within the corresponding process node (primitive that gathers related activity nodes). Finally, new types of links have been added to connect different activity nodes (navigation that does not leave the process) and activity and entity nodes (navigation that connects pure navigation with navigation during process execution). The semantics of navigation changes during the execution of a process (Schmid \& Herfort, 2004), and the new links are:

- Start: It allows the user moving from pure navigation to the execution of a process.

- Suspend: It allows the user leaving the execution of a process temporally. At this point, the process state is kept until it is taken again by the user.

- Resume: It allows the user taking up again the process execution from pure navigation. 
- Terminate: It allows the user leaving the process and changing to pure navigation. This stage is achieved because the process has been cancelled or finished.

Moreover, to specify the flow control of the processes, the proposal includes a new model into the method. This model uses the UML activity diagram notation (OMG, 2004) to specify processes.

\subsubsection{Development process}

OOHDM divides the development process into four steps which correspond to (1) domain design, (2) navigational design, (3) abstract interface and (4) implementation. During the first step a conceptual domain model is described using an object-oriented hypermedia design model. In this model primitive such as classes, relationships and sub-systems are used. In addition, a UML Activity Diagram has been added at this stage to define the control flow within processes. In the second step, the hypermedia of the application is described by means of navigational structures which are built based on the responsibilities assigned to different types of users. This model is partitioned in two sections which relate to pure navigation and process execution. Then, during the Abstract Interface Design step the specification about how users will perceive navigational objects through the interface is performed. Finally, during the Implementation step navigational and abstract interface models are transformed into a concrete implementation environment. 


\subsubsection{UWE: UML based-web Engineering}

UWE (Koch, 2001; Koch \& Kraus, 2002) came up by 1998. The method was developed by the WE Group from the LudwigMaximilians-Universität München.

It is a software engineering approach based on UML (OMG, 2004) (it uses the UML standard notation as much as possible) and defines a UML profile to specify the peculiarities that introduce web applications. The major benefit of being UML compliance is that any CASE tool that supports the UML notation can be used to produce the UWE models.

The UWE approach has evolved to deal with new features such as personalization or support for BP execution (Koch et al., 2004; Knapp et al., 2004). In addition, the approach has also evolved regarding technological issues such as adopting aspect-oriented techniques or model driven principles (Koch, 2006; Kraus et al., 2007; Koch, 2007). (Koch et al., 2008) provides a complete description of the approach including all these extensions.

An extension to the open-source CASE tool ArgoUML ${ }^{14}$ has been implemented to provide modelling support for UWE (the development process is not realized within the tool). This tool is called ArgoUWE ${ }^{15}$ and supports the UWE method during the

\footnotetext{
14 http://argouml.tigris.org/

15 http://www.pst.informatik.uni-muenchen.de/projekte/argouwe
} 
modelling phase. However, the code generation phase in which the Web application is built is out of the scope of this tool.

\subsubsection{Adaptation to integrate process-driven Web applications}

UWE proposes to deal with the process of modelling web applications with a set of models to specify four different aspects. (1) Initially, to deal with requirements, UWE proposes the use of Use Cases and UWE Activity Diagrams. The former is used to specify users of the web system and the functionality that the system will provide to them. The latter is used to provide a more detailed description (pre and post conditions, a workflow description, exceptions, etc.) of the functionalities specified previously. (2) The content model provides a visual specification of the domain application. In addition, to deal with customization aspects (adaptation to the properties of users or user groups to features of the environment) this model is accompanied with the user or context model. Both models are graphically represented by a UML class diagram. (3) The navigational structure allows defining the navigation nodes and links, menus, access primitives (indexes and guided tours), etc.

The extension defined by UWE involves the phases related with requirements and the navigational structure. Moreover, it proposes introducing a new model that allows defining the structural and behavioural view of the process. This model is called Process flow model and allows modelling processes 
independently of navigation, achieving the always wanted separation of concerns in the design of Web applications. An important difference between this model and the navigational one is that in this case, the model can include elements that are not derived from the conceptual model.

At the requirements stage, UWE extends the Use case modelling technique by differentiating two types of use cases, the Navigation Use cases and the Process Use cases.

- Navigation Use cases are used to model typical user behaviour when interacting with a web application (browsing or searching some content).

- Process Use cases are used to describe business tasks that end users will perform with the system.

On the other hand, UWE proposes enriching the navigation structure to reflect the set of integration points where the user leaves pure navigation to change into the view referred to process execution. To achieve this goal, the proposal has been extended with the inclusion of two new stereotypes, which are the Process Class and the Process Link primitives.

- Process Class: This stereotype represents classes whose instances are used by the user during the execution of a process. It is possible to define a mapping function between process classes and use cases (those related to processes) similarly to the mapping functions defined between navigational classes and conceptual classes.

- Process Links: This stereotype represents the association between a navigational class and a process class. Moreover, 
this kind of links needs to have associated information about the state of the process (i.e. links must be restricted by an OCL expression about the state of the process). This allows taking up again activities within a process under certain conditions.

\subsubsection{Development process}

The construction of the navigational model is performed in two steps. The former consists in building the navigational model that includes both navigational and process classes which have been derived from the Use cases built in the analysis stage. The later step consists in the introduction into the navigational model of a set of access structures which are needed to complete the navigation. This step is partially automated and consists in the introduction of indexes, tourist guides and queries following certain rules. The process structural model similar to the navigational model is derived from the conceptual model. The developer must determine the set of conceptual classes that are relevant to the process flow. The Process flow model is a refinement of the process model defined during the analysis level and represented by means of UML activity diagrams. UWE describes by means of this model the process behaviour (sequence of activities that make up the process). Each activity from the Process Flow Model defines either a UML subactivity representing the execution of a non atomic sequence of steps or a UML call representing an atomic action that invokes to just one operation. This operation is always referred to operations defined in the process classes included in the structural process model. UWE, in 
line with its philosophy of being UML compliance, follows strictly the notation and semantics defined by the UML activity diagram.

\subsubsection{OO-H: Object-Oriented Hypermedia Method}

OO-H is an initiative started in 2000 by Gómez et al. (Gómez et al., 2000; Cachero \& Gómez, 2002). OO-H was originally defined as an approximation addressed by the user requirements, object oriented-based and partially based on the standards. Based on the object-oriented paradigm, this approach provides designers with the semantics and notation necessary for the development of personalized Web-based interfaces.

The OO-H proposal has been extended to deal with (1) dynamic personalization (Garrigos et al., 2003) studying how personalization properties influence navigation and presentation models and (2) BPs integration (Koch et al., 2004).

The analysis and design steps proposed by OO-H are supported by a modelling environment called VisualWADE ${ }^{16}$. This tool provides a set of model compilers that allow generating a running application for different platforms and languages.

\subsubsection{Adaptation to integrate process-driven Web applications}

OO-H proposes to deal with the modelling process with three different models. These models are (1) the conceptual model, (2)

16 http://www.visualwade.com 
the Navigational model and (3) the Presentation model. The conceptual model is defined by means of a UML class diagram that keeps information related to the domain object. The Navigational model is defined by means of the Navigational Access Diagram (NAD) which allows defining menus, navigation, views over conceptual classes and the paths provided to the user to traverse the system. Finally, the Presentation model is used to define the characteristics of each navigation path. These characteristics allow changing certain visibility features such as different link colours.

OO-H proposes the complementary use of UML activity diagrams to represent the process control flow, endowing the expressive power of the $\mathrm{OO}-\mathrm{H}$ to allow the modeling not uni-granular (atomic) and multi-granular (compound) services. The integration is performed by preserving the navigational model and ensuring the accomplishment of the restriction imposed by the control flow of processes. Therefore, OO-H proposes mapping the elements defined in the process model with elements from the navigational model.

\subsubsection{Development process}

OO-H proposes five steps for the development of web applications that support BPs. (1) Initially, the developer must refine the conceptual model by adding properties and even new elements to the UML class diagram. (2) Then, the developer must refine the process model defined at the analysis level by means of Use cases. Taking in mind the previously refined conceptual model, it is 
possible to build a more detailed activity diagram where the links between the constructs of the process design and the conceptual model is established by the identification of UML call states (atomic actions that would invoke the execution of only one operation). (3) The designer must then apply a set of predefined mapping rules that allow the automatic generation of Navigation Access Diagrams (NAD) based on the design of process models. This automatic generation ensures the traceability between both models (navigational and process models). (4) Then, the designer must perform again over the recently obtain NAD diagram the necessary refinements such as the addition of filters or extra navigational paths. (5) Finally, once the navigational model is completed, the designer can continue the process by defining presentation aspects of the system.

\subsubsection{WSDM: Web Site Design Method}

WSDM is an initiative developed in the Vrije Universiteit Brussel (Troyer \& Leune, 1998). WSDM is a methodology and as such, it provides modelling primitives and a systematic way to develop web sites/applications.

Originally, the WSDM abbreviation referenced a method for the design of Web sites (Web Site Design Method). However, the method has evolved allowing now not only the design of traditional web applications but also the development of semantic web applications. For this reason, the abbreviation now stands for Web Semantics Design Method. 
WSDM has been extended to deal with new modelling requirements. The most important extensions performed to the approach are to support (1) localization (Troyer \& Casteleyn, 2004) and adaptation (Casteleyn, 2005). A complete description of this method gathering all these extensions is presented in (Troyer et al., 2008).

WSDM does not have any tool that provides support either to the method or to the models defined to deal with the description of web applications.

\subsubsection{Adaptation to integrate process-driven Web applications}

The extension proposed by WSDM involves mainly the Conceptual design phase which in turn is constituted by the Task and Information Modelling and the Navigational design phases.

On the one hand, the Task Modelling phase endows the method to model explicitly tasks. To deal with the modelling of tasks WSDM uses the ConcurTaskTree (CTT) notation (Paternò et al., 1997). This notation was developed in the context of the HumanComputer Interaction (HCI) to describe user tasks. This notation allows specifying four different types of tasks (user, application, interaction and abstract tasks) as well as the temporal relationships between these. However, WSDM does not follow completely the original CTT notation. In fact, it has been adapted and slightly modified (user tasks are discarded because these are 
considered not useful during the design stage and some operators have modified their meaning to express temporal relationships between tasks) to satisfy the particularities that introduced the design of Web applications.

On the other hand, the navigational model allows now a better separation of concerns between the structure and the process workflow. In this case, the task navigational model allows describing the way in which users can perform tasks in the web site. This model is built from the model task and the object chunks defined during the previous stage of task modelling. Components and Project logic links are used to build the task navigational model for each task model defined previously. However, to express the workflow or the process logic defined in the task model by means of temporal relationships in the CTT they use what they call Process logic links.

\subsubsection{Development process}

WSDM proposes to deal with the modelling process in five phases which, in some cases, are made up in turn of different phases. The first phase is the Mission Statement Specification whose goal is the identification of the purpose of the web system (the proposal follows an audience-driven design approach) as well as the subject and the target users. Then, the Audience modelling phase comes into play. In this phase, the target users identified in the previous phase are refined into audience classes. The next phase is the Conceptual Design phase. In this phase the method specifies the information, functionality and structure of the web system at the 
conceptual level. On the one hand, the information and functionality are specified during the Task and Information modelling sub phase. On the other hand, the navigational possibilities for each audience class are specified during the Navigational design sub phase. Then, it is time to perform the Implementation Design phase. This is made up of three sub phases which are Site Structure Design (in this phase the conceptual structure of the website is mapped into pages), Presentation Design (in this phase the look and feel of the web system as well as the layout is defined) and Logical Data design (this phase is only performed for data-intensive systems and is aimed at the construction of a database schema and the mapping between the conceptual data model and the actual data source). Finally, the Implementation phase is reached, which is performed by using the set of models generated previously.

\subsubsection{HERA}

HERA (Houben, 2000; Houben et al., 2003; Houben et al., 2004) is a method resulting of the Hera research project ${ }^{17}$ started in 2000 initially at the Technische Universiteit Eindhoven and supported later by other Belgium fellow researchers from the Vrije Universiteit Brussel and the University of Namur. It is a method for the design of Web information systems that found its origins in an approach for hypermedia presentation generation.

17 http://wwwis.win.tue.nl/ hera/ 
The approach has been subject of different refinements resulting in the Hera-S (van der Sluijs et al., 2006) extension. This extension includes compliant models to the original ones (hypermedia-based) which are based on the Resource Description Framework (RDF) and RDF Schema (RDFS). This extension was defined to easy the development of web systems when these rely on very heterogeneous data sources. A complete description of this method gathering all these extensions is presented in (Houben et al., 2008).

Hera Studio is a graphical design tool that supports the domain, context and application models defined by the proposal. Each of these models is built in a separate editor and when these are completed, these can be exported into an RDF serialization, format that can be used by Hera. Currently, the application model editor is being extended to support some of the specific constructs introduced by the semantic extension (HERA-S).

\subsubsection{Adaptation to integrate process-driven Web applications}

The method defines three main models which are targeted to model (1) content, (2) hypermedia navigation and (3) presentation. The Domain Model (DM) describes the structure of the content data. Based on this model, the Application Model (AM) is built. This second model describes a hypermedia-based navigation structure over the content. Moreover, HERA allows, by means of the Context Model (CM), the dynamic personalization and 
adaptation of the content. Then, the AM is instantiated resulting in an Application Model Pages (AMP) which can be though of as pages that display data and that contain links that allow moving from one to another AMP. Then, AMPs are transformed into suitable presentations by a presentation engine.

To carry out the modeling of BPs, HERA introduces the Workflow Model into the already presented models. The goal of this model is to describe the set of BPs of the system as well as the collaboration with both users and external systems. This model is defined by means of UML Use cases and Activity Diagrams, which have been extended with a language (based on ECA rules) for detailing the activity states.

\subsubsection{Development process}

HERA proposes a development process consisted in six phases. (1) Initially, an identification of actor representing user groups or external systems and their assigned tasks is performed by means of the task model. This specification is performed using a Use case diagram. (2) Then, the Domain model is built to specify the application domain data structure. (3) Regarding the workflow of the system, this is defined in the Workflow model. (4) Then, state activities are specified in the forth step. (5) Once all the requirements are expressed in the corresponding models, an automatic transformation can be executed to generate a model describing the internal data processing and the basic navigation structure of the previously designed system. (6) Finally, an optional step can be performed to better fit the generated models. 


\subsubsection{MIDAS}

MIDAS (Marcos et al., 2004) is a Spanish initiative developed by researchers from the Kybele Research Group at the Rey Juan Carlos University.

MIDAS is a methodological framework that proposes the development of Web Information Systems based on the MDA approach. For this reason, it proposes to perform the modelling process according to two dimensions. The first dimension is related to the MDA abstraction techniques (CIM, PIM and PSM) and how these are mapped to each other. The latter is related to the hypertext (Caceres et al., 2004), content (Marcos et al., 2003) and behaviour (Marcos et al. 2003a) relevant aspects in Web Information System (WIS) development.

This is the most recent proposals from those analyzed in this section. For this reason, the support to cope with BPs is provided from the beginning, being not necessary the definition of any extension.

The models proposed by MIDAS are supported by the MIDASCASE tool. This tool includes a verification and validation module that informs users about the completeness and correctness of the defined models. 


\subsubsection{Adaptation to integrate process-driven Web applications}

MIDAS proposes a set of models for WIS development which are organized orthogonally in the abstraction levels defined by the MDA approach (CIM, PIM and PSM) and in the aspects that characterize WIS systems (hypertext, context and behaviour).

In order to deal with BPs, MIDAS defines five models which are gathered in the behaviour aspect and distributed in the three different abstraction levels defined by the MDA approach. The (1) Business model is built by the analyst and it is used to capture the requirements in the form of BPs. Then, in the (2) User Services model, the developer identifies the users of the system as well as the services (understood as complex functionality that satisfy a specific need of the user) that the system will offer them. Once, users and system services have been identified, by means of the (3) Extended Use Cases model, the developer refines each of the previous identified services in one or more user services, which in turn can be defined as basic or composite services. Basic services are also specified as structural (services aimed just for showing pieces of information) or functional (services aimed to represent interaction between the user and the system).Then, to define the flow of the previously identified services, the (4) Services Process model is built. The notation used in this model is the UML activity diagram and each activity is mapped to the basic services defined in the Extended Use Cases model. To detail more precisely the service processes defined in the previous presented model, the (5) 
Services Composition model is built. At this stage, the developer (a) identifies the set of business collaborators that participate in the process, (b) splits service activities into activity operations (activities carried out by collaborators), (c) identifies which activity operations will be provided as Web services and (d) distributes the activity operations between the identified collaborators.

Related to the Hypertext aspect, MIDAS defines two models at the PIM level. On the one hand, in the (1) Extended Slice model, the system is decomposed into significant parts (slices, which in turn can be defined as structural or functional, and hyperlinks). This model could be considered as a view over the Conceptual Data model where the developers filters the data and functionality that is going to be shown in the user interface. On the other hand, (2) the Extended Navigation model is based on the previous model and includes navigation structures such as Menu, Index or Query among others. This model defines how the slices defined in the Extended Slice model are going to be provided to the user.

\subsubsection{Development process}

The development process proposed by MIDAS to perform the development of process-driven Web applications follows the development process depicted in Figure 3.4. 


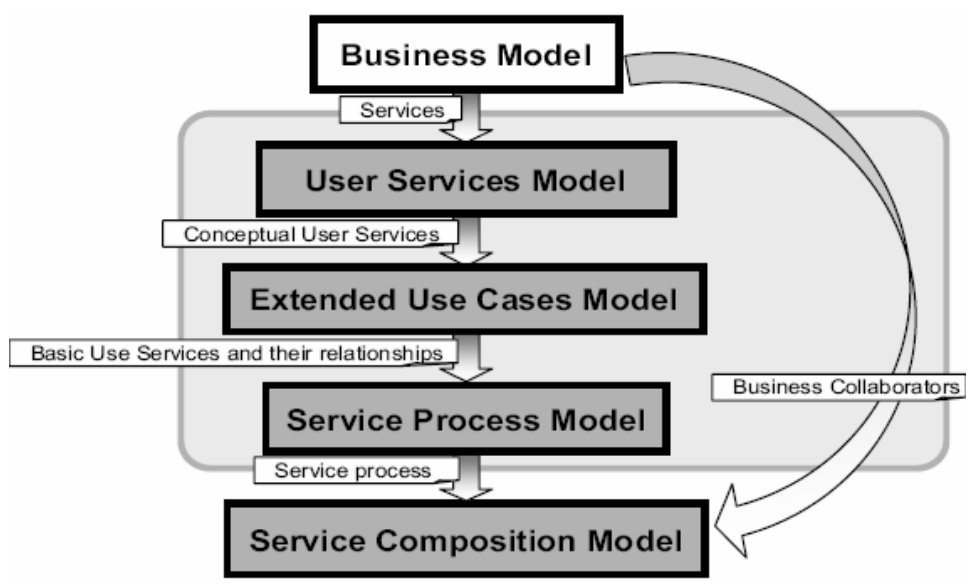

Figure 3.4 MIDAS development process focused on BP

This figure only shows the set of models included in the behaviour aspect. However, the Extended Slice model defined within the Hypertext aspect is derived from the Extended Use Cases Model and the Conceptual Data model defined in the content and behaviour aspects respectively.

\subsubsection{Discussion}

This section summarizes the support provided by the reviewed methods to deal with the requirements that WE methods should satisfy for building BP-driven Web applications (these requirements are presented in Chapter 2). This summary is organized as follows. First, the support provided by the reviewed proposals is discussed. Then, we outline the solution designed in this thesis to satisfy the identified requirement.

Requirement 1. Differentiate between Short-running and Longrunning Processes. 
From the set of reviewed methods, HERA, UWAT+ and WebML provide modelling mechanisms for specifying both types of BPs. However, none of these methods follows a code generation strategy that allows producing Web applications in which users can differentiate the type of process being executed. The main reason to make this differentiation explicit is to help users in completing the $\mathrm{BP}$ in which they are involved.

\section{Solution Proposed in this thesis:}

In this scenario we propose the generation of a different GUI depending on the case. On the one hand, short-running BPs are presented to users similarly to a wizard. The involved user follows a set of steps which allows the accomplishment of the BP goal. On the other hand, long-running BPs are presented to users similarly to the way BPMS do. In this case, processes are handled throughout a TODO list. This list allows users reaching and completing their pending tasks easily. In addition, usability aspects are also considered for the generation of these GUIs. These are taken into account in order to improve the user experience which results in speeding the completion of process tasks.

Requirement 2. Specify the data and functionality to support BP activities.

All the reviewed methods provide mechanisms to specify the functionality that supports the execution of BP activities. However, regarding with the data necessary to complete $\mathrm{BP}$ activities, only OOHDM and UWAT+ provides mechanisms (by 
suspending and resuming tasks) that allow users moving from BP navigation to content navigation to reach this data.

Solution Proposed in this thesis:

In this thesis, views over classes and services defined in the Structural and Services model respectively allows us defining which data and functionality is going to be presented to the user to complete process tasks. One of the extensions performed to the BPMN notation is to associate process tasks with functionality that is defined either in the Structural or in the Services model. Therefore, by means of the association with the Structural model we are implicitly defining which classes are associated to each activity.

Requirement 3. Deal with the Specification of BP flows.

All the reviewed methods provide mechanisms to specify BPs flow. All of them make use of specific languages or notation to represent these flows (i.e., BPMN, UML Activity Diagrams or Concur Task Tree).

\section{Solution Proposed in this thesis:}

In this thesis we have introduced a new model to specify the BP flows. This model is called the Business Process Model and is based on the BPMN notation. The expressivity provided by this model (i.e. fork and merge gateways or loop activities) allows us representing graphically the potential paths that can be taken during business process execution. 
Requirement 4. Deal with Distributed Processes.

Only the HERA, MIDAS and WebML consider the collaboration with external parties to support some of the activities included in the BPs. On the contrary, OOHDM, OO-H, UWE, WSDM and UWAT+ conceive web applications as isolated systems where all the activities of the BPs is supported by the own Web application.

Solution Proposed in this thesis:

This requirement is satisfied by means of the notation that has been used to represent BPs which is BPMN (see chapter 2 for details on the notation). This notation provides graphical elements (specifically the Pool and the Message Flow graphical elements) that allow specifying different organizations involved in the same $\mathrm{BP}$ and the way they cooperate to accomplish the BP goal.

Requirement 5. Deal with Multiple roles.

Most of the analyzed proposals (MIDAS, OOHDM, OO-H, UWE, and WSDM) just deal with the specification of short-running BP. Therefore, even though the notations used to model BPs provide mechanisms for specifying different roles, this mechanism is not used by these methods. Other methods such as WebML, HERA and UWAT+ allow the definition of different roles which participate for the conclusion of a specific BP.

\section{Solution Proposed in this thesis:}

Similarly to the previous requirement, this is satisfied by means of the BPMN notation. In this case, the graphical elements provided by the notation (the Lane and the Flow graphical elements) allow 
specifying different responsibilities within the same organization and the way they cooperate to accomplish the BP goal.

Requirement 6. Deal with Manual Tasks.

None of the analyzed proposals take into account that BPs can define tasks that are performed manually.

Solution Proposed in this thesis:

Similarly to the two previous requirements, this is satisfied by means of the BPMN notation. In this case, BPMN provides with different types of activities which include the "manual" type activity. This type is used to represent manual tasks included in the BP specification. Moreover, a proper GUI is provided to the user to accomplish this type of task.

Requirement 7. Separation of Concerns related to Navigation.

The analyzed proposals deal with the separation of concerns regarding navigation at three different levels:

a) By describing the control flow of the BPs by the use of UML activity diagrams or a similar language. All of the proposals make use of this mechanism.

b) By introducing new primitives into the navigational model to differentiate content navigation from navigation during $\mathrm{BP}$ execution. This mechanism has been adopted by the OOHDM, UWAT+, UWE, WebML, MIDAS and WSDM proposals. However, proposals such as HERA and OO-H simply use the existing navigational primitive to simulate $\mathrm{BP}$ execution. 
c) By introducing mechanisms that allow leaving the execution of a BP (suspending the current BP instance) and starting with content navigation and vice versa (resuming the suspended BP instance). This aspect has only been considered by the OOHDM and UWAT+ proposals.

\section{Solution Proposed in this thesis:}

Although these mechanisms constitute a first attempt to distinguish navigation in both cases (content navigation and BP navigation), it still requires defining the navigational flow within the navigational model. This decoupled degree can be acceptable for the definition of simple processes. However, this solution does not scale for more complex situations where the control flow derives into complex structures that are difficult to handle within the navigational model. Therefore, we propose to further the separation of concerns by maintaining the navigation that occurs during $\mathrm{BP}$ execution inside the definition of the process. To achieve this level of separation of concerns we have extended the architecture of the generated Web applications by introducing a process engine that is capable of handling the execution of BPs.

Requirement 8. Keep the State of the BP.

On the one hand, UWAT+ and WebML have extended their respective conceptual models to keep explicitly the state of the started activities and the BP. This mechanism allows these proposals to restore the process state whenever this is necessary. On the other hand, methods such as OOHDM, UWE, OO-H, MIDAS, HERA and WSDM keep the state of the BP implicitly in 
the navigational model. The main problem with this approach is that the state of a started BP cannot be restored if the user has left the BP (i.e., when the computer is switched off unintentionally).

\section{Solution Proposed in this thesis:}

In this work we propose to keep the state of the BP explicitly. However, in contrast to the solutions proposed by UWAT+ and WebML, in this work we make use of a process engine that is in charge, among others tasks, of keeping the state of each launched BP.

In addition to these requirements, even though the existence of a tool supporting the method does not change the method itself, it provides an added-value to the method and contributes to the acceptability of the method in industrial environments (Garzotto $\&$ Perrone, 2007). For this reason we are also going to mention the tool support provided by the reviewed methods.

\section{Tool support}

Only WebRatio and ArgoUWE (tools supporting WebML and UWE respectively) provide support to the extension to deal with $\mathrm{BP}$ integration. However, in the case of ArgoUWE, this support is limited to the modelling stage. On the contrary, WebRatio processes the models defined in the proposals to produce the running Web application. 
Regarding the HERA, UWAT+, OOHDM, OO-H, MIDAS and WSDM proposals, up to date there is no tool supporting the extensions proposed by each of them.

Solution Proposed in this thesis:

In this thesis, we have developed an Eclipse-based tool that supports the modelling and the transformation phases that allow building a BP-driven Web application based on the specification performed at the modelling level. This tool combines different projects developed within the Eclipse environment (EMP and STP) that have allowed its construction.

Table 3-1summarizes the support provided by each of the previously analyzed proposals to deal with the integration of BPs in Web applications.

\begin{tabular}{|c|c|c|c|c|c|c|c|c|}
\hline & $\underbrace{+}_{5}$ & 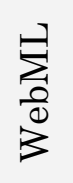 & $\begin{array}{l}\text { 亥 } \\
\text { 完 } \\
0\end{array}$ & $\frac{1}{3}$ & $\begin{array}{l}\text { Tr } \\
8\end{array}$ & $\begin{array}{l}\sum_{1} \\
\text { On } \\
3\end{array}$ & 胥 & $\frac{\pi}{\stackrel{\Xi}{\mid}}$ \\
\hline Requirement 1 & 1 & 1 & $\bullet$ & $\bullet$ & • & - & 1 & - \\
\hline Requirement 2 & 1 & I & • & । & । & । & 1 & 1 \\
\hline \begin{tabular}{|l|} 
Requirement 3 \\
\end{tabular} & I & I & - & । & । & । & I & I \\
\hline Requirement 4 & - & 0 & • & $\bullet$ & • & • & 0 & 0 \\
\hline Requirement 5 & 0 & o & - & $\bullet$ & $\bullet$ & • & o & - \\
\hline Requirement 6 & • & $\bullet$ & $\bullet$ & • & $\bullet$ & • & $\bullet$ & - \\
\hline Requirement 7 & 1 & ! & ! & $\bullet$ & $\bullet$ & 1 & $\bullet$ & 1 \\
\hline Requirement 8 & 0 & o & - & । & ' & ! & 1 & 1 \\
\hline
\end{tabular}

Table 3-1 WE methods Summary 
The symbols used in Table 3-1 have the following meaning:

O: The approach completely supports the feature

I : The approach partially supports the feature

: The approach does not support the feature

\subsection{Conclusions}

In this chapter we have presented the most well-known approaches developed within the WE area and how these have faced the integration of BPs into Web applications. First we have introduced each proposal presenting its origins and the set of extensions developed to deal with the different requirements that have emerged since the birth of the WWW. Then, we have presented the mechanisms proposed by each proposal to deal with the integration of BPs at the modelling level and how this affects to the corresponding development process. Once all the proposals have been presented, a discussion about the appropriateness of each solution is provided. This discussion is based on the requirements that Web Engineering methods should support to build BP-driven Web applications (these requirements were presented in chapter 2). This discussion reveals that some of the requirements are not addressed properly and others even are not considered. Finally, the discussion has been completed by providing an overview of the solution developed in this thesis to deal with each requirement. 



\section{Chapter 4}

\section{Development Process}

This chapter presents the development process that has been designed to deal with the construction of BP-driven Web applications. This process extends the process defined by the OOWS method by introducing and modifying some of the existing steps. Along the chapter, each step of the process is described in detail specifying the set of artefacts (model or code) that are required and that are produced by each of them.

The chapter has been organized based on the phases that relate to the extension. On the one hand, during the Design phase the system is represented in terms of the models defined by the method. On the other hand, during the Implementation phase, by means of the application of model transformations, we obtain automatically an equivalent representation of the system but this time in terms of an implementation language.

\subsection{Introduction}

The Model Driven Development (MDD) approach proposes to deal with the software development process based on the use of models. Putting this approach into practice involves three steps. First of 
all, it is necessary to define a language (metamodel) that allows expressing a particular kind of systems. Then, in a second step, this language can be used to create models that represent different system instances. Finally, in order to execute these models it is necessary to transform them into an executable representation. This last step is achieved by means of model transformations which allow moving the system represented in the models into a particular implementation technology. But these are the ingredients that make possible the application of the MDD approach. However, it is necessary to establish the steps that define the development process as well as the artefacts resulting from each step.

Independently of the software development model used in the definition of a software development process (waterfall or iterative), a complete process involves six different steps which covers from requirements gathering to software maintenance. However, in this chapter, the development process is focused just on two of these steps which relate to the Design and Implementation of the Web system. This simplification is due to the fact that the proposal developed in this thesis has only impact in these two steps.

Therefore, according to this simplification of the development process, the proposed process is made up of two steps. During the first step, the Design of the Web system is performed. In this case, the system is represented in terms of the models defined by the method (structure, behaviour, system views, etc) (see section 4.3). 
Then, in a second step, all these models are transformed into a particular implementation technology, allowing the execution of the modelled system (see section 4.4). The latter step is performed by the application of model transformation techniques which allow moving from the problem space (real world concepts) to the solution space (system implementation).

Before presenting the development process itself, in the following subsection we advocate for the use of the BPMN notation to represent graphically the development process.

\subsubsection{Using BPMN to Define the Development Process}

There are different approaches that can be used to specify software development processes. Some of these approaches are the PIE methodology (Cunin et al., 2001), the OPEN Process Framework (Firesmith \& Henderson-Sellers, 2001) or SPEM (OMG 2005b). However, since in this work we use the BPMN notation to describe business processes, we find more appropriate to use this notation to specify the development process. In fact, in (Sousa et al., 2007), authors proved that BPMN can be used to represent graphically methods defined according the SPEM specification. This proof is based on a comparison made between the elements defined by SPEM and the ones defined by the BPMN notation. This comparison shows that it is possible to find BPMN elements to represent each of the elements defined by SPEM.

However, since the BPMN notation defines different types of submodels to create BPs, we need to specify the use made of this 
notation to define the development process. The different types of sub-models provided by the BPMN notation are "private (internal) business processes", "abstract processes" and "collaboration processes". From these types, we have used the "private (internal) business process" type of sub-model. In this case, the internal business processes refers to the development process itself. Although the expressivity provided by the BPMN notation is enough to describe the whole process (it includes "Flow Objects", "Connecting Objects", "Swimlanes", “Artefacts", "Events", "Activities", "Gateways", etc.), we still need to differentiate the type of document produced in each task. For this purpose we have extended the semantics of the Data Object graphical element from the original BPMN notation with the use of stereotypes. Therefore, when the generated artefact refers to a model, we will denote it with the "model" stereotype. On the contrary, if the generated artefact corresponds to executable code we will denote it with the "code" stereotype. In addition, the process tasks in gray colour represent the tasks that have been introduced or modified in the current proposal from the ones defined by OOWS originally.

\subsection{The Big Picture}

The process depicted in Figure 4.1 shows the development process resulted after extending the OOWS method to deal with the execution of Business Processes. As this figure shows, the process involves the participation of three different roles, two of which are related to human-beings (the Analyst and the Developer) and the 
third to a system (the Bizzy tool) as indicated by the stereotypes "human-being" and "system" respectively in Figure 4.1.

The process is started by the Analyst. This user defines, using the BPMN notation, the set of Abstract ${ }^{18}$ Business Processes (ABP) that have to be supported by the system ("Business Process Analysis" task). As a result of this task a set of ABP are produced.

Then, the Developer appears in the process. Based on the previously generated $\mathrm{ABPs}$, this participant represents these BPs in terms of the OO-Method models and the Services model (see Figure 4.2 the "System Specification" sub-process task). As a result of this task, an OO-Method plus a Services model representation of the BPs is obtained.

Although the development process proposes performing first the "Business Process Analysis" and then the "System Specification", these tasks can be performed in the other way round. The order in which these tasks are going to be performed is determined by the way in which the system requirements ${ }^{19}$ are discovered. In some cases, requirements are provided in terms of a well defined process. In other cases, requirements are provided in terms of domain concepts and uni-granular functionalities.

\footnotetext{
18 This process description is abstract since it cannot be executed in a process engine. It is an incomplete BP definition from the execution point of view.

${ }^{19}$ In this case we are referring to functional requirements.
} 


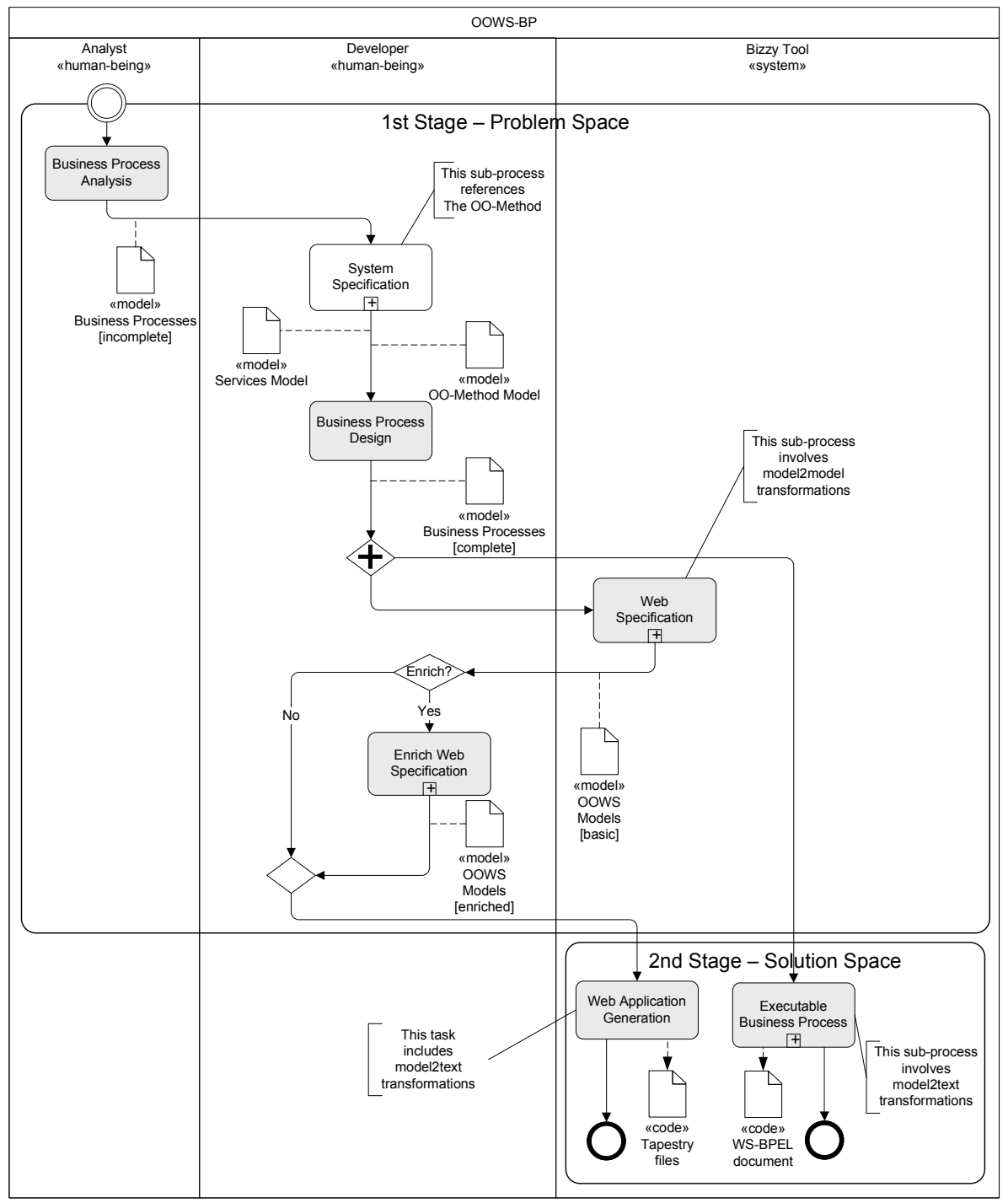

Figure 4.1 Process Development 


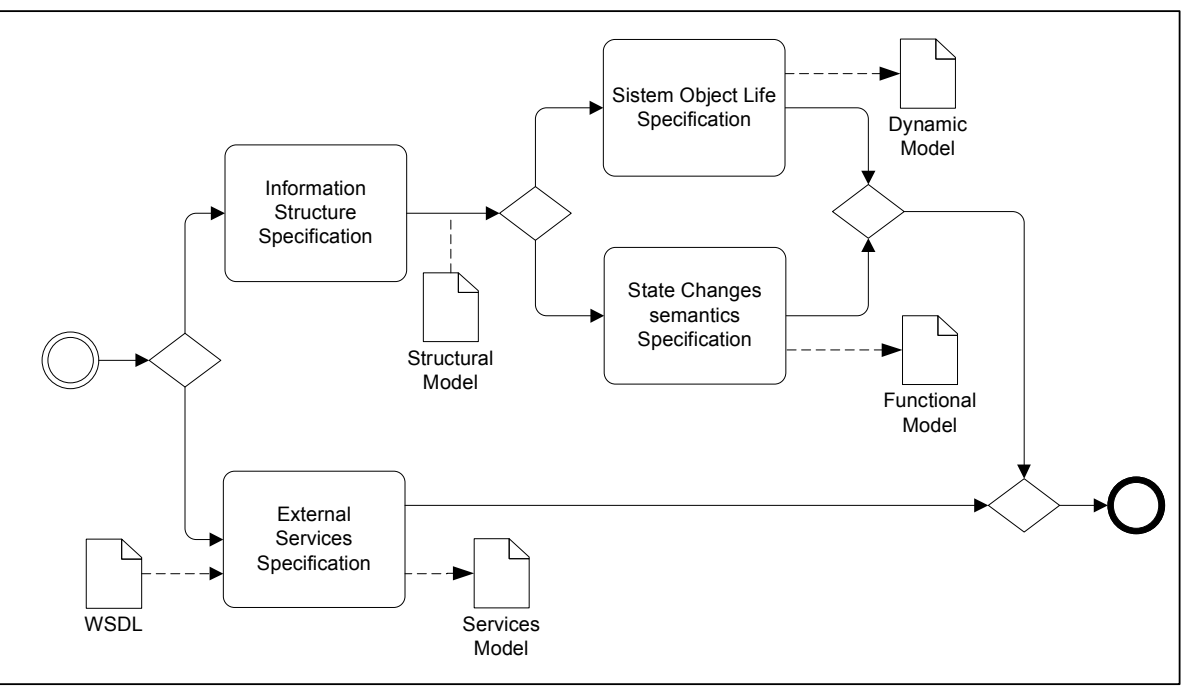

Figure 4.2 Expanded Sub-process System Specification

The following step in the process is still performed by the developer. At this point, the developer completes $^{20}$ the ABPs defined by the Analyst in order to generate, in a later step, an equivalent representation of the BPs but this time in terms of the WS-BPEL executable language. This is achieved by associating operations defined either in the Structural Model or in the Services Model to the tasks included in the ABPs. These associations will allow, in a later step of the process, to obtain Concrete $^{21}$ Business Processes (CBP).

20 This completion is only required when the ABP includes at least one task that has not been defined as manual, this is, a task whose execution is implemented in a system.

${ }^{21}$ Understanding "concrete" as an executable definition of the process. 
Once the system has been shaped in the previous mentioned models we can bring them into the Bizzy tool and use it for the generation of new artefacts ("Web Specification" task). This tool performs two different tasks which produce different artefacts from the specifications defined in the Structural, Services and Business Process Models. On the one hand, the tool generates the Navigational and Presentation models defined by the OOWS approach (see Figure 4.3). On the other hand, from the CBPs the tool generates the equivalent $\mathrm{BP}$ but this time as a WS-BPEL process ready to be deployed in a process engine.

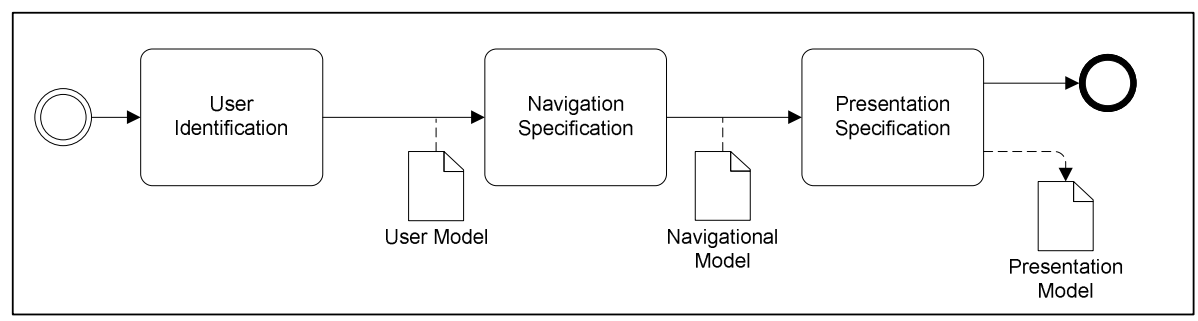

Figure 4.3 Expanded Sub-process Web Specification

\subsection{The Modeling Step}

This section presents the first step in the development process. This step involves representing the system requirements in terms of the models defined in the proposal.

\subsubsection{The OO-Method Modeling Step}

OO-Method provides a set of models that allow performing the conceptual modelling of Information Systems. This conceptual model is represented by two different aspects which are the 
system structure and its behaviour. These two aspects are gathered in the Structural, Dynamic and Functional models. In addition to these models, an additional model was introduced in (Torres et al., 2005; Quintero, 2008) to capture the functionality that is provided by external systems. All these models are explained in the following subsections.

\subsubsection{The Structural Model}

It defines the system structure (its classes, operations and attributes) and relationships between classes by means of a UML Class Diagram.

\subsubsection{The Dynamic Model}

It describes (1) the different valid object-life sequence for each class of the system using State Transitions Diagrams and (2) the communication between objects by means of Sequence Diagrams.

\subsubsection{The Functional Model}

It captures the semantics of the state changes to define service effects using a textual formal specification.

\subsubsection{The Services Model}

This model was introduced in the OOWS original proposal in order to cope with the integration of external functionality into the proposal. The objective of this model is bringing up external services (functionality provided by external partners) into the modelling level in order to manage them more easily. 


\subsubsection{The Business Process Model}

This model is used to specify the set of BPs that have to be supported by the system. Its specification is performed by means of the BPMN notation. The diagrams specified in this model are built in two steps. First of all, a general description of the processes is performed by the analyst. This description does not include details in terms of the system, but in terms of the domain. In a second step, these descriptions are taken and processed by the developer in order to include all the details that will convert them into process descriptions that can be transformed into executable processes. This model is explained in detail in chapter 5.

\subsubsection{The OOWS Method Modeling Step}

The OOWS Method extends the OO-Method approach in order to cope with the peculiarities introduced by Web applications. It defines three different models which gather three different aspects of Web applications, (1) the kind of users interacting with the system, (2) the way users can navigate through the system and (3) the way the system is presented to the users.

\subsubsection{The User Model}

It defines the kind of users that are going to interact with the web application. Moreover, it allows defining inheritance relationships between them. 


\subsubsection{The Navigational Model}

It captures the navigational structure of Web applications. This structure is defined as views over the system and different views are defined for each kind of user defined in the User Model. The extension to deal with the navigation modeling during $\mathrm{BP}$ execution is presented in chapter 6 .

\subsubsection{The Presentation Model}

It defines the presentation properties in which the information and functionality must be shown in the Web application. These properties are related to information paging, layout and ordering criteria and are applied to the elements that have been defined in the Navigational Model.

\subsection{The Code Generation Step}

In a second step in the development process, the domain specified in the models presented in the previous section is transformed into code artefacts that can be executed. This phase of the process includes two steps, one related with the generation of the code representing the service orchestration (BPs) and another representing the interface that will allow users involved in the process interacting with the BPs. In particular, this interface is going to be provided as a Web application.

\subsubsection{WS-BPEL Code Generation Step}

The generation process of an executable definition of the business process is made up of three steps as Figure 4.4 shows. 


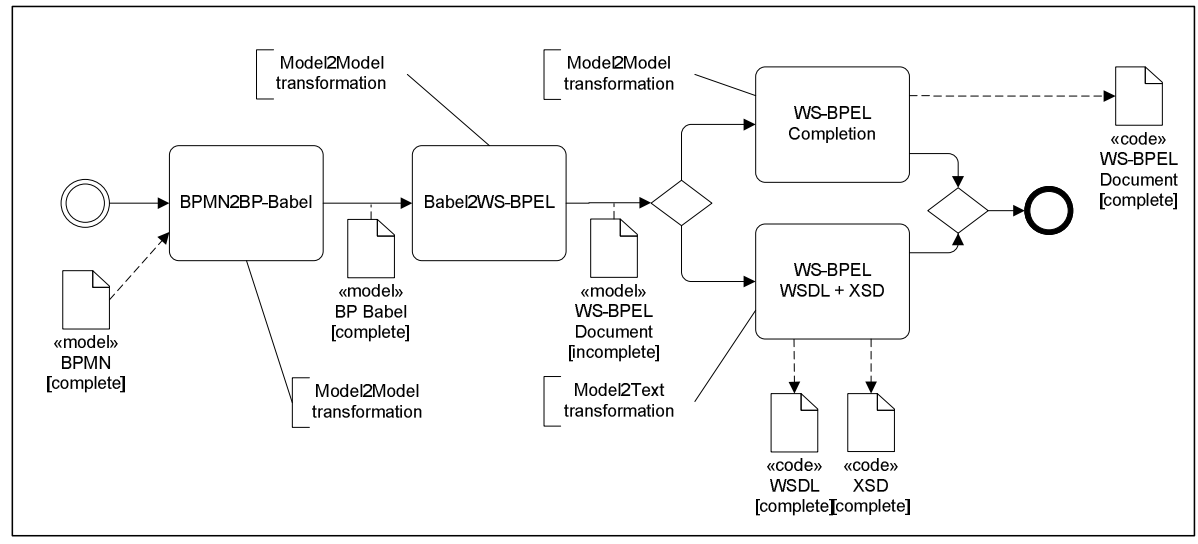

Figure 4.4 Expanded Sub-process WS-BPEL generation

First of all, the "BPMN2BP-Babel" task takes as input the abstract BPMN definition of the process and transforms it into the BP format accepted by the Babel tool. This step is performed by means of a model to model transformation (see section 9.2). Then, the "Babel2WS-BPEL" task transforms the BP definition into a partial WS-BPEL document (only the Activities section is generated being the Partner Link, Variables and Correlation Sets left). Then, the partial WS-BPEL document is completed in the "WS-BPEL completion" task. This task can complete the WSBPEL definition from the information gathered in the Structural and Services models. Finally, as a WS-BPEL process is also a web service, it is necessary to generate its interface which contains operations and data types. This is performed in the "WS-BPEL WSDL + XSD” task. 


\subsubsection{User Interface Code Generation Step}

The generation process to obtain the modelled system into a Web framework is performed in just one step (see Figure 4.5). In this step a set of model-to-text transformations are executed to obtain the modelled system in terms of a Web framework. Specifically, these transformations produce code according to the Tapestry Web framework. One of the main reasons to adopt this framework was the component-based model in which this framework bases. This allows marking a clear separation of different technologies used during the development of the Web application.

Therefore, the "Web application generation" task requires the OOWS model (including the OO-Method, the Services and the BP models) as input model.

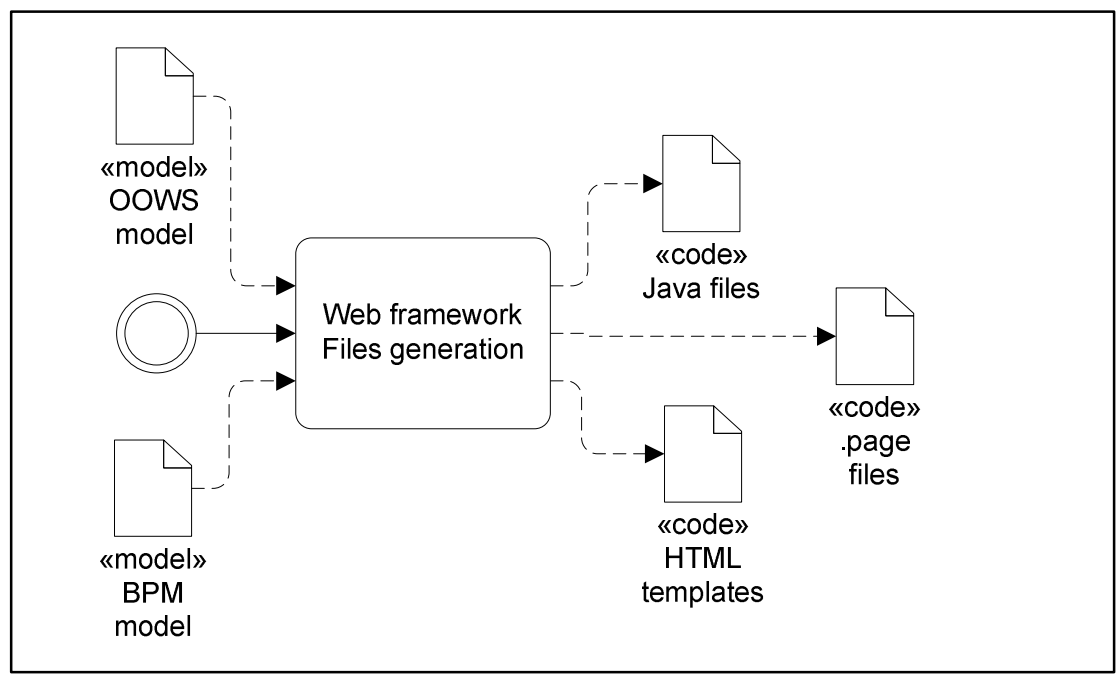

Figure 4.5 Expanded Sub-process Tapestry Web framework generation 
As Figure 4.5 shows, the "Web framework files generation" task produces three kinds of code files:

- The java files which define the java classes that implement the logic of the application. It corresponds to the Controller aspect from the MVC architecture in which this framework relies on.

- The HTML files which define the templates that correspond to the View aspect from the MVC architecture.

- The page files which are XML documents that include the declaration of the Tapestry components used in the HTML files. Although page files are optional, they contribute to obtain a more readable code.

A more detailed explanation about the Tapestry framework and the required files for building a Web application in this framework is presented in chapter 9 .

\subsection{Conclusions}

In this chapter we have presented the extended version of the OOWS Web Engineering method to deal with the modelling and generation process of BP-driven Web applications. The extension involves the introduction of a new step in the modelling process to specify the set of BP that the generated web application should provide support to. Moreover, the generation process has been also extended with a new step where BP definitions are transformed into executable processes. Chapter 9 includes the set of 
transformations that have been implemented to support the generation process. 



\section{Chapter 5}

\section{Business Process Model}

The Business Process Model (hereafther BPM) has been introduced into the OOWS method to deal with the specification of BPs at the modelling level. This model is based on the BPMN notation. However, the expressivity provided by this notation does not cover all the modelling requirements needed in the BPM. For this reason, we have defined some extensions to the original notation. These extensions allow us building BP specifications that are integrated with other models defined in the method and that can be automatically transformed into executable process definitions (in particular in WS-BPEL definitions). These extensions have been designed trying to modify the BPMN original notation as little as possible. In fact, an extended model is still a valid BPMN model (no structural or semantic changes have been performed).

The chapter has been organized as follows. Section 5.2 present in detail the modelling limitations found in the original BPMN notation. Section 5.3 presents a $\mathrm{BP}$ example that highlights the limitations previously presented. Then, by using the example, 
section 5.4 details the extensions defined to deal with the limitations. Finally, section 5.5 provides some conclusions.

\subsection{Business Process Model based on the BPMN notation}

The BPM has been introduced in the OOWS original proposal to provide developers with modelling mechanisms for the specification of Business Processes (BP). This model allows describing system functional requirements that are provided in terms of a well-defined process. The type of information that is represented in a BPM includes the set of tasks that form the process and how these tasks are distributed among different participants.

As we have stated in chapter 2, the BPM is specified by means of the BPMN notation. This notation defines three basic types of submodels which are (1) private (internal) business processes, (2) abstract (public) processes and (3) collaboration (global) processes. In this work we combine two of these basic sub-models to model $\mathrm{BP}$ requirements. On the one hand, private business processes are used to define internal processes of a specific organization (for which we are developing the system). On the other hand, abstract processes are used to represent interactions between private business processes and external participants.

The BPM is represented as a single Business Process Diagram (hereafter BPD) that includes multiple private and abstract 
processes where the activities that are common to several processes are associated. In the last step of the development process, by the application of model-to-model transformations, each private business process is mapped to one WS-BPEL document. Moreover, this document also includes the specification of the interaction between the private process and the external partners defined in the abstract processes. Specifically, this usage of the BPMN notation corresponds with one of the types of BPD covered by the notation (OMG, 2006), which corresponds to the "Detailed private business process with interactions to one or more external entities (or "Black Box" processes)" diagram type.

The information that has to be gathered in the BPM corresponds to (1) the systems (external and internal) that are involved in the $\mathrm{BP},(2)$ the activities that conform the internal (private BP) system, (3) the participants (within the private BP) that are responsible of performing these activities, (4) the conditions that control the BP flow and (5) the interaction that occurs between the private $\mathrm{BP}$ and the external partner(s). To represent all this information in a BPD, the BPMN notation provides us with different elements that allow representing the information that need to be specified in the BPM. These elements have already been presented in chapter 2 but the main groups are briefly presented now.

- Swimlanes: The elements included in this group allow grouping primary modeling elements. These elements are Pools, and Lanes and are defined in the specification as follows: 
- Artifacts: The elements included in this group are used to provide additional information about the Process. Nowadays, the BPMN specification includes Data Object, Group and Annotation, however, this set is susceptible to grow and standardize either for general use or for vertical markets. A common characteristic of the elements included in this group is that none of them have a direct effect on the Sequence or Message Flow of the Process.

- Flow Objects: The elements included in this group define the behaviour of a BP. These elements are Events, Activities and Gateways.

- Connecting Objects: The elements included in this group allow connecting Flow Objects to each other. The specification distinguishes three kind of connecting objects which are Sequence Flow, Message Flow and Association.

\subsection{BPMN limitations to Support the BPM}

The four groups of elements roughly presented in the previous section gather the set of graphical elements used in the BPD. However, in order to produce executable definitions equivalent to those modelled in the BPMN notation it is necessary to provide detailed information to some of the elements conforming these BPs. Within the set of BPMN graphical objects we find almost all the necessary elements for defining the kind of processes we are interested in. Nevertheless, the notation does not allow us defining: 
1. The scenarios where human participants behave as individuals or as members of a particular group.

2. The functionality that is going to support each task/activity included in the BP definition. Note however that this do not applies to tasks defined as manual (tasks that do not change the state of the Information System and that are completely carried out by users).

3. Dependencies between different lanes within the same pool. In some cases process participants are related each other and this link have to be explicitly defined in the process.

To deal with these limitations we have defined extensions to the original BPMN notation. Specifically, the proposed extensions have been defined over the BPMN Metamodel included in the BPMN modeller ${ }^{22}$ from the STP (SOA Tools Platform) project. The modeller main goal is to provide a graphical notation that allows defining processes. This tool has been developed based on GMF (Graphical Modeling Framework) and reuses and extends the GEF (Graphical Editing Framework) and the EMF (Eclipse Modeling Framework) projects.

\subsection{PhD Thesis Delivery Example}

The $\mathrm{PhD}$ Thesis Delivery BP depicted in Figure 5.1 represents the procedure defined by a University Department to present a PhD Thesis work. This BP is fully performed by participants of the same organization (all of them are defined in different lanes

$22 \mathrm{http}: / / \mathrm{www} . e c l i p s e . o r g / \mathrm{stp} / \mathrm{bpmn} /$ 
within the same pool). However, the behaviour of the participants is different depending on the role. For instance, the Member role (Member lane in Figure 5.1) is supposed to behave as an individual. This means that the same person is going to perform all the tasks included within this lane. Therefore, the person delivering the Thesis draft (first activity of the BP) has also to fix up the recommended corrections, fill in the handout request, and all the remainder tasks that have been specified within the Member lane. Nevertheless, this is not the case for other lanes such as the Member.supervisor, Secretary and Department Commission lanes. In these cases, any person belonging to the any of these groups can perform any of the tasks that have been defined within their lane.

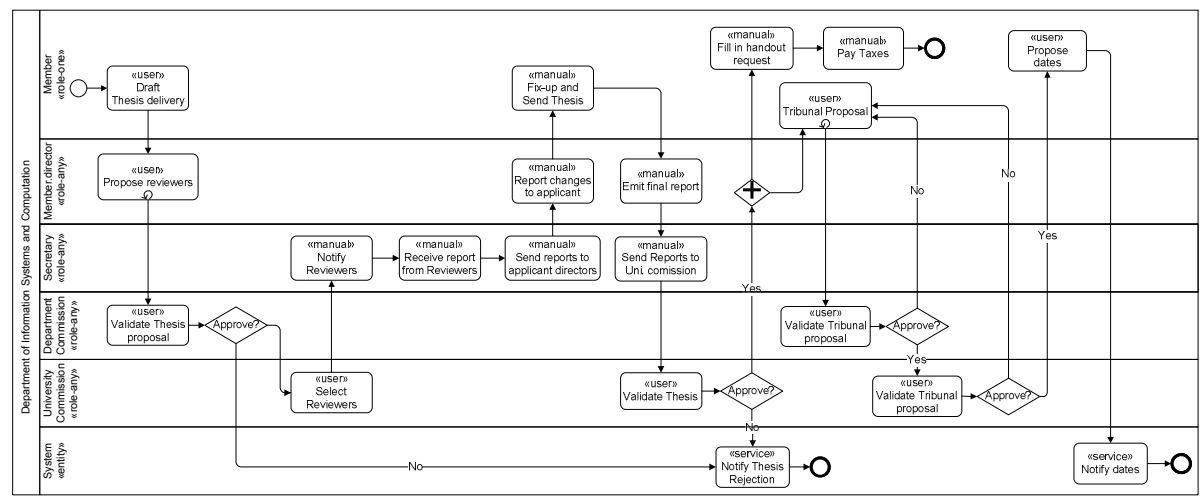

Figure 5.1 PhD Thesis Delivery BP

In addition, process tasks can be defined of different types. Some of them are fully performed by humans (i.e. "Notify Reviewers"), others are performed by humans but being assisted by the system (i.e. "Draft Thesis Delivery") and others are performed completely by the system (i.e. "Notify Dates"). From these types, only process 
tasks included in the last two types are associated with some system functionality. Figure 5.2 depicts (1) an enlarged excerpt of the PhD Thesis Delivery BP and (2) an excerpt of the Structural Model built for this example.
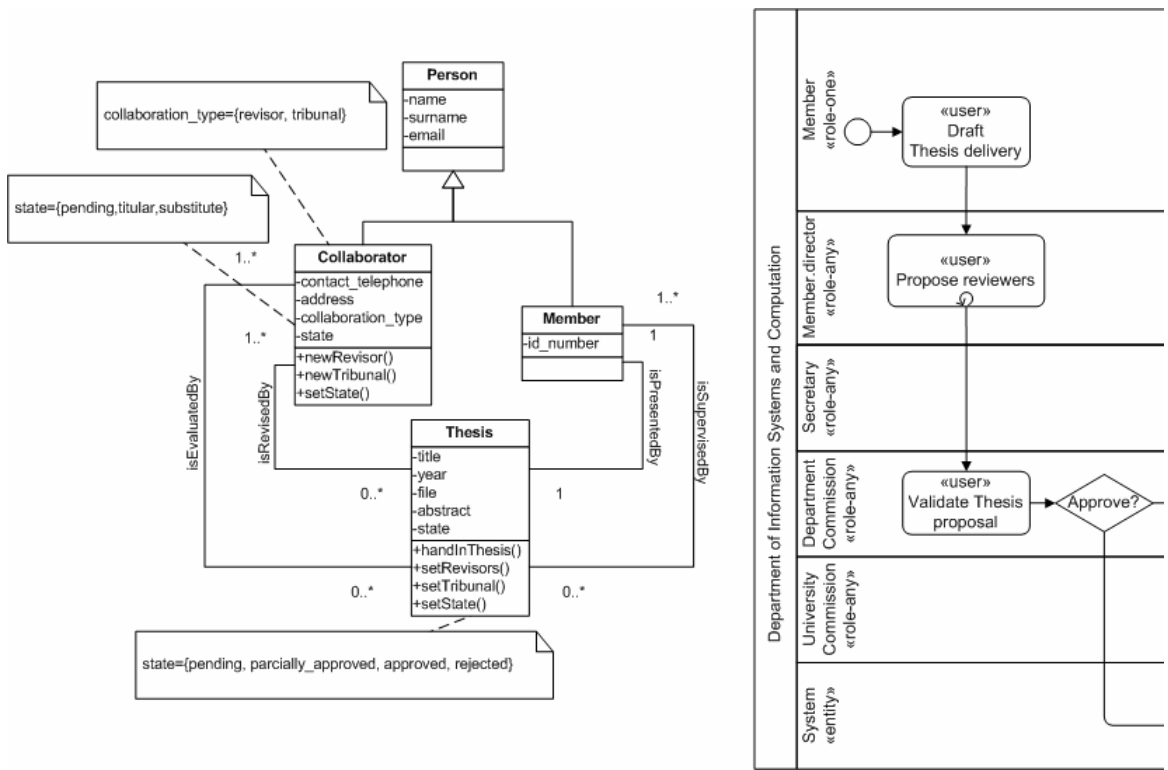

Figure 5.2 Excerpts of the Structural Model and Business Process

Diagram

Another particularity of the $\mathrm{PhD}$ Thesis Delivery BP is that the specific supervisors involved in the process depend on the member delivering the thesis. On the contrary, other participants such as the Secretary Staff, the Department and the University Commissions are always the same independently of the specific user delivering the thesis. 


\subsection{BPMN Extensions}

This section is divided into three sub-sections which are dedicated to present the solutions designed to deal with the limitations presented previously.

The extensions have been organized in a new metamodel called bpmnxModel which extends some of the concepts defined in the original BPMN metamodel. Figure 5.3 shows how the original BPMN metamodel and the extension have been organized in different packages.

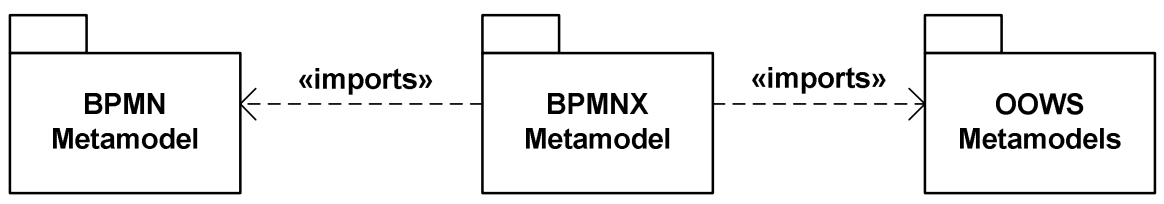

Figure 5.3 Metamodel Architecture

The extension defined over the BPMN metamodel (see bpmnx Metamodel package in Figure 5.3) has been implemented by creating a new EMF model that references (1) the elements that are going to be extended from the original specification (see import relationship between the bpmn Metamodel and the bpmnx Metamodel packages) and (2) the functionality that has been defined in the Structural and Services models (models that are included in the oows Metamodel package).

The details of the defined relationships between the bpmnx package and the bpmn and the oows packages are presented in Figure 5.4. As this figure shows, the XLane and the XActivity 
classes extend the Lane and Activity concepts defined in the BPMN original metamodel.

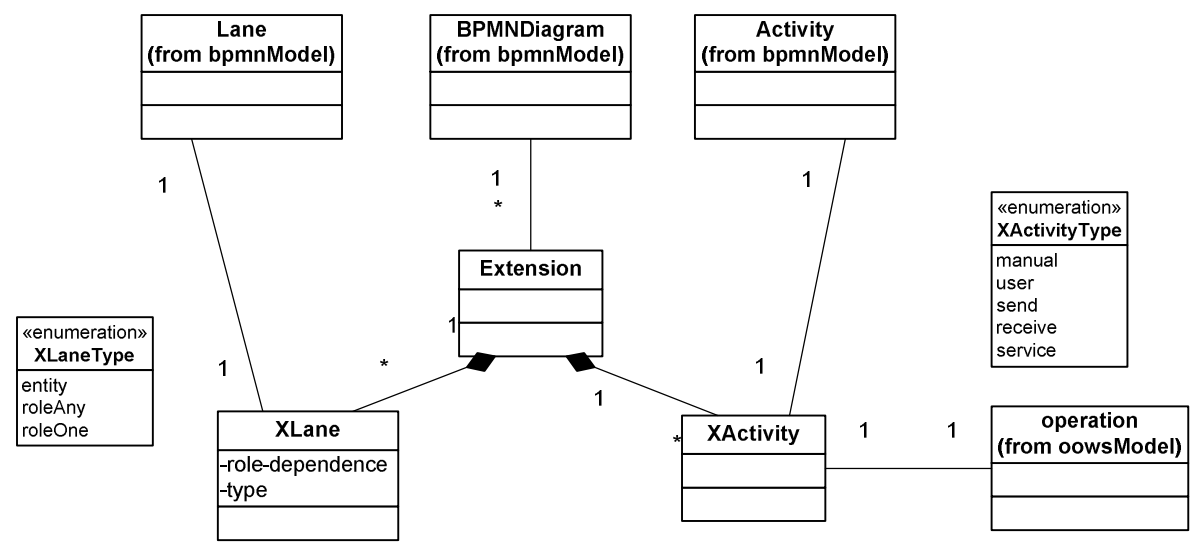

Figure 5.4 Extension Defined to the BPMN Metamodel

The following subsections explain each extension. This explanation is carried out in two steps. First, a concrete syntax based on the example presented in section 5.3 is provided. Then, the abstract syntax of the introduced elements (concepts and relationships) is performed.

\subsubsection{Differentiating Human Participant Behaviour}

Concrete Syntax: In the PhD Thesis Delivery BP, the Member lane has been marked with the "role-one" value. On the contrary, the remainder lanes have been marked with the "role-any" value.

Abstract Syntax: In order to differentiate the behaviour of a process role we have extended the Lane element with a new attribute, the type attribute. The values accepted by this new attribute are role-one and role-any. 
- The role-one value is used when the user behaves as an individual. Therefore, the human being performing the first task of the lane has to be the same for the rest of tasks defined within the same lane.

- The role-any value is used when the user behaves as a member of a group. In this case, any human being belonging to the group specified in the lane can perform any of the tasks included in it.

Specifically, this extension has been implemented by adding the type attribute to a new class, the XLane class, which extends from the Lane class defined in the original BPMN notation. This attribute has been designed as follows:

- The type attribute has been introduced to specify the behaviour of the role involved in the current lane. It can take one of the three different values: entity (used when the participant is not a human being), roleOne (used when the participant is a human being that participates in the current process as an individual) or roleAny (used when the participant is a human being that participates in the current process as a member of a group). These values are defined in the XlaneType enumeration.

\subsubsection{Defining the Functionality of each Process Task}

Concrete Syntax: In the $\mathrm{PhD}$ Thesis Delivery BP, tasks defined as "user" and "service" has to be linked to operations defined in the Structural Model. For instance, the first activity "Draf Thesis delivery", which is defined as "user", is associated with the 
"handInThesis()" operation defined in the Thesis class (see Figure 5.2). In this activity, the user inputs the required data (specifically the data required by the operation) and the system executes the operation which creates (1) an instance of the process and (2) an instance of the thesis class. Similarly, the "Propose reviewers", "Validate Thesis proposal" and "Select Reviewers" activities are associated with the "Collaborator.newRevisor()", "Thesis.setState()" and "Collaborator.setState()" operations respectively.

Abstract Syntax: A necessary step to obtain an executable definition of the process is specifying the functionality that is going to be performed in each task. For this purpose, we have extended the Task element with a new attribute, the operation attribute, which associates tasks with functionality that has been defined in the Structural or in the Services Model. On the one hand, the Structural Model defines functionality that is provided by the local system, specifically by the classes defined in it. On the other hand, the Services Model defines functionality that is provided by external systems, specifically by the imported Web services. However, this extension only applies to tasks defined as Service, Receive, Send and User (these are some of the types in which a task process can be defined) in the BPMN notation. Process tasks defined as Script or Manual are not susceptible of this extension. 
Specifically, this extension has been implemented by adding the operation attribute to the XLane class. This attribute has been designed as follows:

- The operation attribute is used to reference an operation defined either in the Structural model or in the Services model.

\subsubsection{Lane Dependences}

Concrete Syntax: In the PhD Thesis Delivery BP, the "dynamic" lane has been labelled as "Member.supervisor" indicating the dependent lane (Member) and the relationship between these two lanes (supervisor). In addition, the role-dependence attribute has set to the navigation expression: Thesis.isSupervisedBy which allows identifying the users that can perform the tasks included in the Member.supervisor lane. With regard to the order in which tasks have to be performed, Figure 5.1 shows that the "Draft Thesis delivery" task in charge of creating an object of the Thesis class is performed previously to the "Propose Reviewers" task which can be performed by any of the supervisors associated to the Thesis previously delivered.

Abstract Syntax: In order to define dependencies between different roles included in the $\mathrm{BP}$ we have extended the Lane element with a new attribute, the role-dependence attribute. This attribute is valued with an expression linked to the Structural Model that allows identifying the users that form this "dynamic" role. This expression corresponds to navigation over associations defined in the Structural Model. In addition, this dependency 
forces to perform first the activities that create or selects the object involved in the navigation expression. Finally, to identify more easily dependencies between lanes we label "dynamic" lanes as follows: [dependent_lane].[relationship].

Specifically, this extension has been implemented by adding the role-dependence attribute to the XLane class. This attribute has been designed as follows:

- The role-dependence attribute has been introduced to specify the dependence of the current lane with another lane defined within the same pool. This relationship has to be expressed in terms of the relationship defined in the Structural model between these users. The notation used to define this link is related with the relationship defined in the Structural model.

\subsection{Conclusions}

Business Process models play a very important role in the development process of enterprise software systems. Although depending on the area in which enterprises develop their functionality, it is quite likely that some of the requirements of the system were given as a process. Then, once these process specifications are given, these can serve as two purposes. On the one hand, it allow business analysts to define clearly (as these specifications are usually performed in languages or notations targeted to this kind of users) the business process that systems should implement. On the other hand, these specifications can 
also be used as input in the development process to derive new artefacts (executable process definitions and graphical user interfaces) related to it.

This chapter has presented a set of extensions defined over the original BPMN notation. The main reason to define these extensions is integrating $\mathrm{BP}$ specifications into the OOWS approach and detailing these specifications to transform them into an executable language.

Finally, the availability of tools developed under the umbrella of the Eclipse project (and in particular the use of the EMF project), as it is the case of the BPMN modeller, has facilitated the development of the proposed extension.

Summing up, the introduction of the Business Process Model with the characteristics that have been explained in this chapter endows the method to overcome seamlessly the development of Web applications that are highly driven by Business Processes. 


\section{Chapter 6}

\section{Navigational Model Extension}

This chapter presents the extensions that have been introduced into the OOWS Navigational Model to deal with the integration of Business Processes (BP). These extensions have been designed taking into account the characteristics of the kind of BPs (shortrunning and long-running BPs) we are dealing with (see chapter 2 ). The main reason to introduce new primitives is to be consistent with the separation of concerns in which the OOWS method has been conceived since its inception.

The chapter has been structured as follows. Section 6.1 provides an introduction to this chapter. In this section, a motivation for the introduction of these primitives is provided. Then, the extension is presented through the following three sections. First, in section 6.2 the extension is presented intuitively by means of two case studies. Second, a more formal description of these primitives is presented in section 6.3. Third, an overview of the Navigational metamodel is presented in section 6.4. Finally, section 6.5 presents the conclusions of this chapter. 


\subsection{Introduction}

The interaction that takes place between the user and the system during the execution of a $\mathrm{BP}$ is different from the one occurring during content navigation. On the one hand, when the user navigates through the Web application (content navigation), she is the one deciding the path (link) to follow. On the other hand, when the user is executing a $\mathrm{BP}$ (BP navigation), is the system that drives the user through the application.

The set of new primitives presented in this chapter allow capturing, relating and manipulating the different aspects that are introduced by the execution of BPs which are:

- The required data and functionality to perform BP activities. The required functionality is specified by means of the class-view, service-data-view and service-functionalview primitives. These primitives are views over classes and services defined in the Structural and Services Models respectively and define the data and functionality that is associated to a specific BP activity.

- The access point that allow users reaching BPS. This access points allow users (1) starting new instances of a specific BP and (2) taking up again a started BP instance that was previously suspended. This is specified by means of the Process Context and the Process Link primitive.

- Complementary information that help users completing BP tasks. By providing users with information related to the BP activities being performed we (1) help them in 
completing the activity and (2) avoid them switching from $\mathrm{BP}$ to content navigation to reach that information. This information is specified by means of the Complementary$A I U$ primitive.

- Mechanisms to filter the amount of data shown to the user. To avoid overloading GUIs with too much information it is necessary to specify the filters that are going to be applied to the retrieved instances. This filtration is specified by means of the Index primitive.

- Other primitives have been introduced to organize data and functionality according to the requirements of each $\mathrm{BP}$ activity. For this purpose, primitives such as Activity Containers, Main-AIU and Human-AIU have been defined.

\subsection{Introducing the New Navigational Primitives}

Before detailing each primitive, this section introduces them graphically. To cover all the primitives, two different examples are used. Both examples are presented in two steps. First, the Navigational map for a specific type of user is presented (Authoring-in-the-large). Second, a detailed view of the Process Context included in the Navigational Map is presented (Authoring-in-the-small).

The first example is related to the "Book Purchase Request" BP. Within the categorization defined in chapter 2, this process corresponds to a long-running BP. In this case, Figure 6.1 presents the Navigational map for the Secretary User type. 


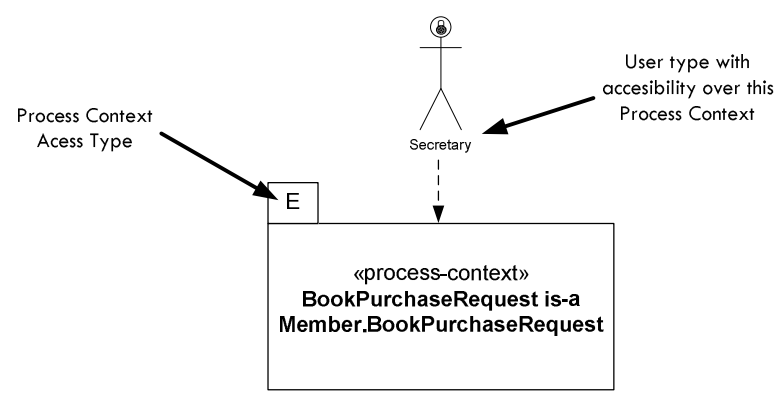

Figure 6.1 Navigational Map for the Secretary User type (Authoring-inthe-large)

Figure 6.2 shows the detailed view of the "BookPurchaseRequest" Process Context depicted in Figure 6.1. This figure includes most of the primitives included in the extension.

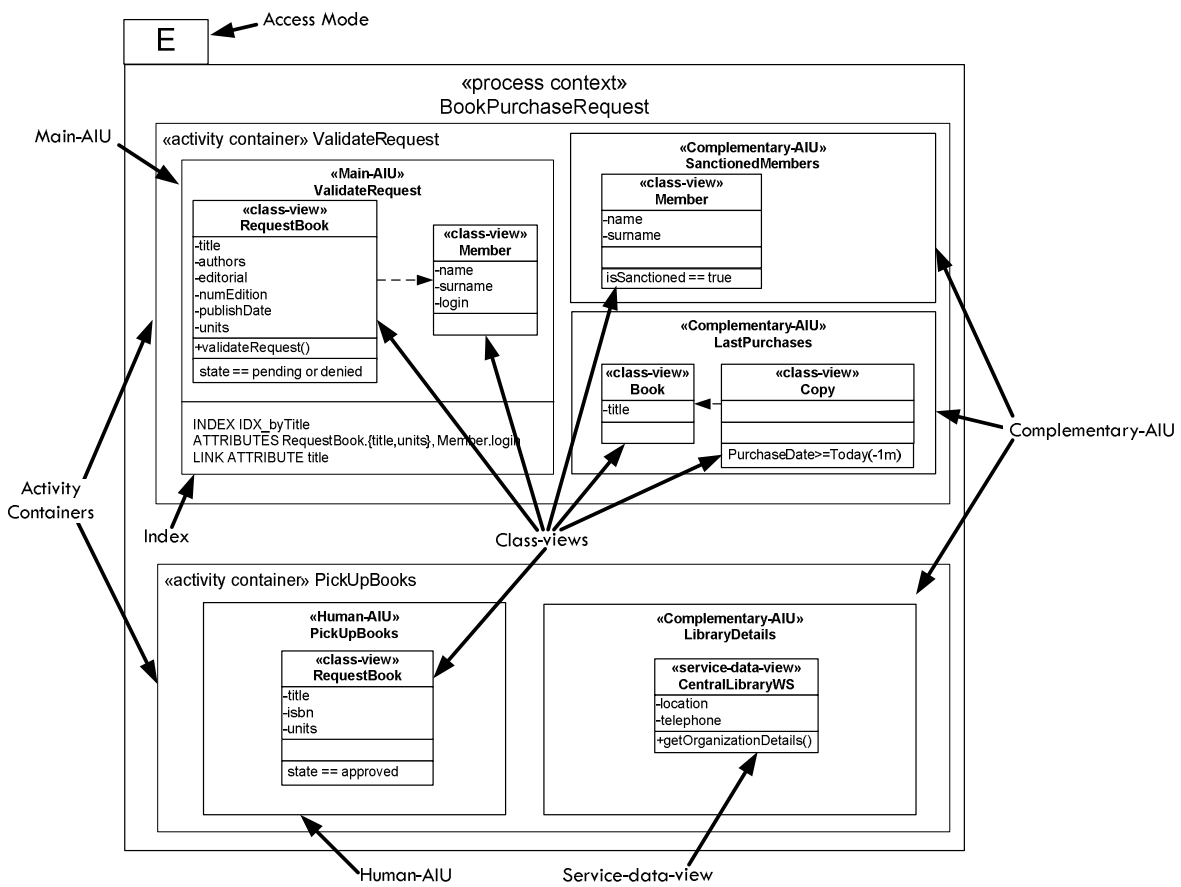

Figure 6.2 Book Purchase Request Process Context (Authoring-in-thesmall) 
The second example is related to the "Checkout" BP. Within the categorization defined in chapter 2 , this process corresponds to a short-running BP.

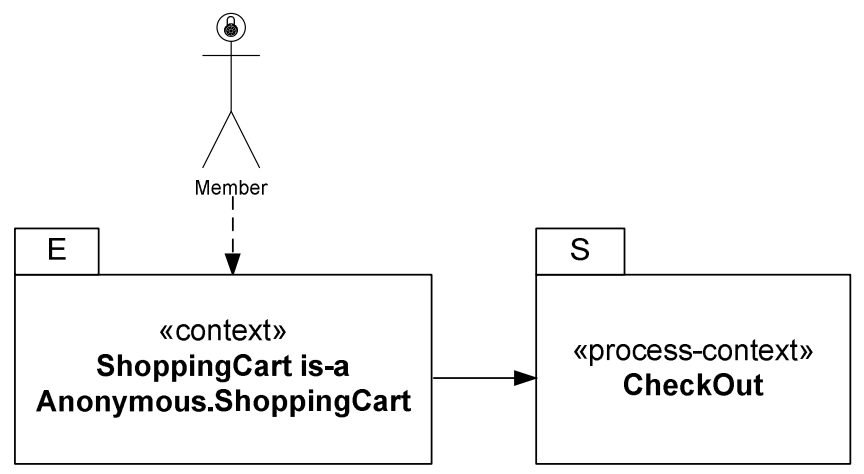

Figure 6.3 Navigational Map for the Member User type (Authoring-inthe-large)

In this case, Figure 6.3 presents the Navigational map defined for the Secretary User type.

Finally, Figure 6.4 shows the detailed view of the "Checkout" Process Context depicted in Figure 6.3.

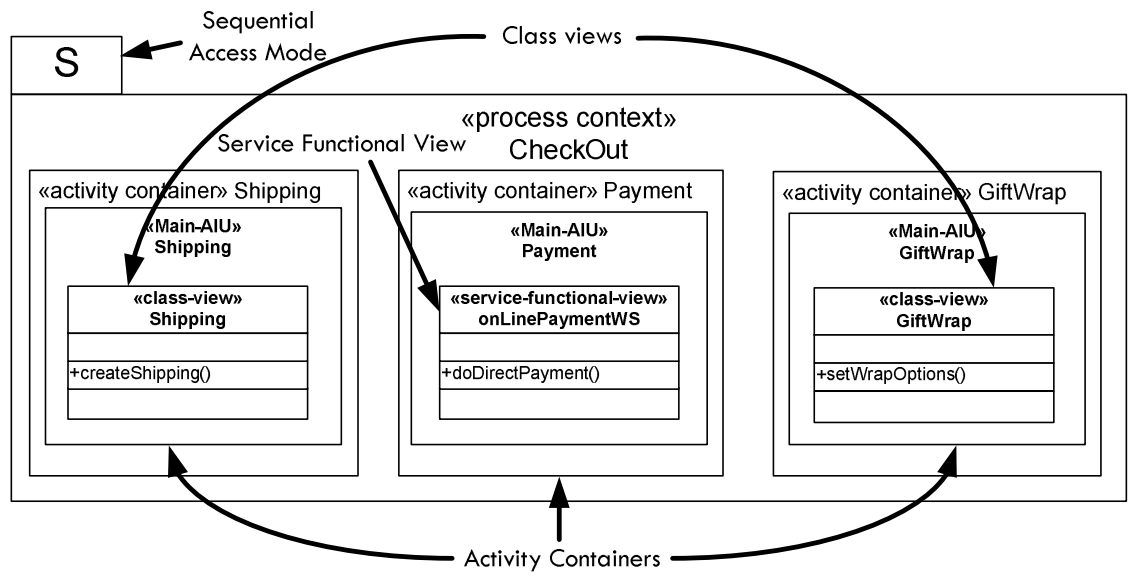

Figure 6.4 Check Out Process Context (Authoring-in-the-small) 
Along this chapter, these figures are referenced to explain the primitives included in it.

\subsection{Navigational Primitives to Support BP execution}

The extension defined over the Navigational Model defines ten primitives, which include new ones and others that have been adapted to the context of BPs. All these primitives represent an abstraction of the elements included in the GUI used for completing BPs. The new primitives are Process Context, Activity Container, Main-AIU, Human-AIU, Complementary-AIU, ServiceData-view, Service-Functional-view and Process-link. The adapted ones are Index and Class-view. Each primitive is going to be presented according to this schema: First, the problem that motivates the definition of the current primitive is presented. Second, the concrete syntax of the primitive is presented. This syntax is described in a diagrammatic form and defines how the abstract syntax (which is presented next) is presented to the end user. Finally, the abstract syntax of the primitive is presented. In this case, the concepts associated to the primitive and their relationships to other primitives (even from another metamodel) are described.

\subsubsection{Process Context}

Problem: We need to specify which GUIs are associated to a particular BP. In addition, it is necessary to specify how a BP is 
reached, this is, which navigational paths have to be followed to reach a specific $\mathrm{BP}$.

Concrete Syntax: Figure 6.2 and Figure 6.4 illustrate a detailed view (authoring-in-the-small) of the "Book Purchase Request" and "Checkout" BPs respectively. With regard to the "Book Purchase Request" BP, the process context has been defined as "Exploration" context and has been associated to the Secretary User type. This means that users belonging to the Secretary User type can reach this Process Context from any place of the Web application. In addition, this Process Context has been defined from the "Book Purchase Request" Process Context specified for the Member User type (this is possible because in the User Model we have defined an inheritance relationship between the Member and the Secretary User types). This inheritance mechanism allows redefining contexts based on the user responsibilities.

The strategy proposed to allow users reaching long-running BPs defined as "Exploration" context, is to gather these BPs into a specific section called "Processes". Figure 6.5 illustrates the Web page corresponding to this "Processes" section. In addition, this section includes the "my TODO List" section which provides the logged users a shortcut to access their pending tasks. 


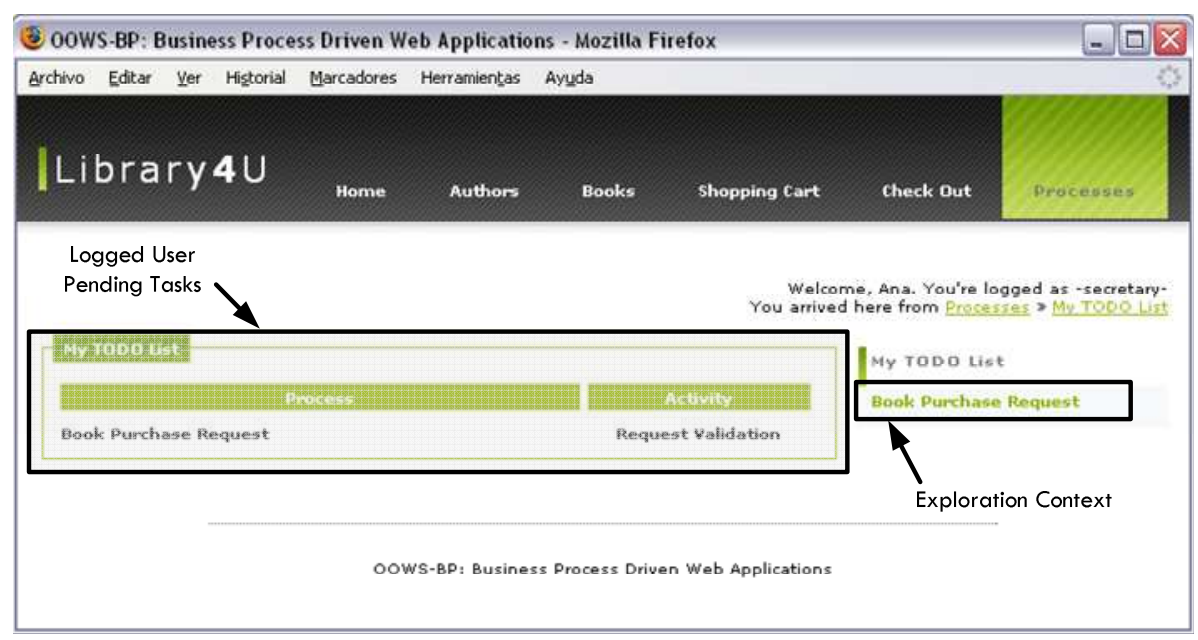

Figure 6.5 Web page corresponding to the "Processes" Section

With regard to the "Checkout" $\mathrm{BP}$ example, this has been defined as "Sequence" context and has been associated to the Member User type. This means that users belonging to the Member User type can reach this Process Context throughout the "Shopping Cart" Navigational Context. Figure 6.6 illustrates the implementation of the "Shopping Cart" Navigational Context. In this Web page, a link to start the "Check Out" BP is provided to the user. 


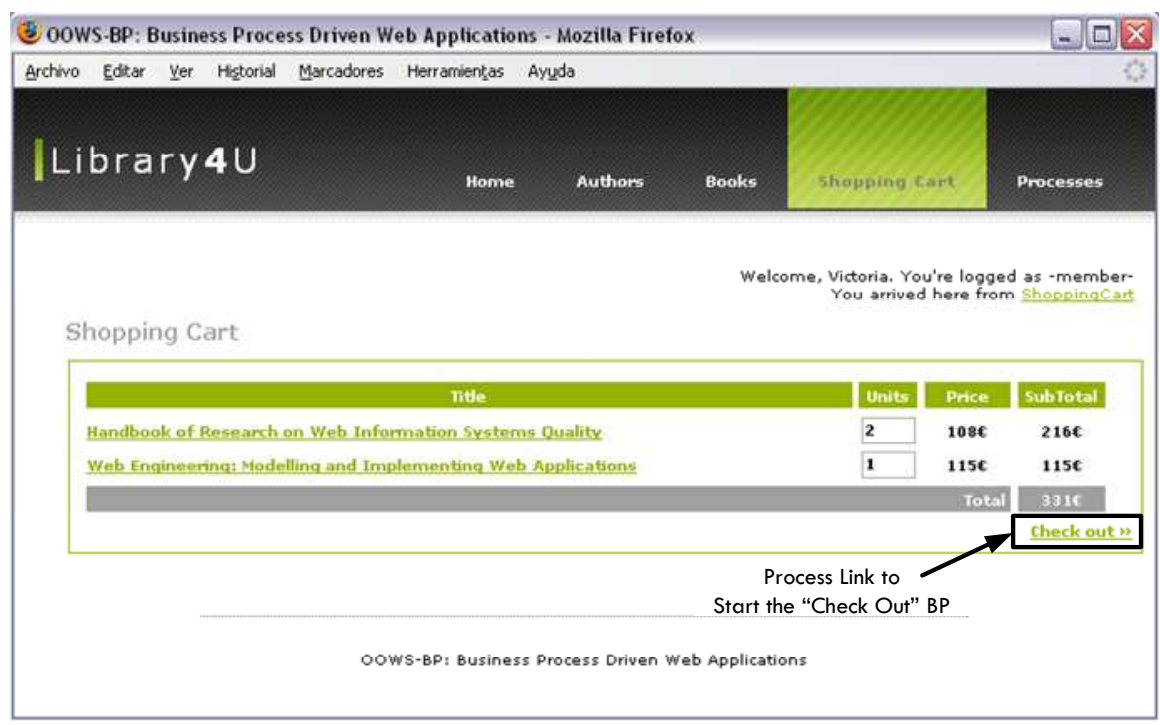

Figure 6.6 Web page corresponding to the "Shopping Cart" Navigational Context

A generic diagram of this primitive is shown in Figure 6.7. As this figure shows, a detailed view of a Process Context is depicted graphically as a UML package stereotyped with the keyword «process-context» (see the white part of Figure 6.7) and containing the name that corresponds to the referred process.

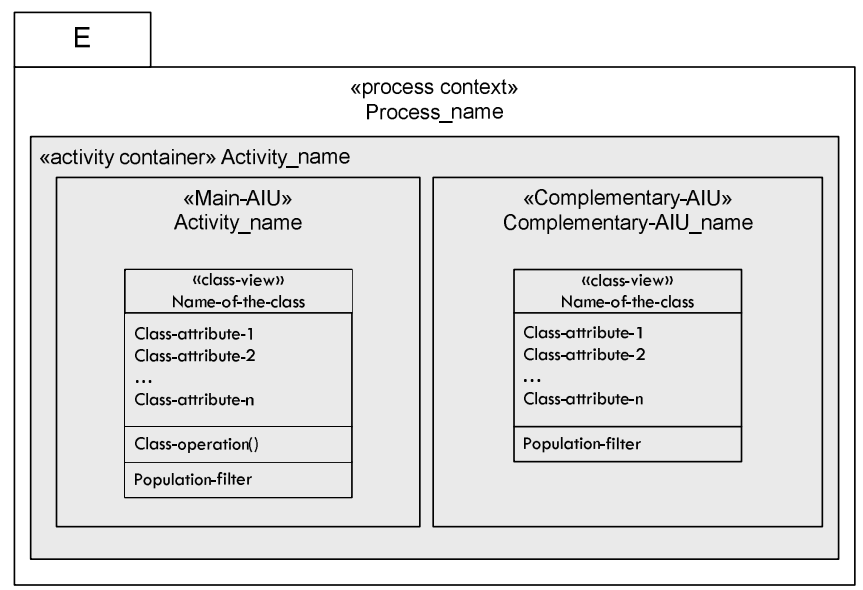

Figure 6.7 Process-Context primitive 
Abstract Syntax: The Process Context primitive abstracts the concept of a BP at the Navigational model. For each user defined in the User Model participating in any process we include a Process Context in her corresponding Navigational map.

The Process Context primitive has been designed as a specialized class of the NavigationalNode primitive defined originally in OOWS (see Figure 6.8). Similarly to the NavigationalContext class (context defined for content navigation), the ProcessContext class inherits the attributes defined in the NavigationalNode class which represent (1) the name that is going to be displayed to the user (alias attribute), (2) the identifier for internal purposes (id attribute) and (3) the reachability type for the context (reachability attribute).

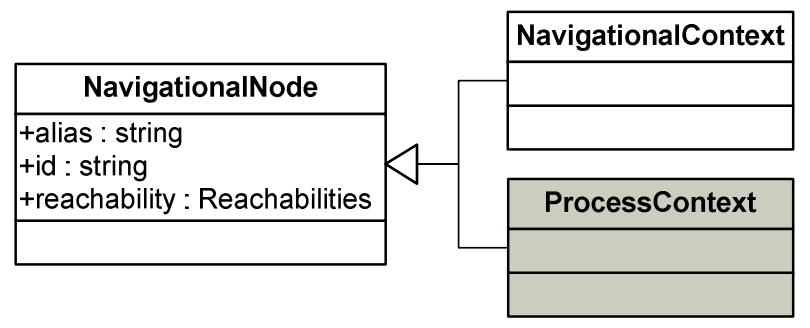

\begin{tabular}{|l|}
\hline $\begin{array}{l}\text { «enumeration» } \\
\text { Reachabilities }\end{array}$ \\
\hline $\begin{array}{l}\text { +Exploration }=0 \\
+ \text { Sequence }=1\end{array}$ \\
\hline
\end{tabular}

Figure 6.8 Metamodel Excerpt including the Process Context primitive

The reachability type defines the way users can access a specific Process Context and the values accepted by this type are:

- Exploration: This reachability type represents contexts that are always accessible from any part of the application. For long-running BPs, all the Process Contexts defined 
according this type are gathered in an always accessible section called "Processes".

- Sequence: This reachability type represents contexts that are only accessible by means of a predefined path. This sort of contexts is reached through a set of chained Navigational Contexts whose last context in the chain includes a link to the Process Context (see Process-link in 6.3.10 subsection).

\subsubsection{Activity Container}

Problem: We need to specify the data and functionality that is associated to a specific BP activity.

Concrete Syntax: Figure 6.2 and Figure 6.4 define two Process Contexts which include different Activity Containers, specifically, one per each activity defined in the BP involving human participation. Each Activity Container gathers all the data and functionality that are necessary to complete the associated BP activity.

Figure 6.9 illustrates how the "Validate Request" Activity Container included in the "Book Purchase Request" BP (see Figure $6.2)$ is presented at the implementation level. 


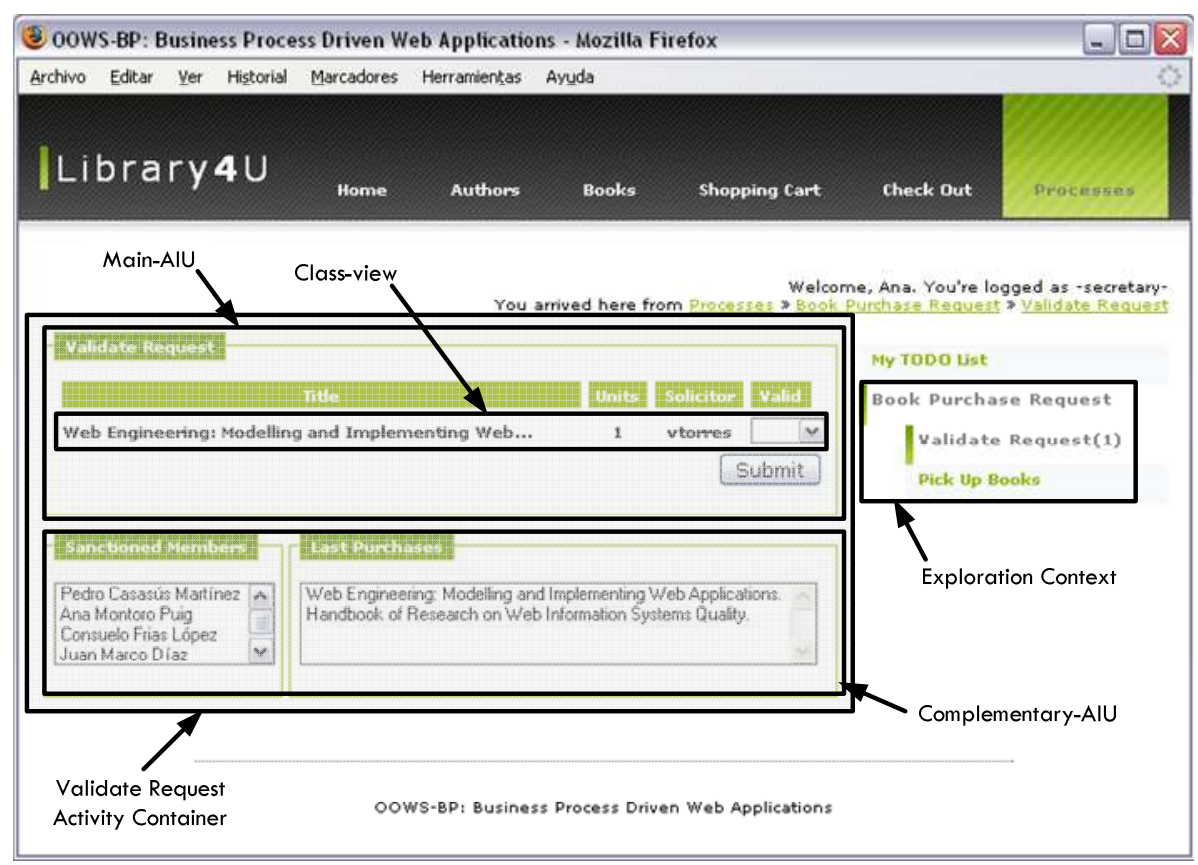

Figure 6.9 Web page corresponding to the "Validate Request" Activity Container

A generic diagram of this primitive is shown in Figure 6.10. As this figure shows, an Activity Container is depicted graphically as a white square (see the white part of Figure 6.10) contained within a specific Process Context. In addition, it contains the keyword «activity-container» which is followed by the name of the activity. 


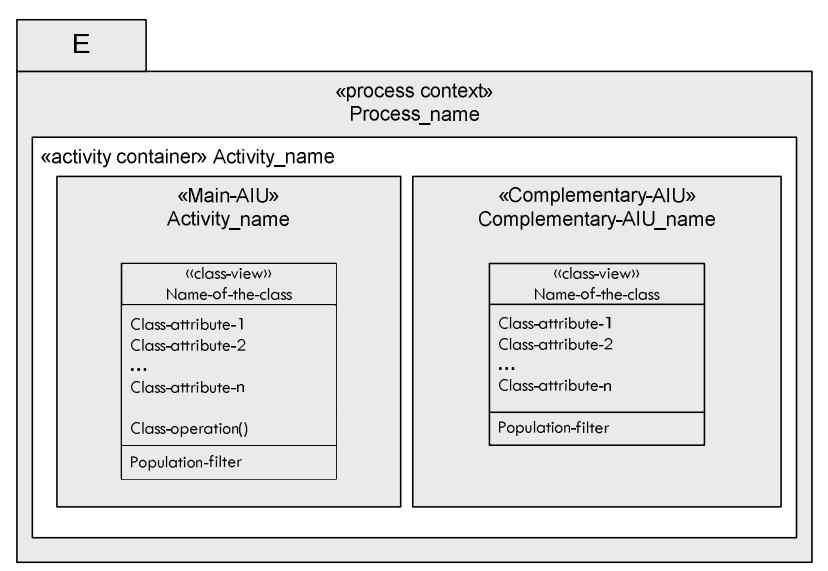

Figure 6.10 Activity-Container primitive

Abstract Syntax: The Activity Container primitive abstracts the concept of a BP activity at the Navigational model. It is contained within the Process Context primitive previously presented. Each activity modelled in the Business Process Model (BPM) and defined as «user» or «manual» will have a corresponding Activity Container in the Navigational Model. This primitive gathers the required data and functionality that has to be provided in the GUI to the user to complete the corresponding BP activity. An Activity Container is made up of different Abstract Information Units ${ }^{23}$ (AIU). An Activity Container must include either a Main-AIU or Human-AIU and zero or many Complementary-AIUS (these primitives are explained in sections 6.3.3, 6.3.4 and 6.3.5 respectively). This constraint is defined in the metamodel by

${ }^{23} \mathrm{An}$ AIU is defined in the original proposal as a "primitive representing the requirement of retrieving a chunk of related information". This primitive allows the coexistence of different information units in the same web page (requirement that is usually demanded in Web portals). 
means of a multiplicity restriction defined between the Activity Container and the abstract BaseAIU classes (the BaseAIU generalizes the Main-AIU and the Human-AIU classes). Activity Containers define two attributes which are initial and createProcessInstance. On the one hand, the initial attribute is valued to true when the Activity Container corresponds to the first activity of the process requiring interaction with a human being. Within the same Process Context, no more than one Activity Container can be defined as initial. This means that the BP is started by the user associated to the Process Context. On the contrary, when no Activity Containers are defined as initial, the BP is started by a system. This constraint is expressed in the OCL language as follows:

Context ProcessContext

inv: (self.activities.initial->select $(\mathrm{a} \mid \mathrm{a}=$ true $))->\operatorname{size}()=1$

On the other hand, the createProcessInstance attribute is valued to true when the Activity Container represents the first activity of the process (independently of its type). Moreover, only Activity Containers whose initial attribute is set to true can value their createProcessInstance attribute to true. The reason is that only the initial activities can create an instance of the associated BP. This constraint is expressed in the OCL language as follows:

\section{Context ProcessContext}

inv: (self.activities.initial->select(a|a = false))->union (

(self.activities.createprocessinstance- $>\operatorname{select}(a \mid a=$ true $)$ ))->size ()$=0$ 
As Figure 6.11 shows, since the Activity Container primitive is "part of" the Process Context primitive, a composition relationship between these two classes. With regard to the definition of an Activity Container, this defines four attributes to indicate (1) an internal identifier (id attribute), (2) the name used to be displayed to the user (alias attribute), (3) if the Activity Container corresponds to the first activity of the process requiring interaction with humans (initial attribute) and (4) if the Activity Container corresponds to the first activity of the process (createProcessInstance attribute).

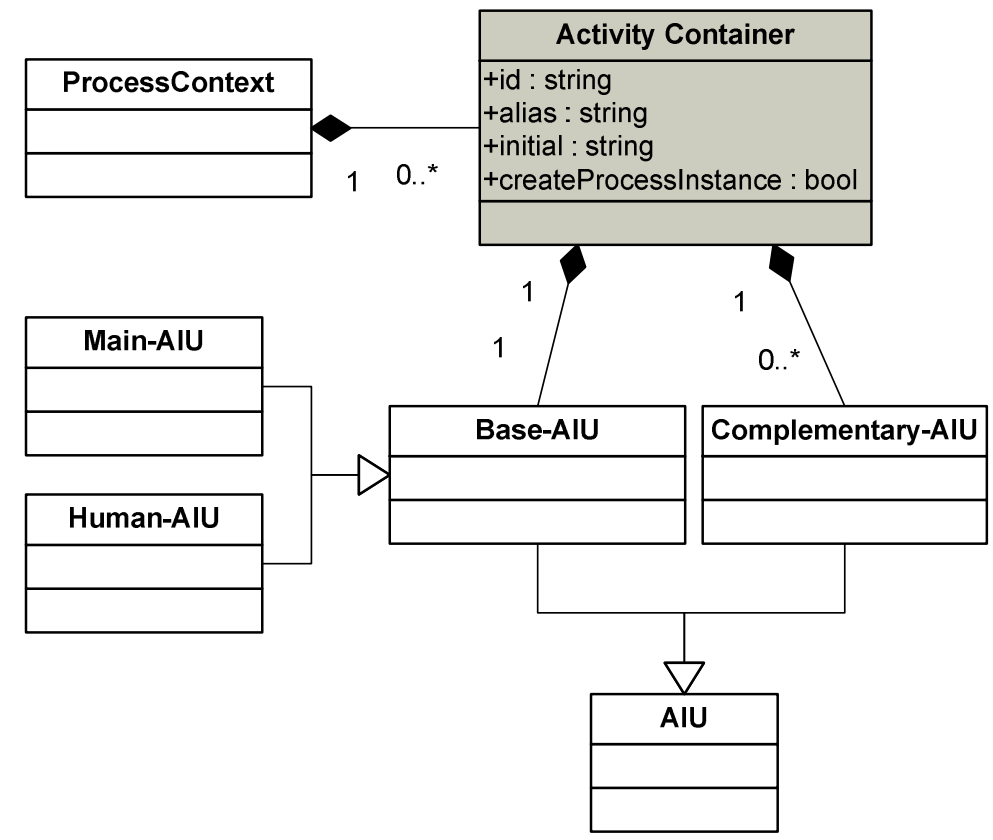

Figure 6.11 Metamodel Excerpt including the Activity Container primitive 


\subsubsection{Main-AIU}

Problem: We need to specify which data has to be necessarily provided to the user to complete a "user" BP activity (an activity performed by the user that changes the state of the system).

Concrete Syntax: In Figure 6.2 and Figure 6.4 we can see different Main-AIUs which are associated to their corresponding Activity Containers. For instance, the "Validate Request" Activity Container includes a "ValidateRequest" Main-AIU. This primitive gathers the data and functionality that has necessarily to be provided to the user to complete the associated BP activity. In the case of the "ValidateRequest" Main-AIU, the data and functionality is gathered from the class-views ("RequestBook" and "Member") defined within the Main-AIU.

A generic diagram of this primitive is shown in Figure 6.12. As this figure shows, a Main- $A I U$ is depicted graphically as a white square divided by a single line (see the white element of Figure 6.12). The top section is dedicated to gather views over the Structural and Services model. The bottom section is dedicated to define an Index (see subsection 6.3.6). It includes the keyword «Main-AIU» followed by the name of the unit. 


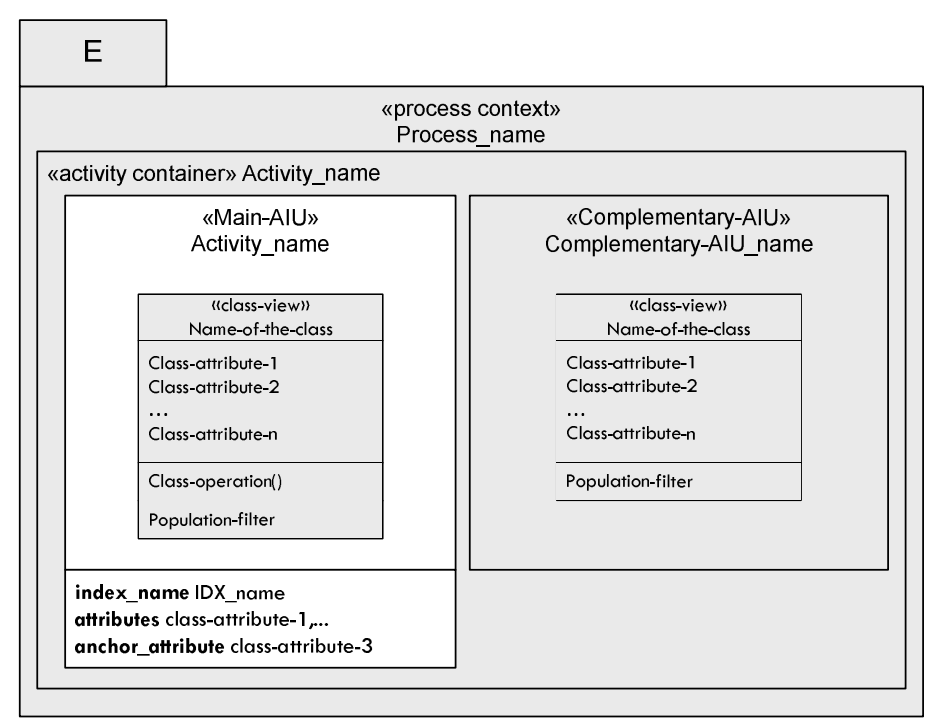

Figure 6.12 Main-AIU primitive

Abstract Syntax: This primitive gathers all the data and functionality required by the user to perform a specific task. It is divided into two sections; (1) one that includes views over the Structural model (see Class-view) or the Services model (see Service-functinoal-view) and (2) another that defines the way data is going to be shown when the AIU retrieves more than one instance (see the Index primitive presented in section 6.3.6).

The Main-AIU primitive has been defined as a specialized class of the Base-AIU abstract class. The reason to introduce this abstract class is to represent the constraint previously presented that states that "An Activity Container can only include either one Main-AIU or one Human-AIU”. In turn, this abstract class inherits from the AIU class which defines attributes to represent (1) an internal identifier (id attribute), (2) a string used for 
identifying the AIU at the user interface (alias attribute) and (3) the relationship between this AIU and the Context in which this AIU belongs (isContextual attribute).

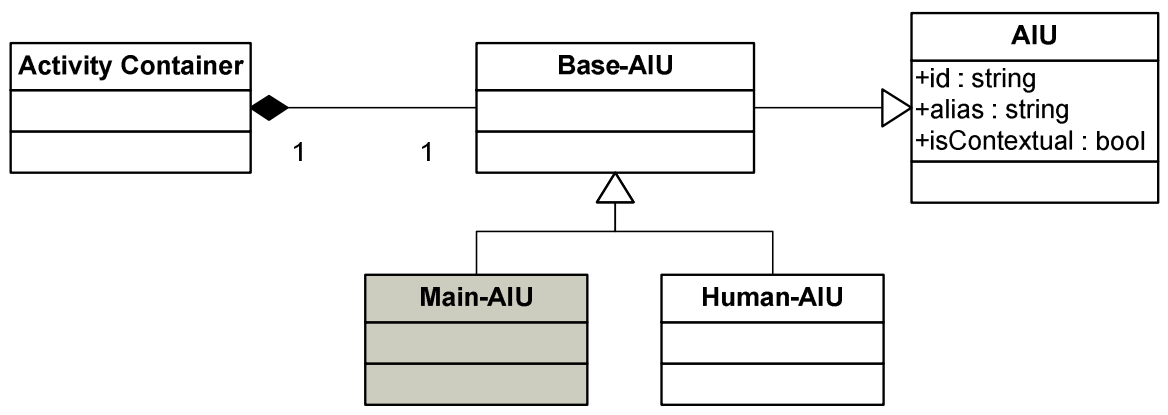

Figure 6.13 Metamodel Excerpt including the Main-AIU primitive

A particularity introduced by this primitive is that within a Main$A I U$, the included views (class-views or service-functional-views) can only specify one operation. Specifically, this operation corresponds to the functionality that supports the associated $\mathrm{BP}$ activity. This constraint is expressed in the OCL language as follows:

\section{Context MainAIU}

inv: (self.ManagerClass.NavigationalOperation->size ()$=1$ ) or (self.ComplementaryClass.NavigationalOperation->size ()$=1$ )

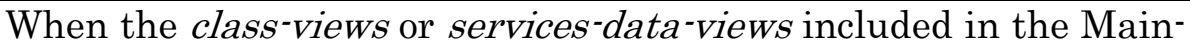
AIU retrieve more than one instance, the Main-AIU primitive activates the Index defined in its bottom section. As a result, it filters the data defined in the views and shows just the attributes specified in the attribute section of the Index. 


\subsubsection{Human-AIU}

Problem: We need to specify which data has to be necessarily provided to the user to complete a "human" BP activity.

Concrete Syntax: In Figure 6.2 we can see the "PickUpBooks" Human-AIU associated to the "PickUpBooks" Activity Container. This primitive gathers the data that has necessarily to be provided to the user to complete the associated BP activity. In this case, the data is gathered from the class-views defined within the HumanAIU.

Figure 6.14 shows how the Human-AIU modelled in Figure 6.2 is implemented in a Web page. In this case, the event associated to the "submit" button does not invoke any functionality. It only communicates the BP that the current BP activity has been completed. 


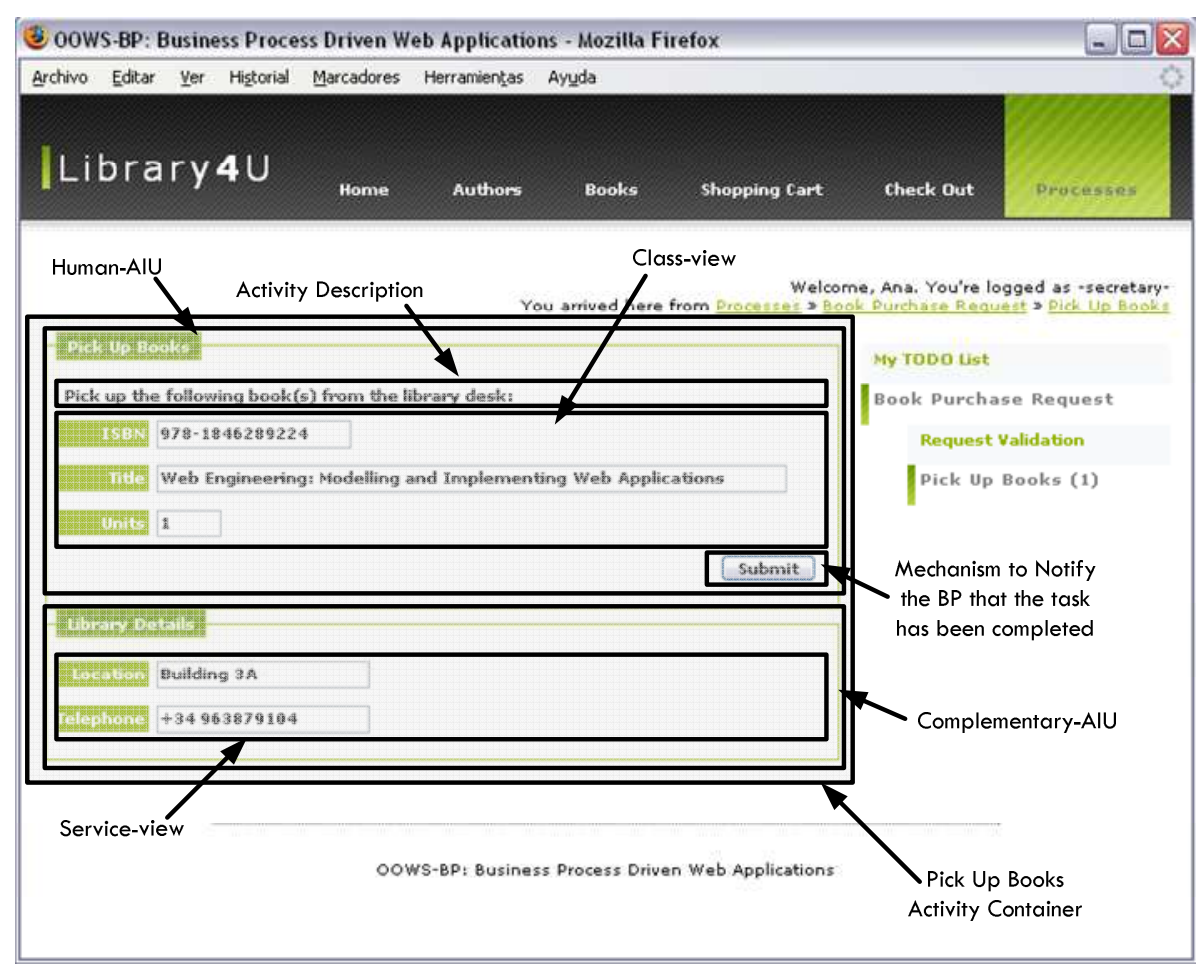

Figure 6.14 Implementation of a Human-AIU

A generic diagram of this primitive is shown in Figure 6.15. As this figure shows, a Human-AIU is depicted graphically as a white rectangle that includes the keyword «Human-AIU» followed by the name of the unit (see the white part of Figure 6.15). Moreover, this primitive display data related to the activity being performed (usually a description of the tasks to perform). 


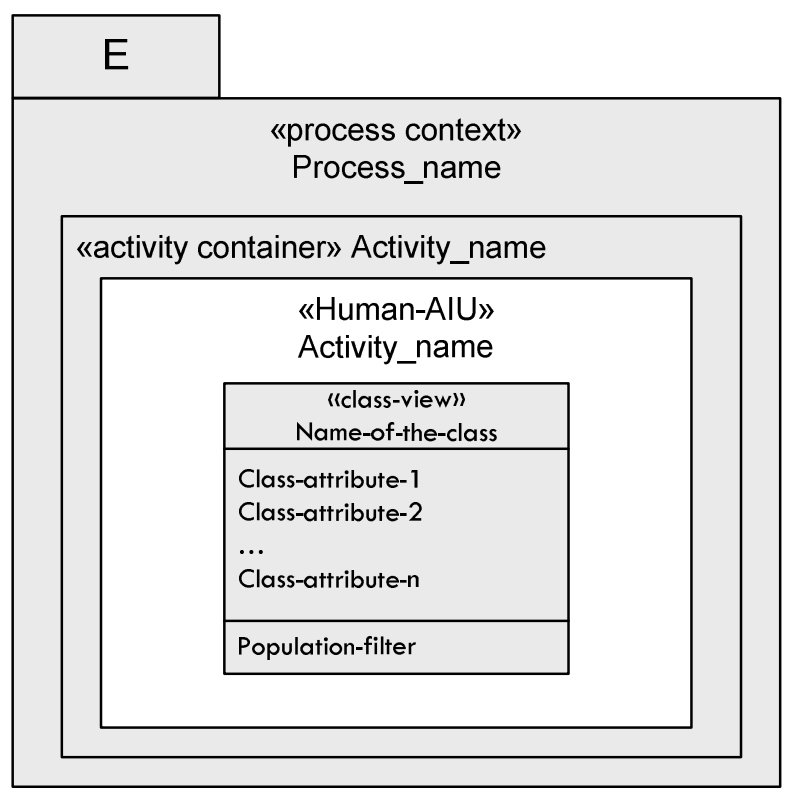

Figure 6.15 Human-AIU primitive

Abstract Syntax: This primitive represents BP activities defined in the BPM that are not automated in the system (activities that are fully performed without the assistance of any system).

Similar to the Main-AIU primitive, the Human-AIU primitive has been designed as a child class from the Base-AIU abstract class. In addition to the attributes inherited from the AIU class, the Human-AIU primitive includes the description attribute which is used to include information that is going to be presented to the user when the corresponding Human-AIU gets activated. 


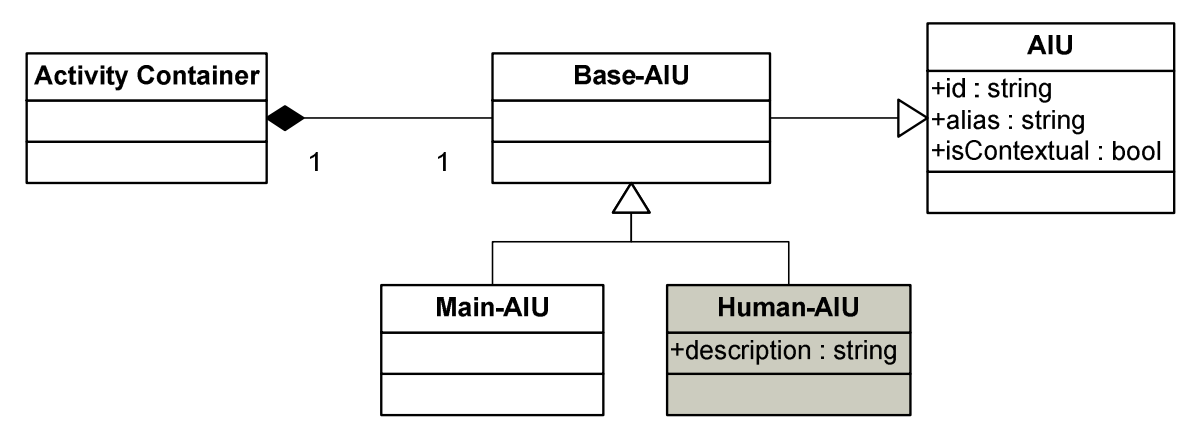

Figure 6.16 Metamodel Excerpt including the Human-AIU primitive

This primitive represents a BP activity that is not automated in the system. Therefore, the views defined in it cannot include any operation. This constraint is expressed in the OCL language as follows:

Context HumanAIU

inv: (self.ManagerClass.NavigationalOperation->isEmpty() and self.ComplementaryClass.NavigationalOperation->isEmpty())

\subsubsection{Complementary-AIU}

Problem: We need to specify the information that can "help" the user in completing a specific BP activity. Providing the user with this information we avoid the user suspending the current activity to look for specific information through content navigation. As a result, we are reducing the amount of time spent by the user to finalize the BP activity.

Concrete Syntax: In Figure 6.2 we can see that both Activity Containers include Complementary-AIUS. This primitive gathers information that assists the user to complete the associated BP 
activity. In this case, the information is retrieved by means of class-views and service-data-views defined within the corresponding Activity Containers.

A generic diagram of this primitive is shown in Figure 6.17. As this figure shows, a Complementary-AIU is depicted graphically as a white rectangle that includes the keyword «ComplementaryAIU» followed by the name of the unit (see the white part of Figure 6.17).

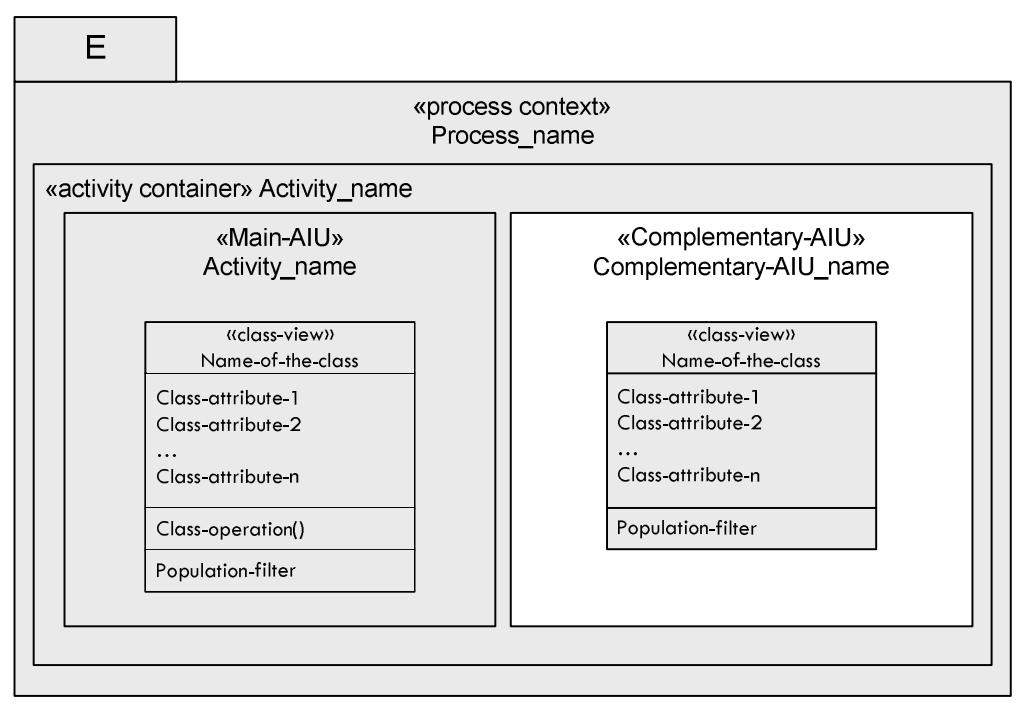

Figure 6.17 Complementary-AIU primitive

Similar to Human-AIUS, this primitive gathers views over the system (from the Structural and Services models) with the particularity that no functionality can be defined in it.

Abstract Syntax: This primitive was introduced to assist the user while she is performing a specific task. It provides additional information that may help her in completing it. This AIU is not 
strictly necessary to perform the task but avoids the user leaving the execution of the process to reach this information through content navigation. The objective of this AIU is to speed up the completion of the process providing the user with useful information. Contrary to other primitives, this one cannot be derived automatically from the $\mathrm{BP}$ definition. In this case, the developer has to include it manually. For this reason, during the specification of the BP, the analyst specifies (in a textual form) the information that should be provided associated to each BP activity. This textual description will be used by the developer to decide the complementary units that should be included for each Activity Container.

Complementary-AIUs are optional units within a particular Activity Container. This constraint is defined by the multiplicity of the composition association defined between these two primitives. Moreover, the Complementary-AIU class inherits the three attributes defined in the AIU class.

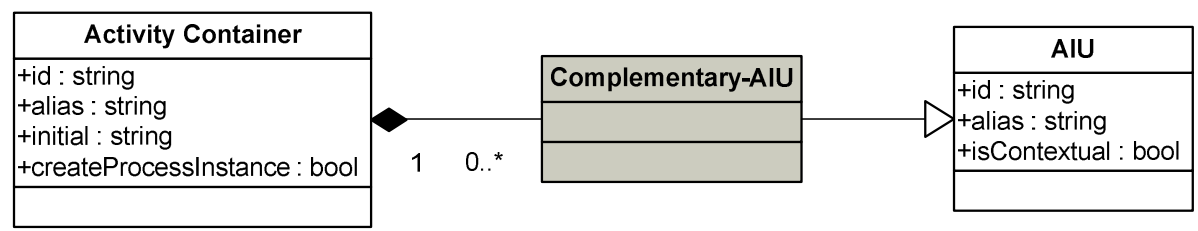

Figure 6.18 Metamodel Excerpt including the Complementary-AIU primitive

Since Complementary-AIUS are used to provide some useful data related to the current $\mathrm{BP}$ activity, the views defined in it cannot specify any operation. These views can only retrieve information, 
no functionality. This constraint is expressed in the OCL language as follows:

Context ComplementaryAIU

inv: (self.ManagerClass.NavigationalOperation->isEmpty() and

self.ComplementaryClass.NavigationalOperation->isEmpty())

Related to the previous constraint, since the Service-FunctionalView is a primitive used to provide functionality to the user, this primitive cannot be associated to a Complementary AIU. This constraint is expressed in the OCL language as follows:

Context ComplementaryAIU

inv:

(self.NavigationalClass.ManagerClass.ServiceFunctionalView->isEmpty()) and

(self.NavigationalClass.ComplementaryClass.ServiceFunctionalView>isEmpty())

\subsubsection{Index}

Problem: During the execution of a long-running process, we need to specify the information that is going to be included in the GUI in two different contexts (1) when the GUI gathers all the instances of a particular BP activity and (2) when the GUI gathers just one BP activity instance. This is necessary since different BP instances can exist for the same long-running BP.

Concrete Syntax: Figure 6.2 illustrates how an Index is associated to the "Validate Request" Main-AIU. In this case, the 
“ATTRIBUTES" section specifies that the book title, units and the login of the applicant member are going to be retrieved for each instance retrieved by the Main-AIU. In addition, the "LINK ATTRIBUTE" section states that the "title" field is going to be used as anchor to reach the selected instance.

A generic diagram of this primitive is shown in Figure 6.19. As this figure shows, an Index is depicted graphically as a white box attached to the bottom section of a Main-AIU (see the white part of Figure 6.19). Its definition is merely textual and includes three attributes which are (1) the index name, (2) a list of attributes that are shown when more than one instance is retrieved and (3) an attribute that is used as anchor to retrieve the discriminated data of a particular instance. Only the attributes included in the attributes list will be displayed when the associated AIU retrieves more than one instance.

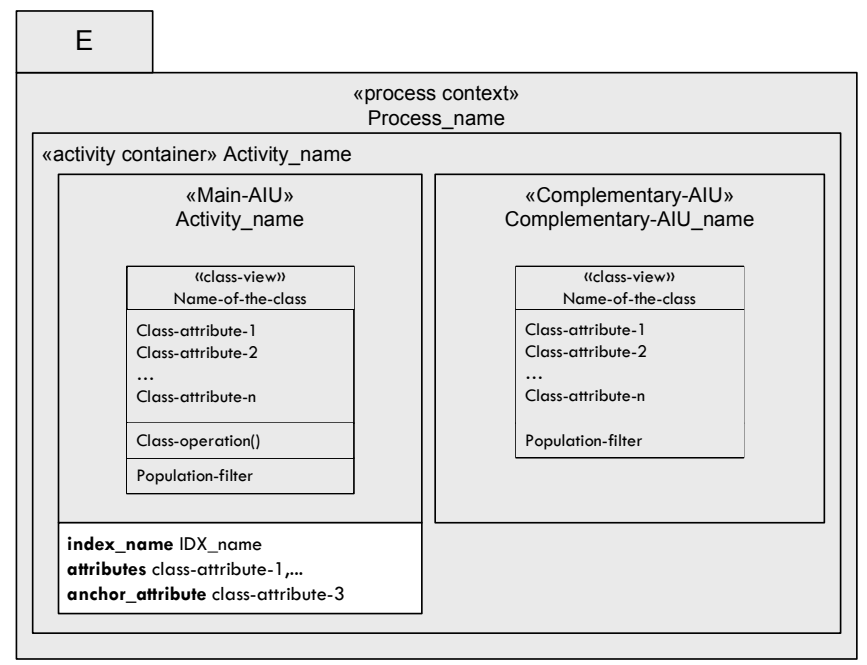

Figure 6.19 Index primitive 
The Index primitive is always associated to the Main-AIU and Human-AIU primitives. This is because these primitives can potentially gather more than one activity instance in the same GUI. This constraint is expressed in the OCL language as follows:

\section{Context MainAIU}

inv: self.ManagerClass.Index->size ()$=1$

\section{Context HumanAIU}

inv: self.ManagerClass.Index->size ()$=1$

Figure 6.20 and Figure 6.21 show the difference between an activated and deactivated index. On the one hand, Figure 6.20 shows the "Request Validation" Main-AIU with the index activated. As a result, only the title, units and solicitor data is shown for the active instances of this $\mathrm{BP}$ activity. On the contrary, Figure 6.21 shows the same AIU but this time with the index deactivated. As a result, one activity instance is shown with all the information specified in the corresponding view (title, editor, publisher, year, solicitor and units). 


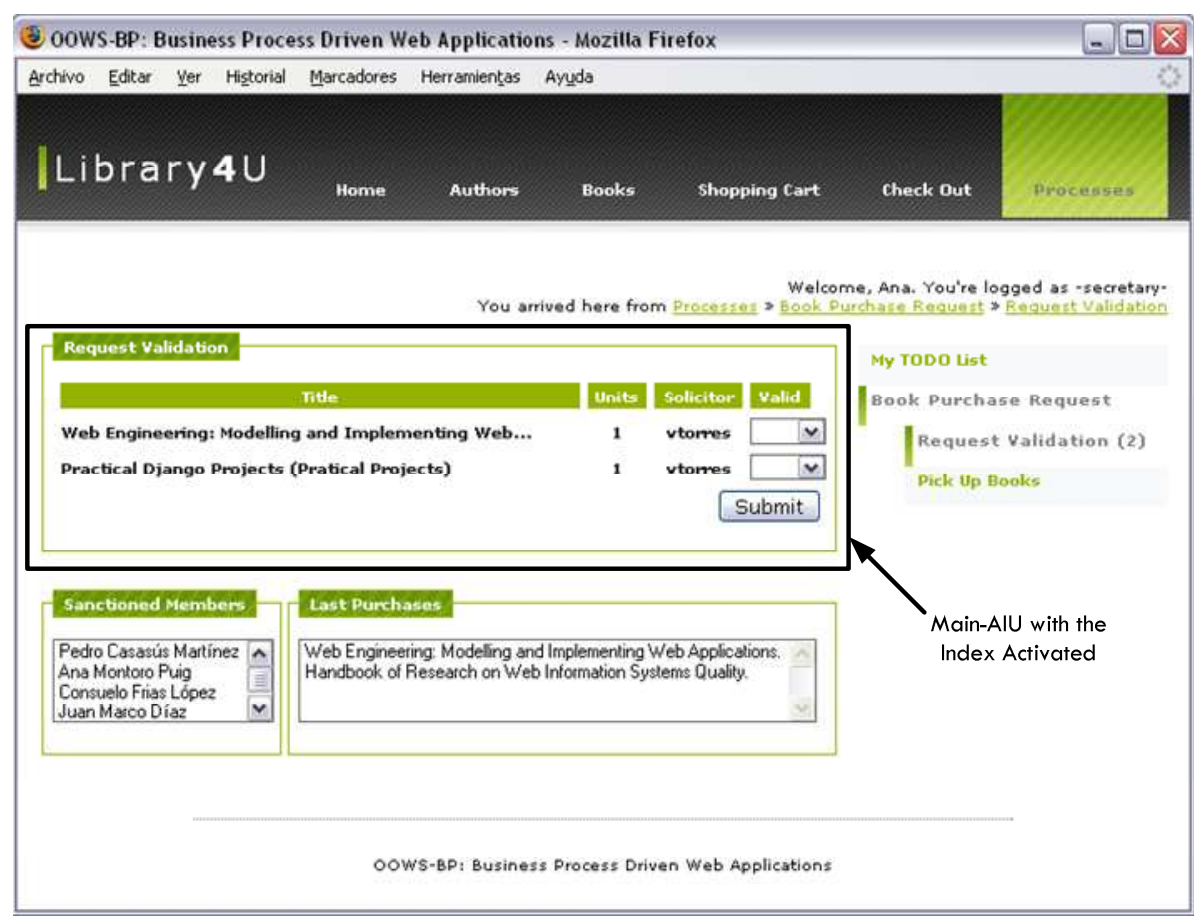

Figure 6.20 Implementation of a Main-AIU when the index gets activated

The main difference between these two contexts is that it allows handling multiple process instances (when the index gets activated) or just one process instance (when the index is deactivated). 


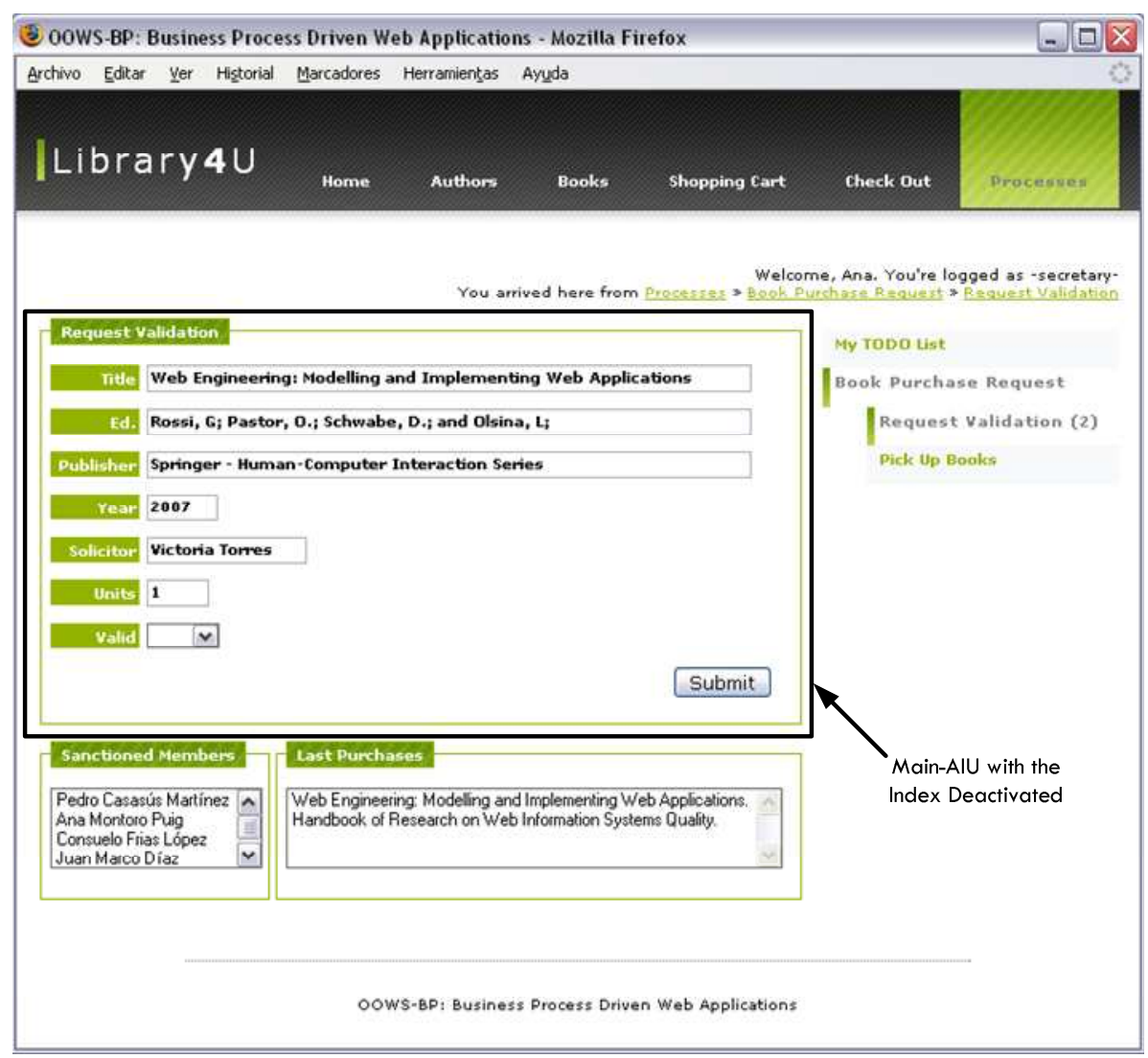

Figure 6.21 Implementation of a Main-AIU when the index gets deactivated

Abstract Syntax: An Index primitive is used to reduce the amount of data that is retrieved by a Main-AIU when this retrieves more than one instance. The reduction is carried out by limiting the amount of data displayed by each instance. An Index gets activated automatically when the corresponding Main-AIU retrieves more than one instance. Then, the attribute defined as anchor can be used to access all the associated and not displayed data of a particular instance. On the contrary, when the Main-AIU simply retrieves one instance, the index does not get activated and 
the whole content (no instance data is discarded) is displayed to the user.

The set of attributes defined by the Index attribute (attributes and anchor) are referred to the NavigationalAttributes defined within the associated AIU (see Figure 6.22). Indexes are always activated automatically and this activation depends on the state of the AIU. When the AIU retrieves a population the index gets activated automatically. On the contrary, when the AIU retrieves the info for a particular instance, the index gets deactivated.

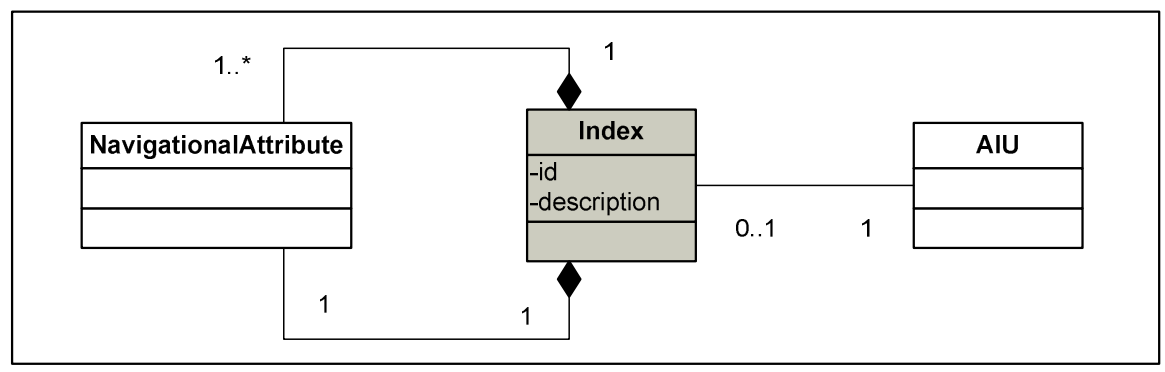

Figure 6.22 Metamodel Excerpt including the Process-Index primitive

\subsubsection{Class`view}

Problem: We need to specify which data and functionality provided by the "local system" is going to support a specific BP activity.

Concrete Syntax: Figure 6.2 and Figure 6.4 show different classviews defined within the corresponding AIU. For instance the "Validate Request" Main-AIU illustrated in Figure 6.2 includes the "RequestBook" class-view. According to the constraint associated to the Main-AIU which states that the views included in it can only specify one operation, this view only specifies the "validateRequest()" operation. Moreover, it also defines a 
population filter which specifies that only the instances whose "state" attribute is valued either to the "pending" or "denied" values are retrieved by the class-view.

A generic diagram of this primitive is shown in Figure 6.23. As this figure shows, a $\mathrm{Class}^{-}$view is depicted graphically as a white square divided into four sections which define (1) the name of the class being retrieved; (2) the class attributes that are going to be retrieved; (3) the operation that supports the BP activity; and finally (4) the filter over the population retrieved by the class view (see the white part of Figure 6.23).

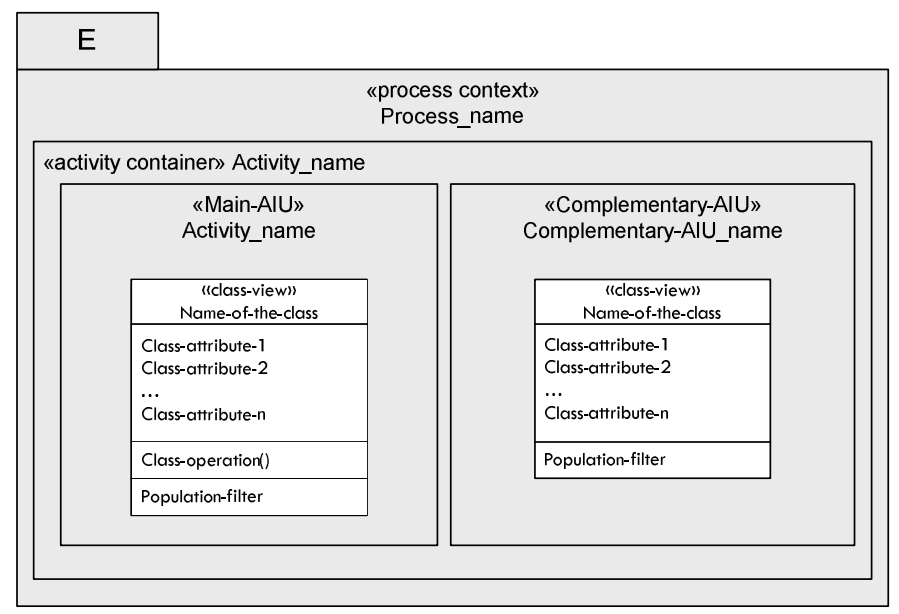

Figure 6.23 Class-View primitive

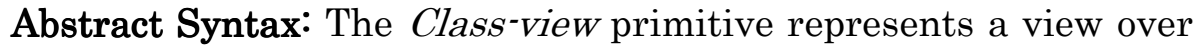
a class from the Structural Model. This view is made up of four parts; (1) the top section which includes the name of the class being retrieved; (2) the second section that includes the class attributes that are going to be retrieved; (3) the third section which includes the operation that supports the task; and finally 
(4) the bottom section that defines a filter over the population retrieved by the class view. In general, Class-views can include several operations in their third section. However, when these are used for BPs definition they can only include one operation corresponding to the associated process task (when this view is contained within a Main-AIU) or zero operations (when it is contained within a Complementary-AIU).

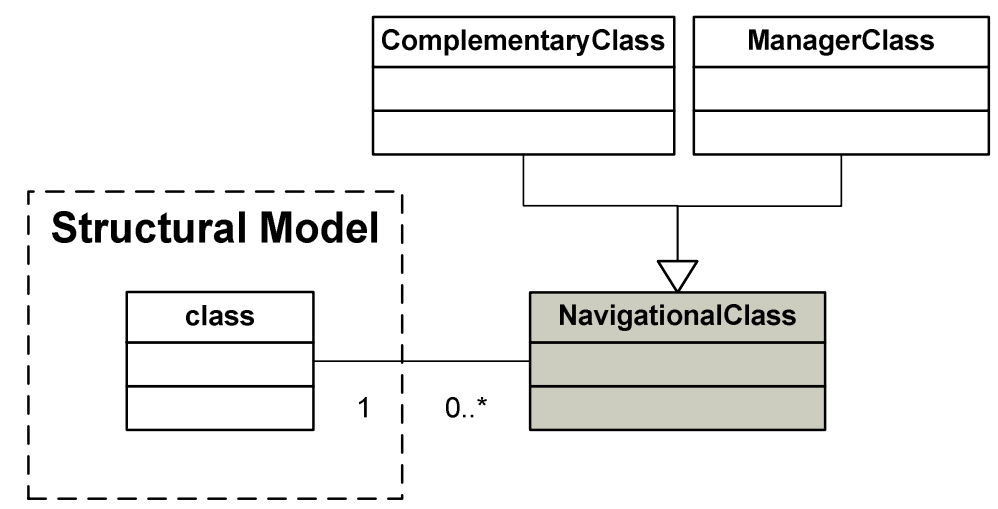

Figure 6.24 Metamodel Excerpt including the NavigationalClass primitive

Depending on the context, a Class-view can retrieve either a particular class instance (when the class-view is passed with an object reference) or the class population (when no object reference is passed). Moreover, the retrieved instances have always to satisfy the filter condition in case this has been defined.

\subsubsection{Service-Data-view}

Problem: We need to specify which data provided by "external systems" is going to support a specific BP activity. 
Concrete Syntax: Figure 6.2 shows the Service-Data-View defined over the "CentralLibraryWS". In this case, the Service-Data-View retrieves the "location" and the "telephone" data from the data returned by the "getOrganizationDetails0" operation.

A generic diagram of this primitive is shown in Figure 6.25. As this figure shows, a Service-Data-View is depicted graphically as a white square divided into three sections which specify (1) the name of the service being invoked; (2) the fields that are returned by the operation and that are going to be displayed to the user and (3) the service operation being invoked (see the white part of Figure 6.25).

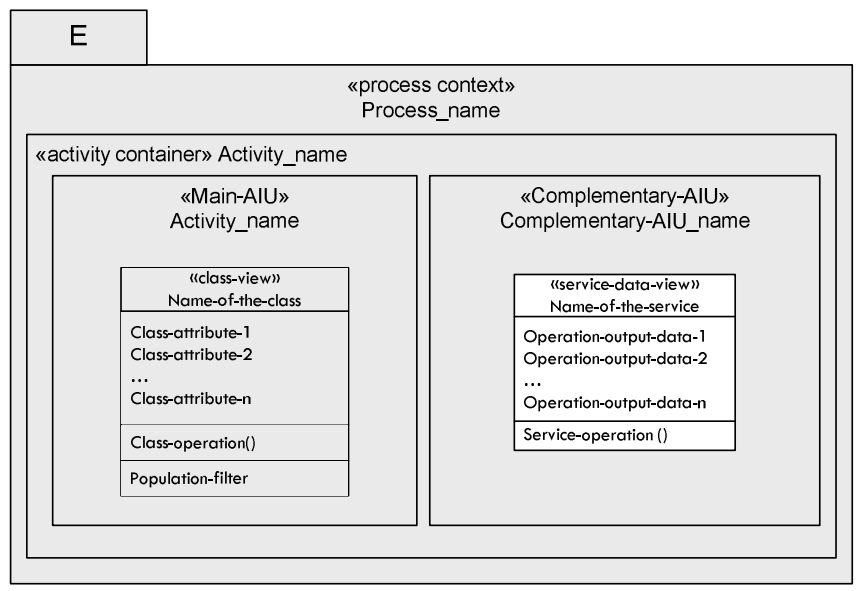

Figure 6.25 Service-View primitive

Abstract Syntax: The Service-Data-view primitive represents a view over a service operation from the ones imported into the Services Model. 
As Figure 6.26 shows, the Service-Data-view primitive has been defined as a specialized class of the abstract ServiceView class (class representing views over the services imported into the Services Model).

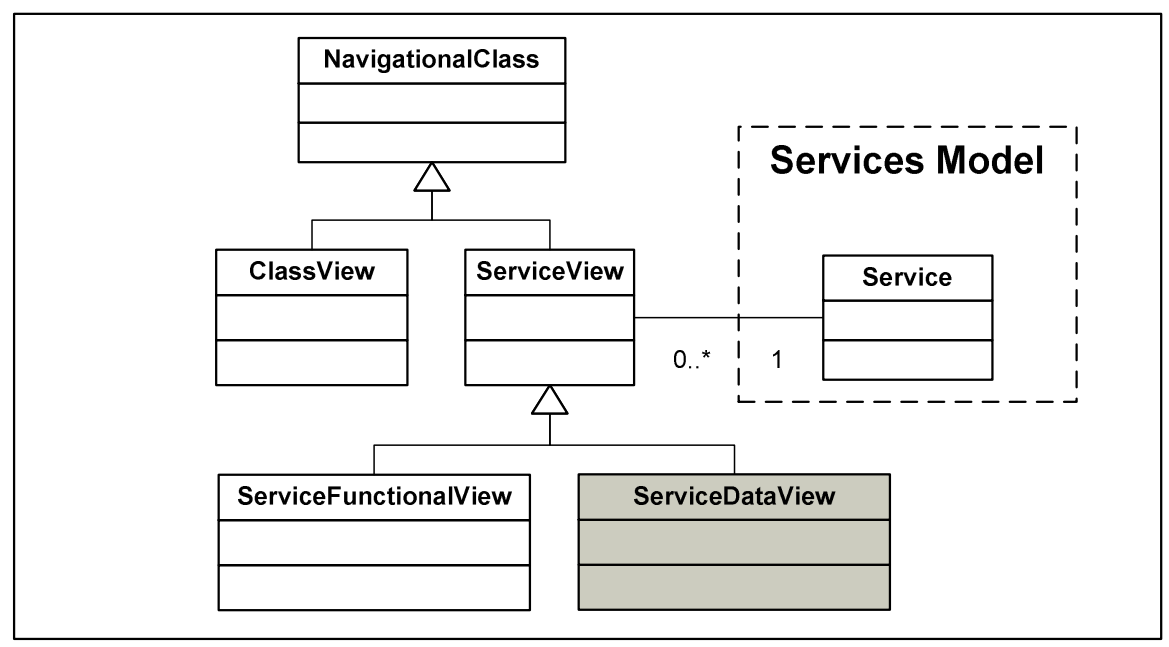

Figure 6.26 Metamodel Excerpt including the Service-data-view primitive

The Service-view primitive invokes a service operation and gathers the returned values to be displayed to the user.

\subsubsection{Service-Functional-View}

Problem: We need to specify which functionality provided by "external systems" is going to support a specific BP activity.

Concrete Syntax: Figure 6.4 and Figure 6.27 show respectively how service-functional-views are used in the navigational model and how these are finally represented at the implementation level. In particular, in Figure 6.4 the "Payment" Activity Container 
includes within its Main-AIU a Service-Functional-view linked to the payment operation kept in the Services Model.

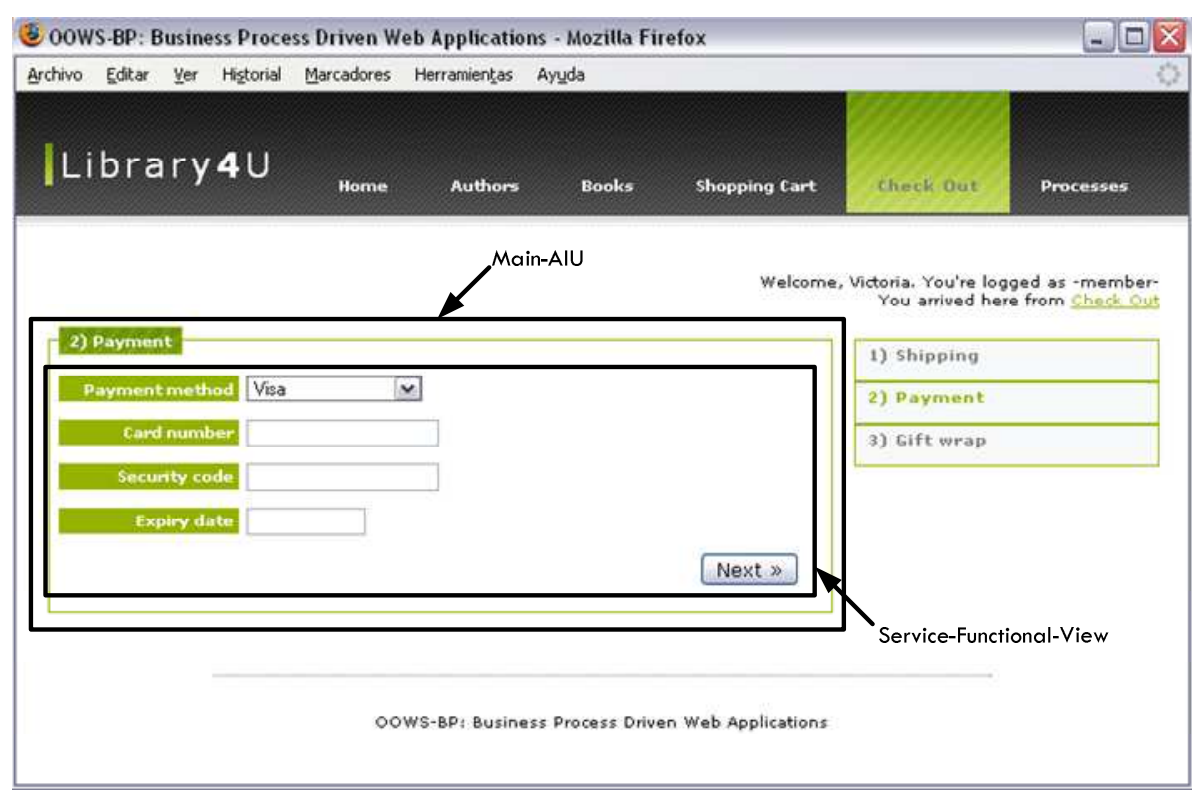

Figure 6.27 Web page corresponding to the "Payment" Activity Container

A generic diagram of this primitive is shown in Figure 6.28. As this figure shows, a Service-Functional-View is depicted graphically as a white square divided into two sections which specify (1) the name of the service being invoked and (2) the operation being invoked (see the white part of Figure 6.28). 


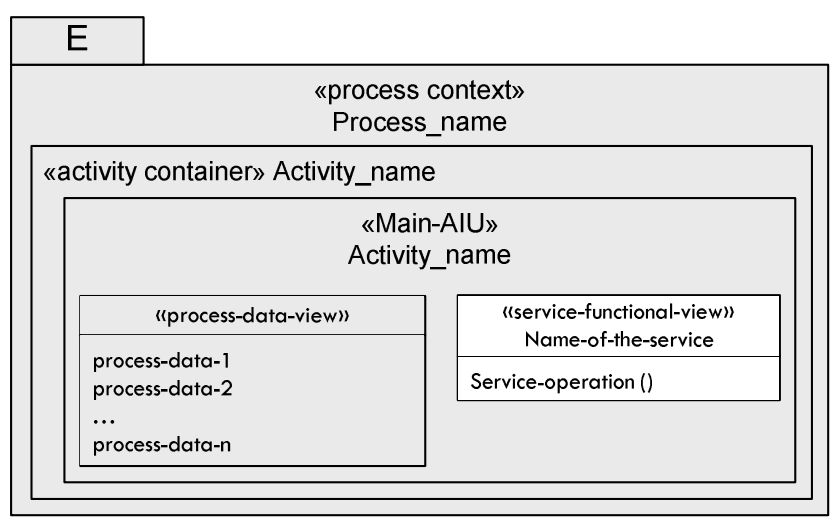

Figure 6.28 Service-Functional-View primitive

\begin{abstract}
Syntax: Similarly to the Service-Data-view primitive, the Service-Functional-view primitive represents a view over a service operation from the ones imported into the Services Model. The difference between this primitive and the Service-Data-view primitive is that this is used when the service is invoked and no returned data has to be displayed to the user. This does not mean that the service is not going to return any data. However, none of the returned data is going to be shown to the user. This view is made up of two parts; (1) the top section which includes the name of the service being invoked; (2) the bottom section that defines the operation being invoked.
\end{abstract}




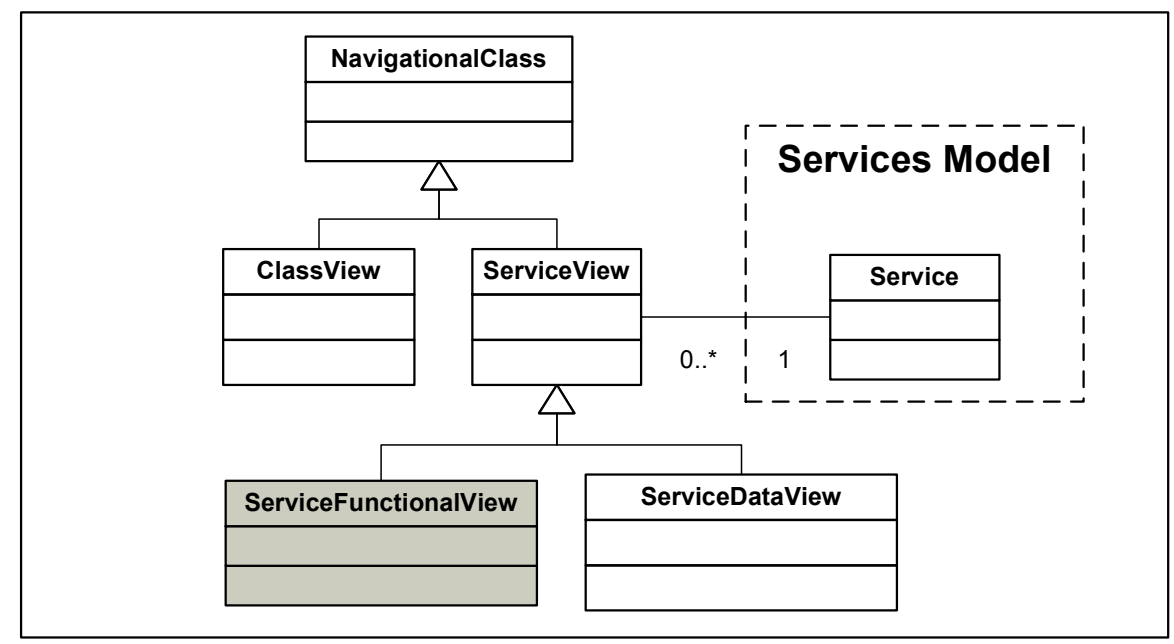

Figure 6.29 Metamodel Excerpt including the Service-functional-view

\subsubsection{Process-link}

Problem: We need to specify the entry points that allow launching a specific $\mathrm{BP}$.

Concrete Syntax: Figure 6.3 showed that the "Checkout" Process Context was defined as "Sequence" context. This means that this Process Context is reached after traversing the "Shopping Cart" Navigational context. However, to reach the "Checkout" Process Context is necessary to include a mechanism that allows linking these two contexts. As Figure 6.30 shows, this mechanism is the Process-link primitive. Associated to this primitive we have to specify (1) the anchor to invoke the process (the "checkout" string over the solid arrow) and (2) the process that is going to be invoked (the "CheckOut" process). As a result, an entry point to the initial Activity Container defined in the process is defined. 


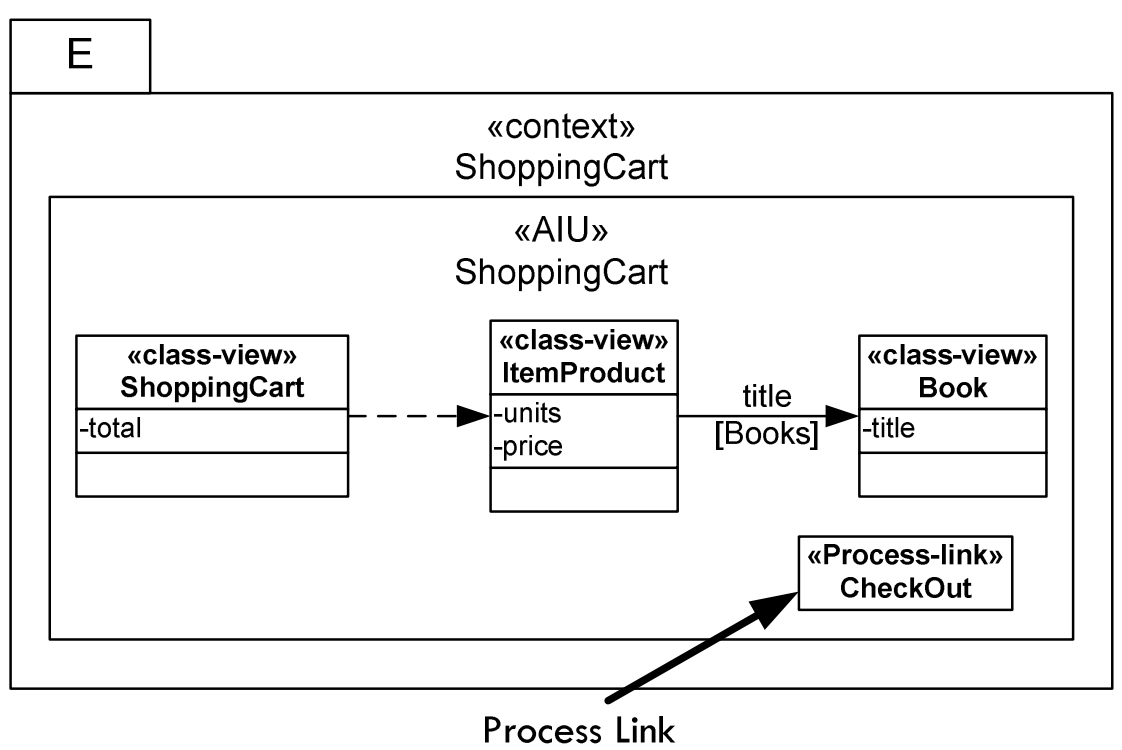

Figure 6.30 Detail of a Navigational Context including a process-link

It is necessary to specify the activity to be invoked because the navigational model does not define relationships between $\mathrm{BP}$ activities (this is only kept by the $\mathrm{BP}$ handled by the process engine). Figure 6.31 illustrates the Web page corresponding to the "Shopping Cart" Navigational Context. In this Web page, the entry point to the "Check Out" BP is provided by means of the "Check Out" link included at the right hand of the Web page. 


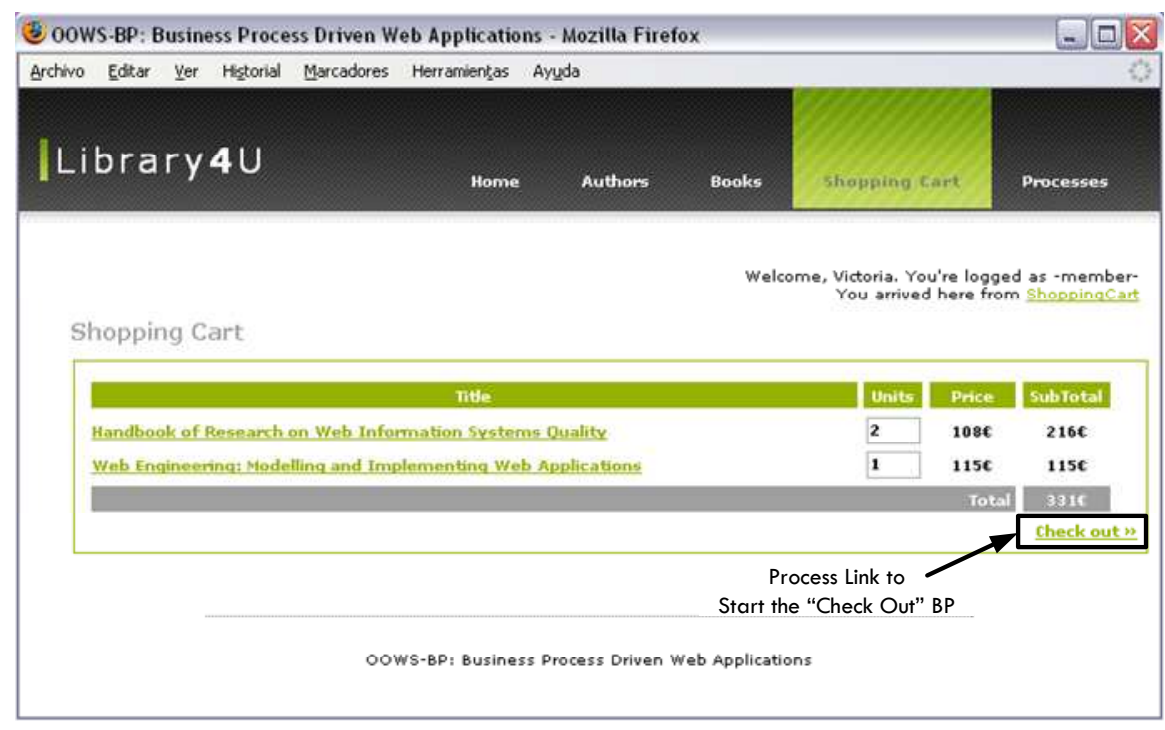

Figure 6.31 Web page corresponding to the Shopping Cart Navigational context

A generic diagram of this primitive is shown in Figure 6.32. As this figure shows, a Process-Link is depicted graphically as a rectangle (see white part of Figure 6.32) which includes the keyword «process-link» and the name of the process being invoked. Moreover, the primitive includes a property that allows indicating the text used as anchor to activate the process. 


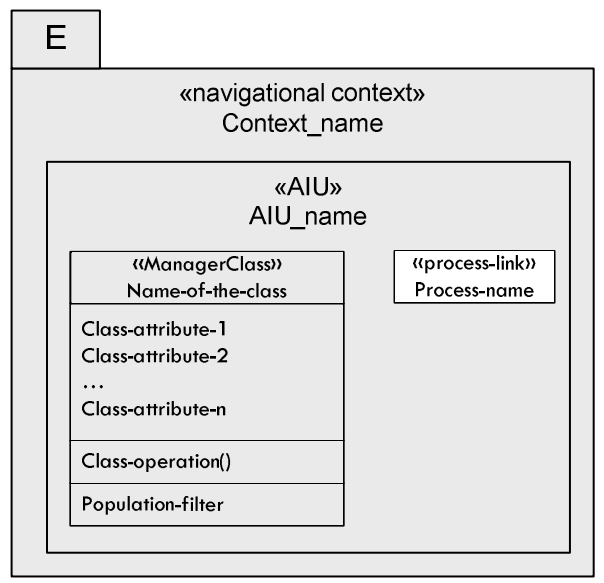

Figure 6.32 Process-Link primitive

Abstract Syntax: The Process-link primitive is used to invoke a process context that has been defined as Sequence context. Therefore, it defines the entry point to a specific business process. This primitive is linked with the AIU construct which will include it. Since one Activity Container from the referenced process is going to be defined as initial, it is not necessary to specify the Activity Container that has to be activated.

The ProcessLink primitive is associated with one ProcessContext. Moreover, ProcessLinks can only exist within an AIU.

\begin{tabular}{|c|c|c|c|c|}
\hline ProcessContext & & ProcessLink & & AIU \\
\hline & & +alias : string & & \\
\hline & $1 \quad 0 .{ }^{*}$ & & 0..* ${ }^{*} .^{*}$ & \\
\hline
\end{tabular}

Figure 6.33 Metamodel Excerpt including the Process link primitive 


\subsection{Extended Navigational Metamodel}

This section presents the primitives presented in the previous section but this time integrated with the original ones (just the excerpt related to the former primitives is included). This metamodel is defined as the UML class diagram presented in Figure 6.34 together with the set of OCL constraints that have been presented along section 6.3.

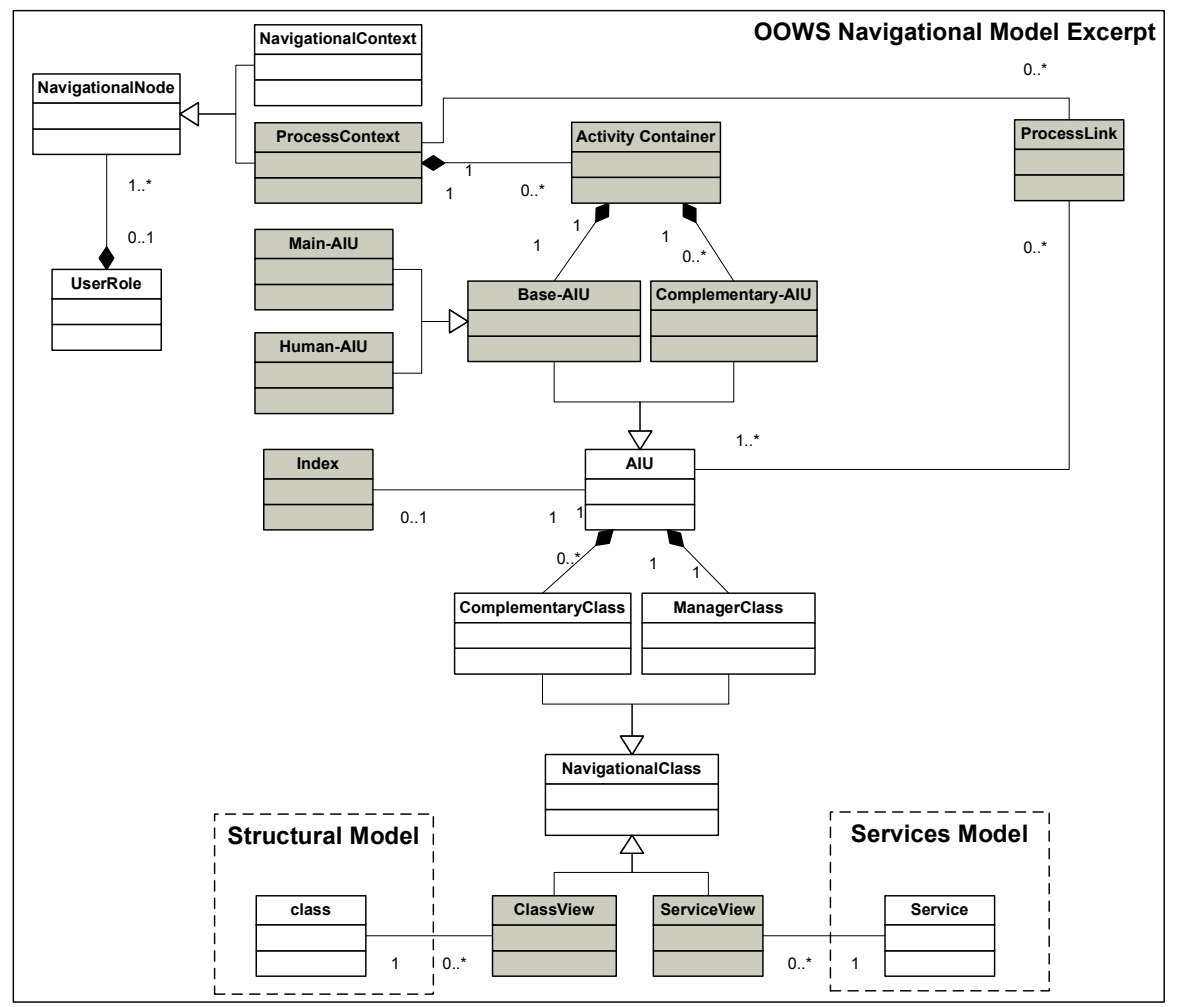

Figure 6.34 Navigational Model Excerpt including the BP related primitives

All the gray-coloured primitives depicted in Figure 6.34 correspond to the primitives related to the BP extension. 


\subsection{Conclusions}

This chapter has presented the extension defined for the OOWS Navigational model to deal with BP integration. The presented new primitives extend the conceptual modeling stage allowing a correct description of web systems dealing with the execution of BPs.

As we already stated in chapter 2, the objectives of the navigational model are the following three ones (1) defining the structure of the web application, (2) defining the way this structure can be accessed and (3) defining the content and functionality that should be provided by the web application. However, the extension presented in this chapter only deals with the first and third objectives of this model. The main reason to leave out the second one is that the navigation during $\mathrm{BP}$ execution is going to be driven by the process.

Moreover, apart from enriching the expressivity of the model with new primitives, the extension enforces the relationship with other models. In particular, new relationships with the Services Model have been defined. This allows retrieving data and functionality not only from our system but also from external ones. 


\section{Chapter 7}

\section{Presentation Model Extension}

The previous chapter was dedicated to present the Navigational extensions that are necessary to deal with the specification of BPs in Web applications. In the current chapter we present the extensions that are required in the Presentation model to better adequate the Navigational contents during BP execution. Since the Presentation model is based on a set of patterns, the extension proposed involves the definition of new patterns that allow improving the user experience while executing BPs. In addition, to face the drawbacks found in existing Web engineering methods to deal with usability issues (Atterer et al., 2006), this chapter presents how these issues can been integrated into the OOWS Web Engineering method to ensure the quality of the generated web systems.

\subsection{Introducing the New Presentation Primitives}

Before detailing the new primitives, this section introduces them graphically. This is performed by using the Book Purchase Request BP. Figure 7.1 shows the presentation primitives associated to the process context of the example. 


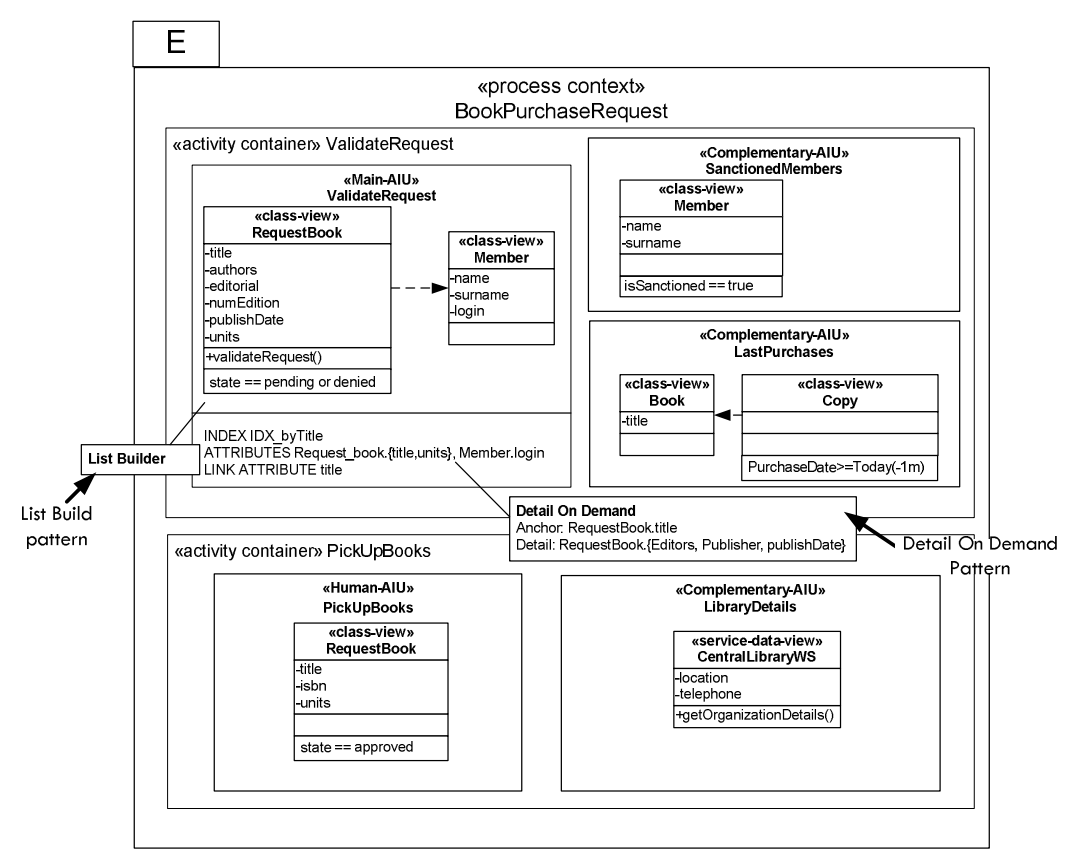

Figure 7.1 Presentation attributes associated to the Book Purchase Request BP

As Figure 7.1 shows, the navigational elements included in the process context have associated some presentation attributes. These will be used during the transformation process to generate the layout of this context accordingly.

\subsection{Presentation Primitives to Support BP execution}

The extension defined over the Presentation metamodel defines two new primitives that are associated to the GUI generated for the execution of BPs. These new primitives correspond to the Details On Demand and List Builder patterns. Each primitive is going to be presented according to this schema: First, the problem that motivates the definition of each primitive is presented. 
Second, the concrete syntax of the primitive is presented. This syntax is described in a diagrammatic form and defines how the abstract syntax (which is presented next) is presented to the end user. Finally, the abstract syntax of the primitive is presented. In this case, the concepts associated to the primitive and their relationships to other primitives (even from another metamodel) are described.

\subsubsection{Details On Demand Pattern}

Problem: The amount of data that can potentially be of interest to users enforces developers to organize this data according to the GUI limitations. Therefore, users have to follow different links to reach the data that was discarded to overload the GUI. More lightweight mechanisms are necessary to reach this discarded/ hidden data.

Concrete Syntax: According to Figure 7.1, the index associated to the "Validate Request" Main-AIU includes a "Details On Demand" pattern. In this case, the title attribute included in the "RequestBook" class-view has been defined as anchor to retrieve the information specified in the detail section of the pattern (the editors and publisher attributes from the RequestBook class-view).

Figure 7.2 shows how this pattern is presented in a web page (web page according to the process context depicted in Figure 7.1). In this case, each title item defines a link with the following behaviour: when the user positions the mouse over the title, new contents related to the item are shown in a new floating section. In 
this particular case, Author, Publisher and Publish date are shown.

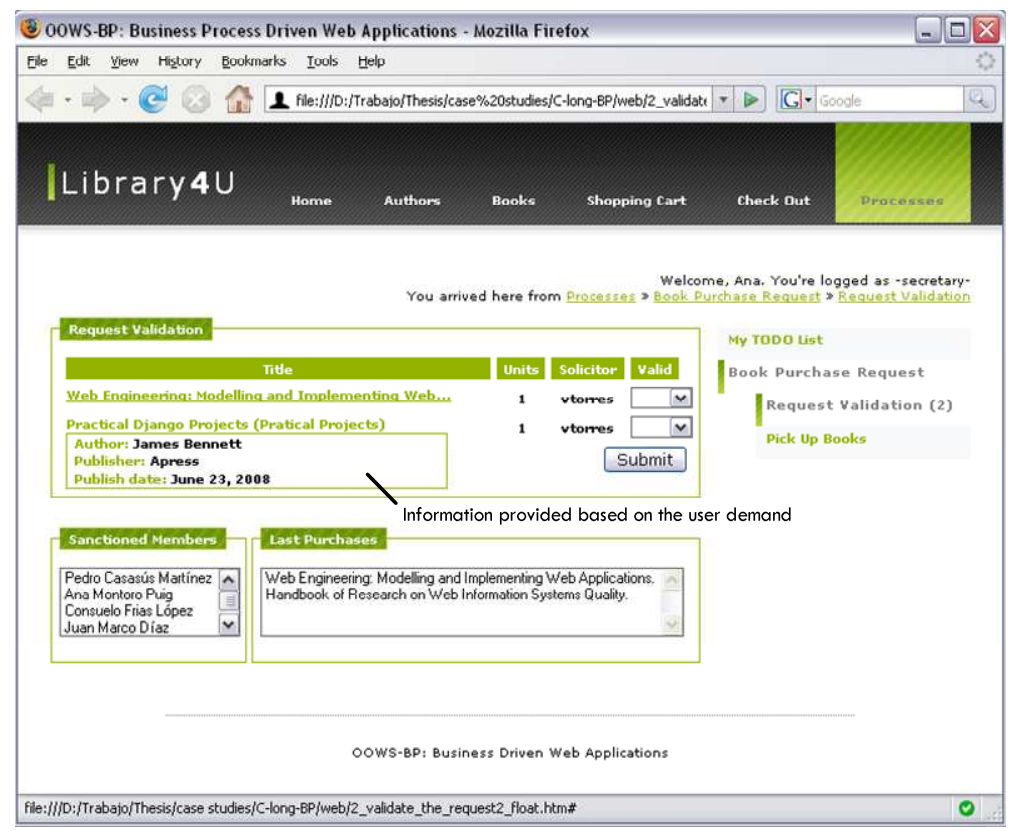

Figure 7.2 Details on Demand Pattern in use

A generic diagram of this primitive is shown in Figure 7.3. As this figure shows, a Detail On Demand pattern is applied to the index Navigational primitive. This primitive is depicted as a white rectangle containing two different sections. On the one hand, the anchor section specifies which attribute is going to be used to retrieve the "hidden" data. The available attributes for this section corresponds to the attributes specified in the attributes section of the index primitive. On the other hand, the detail section specifies which data is going to be shown in the new floating widget. In this case, the attributes included in this section are limited to the ones 
retrieved by the class-views and services-data-views defined within the Main-AIU.

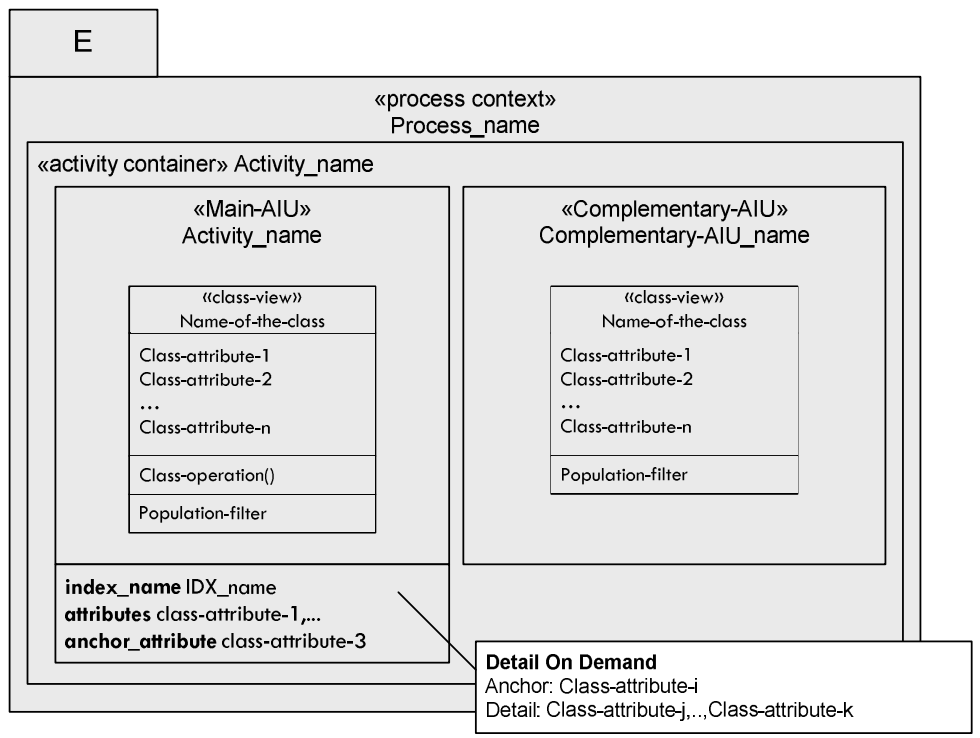

Figure 7.3 Detail On Demand Pattern

Abstract Syntax: This pattern allows specifying how the data contained in a Web page is shown to the user based on the user demand. This pattern is associated to the AIU primitive and can only include attributes from the views included in the corresponding AIU. A complete specification of this pattern includes an (1) anchor that could be used to retrieve the hidden information and (2) the set of information that is going to be displayed in the floating widget.

Figure 7.4 shows an excerpt of the Presentation metamodel including the Details On Demand primitive. This primitive defines a specific type of layout, and that is why this has been defined as a subclass of the Layout primitive. In addition, we have already 
mentioned that this primitive can only be associated to indexes defined within an AIU. This is shown by means of the relationship between the Details On Demand primitive and the index primitive defined in the Navigational Metamodel.

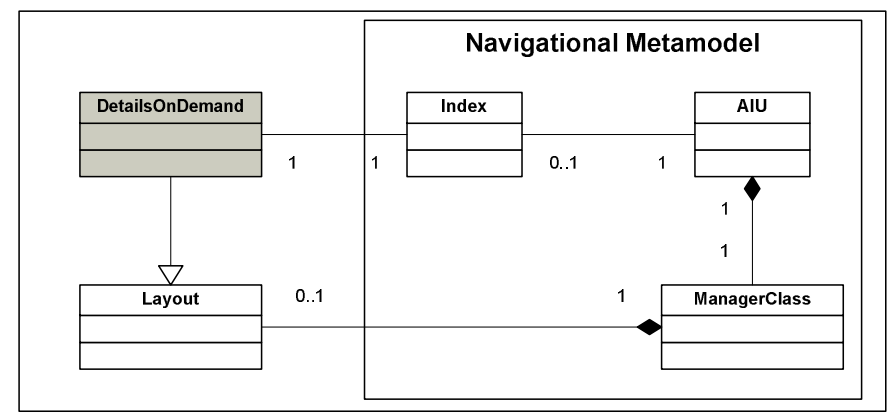

Figure 7.4 Metamodel Excerpt including the Details on Demand primitive

\subsubsection{List Builder Pattern}

Problem: The user is presented with a list of items which correspond to different instances of the same BP activity. In this case, the user needs to manage this list easily and quickly.

Concrete Syntax: According to Figure 7.1, the "Validate Request" Main-AIU has been associated with the "List Builder" pattern. The application of this pattern allows us executing the functionality associated to the corresponding $\mathrm{BP}$ activity (in this case the "validateRequest() operation) to all the instances retrieved by the Main-AIU.

Figure 7.1 shows how this pattern is specified at the modelling level. It can only be applied to AIU primitives. In general, this pattern can be applied to any list whose item lines accept the same 
functionality. For instance, in the context of a BP execution, this pattern can be used for completing multiple instances of the same process task.

Figure 7.5 shows the application of this pattern to the "ValidateRequest" Main-AIU. This pattern allows users speeding up the completion of tasks and processes. However, this pattern should only be used with operations requiring up to one argument. On the contrary, the interface would get too much complex.

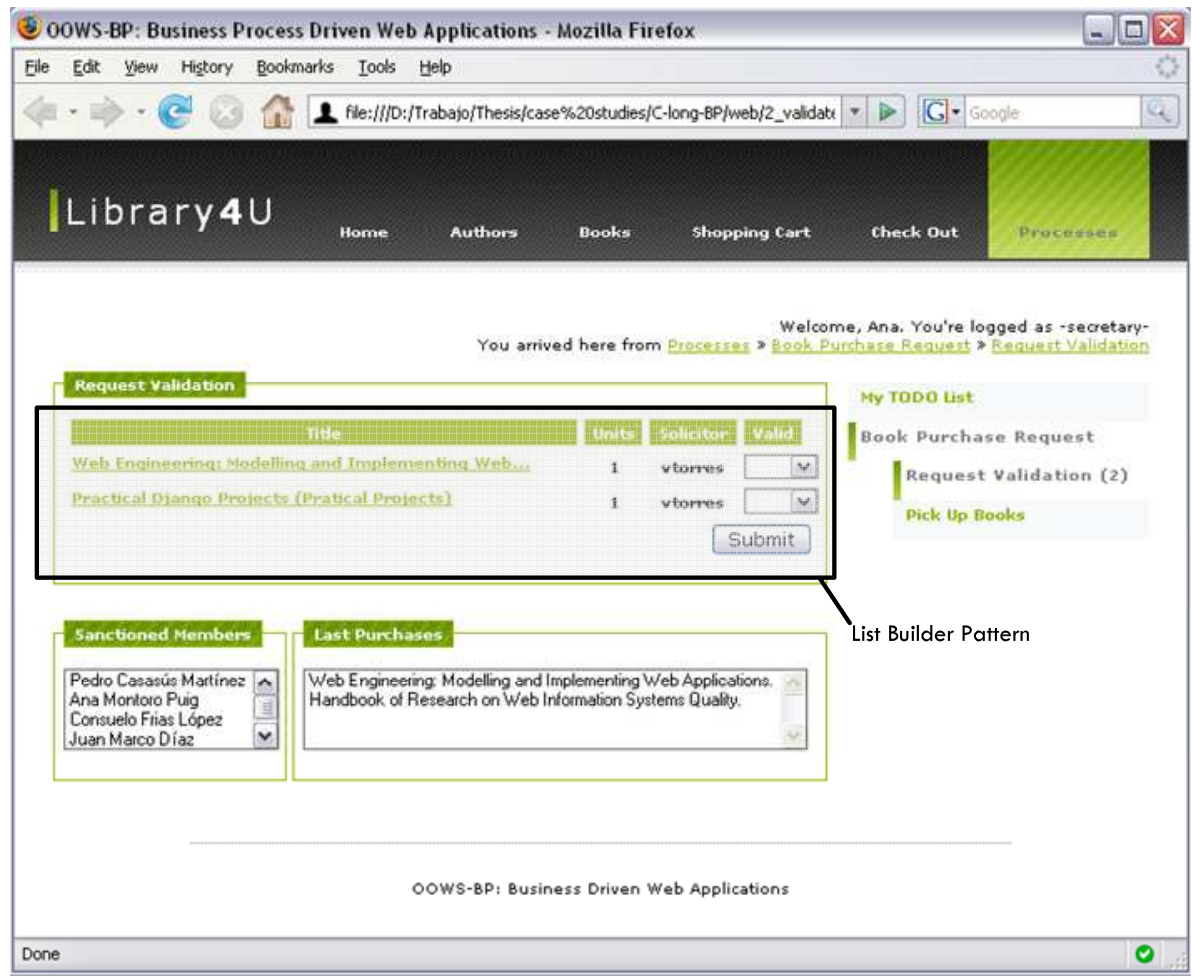

Figure 7.5 List Builder Pattern in use

Abstract Syntax: This pattern is used to allow the user dealing more efficiently with the data retrieved by the GUI. It allows 
users building up and managing a list of items at the same time. In this case, the selected functionality can be applied on many items at the same time.

Figure 7.6 shows an excerpt of the Presentation metamodel including the List Builder primitive. This primitive is associated to the Main-AIU primitive defined in the Navigational metamodel. As the multiplicity of the composition relationships states, the association of this pattern is optional.

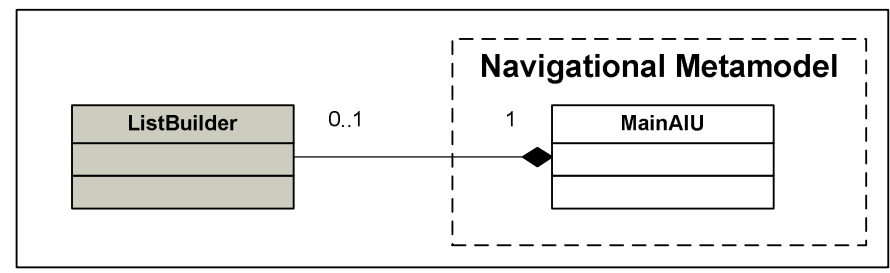

Figure 7.6 Metamodel Excerpt including the List Builder primitive

\subsection{Extended Presentation Metamodel}

This section presents the primitives presented in the previous section (those depicted in grey-colour) but this time integrated with the original ones. In addition, since Presentation primitives are defined over the primitives defined in the Navigational metamodel, the related primitives from the Navigational model have also been included. 


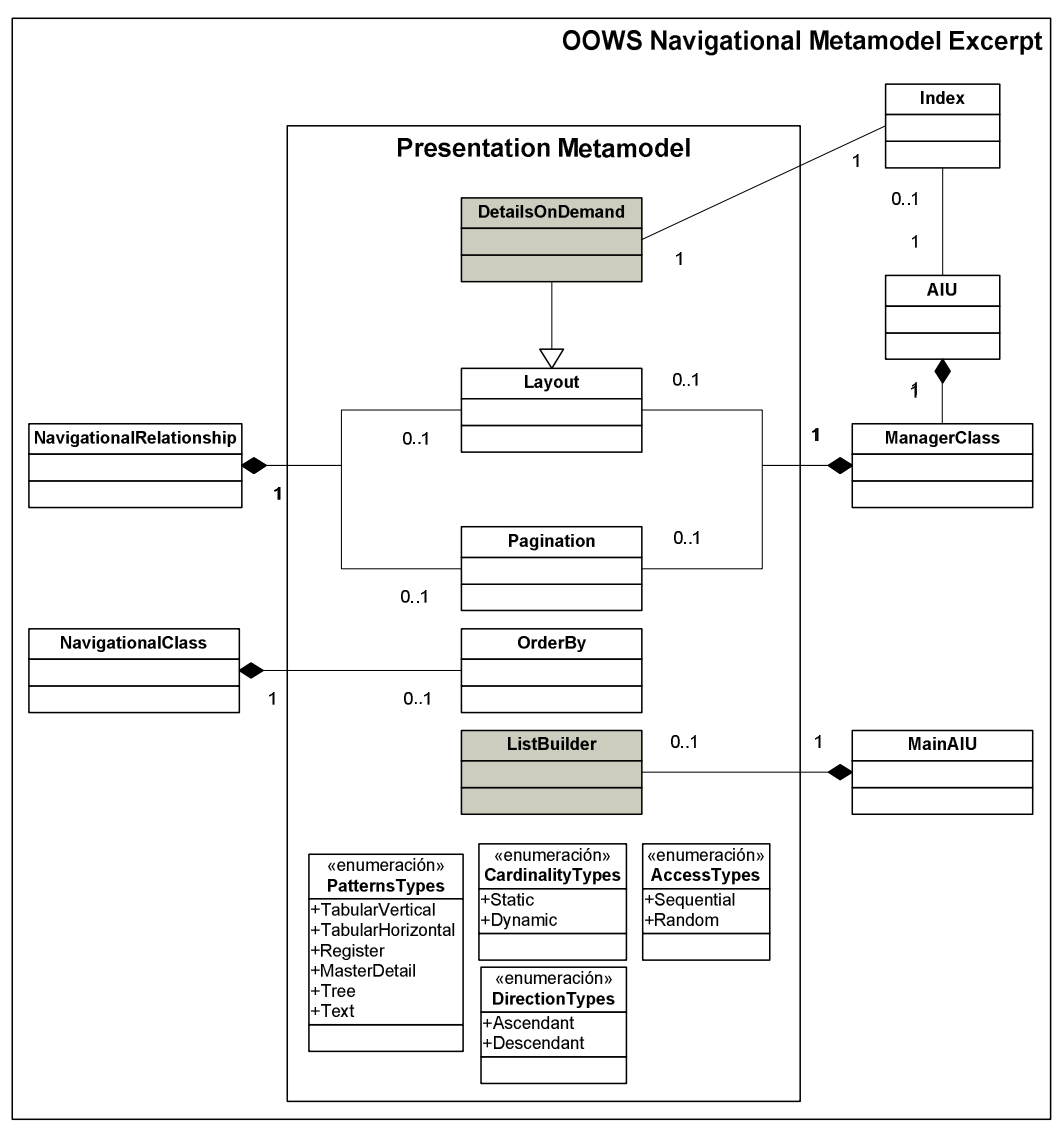

Figure 7.7 Presentation Metamodel

\subsection{Dealing with Usability Issues}

Up to now, we have presented the presentation patterns that have been defined in the OOWS proposal to deal with presentation issues. However, in order to ensure the quality (understanding this term as user satisfaction) of the generated user interfaces, we have also considered a set of guidelines proposed by usability experts. 
In most cases, the success of Web systems relies on the user acceptance, which is associated to the user experience. In the usability research area, the community has produced guidelines and standards that face the different usability problems introduced by web applications (Nielsen, 1999), (Fowler, 1998), (van Welie), (Mayhew, 1992). However, in order to ensure that these guidelines are used and applied properly, it is necessary to integrate them during the software development process and provide them with tool support (Abran et al., 2003).

If we concentrate just on the problems that can arise when performing a business process in a web application we find that usability guidelines are oriented to ensure that users understand correctly the data and functionality that is shown to her. Therefore, the usability issues that are going to face up in this work are the following:

- Preventing Input Errors

- Notify Users when they are Expected to Perform a Task

- Displaying the Tasks Pending to Complete (Wizard pattern)

The following subsections present how the OOWS approach cope with these usability guidelines. The following subsections have been organized in two parts, first we state the usability problem being considered as well as the kind of processes in which this usability problem applies and then we present the solution designed in the OOWS approach to be compliance with the corresponding guideline. 


\subsubsection{Preventing Input Errors}

Users are required to input some data (usually by means of a web form) and they are not provided with enough information regarding the kind of data they are expected to input. As a result, web forms are sent with data provided in incorrect formats and/or unfilled fields.

This problem refers to usability guidelines gathered in (van Welie), (Mayhew, 1992) and it can appear in both short and longlived processes. The guidelines provided by experts are summarized in the following list:

- Provide right field length allowing users to see their entered data.

- Partition long data items to aid users in detecting and reducing entry errors.

- Minimize user data entry filling input elements providing default values.

- Provide examples of input data that help users understanding the information that they are asked to input.

- Do not make mandatory fields whose data is not required to continue the transaction.

- Use informative error messages.

Solution. These guidelines have been handled either at the Structural Model and the Presentation Model as follows:

Class attributes and operation's parameter defined in the Structural Model include among other properties the followings: 
- Data type: It let us define the type of the attribute.

- Is mandatory: It allows specifying weather this attribute is required to execute the operation.

- Valid value range: It let us define and control which values can be entered.

- Default value: It lets the system suggest a predefined value to the user. The default value is a well-formed formula that is type-compatible with the attribute.

In the Presentation Model we associate to the Navigational attributes included the following properties:

- Alias: It allows defining an alternative name to the property being defined in order to present it more clearly to the user. (Ex. An attribute whose name is tel_number and its alias is Phone Number).

- Edit mask: It determines the format that the data shows when the user enters it. This mask is used for several purposes, (1) it is used to show the user an example of the data that she is expected to input, (2) it is used to partition long data items following the mask format and (3) it allows to provide a correct length of the input element.

- Help message: It allows specifying a text that can be shown to the user to provide some information about the meaning of the data to be entered.

- Validation message: is a piece of text that is shown to the user when a validation error occurs on the entered data.

With all this information defined at the modelling level we can generate graphical user interfaces that satisfy the above 
mentioned usability guidelines and that help the user understanding the data that she is asked to introduce.

On the other hand, other usability guidelines gathered in (Nielsen, 1999; van Welie) are better applied directly in the transformation rules that generate the corresponding code. The main reason to move them directly into the transformational level is that they refer to design issues more than modeling issues. Examples of guidelines of this type are:

- Using the most appropriate input element (radio buttons, checkbox, list box, etc.) regarding the kind of data being handled.

- Marking mandatory fields clearly.

- Check syntactic errors at the client side (avoiding sending incorrect data to the server, what implies lengthening the time dedicated to complete a task).

These three guidelines are introduced in the rules as follows:

- Appropriate widgets for input elements are generated following the guidelines provided by Jackob Nielsen in his Alertbox column ${ }^{24}$ in conjunction with the form pattern defined by (van Welie). These guidelines advice the most appropriate widget depending on the data being represented (number of different options, exclusion, multiple selection, etc.)

- Labels of mandatory input fields are attached with the asterisk symbol (*).

${ }^{24} \mathrm{http} / / / \mathrm{www} \cdot$ useit.com/alertbox/20040927.html 
- Client-side scripting languages (such as JavaScript or VBScript) or web development techniques (as AJAX ${ }^{25}$ ) are generated to check syntactic errors at the client side.

\subsubsection{Displaying the Tasks Pending to Complete}

When a user starts a short-running BP it is better to display the user with the set of steps involved in the process. This information can be used by the user to have an idea about the information that she has to provide in order to complete the process. On the contrary, regarding long-running $\mathrm{BP}$, the user should only be displayed with the next pending task and not with the whole list of tasks to complete the process. Moreover, in this case, it is not important to provide the user with information about the steps that she has already perform in the process.

Solution. The GUI should include (just for short-running BP) an area that displays the sequence of tasks/steps that need to be performed in order to complete the process. Moreover, the current task being performed by the user must be distinguished among the rest of the tasks. This area can be generated from the BP definition. Figure 7.8 depicts graphically the models involved in the transformation process to obtain the final web pages. As this figure shows, only those activities defined in a $\mathrm{BP}$ that require interaction with the user are first transformed into Activity Containers in the Navigational Model (NM), which are later on

\footnotetext{
25 http://www.adaptivepath.com/publications/essays/archives/000385.php
} 
transformed into the web pages that will provide support to each of those activities.

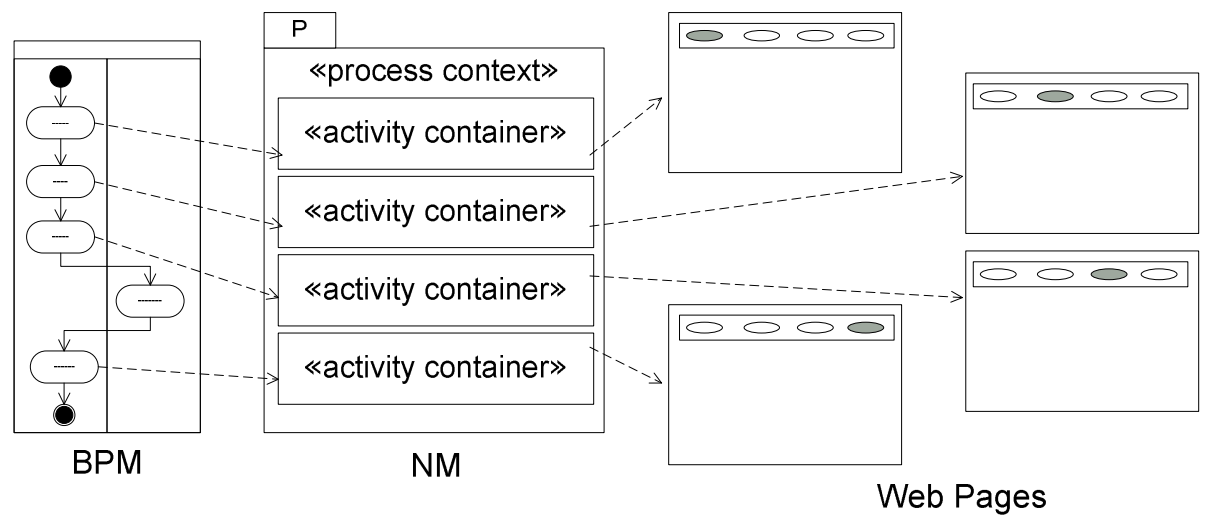

Figure 7.8 Models Used for the generation of Web Pages

The generated web pages include an area where the user can see the list of activities that constitute the process being executed (see group labelled as A in Figure 7.9). Moreover, the current activity is highlighted to help the user identify clearly the current action being performed (see group labelled as $\mathrm{C}$ in Figure 7.9). 


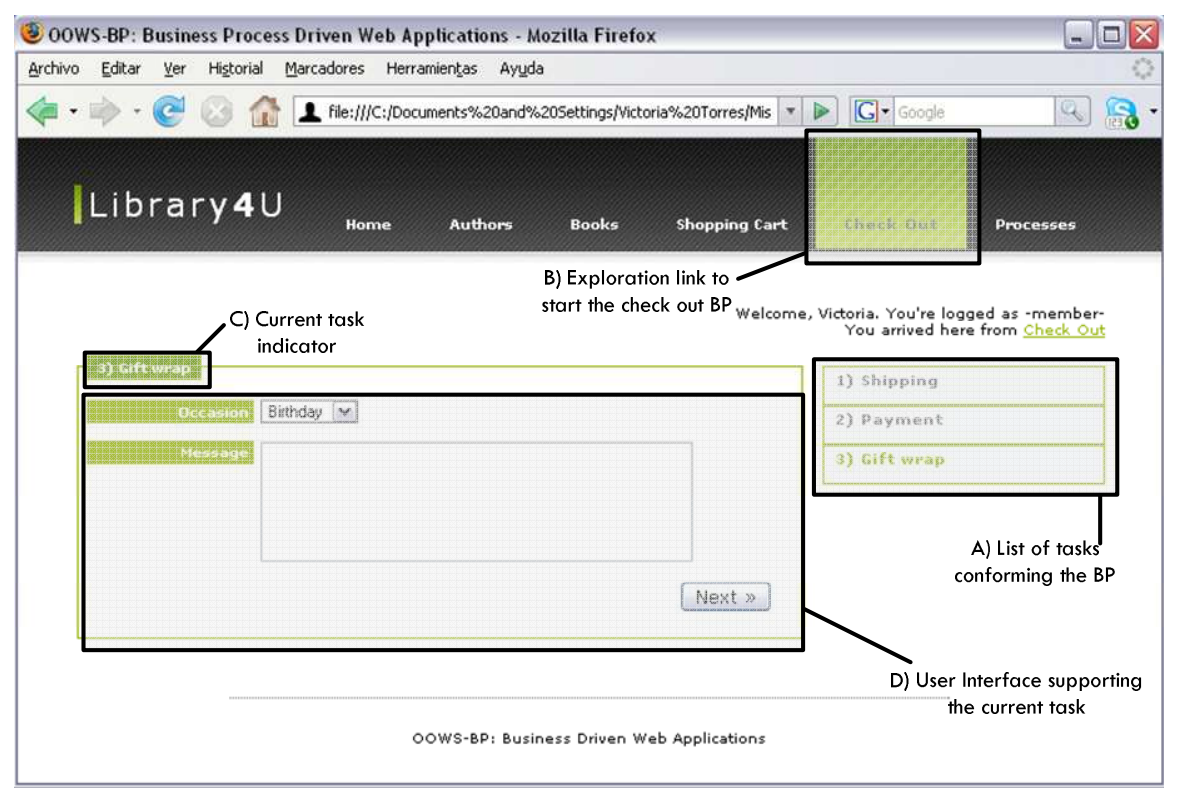

Figure 7.9 Generated User Interface for a Short-running BP

\subsubsection{Notify Users when they are expected to perform a Task}

During the execution of a long-running BP many different participants can take part in it. After the completion of an intermediate task new task(s) turns into pending tasks for the same role or for a different one. However, if these new pending tasks require participation of a human being different from the one completing the previous task, she will not perform her job until she checks if there is any pending activity in their TODO list what can derive in a longer delay of the process.

Solution. To minimize this problem, we have extended longrunning BPs with tasks that notify users when they become 
responsible of a new activity. These new tasks are added before any activity requiring human participation. However, these new tasks are only added when the process flow moves to another role associated with a human being different from the one performing the previous task.

Following the solution mentioned previously, we generate an extended version for the Request Book Purchase BP as shown in Figure 7.10. The new grey-coloured added tasks refer to services that send alerts to the involved users in order to make them aware of these new tasks. By default the process sends alert mechanism via email messages, however, other mechanisms such as Short Message System (SMS) or voice messages could also be considered.

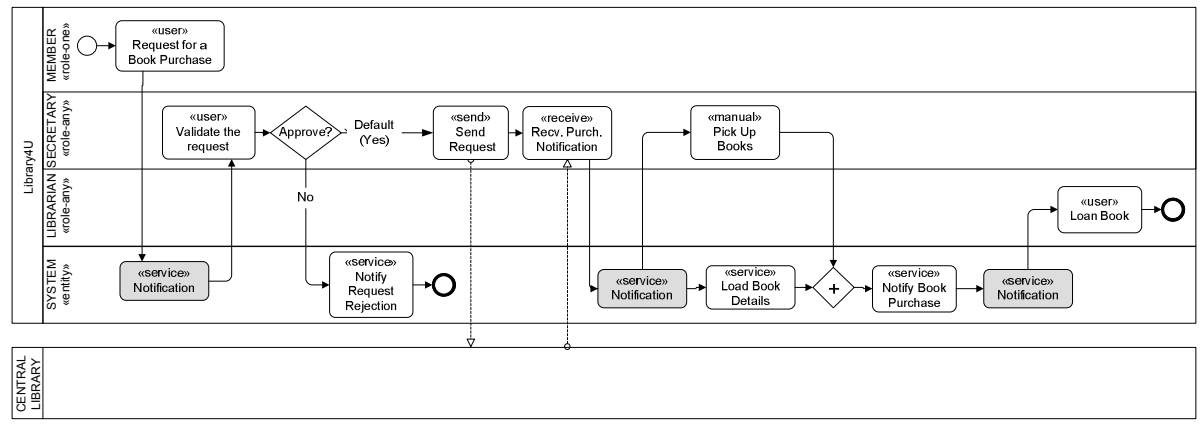

Figure 7.10 Modified version of a BP definition

\subsection{Conclusions}

This chapter has presented the extensions defined to the presentation model to deal with the execution of BPs. The presented extensions have been introduced to improve the user experience. As a result, users can perform process tasks more 
efficiently and faster. This is possible since the user is display with useful information about the kind of task being performed and the kind of information she is asked for. On the one hand, the Details On Demand pattern allows the user getting more information just in case the user really needs it. Therefore, the use of this pattern avoid overloading user interfaces what results in more clear interfaces. On the other hand, the List Builder pattern allows accelerating the completion of multiple task instances.

Moreover, to improve the usability of the generated web interfaces we have also taken into account the guidelines proposed by the expertise community in web design. To deal with these guidelines we have considered them, depending on its suitability, either in the OOWS models or directly into the model to code transformations. 


\section{Chapter 8}

\section{Architectural Extension}

This chapter presents the extension defined at the architectural level for Web applications to deal with the execution of Business Processes (BPs). This extension has been designed trying to keep the separation of concerns in which the OOWS method has been conceived. Therefore, to keep separate the navigation that happens during pure navigation and BP execution, we have introduced a process engine into the architectural tier. This element allows us obviate the navigation within the navigational model since this is going to be fully controlled by the process. As a result, a more clear and easy to handle navigational model is built for $\mathrm{BP}$ execution. In addition, as a consequence of introducing a process engine into the architecture, process definitions have to be defined in a process executable language. In particular, in this work we have chosen the WS-BPEL language. This language allows specifying BPs behaviour based exclusively on Web Services. This limitation forced us to introduce new elements into the business layer to handle the lack of WS-BPEL for supporting human tasks. 


\subsection{Introduction}

Similar to the introduction of database management systems into the architecture of software systems, dealing with systems that support the execution of Business Processes (BPs) requires the introduction of solutions that allow us handling BPs properly. These solutions provide mechanisms to model, automate, integrate, monitor and optimize BPs continuously (Hollingsworth, 1995). From this set of mechanisms, the one we are interested of at this point is the one related with the automation of BPs. This automation is achieved by the use of a process engine which launches and run instances of existing BPs.

Based on the decision of adopting WS-BPEL as the executable language of processes (see chapter 2), we have introduced into the architecture of the generated Web applications a process engine that implements this specification. In particular, we have made use of the ActiveBPEL ${ }^{26}$ process engine.

However, as WS-BPEL is based on Web services, when we want to model workflows (processes that include human participants) we have to make use of some mechanisms on top of the original specification to allow us handling the asynchrony introduced by this kind of process participants ${ }^{27}$.

\footnotetext{
${ }^{26} \mathrm{http}: / / \mathrm{www}$.active-endpoints.com/active-bpel-engine-overview.htm

27 Trying to face this limitation, the WS-BPEL Extension for People (Kloppmann et al., 2005) has been proposed.
} 
This chapter presents the extension defined at the architectural level in order to cope properly with the requirements of the kind of Web applications that we are dealing with.

\subsection{Extension Overview}

In this section we present the whole picture of the architecture of the kind of Web applications we are dealing with. As Figure 8.1 shows, the architecture follows the classical 3-Tier architecture. In this architecture each layer interacts only with the layers placed immediately next to it and is responsible for a specific function. From these three layers, we are going to focus on the Business layer, layer where the presented extension has been placed.

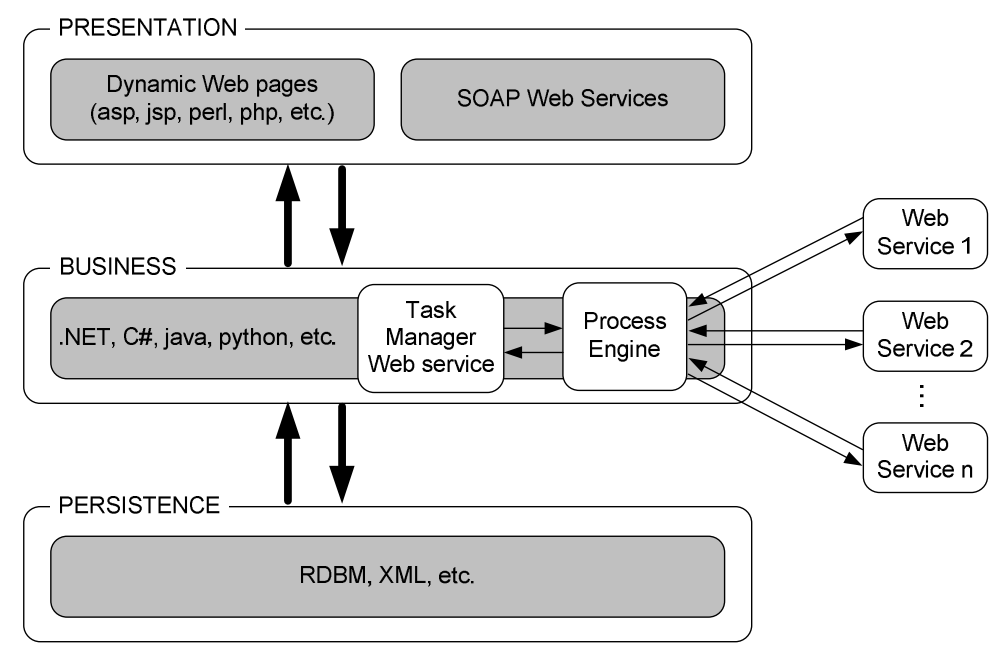

Figure 8.1 Three Layer architecture for Process-driven Web applications

In order to keep the separation of concerns established by this three-architecture tier, the Model-View-Controller (MVC) architectural pattern (Reenskaug, 1979; Reenskaug, 2003) is the most appropriate solution to be applied. Each part of the pattern 
is in charge of a specific task, Models are used to maintain data, Views are used for displaying all or part of the data and finally Controllers are used for handling events that affect the model or view $(\mathrm{s})$. The major benefits of this separation of concerns are (1) the reduction of the complexity of the architectural design and (2) the increase of flexibility and reuse. On the one hand, complexity is reduced since the controller part decouples data access and business logic from data presentation and user interaction. On the other hand, the increase of flexibility and reuse is achieved by the possibility of defining multiple views for the same model depending on the target user.

In this work, the proposed architecture has been mapped to the Tapestry Web Framework ${ }^{28}$. In this framework, the different parts of the MVC pattern are represented by different types of files as Figure 8.2 shows.

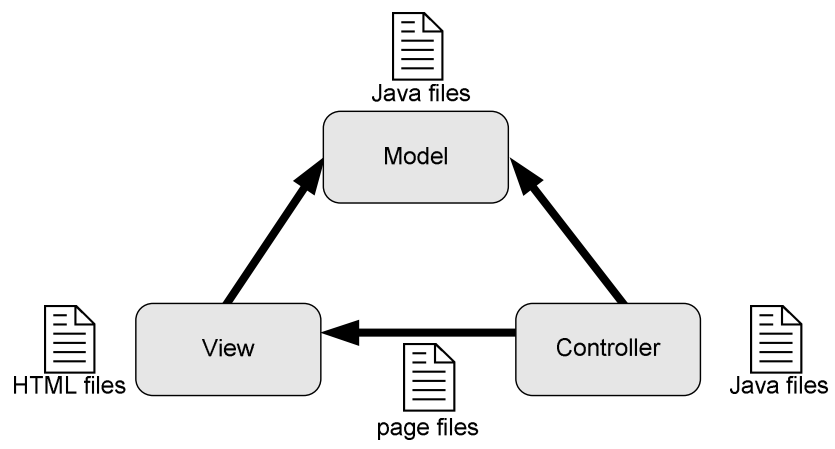

Figure 8.2 Tapestry File Organization

$28 \mathrm{http}: / /$ tapestry.apache.org/ 
Java files are used to represent the Model and Controller part of the MVC pattern. On the one hand, Java files representing the Model part define the domain logic of the application. On the other hand, Java files representing the Controller part define the behaviour of the View part of the pattern. Code excerpt 8.1 shows part of the Java file associated to the Home.html page. This Java file contains the code that completes the corresponding static HTML template with dynamic data.

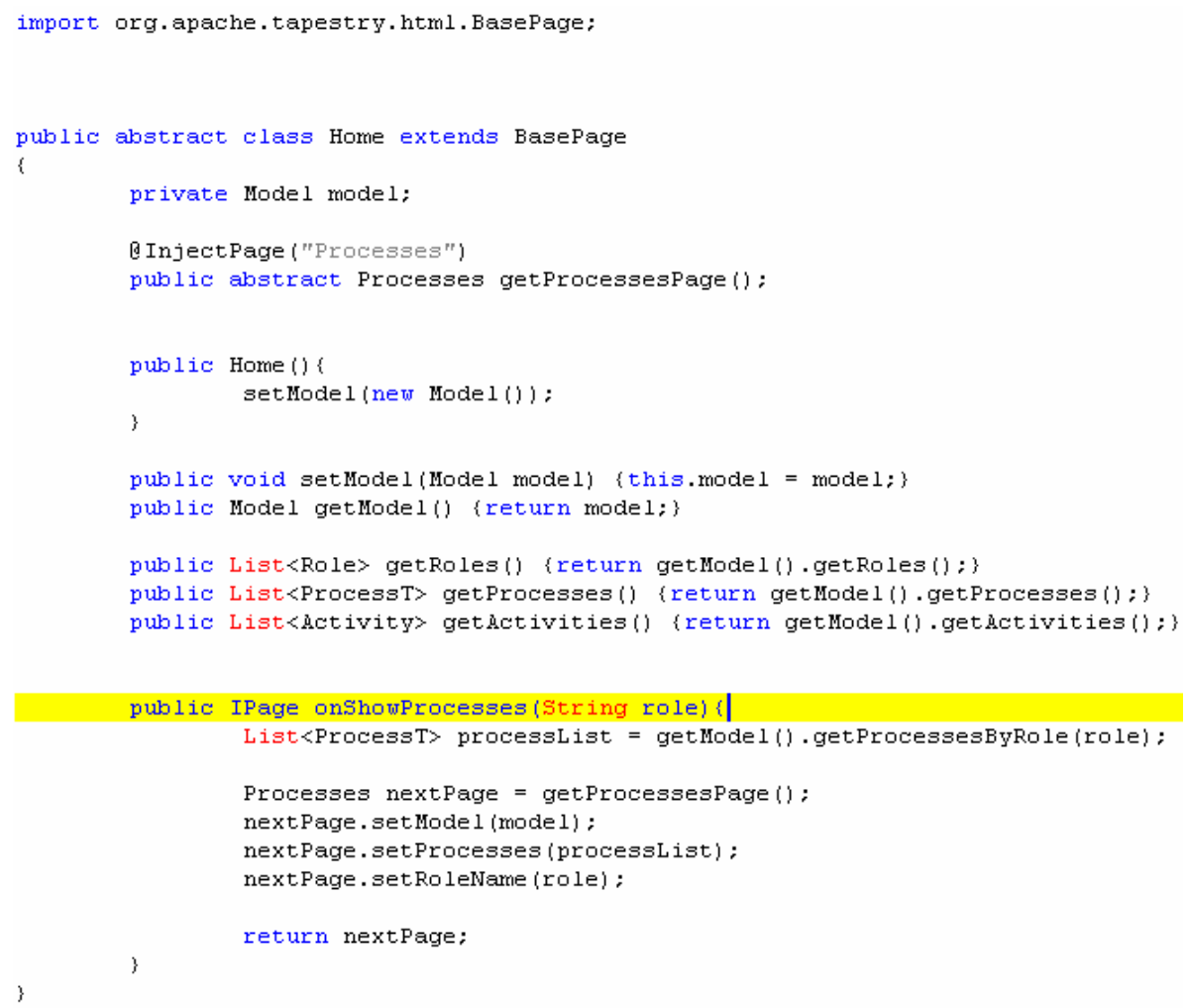

Code excerpt 8.1 Java file completing the corresponding HTML template

HTML files are used to represent the View part of the pattern. These files are HTML templates using pure HTML which includes place holders for Tapestry components. Some of the built-in 
Tapestry components are For (to loop over a collection of source values), Insert (to allow for the insertion of text into the HTML response), DirectLink (to create an $<\mathrm{a}>$ hyperlink that notifies the component when the link is triggered) or Submit (to provide an HTML form submission element). Code excerpt 8.2 shows the HTML template built for the Home page. Within the HTML code and associated to the span tag we find the "jwcid" special attribute which references to a Tapestry component.

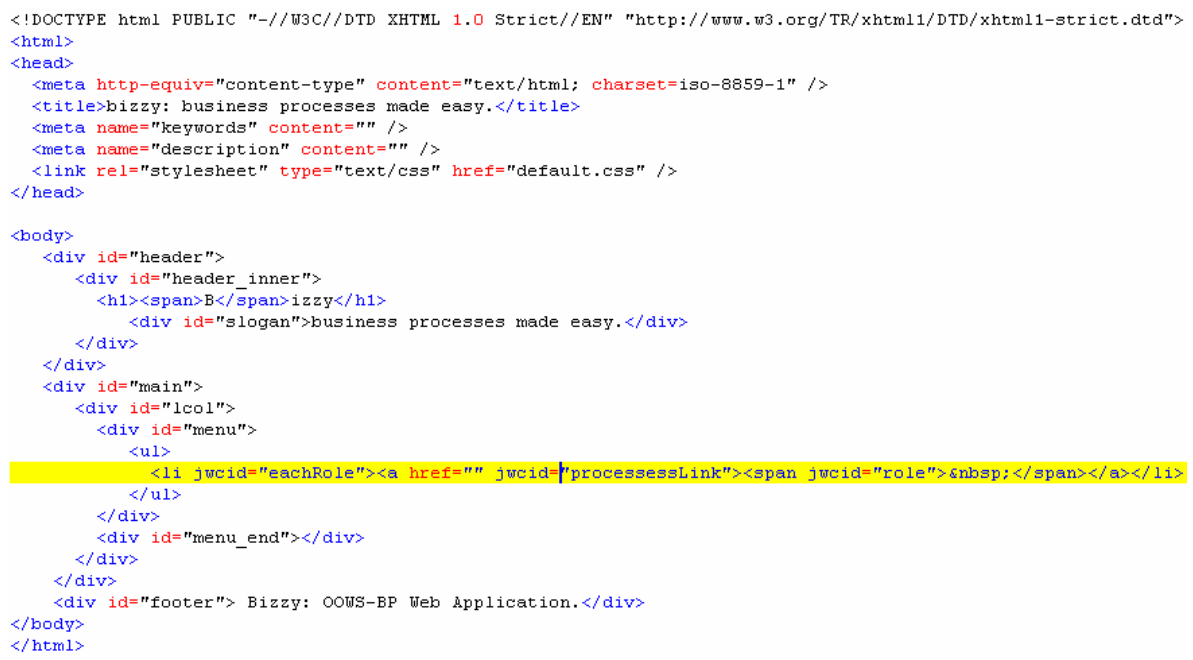

Code excerpt 8.2 HTML Template

Page files are XML descriptor files that allow combining HTML templates with Java code. These files are optional but they contribute to have a more legible code. Code excerpt 8.3 shows the XML included in the Home.page descriptor. This file links HTML templates with the associated Java files. 


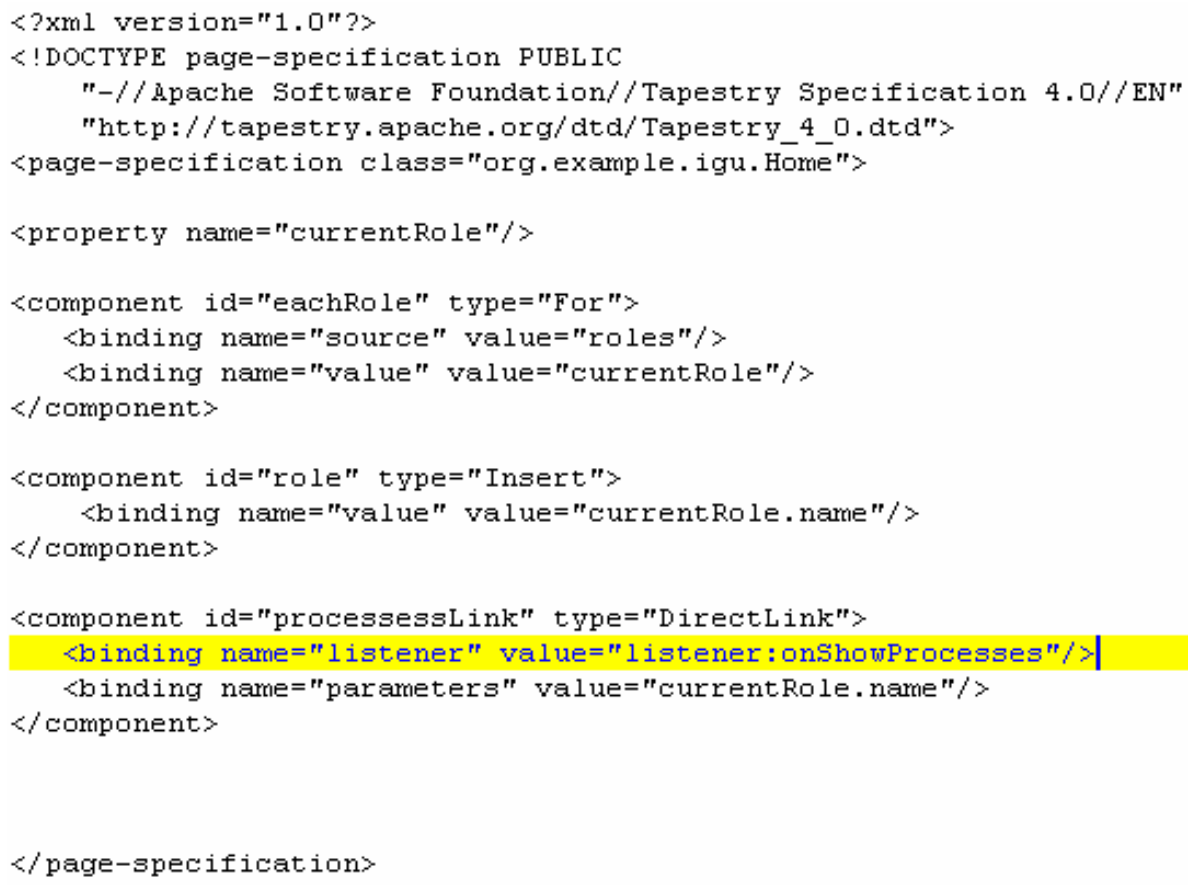

Code excerpt 8.3 XML Descriptor

\subsubsection{Presentation Layer}

The Presentation Layer is the layer responsible for (1) providing an interface for the end users into our application and (2) for exposing business functionality to external partners by means of Web services. In Web applications, for the generation of graphical user interfaces, this layer includes dynamic web pages which are created by server-side languages such as ASP, JSP, PERL, or PHP among others. On the other hand, the business logic exposed to external partners is provided by means of Web services. 


\subsubsection{Business Tier}

This tier communicates with the presentation layer and the data layer and depending on the case, its responsibility changes. On the one hand, the Business Tier is responsible for accessing the data tier to process (retrieve, modify and delete) data to and from the data tier. On the other hand, it is responsible for sending the results to the presentation tier.

The extension proposed in this chapter involves this tier. This extension includes two new elements which are the Process Engine and the Task Manager Web Service. The function of the Process Engine is creating and running new process instances from input WS-BPEL processes when an incoming message triggers the start process activity. Moreover, since WS-BPEL is based on Web services, and these can be hosted on different servers, the business tier can be distributed being their components linked by the process engine. The Task Manager Web Service (see Figure 8.3) is the element that comes into play when activities related to humans are invoked. This service takes the responsibility of handling this kind of activities, and communicates with the process engine and the application logic (a detailed explanation is provided later on in the chapter). 


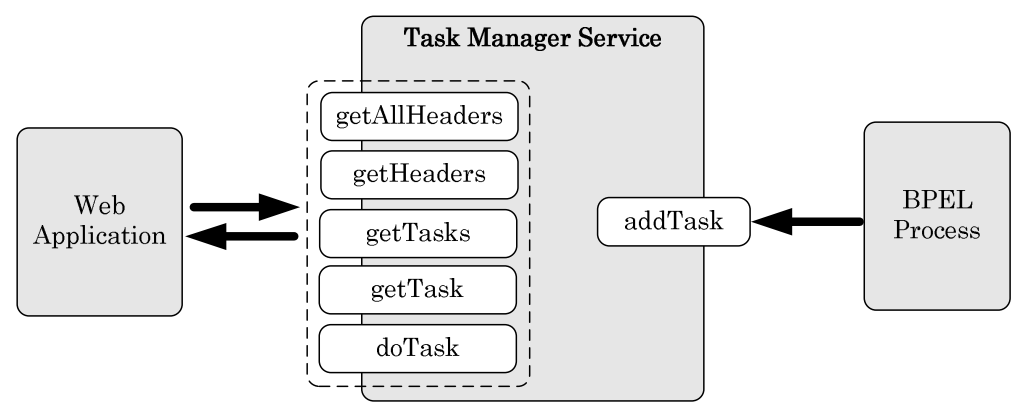

Figure 8.3 Task Manager Service Interface

\subsubsection{Data Tier}

The data tier is intended to deal with the storage and retrieval of information (no business logic is placed here). In this tier we usually find a Database Management System (DBMS) (MySql, SQLServer, Oracle, etc.) or plain text files.

\subsection{Dealing with Human Tasks}

In this section we present on the one hand the design of the Task Manager Web Service (hereafter TM-WS). On the other hand we present the communication that happens between the process engine, the TM-WS and the Web application when a human participant is involved in the execution of a BP.

\subsubsection{Task Manager Web Service}

As we have shown in Figure 8.3, the Task Manager Service exposes six operations to interact with both the WS-BPEL process and the Web application. A graphical representation of the WSDL file of this service is shown in Figure 8.4. 


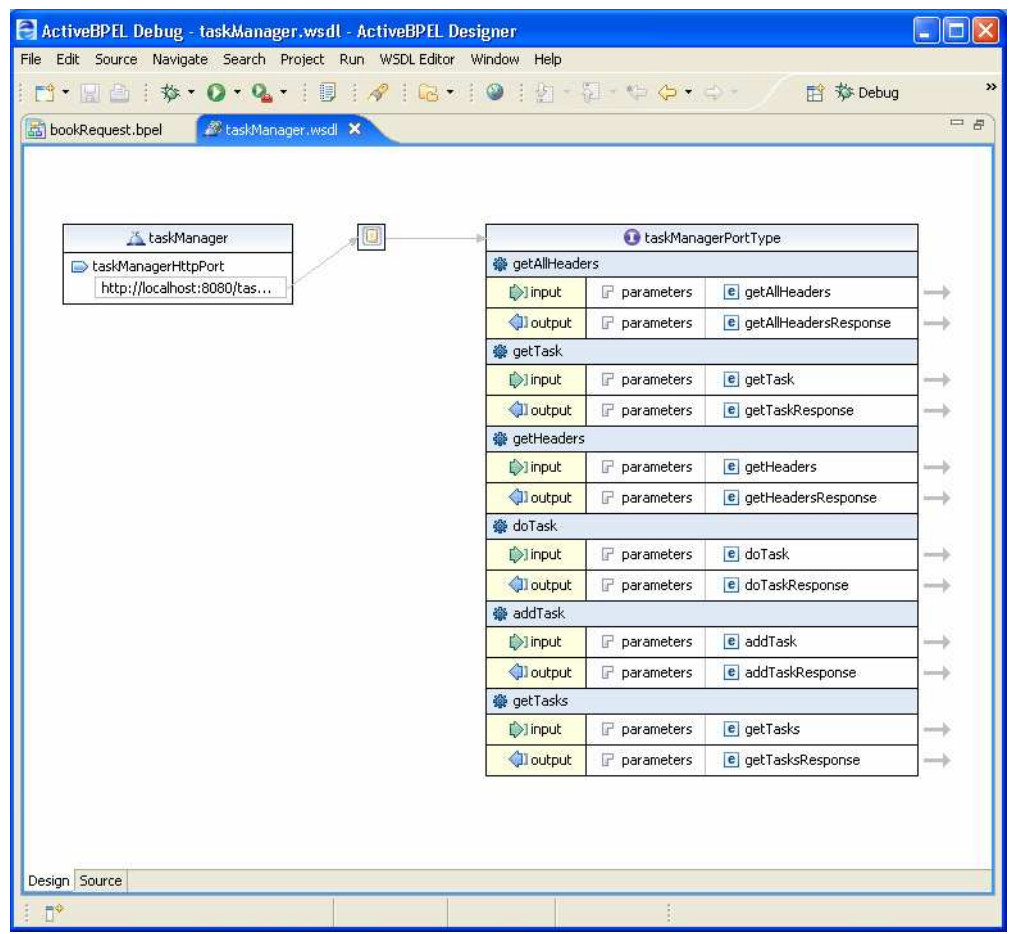

Figure 8.4 WSDL for the Task Manager Service

The interface of the TM-WS defines all these operations based on the way in which this has been implemented. To understand better these operations it is necessary first to explain how tasks are handled by this service. In Figure 8.5 we show the class model design to handle tasks. The TaskManager class handles zero or multiple tasks (relationship defined between this class and the Task class). Then, a task is defined by a header (composition relationship between the Task and Header classes) and a payload (document). On the one hand, the header includes information such as the identifier of the task, the user involved, the user group and the name of the process and the task. On the other hand, the payload is a XML file containing the data of the task. 


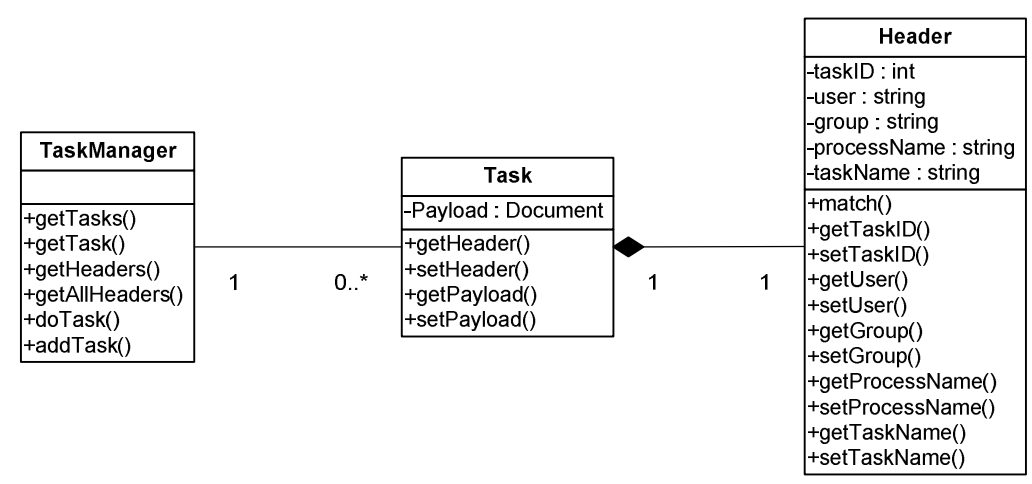

Figure 8.5 Task Manager Structure

Then, the operations published by the Task Manager are the following:

- "getAllHeaders" and "getHeaders" operations have been defined to access more efficiently to the information about the tasks kept by the Task manager. These operations will be invoked to build dynamically the menu that allows users to access their pending tasks. "getHeaders" accepts a query that allows specifying which tasks we are interested in (i.e. pending tasks for a particular user or for a group of users). The following Code excerpt 8.4 shows how the "getHeaders" operation is invoked by a particular function designed to see if there are pending activities for a specific process.

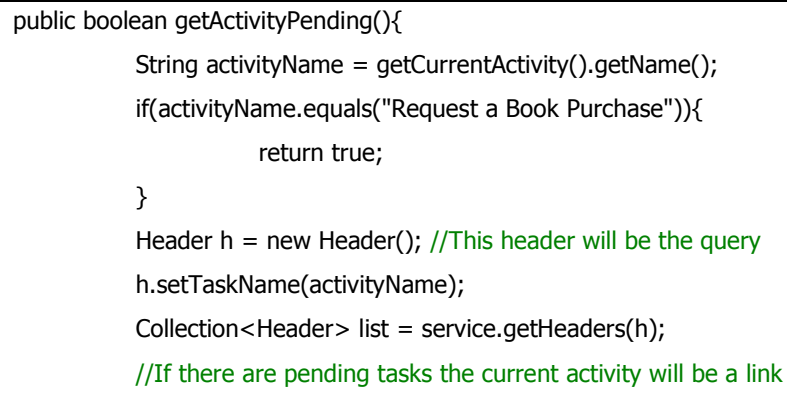


if(list.size ()$>0$ ) return true;

else return false;

\}

Code excerpt 8.4 Example of getHeaders operation invocation

- "getTasks" and "getTask" operations allow obtaining the tasks (differently from the previous presented operations that only retrieve the header part of the task) kept by the Task manager. "getTask" retrieves from a task identifier the whole task. Code excerpt 8.5 shows an example of invocation of the "getTasks" operation. In this case the getElements function invokes this operation to retrieve all the tasks instances corresponding to the "Validate the Request" task.

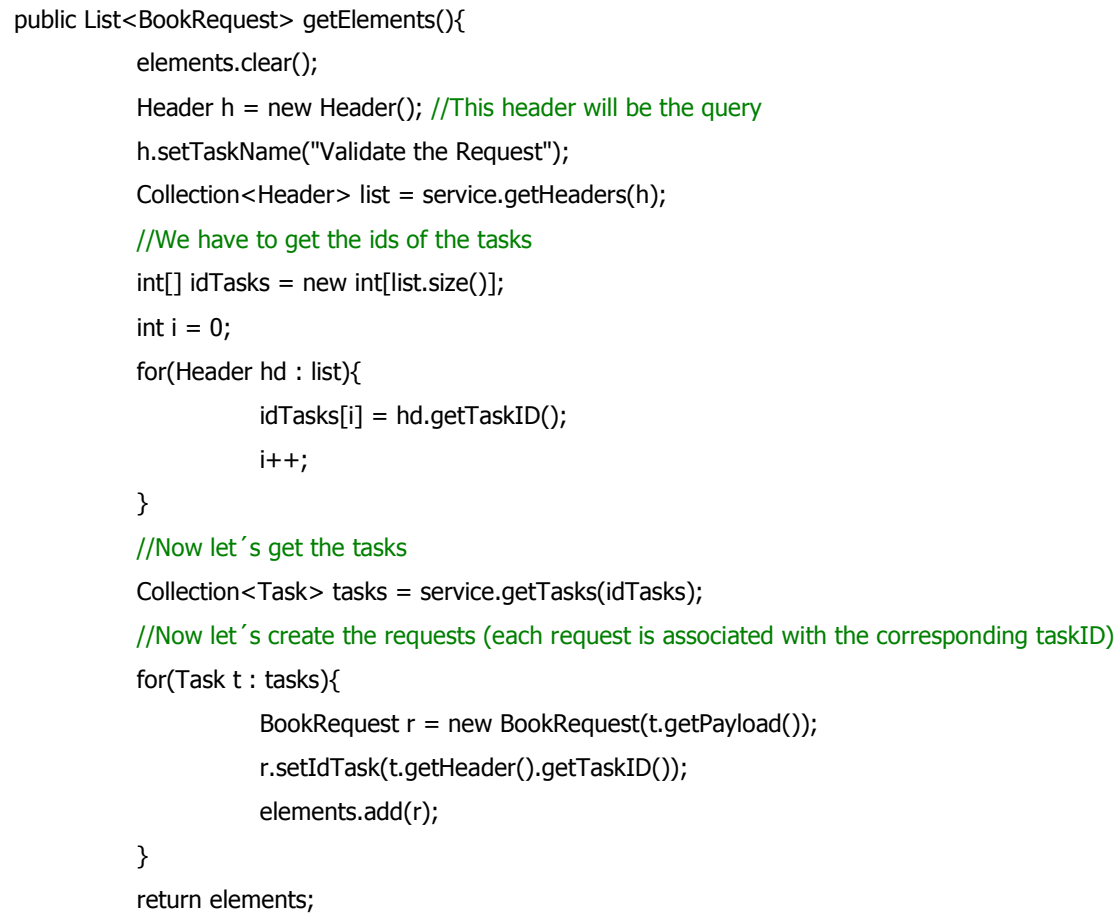


Code excerpt 8.5 Example of getTasks operation invocation

- "doTask" is the operation invoked by the application to notify the TM-WS for erasing the task whose identifier coincides with the one passed to the operation. The following code excerpt includes part of the generated Web application code where the "doTask" operation is invoked.

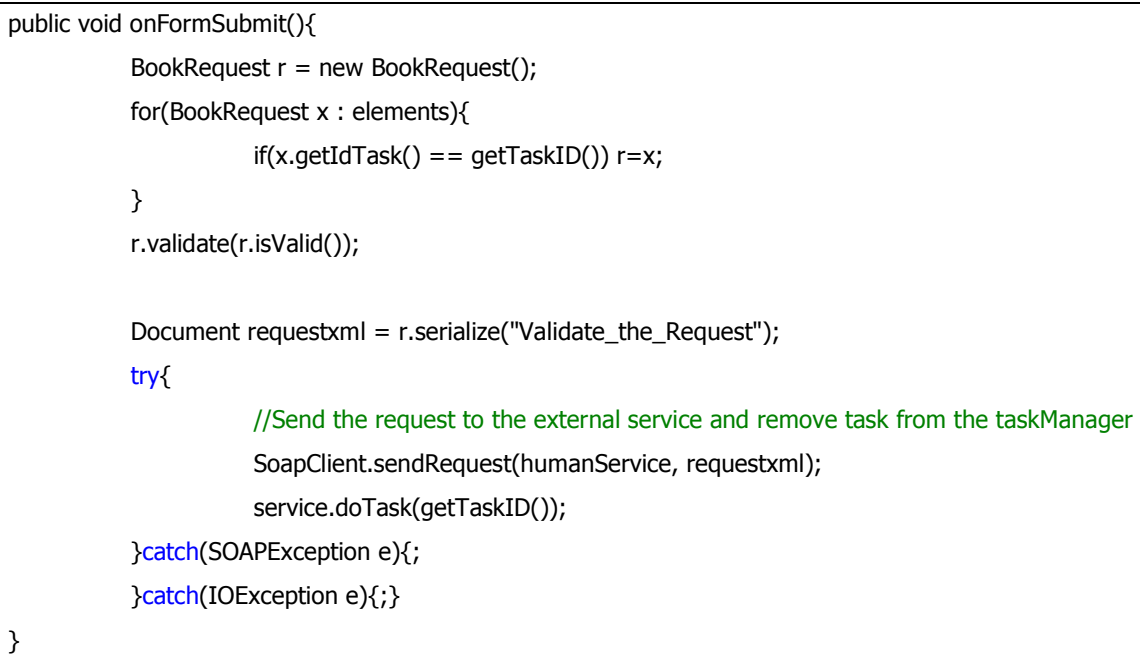

Code excerpt 8.6 Example of doTask operation invocation

- "addTask" is the operation invoked by the WS-BPEL process when a task involving human participation is reached by the process flow. As parameter, the operation accepts the identifier of the process instance. The reason for requiring this identifier is to perform the correlation between process instances and data. Code excerpt 8.7 shows WS-BPEL portion where the "addTask" operation is invoked. 


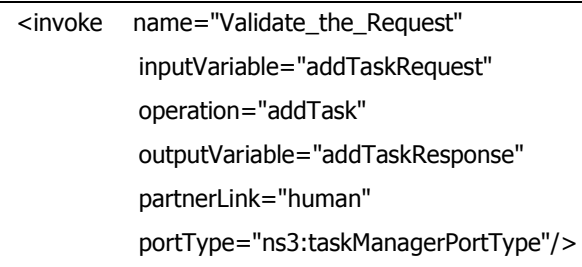

Code excerpt 8.7 addTask operation invocation from WS-BPEL code

\subsubsection{Web Service Conversation while Human Participation}

Once we have presented the interface exposed by the TM-WS, this section presents a scenario that shows the interaction accomplished between the WS-BPEL process, the TM-WS and the Web application when an activity requiring human participation is reached by the flow of the process. As Figure 8.6 shows, to represent this interaction we use UML Sequence Diagrams.

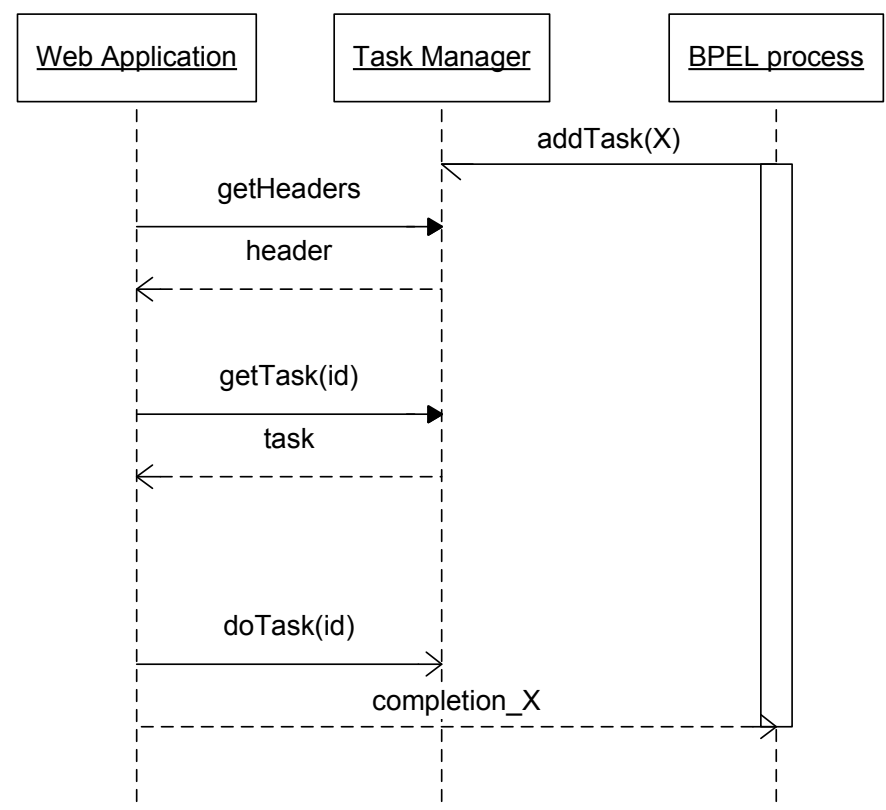

Figure 8.6 Role of the Task Manager Web service 
The invocation sequence is the following:

- First of all, the process engine starts running a BP (either short-running or long-running process) which includes some activities involving human participation (i.e. approve purchase, review code, etc). Then, when one of these activities is reached by the flow of the process, the WS-BPEL process sends a request to the TM-WS to handle it. This interaction is represented in Figure 8.6 by the asynchronous call invoking the "addTask" operation.

- Then, the TM-WS creates a work item to represent the request. Moreover, it stores correlation information so that a subsequent request to the WS-BPEL process can locate the originating process instance.

- The Web application, when a user logs into the system, contacts the TM-WS to reach the set of pending tasks for the logged user. At this point, the user is responsible for completing the work item, which is accomplished through the corresponding user interface from the Web application. The completion of the work triggers two calls. One the one hand, the "doTask" operation is invoked to notify the TM-WS that this task has been completed and can be removed from the Task manager. On the other hand, the "completion_X" operation is invoked to pass back the control to the WS-BPEL process (the "X" references the name of the task which coincides with the string passed as argument to the "addTask" operation). This operation is sent together with correlation 
information to inform the process engine about the completion of the task for a particular process instance.

\subsection{Conclusions}

This chapter has presented the extension performed to the architecture of the generated web applications in order to handle properly the execution of business processes. This extension involves on the one hand the introduction, at the business layer, of a process engine capable of handling BP instances. On the other hand, in order to cope with the lack of WS-BPEL to support processes involving human participants, we have defined a new service (the Task Manager Web Service) which handles this kind of tasks.

An important issue that we have taken into account is the portability of the provided solution. It was a must to keep WSBPEL process definitions independent of any process engine vendor. For this reason, the Task Manager Web Service has been defined as an external partner for the WS-BPEL process, similar to any other external partner. For instance, instance process correlation is achieved by means of identifiers generated by the "objects" handled in the own process instance. This decision allows us controlling correlation in the same way independently of the engine used. 


\section{Chapter 9}

\section{Model Transformations}

In previous chapters we have presented the necessary concepts for creating the models that allow represent properly BP-driven Web applications. However, to get the most from a Model Driven Development (MDD) approach as the one presented in this thesis, it is necessary to automate the transformations that allow translating models to other artefacts, including code. Depending on the type of generated artefact, a model-to-model (M2M) or model-to-text (M2T) transformation has been defined. Therefore, this chapter presents the set of transformations that implement the mappings between the models defined in the proposal and other artefacts such as models and code.

The chapter has been structured as follows. First of all, section 9.1 provides an overview of the M2M and M2T transformations defined along the development process. These transformations generate two different types of artefacts, which are an executable WS-BPEL process and a Web application. Then, according to the different generated artefacts, sections 9.2 and 9.3 present in detail 
the transformations implemented in each case. Finally, section 9.4 presents the conclusions of this chapter.

\subsection{The Big Picture}

Following the Model Driven Development (MDD) approach, we have defined and implemented a set of model transformations to obtain a complete Web application according to the architecture presented in chapter 8 . These transformations are dedicated to obtain (1) the WS-BPEL definition of the BPs modelled in the Business Process Model (BPM) and (2) the Web application that gives support to these BPs in a specific Web framework (the Tapestry Web Framework). Figure 9.1 and Figure 9.2 present graphically the set of steps that are necessary to obtain in each case the required artefacts. The numbers included in both figures define the order in which these transformations are performed.

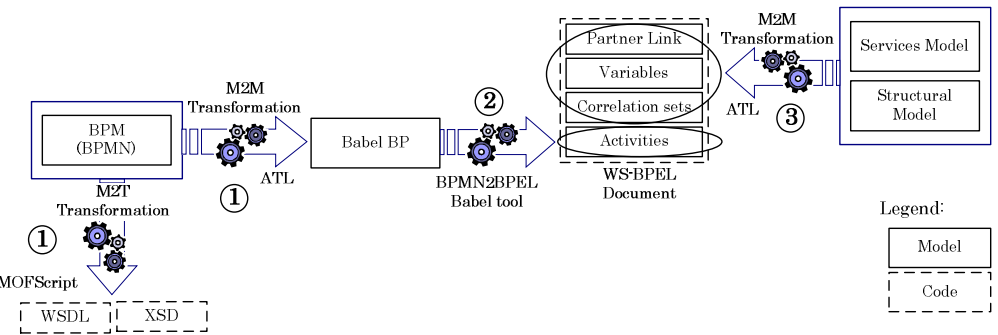

Figure 9.1 Transformations Defined for generating the WS-BPEL code

On the one hand, Figure 9.1 details the steps that have been defined to obtain, from a $\mathrm{BP}$ specification represented using the BPMN notation, an executable BP represented in the WS-BPEL language. The transformation process includes M2M and M2T transformations. In addition, the BPMN2BPEL Babel tool has 
been used to generate part of the WS-BPEL document. As this figure shows, this document is obtained not only from the BPM. It also requires the Structural and Services Models which are used to complete some of the WS-BPEL sections.

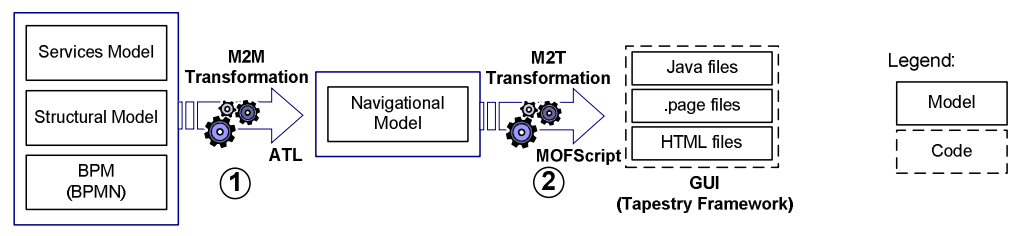

Figure 9.2 Transformations Defined for generating the Web site for the Tapestry Framework

On the other hand, Figure 9.2 details the steps that have been defined to obtain the Web application that supports the BPs specified in the BPM. Specifically, this application is generated for the Tapestry Web Framework. For this reason, according to the architecture imposed by this framework, these transformations produce a set of .java, .page and .html files.

Although model transformation is a relatively young area, there already exist different possibilities to perform this task (Czarnecki $\&$ Helsen, 2003). Specifically, the options that have been taken to implement the transformations defined in this work are the Atlas Transformation Language (ATL ${ }^{29)}$ to implement M2M transformations and MOFScript ${ }^{30}$ to implement M2T transformations. A key factor on the selection of the

\footnotetext{
29 http://www.eclipse.org/m2m/atl/

$30 \mathrm{http}: / /$ www.eclipse.org/gmt/mofscript/
} 
transformation languages was their tool support in order to apply the proposal in practice. The usage of Eclipse-based tools could permit seamless tool integration with other projects used by the present work such as Eclipse Modelling Project ${ }^{31}$ and SOA Tools Platform Project ${ }^{32}$.

The ATL language (Jouault \& Kurtev, 2006) is a proposal developed in parallel to the OMG QVT (OMG, 2005a) standard for model transformation (Miller \& Mukerji, 2003). ATL is a hybrid language (declarative and imperative) that allows the definition of rules that describe how source model elements are matched and navigated to create and initialize the elements of the target models. Moreover, it provides an IDE developed on top of the Eclipse platform, which provides a number of standard development tools providing support to the edition, execution and debugging of M2M transformations.

With regard to M2T transformations, MOFScript was one of the candidates in the OMG RFP process on MOF Model to Text Transformation. It is included in an Eclipse subproject aimed at developing tools and frameworks for supporting model to text transformations, e.g., to support generation of implementation code or documentation from models. Some of the characteristics that made this tool a good candidate to deal with M2T transformations were that (1) it allows generating text from MOF-

\footnotetext{
31 http://www.eclipse.org/modeling/

32 http://www.eclipse.org/stp/
} 
based models, (2) it defines a set of mechanisms that facilitates the implementation of transformations (i.e., control mechanisms, string manipulation, expressions to reference model elements), (3) it allows specifying the file being generated and (4) it allows keep traceability between source models and generated files.

\subsection{Model Transformations to Generate WS-BPEL code}

The main goal of the BPMN notation is bridging the gap between the business process design and the process implementation. The way to bridge this gap has already been documented (White, 2005) and even implemented in some tools (Borland Together ${ }^{33}$ or Oracle BPEL Manager ${ }^{34}$ ). With regard to the published documentation, this is based on a particular example and does not provide a complete mapping between BPMN diagrams and WS-BPEL. On the other hand, the limitations of WS-BPEL forces extending the language in proprietary ways, which works against the language promised portability. If the required extension were based on Web services interfaces, the portability would not be compromised. However, most vendors add proprietary extensions to lock users in their products.

Therefore, we needed to provide a transformation that (1) would cover the whole mapping between the graphical notation and the

\footnotetext{
${ }^{33} \mathrm{http}: / /$ www.borland.com/us/products/together/index.html

${ }^{34} \mathrm{http}: / /$ www.oracle.com/technology/bpel/index.html
} 
executable language and (2) would generate an executable representation $100 \%$ compliant with the standard. In this direction, we found within the Babel project ${ }^{35}$ developed by the Business Process Management Group ${ }^{36}$ the BPMN2BPEL ${ }^{37}$ tool. This tool is a Java application that transforms process models represented in BPMN diagrams into process definitions represented in WS-BPEL (Ouyang et al., 2006). This tool seems initially promising but it has some limitations:

1. It does not include a graphical editor for BPMN modelling. Although providing graphical editors to build models is not a must for the realization of the MDD approach, it constitutes in most cases the key factor for the success of any tool.

2. The generated WS-BPEL code is incomplete and only generates the code related to activities within a WS-BPEL document. The completeness of a WS-BPEL document is necessary in order deploy successfully the process definition into a process engine.

As can be seen in Figure 9.1, the WS-BPEL complete code generation process has been defined using both M2M and M2T transformations and includes the following steps:

\footnotetext{
35 http://www.bpm.fit.qut.edu.au/projects/babel/

${ }^{36} \mathrm{http}: / / \mathrm{www} . b p m . f i t . q u t . e d u . a u /$

37 http://www.bpm.fit.qut.edu.au/projects/babel/tools/
} 
1. First of all, BP definitions depicted graphically following the BPMN notation are transformed into BPs according the Babel tool format.

2. In parallel to the first step we can generate the interface (WSDL file) of the WS-BPEL process as well as the data types used by it. The interface is generated in the WSDL language since the executable WS-BPEL process is seen from the outside as a Web service.

3. Once we have a business process representation according to the schema defined by the Babel tool we can execute the BPMN2BPEL tool to obtain a preliminary version of the WS-BPEL document.

4. Finally, the previously obtained WS-BPEL document is completed by including the sections that are not built by the Babel tool (which are the partner link, variables and correlation set sections) and by associating activities with the proper partner. Moreover, before sending and receiving data to and from an external partner it is necessary to perform a transformation process to map the data types used by the process to the ones used by the external partners. Therefore, it is necessary (1) to build a new partner in charge of this transformation process and (2) to include the activities that invoke the operations providing this functionality.

After the application of this set of steps we obtain a complete executable WS-BPEL document which can be executed in a WS- 
BPEL engine. The following subsections present in detail each of these steps.

\subsubsection{From the BPM to BPMN according to the Babel Tool}

In the context of M2M transformations, we have defined and implemented the necessary transformation rules to overcome the BPMN modelling limitation of the BPMN2BPEL tool. Since the metamodel used by the Babel tool is quite simple (considering just tasks, links and gateways to control the flow) the set of transformations defined in this case are quite straight forward as the following list shows:

- Activities are transformed into nodes (see Code excerpt 9.1)

- Links, which connect two nodes, are transformed into arcs (see Code excerpt 9.2)

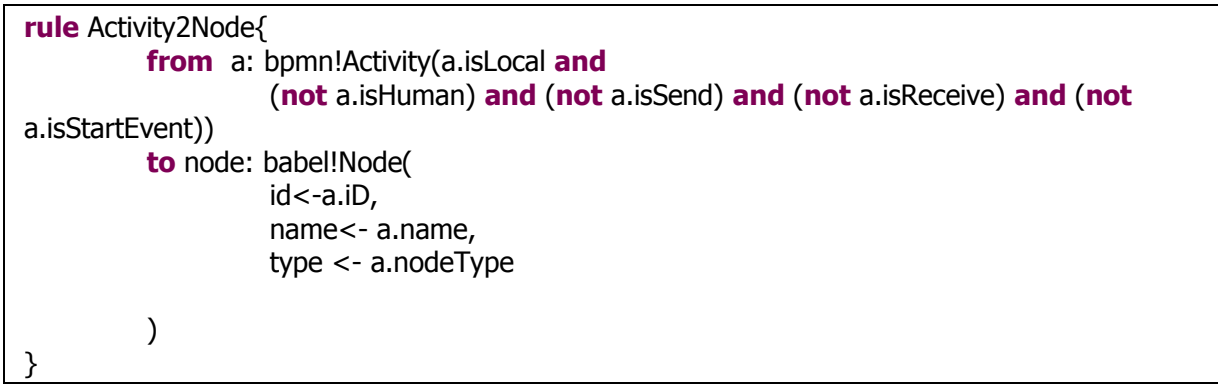

Code excerpt 9.1 Rule transforming Activities into nodes

rule Sequence2Arc \{

from s: bpmn!SequenceEdge(s.isLocal and not s.target.isInitial)

to arc: babel!Arc(

id<-s.iD,

source $<-$ if s.source.isHuman and (not s.source.isInitial) then

thisModule.Human2Complete(s.source).id

else 


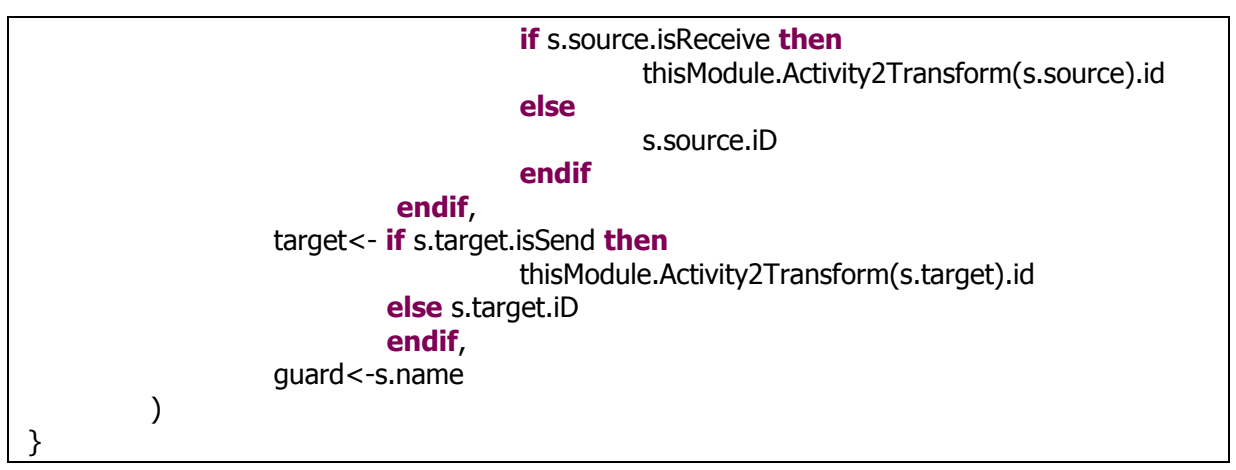

Code excerpt 9.2 Rule transforming Links into arcs

In addition to these two transformation rules, a set of additional helpers functions have been defined in order to inquire some properties about elements of the model. This is necessary since BPMN considers more activity types than the ones supported by the Babel format. Therefore, these helpers constitute the mappings between the BPMN and the Babel activity types. In the Code excerpt 9.3 the helper defining the mappings between the whole set of BPMN start events and the one supported by Babel is presented.

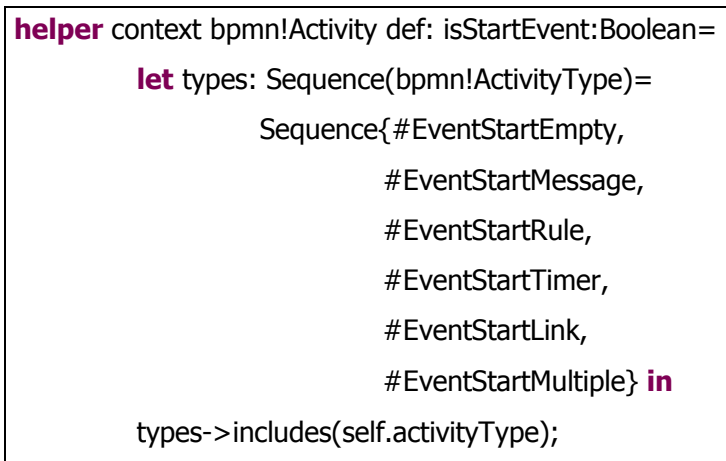

Code excerpt 9.3 Helper to check whether an activity is defined as a start activity 
A set of helpers have been defined to determine the type of the activity being handled (isStartEvent, isMessageEvent, isTimerEvent, isXorJoin, isXorSplit, isEbXorJoin, isEbXorSplit, isAndJoin, isAndSplit, isEndEvent, isTask, nodeType). For the complete definition of these helpers see Appendix $\mathrm{D}^{38}$.

However, as we have already mentioned, the WS-BPEL document generated by the Babel tool constitutes a preliminary version of the document. The code presented in the Code excerpt 9.4 shows the WS-BPEL document generated for the "Request Book Purchase" process (see Appendix C) by the Babel tool.

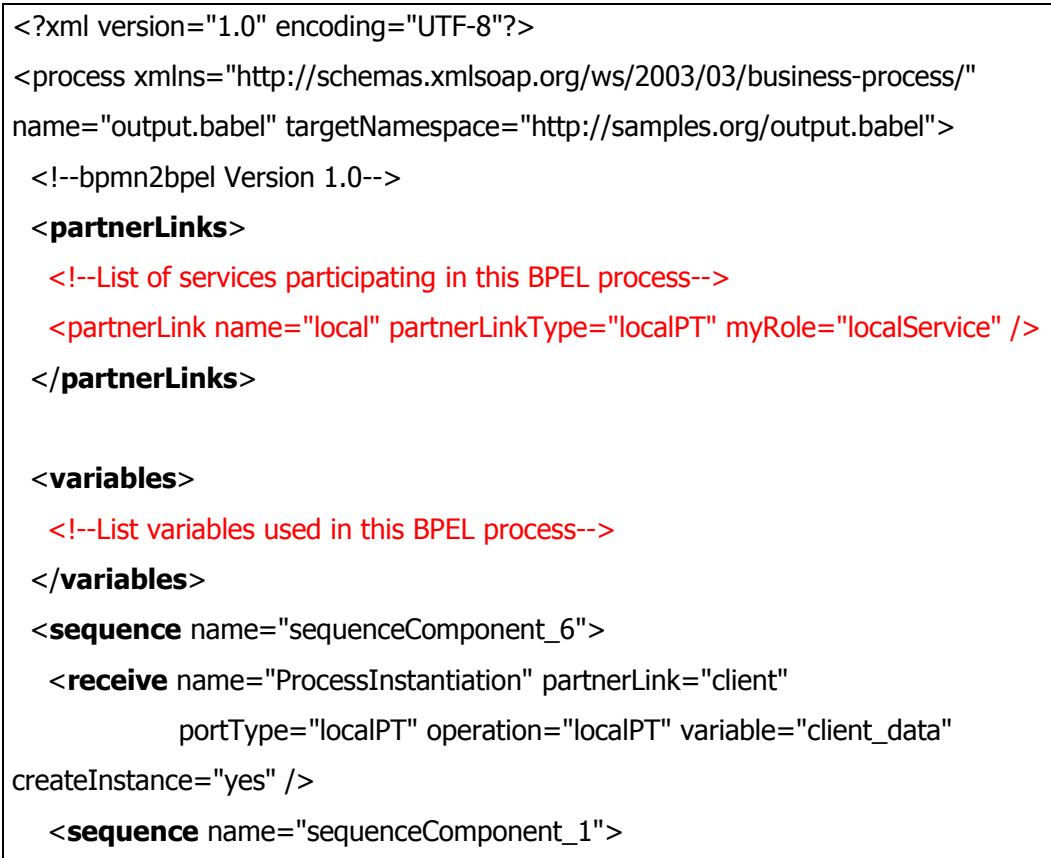




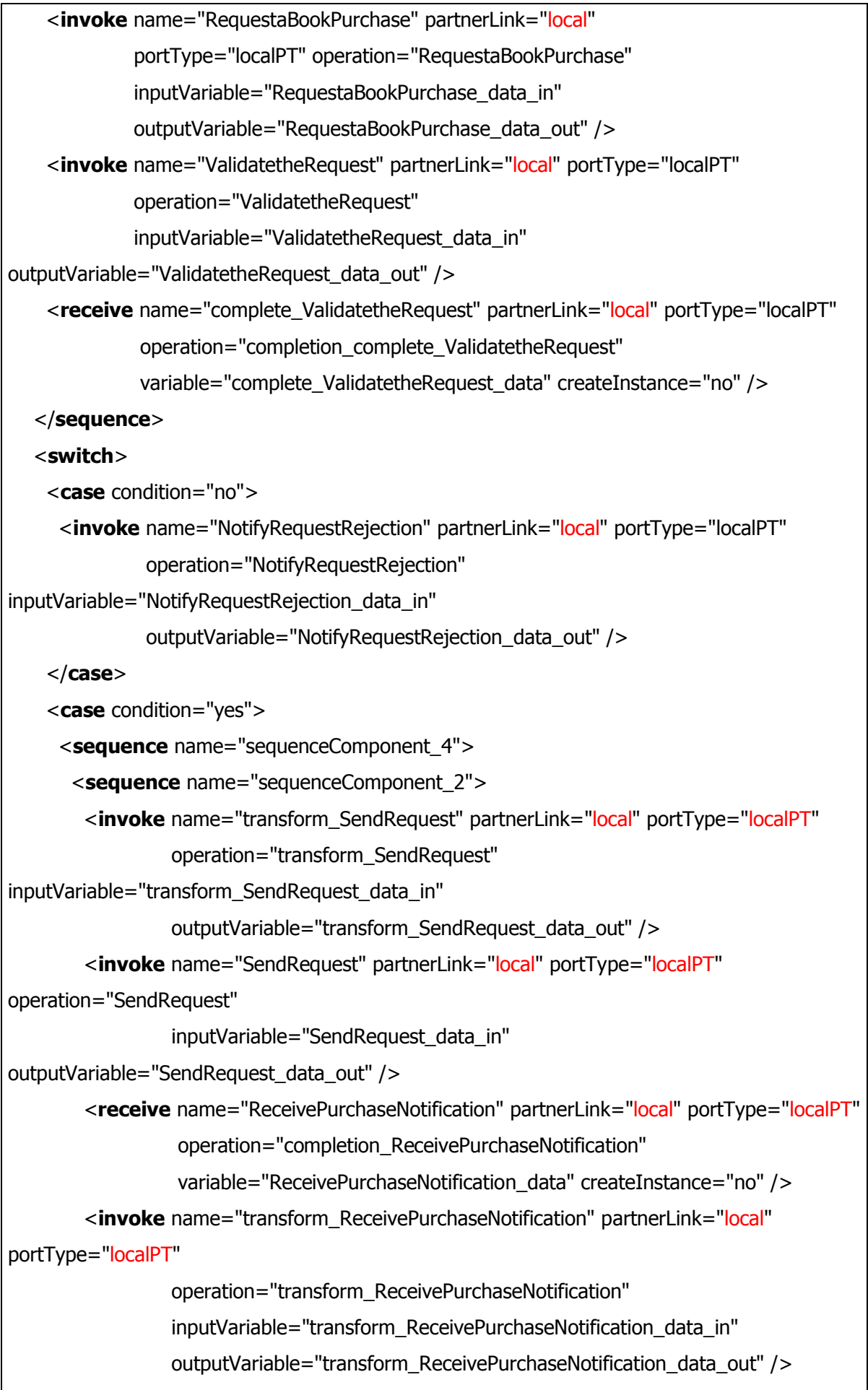




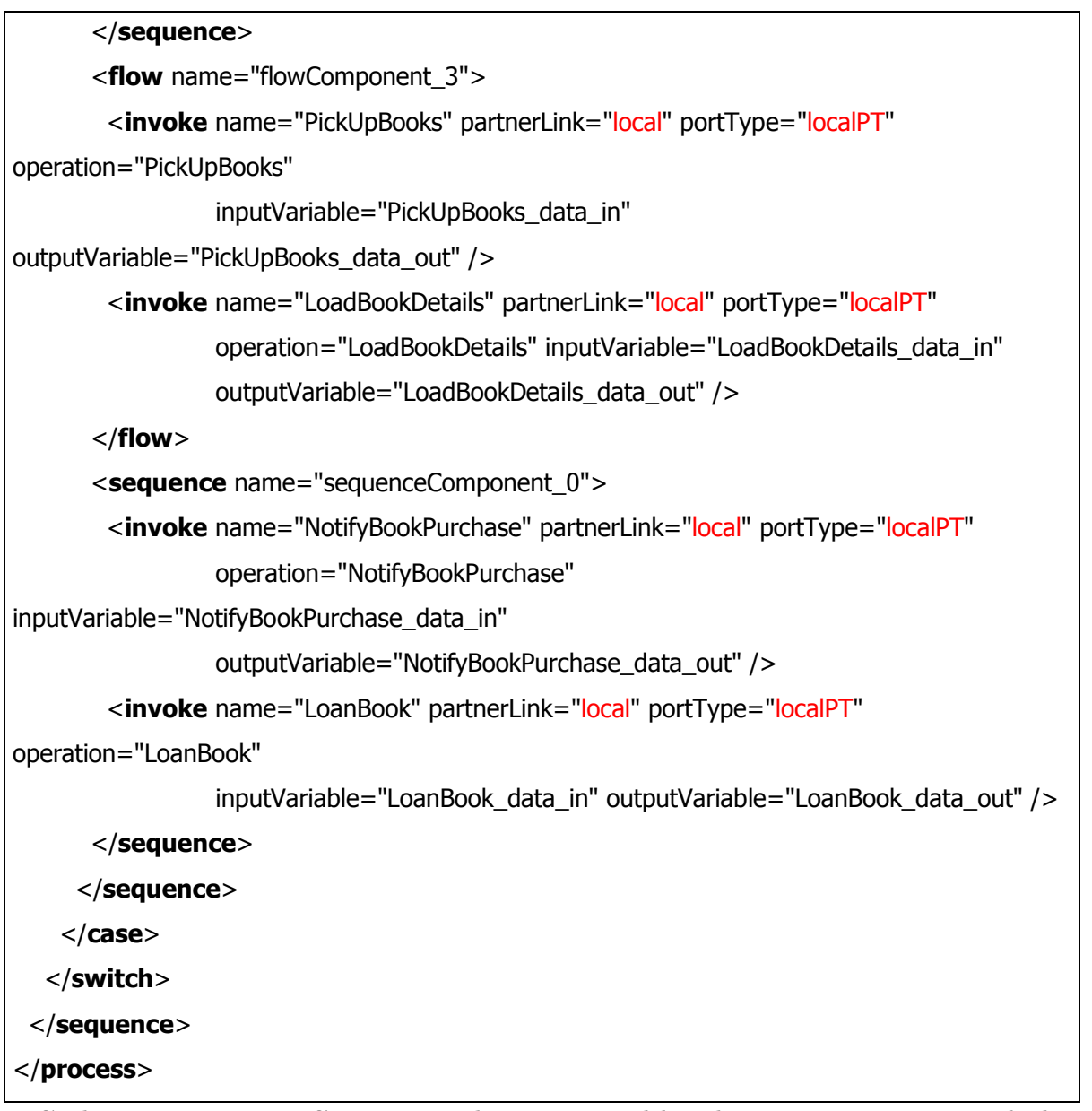

Code excerpt 9.4 WS-BPEL code generated by the BPMN2BPEL Babel tool

As the Code excerpt 9.4 shows, in order to generate a valid WSBPEL document the Babel tool generates:

1. the partner link section, but just including one partner link which is associated to all the activities of the process

2. the variables section, where only the structure is defined, no variables are defined in it. 
Finally, regarding the Correlation Set section, not even the structure is built. This limitation of the Babel tool is due to the lack of expressivity of the metamodel handled by it. In fact, this metamodel is only composed of two primitives which are nodes and $\operatorname{arcs}$ (the whole metamodel is shown in Appendix $\mathrm{D}^{39}$ ). Therefore, it is necessary to add the information that cannot be generated by the Babel tool.

\subsubsection{Completing the WS-BPEL Document}

The following subsections explain the transformations defined to complete the WS-BPEL document generated by the Babel tool. These explanations are accompanied with some interesting code excerpts which are also presented in appendixes ${ }^{40} \mathrm{D}, \mathrm{E}$ and $\mathrm{F}$.

\section{Adding the Partner Link and Namespace sections}

The first section that we are going to focus on is the section related to the partners interacting with the process. Before going deep into the details, Figure 9.3 depicts graphically the interaction schema that is going to be used for each generated WS-BPEL document.

http://www.dsic.upv.es/ vtorres/thesis/appendixes/appendixD

${ }^{40}$ Available on-line at http://www.dsic.upv.es/ vtorres/thesis/appendixes 


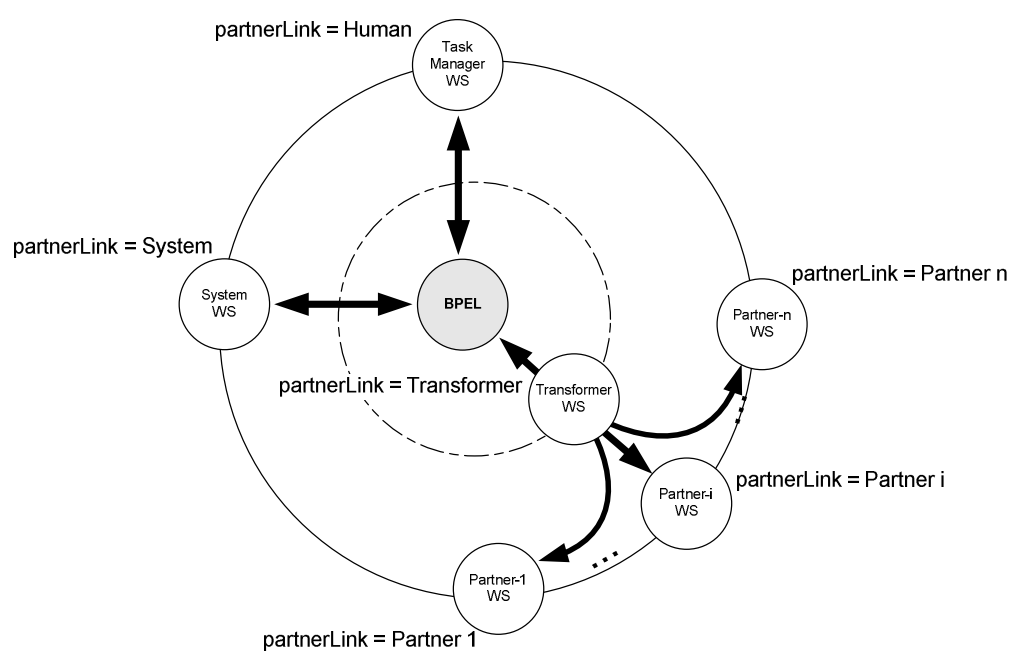

Figure 9.3 Interaction between the WS-BPEL process and the set of Partners

As Figure 9.3 shows the WS-BPEL process interacts with four different types of Web services which are the Task Manager WS, the System WS, the Transformer WS and the collection of Partner$i W S$.

1. The Task Manager Web service has been defined to overcome the WS-BPEL lack for handling human tasks. If the WS-BPEL process includes tasks involving human participation, this service must be always present as a partner. This service provides a set of operations that are invoked when a task demanding human participation is reached by the process flow (see chapter 8 for a detailed description of this service).

2. The System Web service is the partner in charge of providing all the functionality defined in the local system and that is associated with process activities. Therefore, 
this service will only be generated when the WS-BPEL process invokes functionality defined in the local system.

3. The Transformer Web service is a service in charge of performing format conversions between the data types used by the WS-BPEL service and the data types used by each of the business partners. This service is, by the moment, built manually. However, based on works such as (Benatallah et al., 2005) where adapters are defined automatically to integrated different Web services, this service would be built automatically.

4. Finally, the set of Partner-i Web services represent each one of the external business partners involved in the process. There is one per each business partner.

The Code excerpt 9.5 shows the ATL code defined for the construction of the four types of partners interacting with the WSBPEL process. This code can be found in Appendix D.

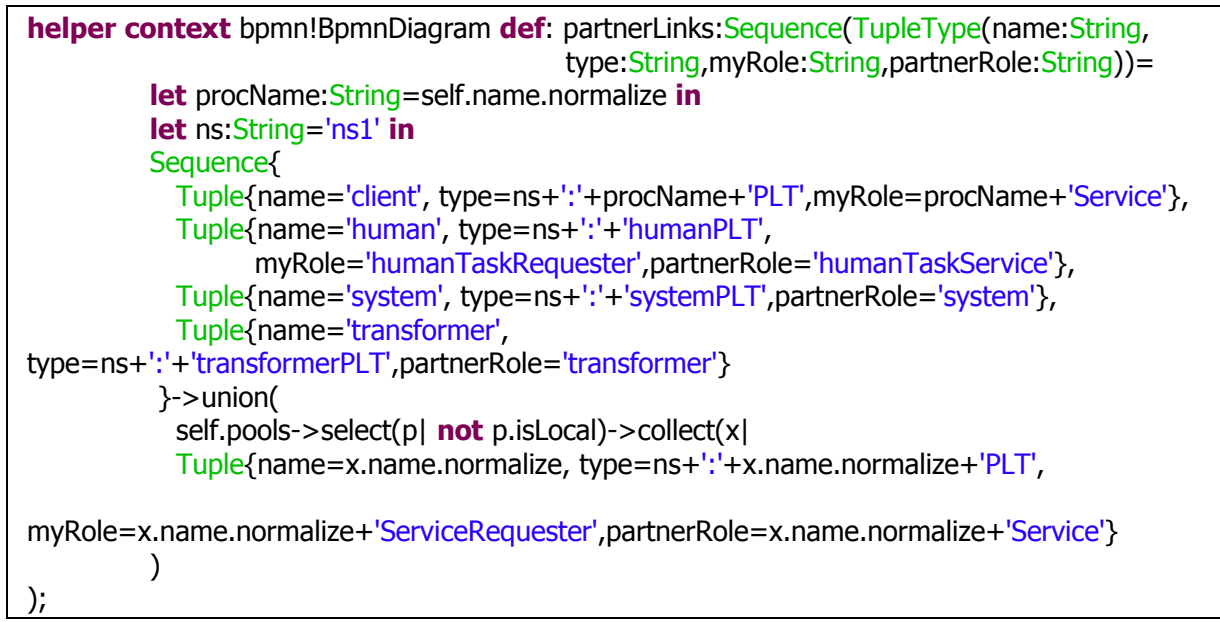

Code excerpt 9.5 Helper building the Partner Link section 
Moreover, it is also necessary to include the namespace section to the WS-BPEL document. The code excerpt in charge of adding this section is shown in Code excerpt 9.6.

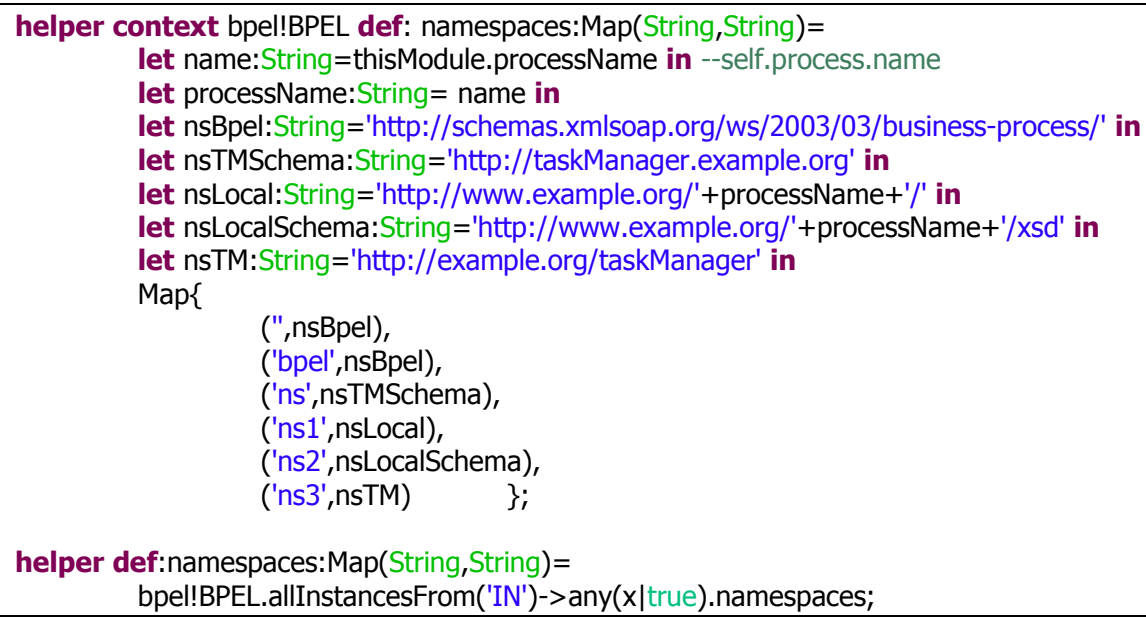

helper def:namespaces:Map(String,String)= bpel!BPEL.allInstancesFrom('IN')->any(x|true).namespaces;

Code excerpt 9.6 Helper related to the generation of the Namespace section

In these two cases we have included the namespaces explicitly in the code. However, in a next version of the tool we want to allow the parameterization of this part of the transformations.

\section{Adding the Variables section}

By default, the Babel tool associates input and output variables to all the invoke activities defined in the WS-BPEL document. The nomenclature used is the activity name followed by “_data_in” or “_data_out” depending on the case (i.e ValidatetheRequest_data_in and ValidatetheRequest_data_out). However, the process still needs the inclusion of a set of variables that will be associated to activities that are going to be generated later on. The set of new variables are defined to: 
- Be included in the reply activities added as a response to the receive activities (i.e. client_dataResponse)

- Gather the output values of the Invoke activities when the Transformer Web service is invoked (i.e. SendRequest_data_mapped or ReceivePurchaseNotification_mapped)

- Gather the input and output values send and received from the invocation of the "addTask" operation provided by the Task Manager Web service (i.e. addTaskRequest)

- Represent the object created and modified along the process instance (i.e. BookRequest)

The Code excerpt 9.7 shows how variables are built depending on their purpose and the place where they are used. For instance, associated to a send activity (activity that invokes an external partner) two variables are defined, one to gather the input data for the activity and another to gather the input data contained in the previous variable but this time with the types according to those used by the external partner.

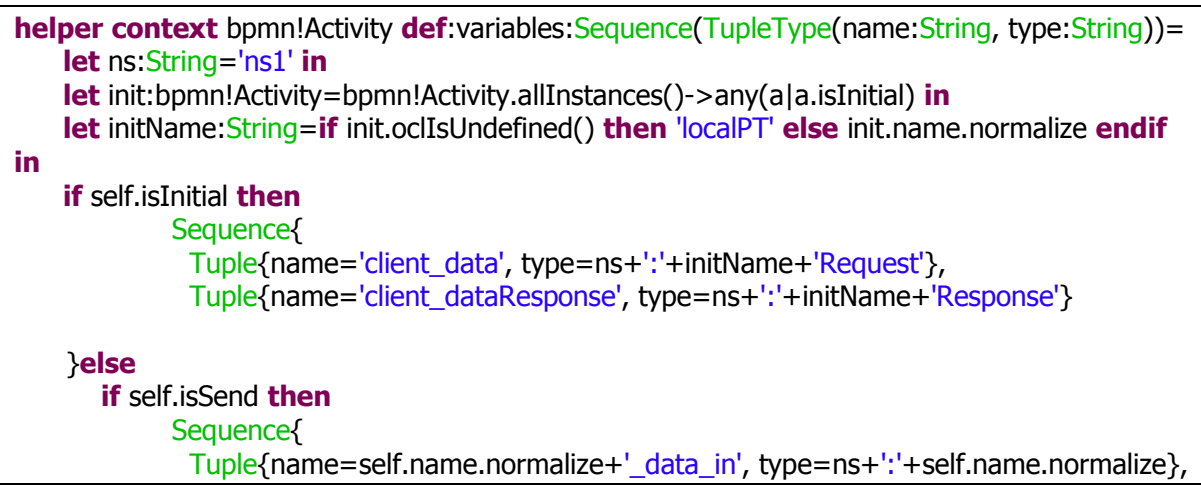




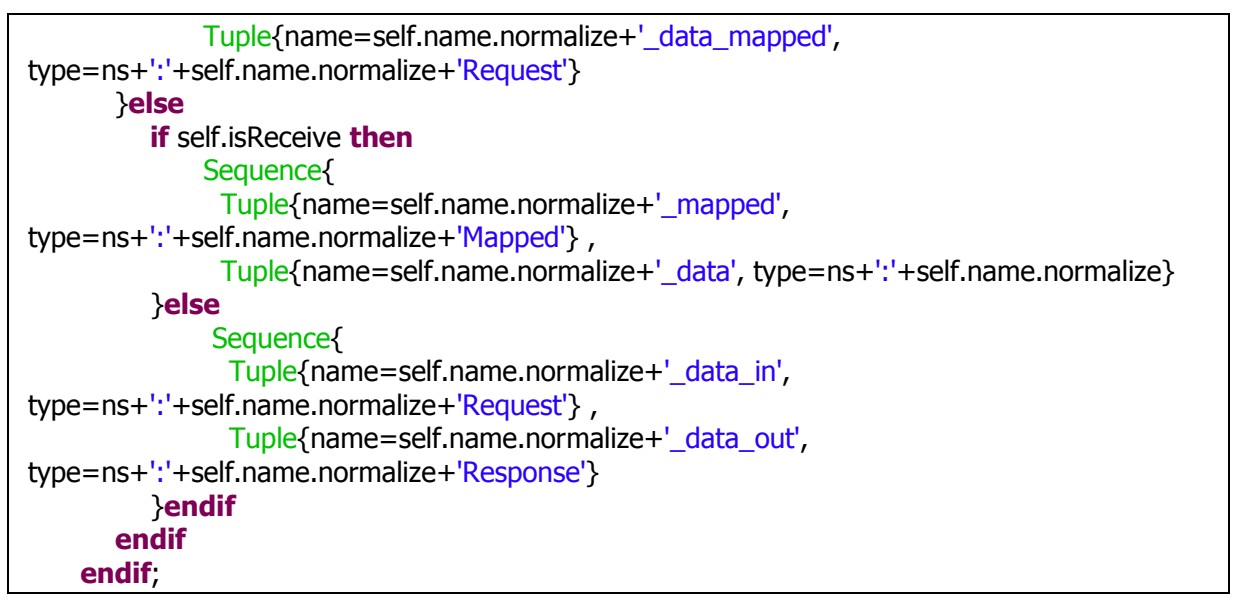

Code excerpt 9.7 Helper building the required Variables

\section{Adding the Correlation Set Section}

Messages received by BPs require some data in order to identify the process instance in which they are involved. This ensures that messages are dispatched to the correct process instance. Therefore, in order to define a value that is going to identify each process instance, we create the correlation set section. In particular we are always going to define a correlation set named "CS1" whose value is going to be given by the identifier of the object created and manipulated along the process instance life. For this purpose, the code shown in the Code excerpt 9.8 creates this section.

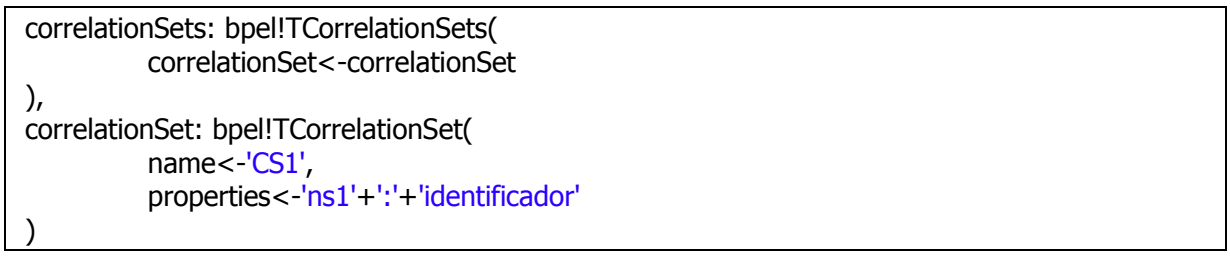

Code excerpt 9.8 Code Excerpt creating the Correlation Section 
Moreover, as correlation set names are used in invoke, receive and reply activities, within the transformations that handle this type of activities we are going to associate them the generated correlation set. An example of the code included after these kinds of activities is shown in Code excerpt 9.9.

<bpel:correlations>

$<$ bpel:correlation initiate="no" set="CS1"/>

$</$ bpel:correlations $>$

Code excerpt 9.9 Correlation section associated to invoke, receive or reply activities

The initiate attribute is set to "no" or "yes" depending if the receive activity corresponds to the first activity of the process which creates an instance of the process.

\section{Recovering the Business Process Extension Information}

We defined an extension to the original BPMN notation to allow transforming automatically BPMN diagrams into WS-BPEL code (see chapter 5 for the details). However, since the Babel tool lacks traceability support for the original elements of the BPMN notation we have to recover all the information that we loosed after the execution of this transformation. With this data we can complete the WS-BPEL code generated by the Babel tool by specifying Assign activities and Receive and Invoke attributes such as partner links and port types. 
At this point the code that is still missing in the new definition of the WS-BPEL document refers to (1) assign activities and (2) some attributes (partnerLink and portType attributes) of process activities. Table 9-1 summarizes the actions performed by the defined transformations depending on the kind of activity. The Activity type column refers to characteristics that differentiate BPMN activities:

- User to denote activities performed by the system but requiring human participation.

- Manual to denote activities that are completely performed by humans.

- Initial to denote the first activity defined in the process.

- External partner to denote activities used to communicate with external services.

- Transformer to denote the activities used to communicate with the transformer service (service in charge of mapping the data types handled by the process with the data types used by the external partners).

\begin{tabular}{|c|c|c|c|c|c|}
\hline & Activity Type & PartnerLink & portType & $\begin{array}{c}\text { Assign } \\
\text { activity? }\end{array}$ & $\begin{array}{c}\text { Create } \\
\text { instance? }\end{array}$ \\
\hline \multirow{5}{*}{ 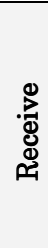 } & User & Human & completeHumanTask & yes & No \\
\hline & Manual & Human & completeHumanTask & & No \\
\hline & Initial & - & - & Yes & Yes \\
\hline & Ext. partner & Partner-i & Completion_<partner_name $>$ & & \\
\hline & Transformer & Transformer & transformPT & Yes & No \\
\hline \multirow{4}{*}{ 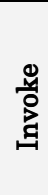 } & User/Manual & Human & taskManagerPortType & Yes & No \\
\hline & Service & System & doTask & Yes & No \\
\hline & Ext. partner & Partner-i & $<$ partner_name $>$ PT & & No \\
\hline & Transformer & Transformer & transformPT & Yes & No \\
\hline
\end{tabular}

Table 9-1 Actions performed during ATL activity transformations 
Regarding the values indicated in the remainder columns:

- The Human partner link refers to the service that mediates between the process and the user.

- The completeHumanTask port type refers to the operation from the Human service in charge of completing a user task

- Assign activities are required in all cases since it is necessary to prepare the data to be sent and received to and from partners.

- The createInstance attribute is only set to "yes" only for Receive-Initial activities which means that an instance of the business process is going to be created if it does not already exists.

- The <partner_name> used in the names of the port types for Invoke and Receive activities when these are associated to an external partner refers to the service provided by the partner.

- For Invoke-User/Manual activities the Assign section has also to prepare: (1) the group to which the target user belongs, (2) the name of the task, (3) the user involved in the task and (4) the current data. All this data is required since the process is going to invoke an operation of the Task Manager WS.

Finally, we want to note that after the execution of these transformations we have a WS-BPEL "model" representing the WS-BPEL code. However, since we have created the EMF metamodel of WS-BPEL from the corresponding XML Schema 
document, the serialization of the model to the code is automatically performed by EMF.

\subsubsection{XSD \& WSDL Generation}

In addition, to complete the WS-BPEL generated code, it is necessary to make this WS-BPEL code deployable. For this reason it is necessary to generate also the interface associated to the new service defined by the WS-BPEL document (expressed in WSDL) and the data types used by it (expressed in XSD). In this case, also the Structural and Services model are used to produce these two files. Specifically, these two models contain a detailed description of the operations and data types used and produced by the functionality associated to BP activities. Code excerpt 9.10 shows how these models are specified at the header of the M2T transformation which corresponds to:

1. the BPMN metamodel

2. the extension defined to the BPMN notation (defined in the bpmnx metamodel)

3. the OOWS metamodel, which includes the Structural, Services, Navigational and Presentation metamodels.

texttransformation ExampleTransformation (in bpmn:"http://stp.eclipse.org/bpmn", in ext:"http://www.example.org/bpmnx", in oows:"http:///oows.ecore") \{

Code excerpt 9.10 Header of the MOFScript transformation

In Code excerpt 9.11 the entry point of the transformation is defined. In it a set of functions are invoked which are in charge of the generation of each section of the WSDL document (header, import, types, messages, portTypes, bindings, services, 
partnerLinks and property alias). The complete set of transformations is included in Appendix $\mathrm{E}^{41}$.

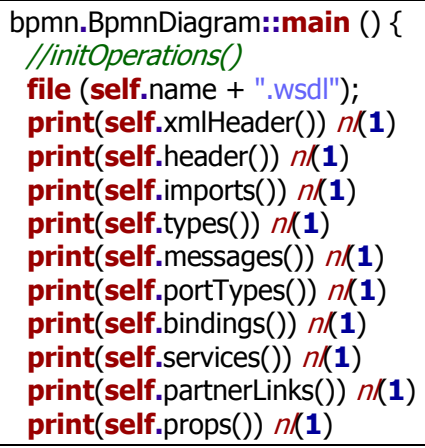

Code excerpt 9.11 Entry point rule to the transformation to obtain the

WSDL file

The properties and property aliases included in the WSDL file are used to define the correlation set (set of properties shared by different messages) defined in the WS-BPEL process. On the one hand properties define the identifiers used as correlation set in the WS-BPEL document. On the other hand, since a single property might exist in several messages, property aliases allow extracting the value of the property from a message or variable.

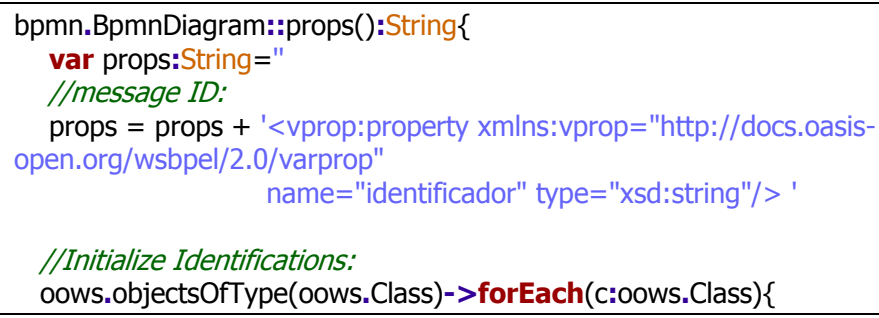

41

Available

on-line at

http://www.dsic.upv.es/ vtorres/thesis/appendixes/appendixE 


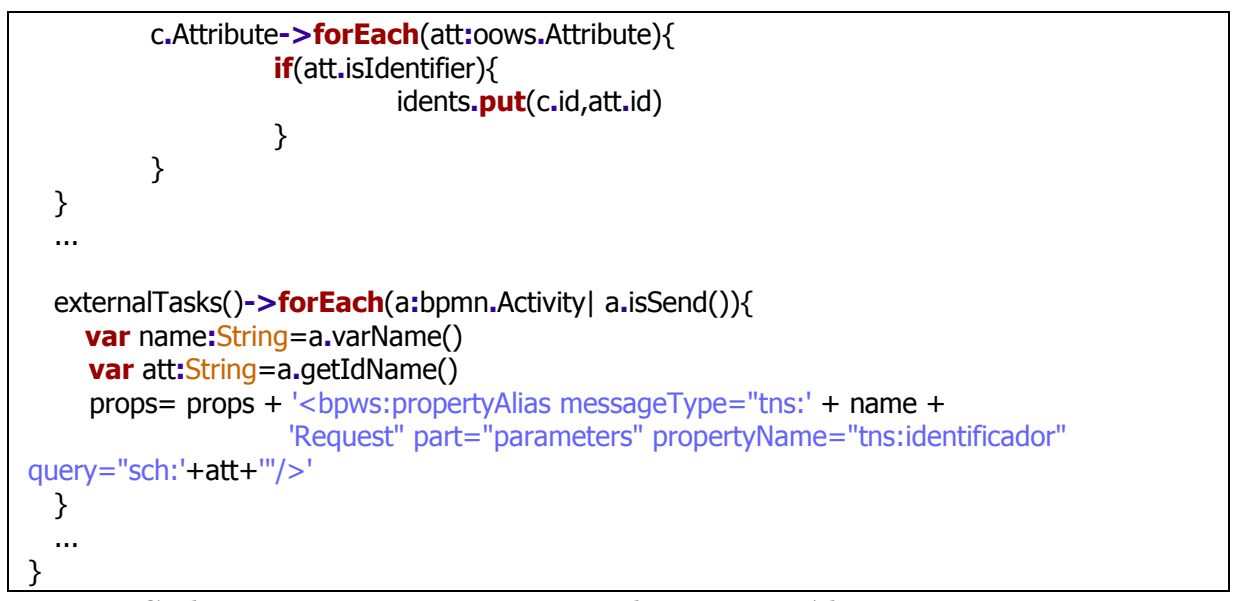

Code excerpt 9.12 Property and Property Aliases generation

Regarding the generation of the schema document (XSD file), a MOFScript transformation has been defined. In Code excerpt 9.13 the entry point of the transformation is defined. In it a set of functions are invoked which are in charge of the generation of each section of the XSD document (header, types and elements). The complete set of transformations is included in Appendix ${ }^{42} \mathrm{E}$.

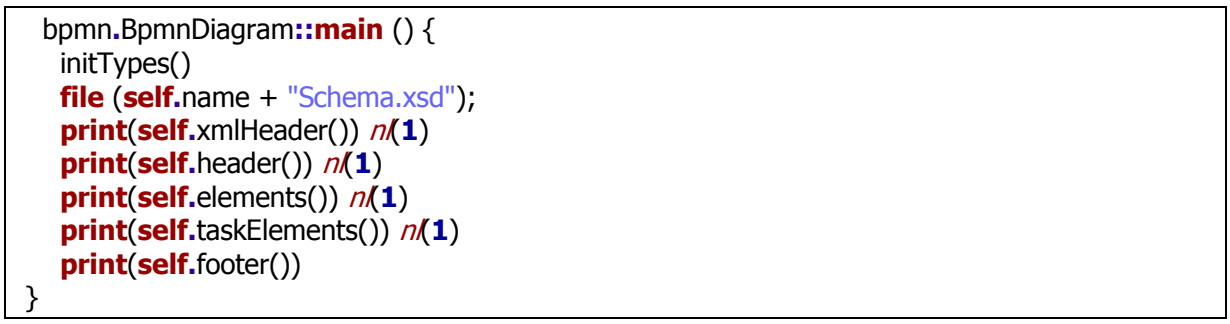

Code excerpt 9.13 Entry point rule to the transformation to obtain the XSD file 
In this case we generate separately data types and elements. The operations in charge of this generation are elements() and taskElements() respectively.

\subsection{Model Transformations to Generate the User Interface}

The second type of artefact obtained from the models built at the modelling level corresponds to the different code files that constitute the Web application. The different transformations (M2M and M2T) that have been defined in this case were briefly presented Figure 9.2 and produce the following assets:

- The Navigational Model that supports the execution of the original BP definitions.

- The set of files corresponding to the Navigational Model in terms of the Tapestry Web Framework.

The following subsections detail the process followed in each case.

\subsubsection{BPMN to OOWS Navigational Model}

The set of M2M transformations defined to obtain a first version of the OOWS Navigational model uses as input the BPM model as well as the Structural and Services models. The particularity of these transformations is that only apply to tasks that are included in lanes defined in the BPM as "role-one» and "role-any» which correspond to tasks performed by human beings. In the following paragraphs we present textually the set of rule transformations defined to obtain the OOWS Navigational model. The 
Navigational model built after the application of these transformations includes the extension defined in this thesis (see chapter 6). The complete set of rules expressed in ATL is presented in Appendix G.

\section{Transformations Rules:}

- Process Context Generation. For each lane defined in the process with its typed valued as «role-one» or "role-any» we build a Process Context with the same name in the Navigational Map. We want to note that the roles used in the $\mathrm{BP}$ definition correspond to one of the type of users defined in the User Model. (see Code excerpt 9.13).

- Activity Container Generation. Each task involving human participation («user» and "manual» tasks) defined within the boundaries of a role type Lane («role-any» or "role-one») will be transformed into an Activity Container with the same name of the task (see Code excerpt 9.13).

- AIU Generation: Depending on the type of the task, a different type of AIU will be generated:

- For the tasks that are defined as "user», we include a Main-AIU within the corresponding Activity Container.

- If the operation associated to the task refers to an operation defined in the Structural Model, then include a Class-View in the Main-AIU that references the class containing such operation. 
- If the operation defined in the task refers to an operation provided by a Service from the Services Model, then include a ServiceFunctional-View in the Main-AIU that references such operation.

- For the tasks defined as "manual», we include a Human-AIU within the Activity Container.

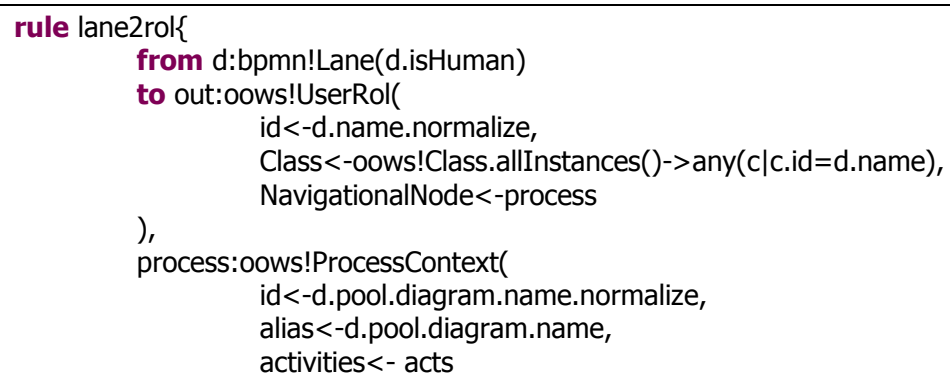

Code excerpt 9.14 ATL rule to build a Process Context

Code excerpt 9.13 shows the ATL rule that implements the transformation that builds a Process Context and the structure of the set of Activity Containers included in it (Appendix G contains the complete set of transformations).

After the application of these transformations to the BPM, we obtain a basic Navigational Model that will allow users executing 
the corresponding BPs. Based on the case study developed in Appendix C, Figure 9.4 depicts the Process Context generated for the secretary role.

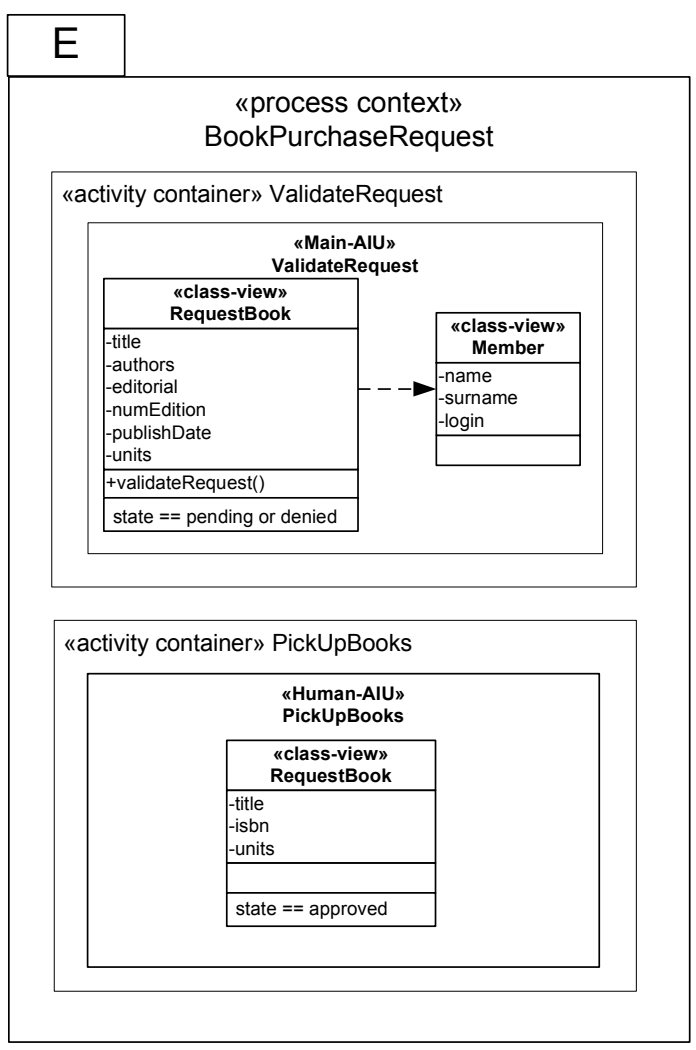

Figure 9.4 Basic Process Context Generated for the Secretary role

This Process Context includes two activity containers (one for each user task assigned to the secretary role), among which are a MainAIU and a Human-AIU. 


\subsubsection{Code Generation for a Web Framework}

Navigational and Presentation models defined by OOWS include web page abstractions that can be mapped onto any language or framework capable of implementing web applications. In particular, in this work we decided to make use of a web framework that facilitates the construction of the final application. From the wide range of available web frameworks we finally chose the open-source framework Tapestry ${ }^{43}$. Briefly, the main reasons for this decision were the use made by Tapestry of (1) a component-based model, (2) the Inversion of Control (IoC) pattern and (3) the Model-View-Controler (MVC) pattern.

A Tapestry component, technically know as JWC (Java Web Component) is a web component that can be configured, instantiated and aggregated to take part in other components. These components can be from an input text to a flow control component such as an "if" clause. In addition, the set of components is extensible allowing the developer defining her own components.

The $I o C$ pattern avoids the software developer dealing with low level tasks such as controlling references between objects. This task is relegated to a container (which is called HiveMind ${ }^{44}$ in the

\footnotetext{
43 http://tapestry.apache.org/

${ }^{44} \mathrm{http}: / /$ hivemind.apache.org/
} 
Tapestry framework) which injects all dependencies, references and objects related to a given object.

The $M V C$ pattern is a software architecture pattern that separates in three different components the data, the presentation and the logic of the application. This pattern is widely used in the development of web applications, where the View is constituted by HTML templates and the code providing dynamic data for the page, the Model is constituted by the data model (which is usually represented by a class diagram) and the Controller is constituted by the logic of the application.

As a result of this decision, the obtained benefits are the following:

- Simplicity, consistency and efficiency of the built code.

- Tapestry uses standard HTML code for their templates. Moreover, following the IoC pattern, the semantics of the components is included in separate configuration files, fact that allows keeping templates just with HTML code. Moreover, we can reuse HTML code by converting it into a Tapestry component, which can then be used by means of the proper identification.

- Tapestry components are easy to use but also to create.

- The use of the IoC pattern avoids the developer dealing with low level aspects.

- It includes useful predefined components such as an input validation system, an error report, own AJAX components, etc. 
From the required code to obtain a complete web application, we group them into two groups, which are (1) generic code independent of the domain and (2) specific code dependent of the domain. The code belonging to the first group has been already built and is going to be reused in each new developed system. For example, the files required to start the application (such as the home.html, home.page and home.java) are considered within this group. On the other hand, the code belonging to the second group is going to be generated each time based on the models that define the system. Figure 9.5 outlines the stack of abstraction levels of the generated application. In addition remarks the fact that only the application dependent code is generated from the models defined in the method.

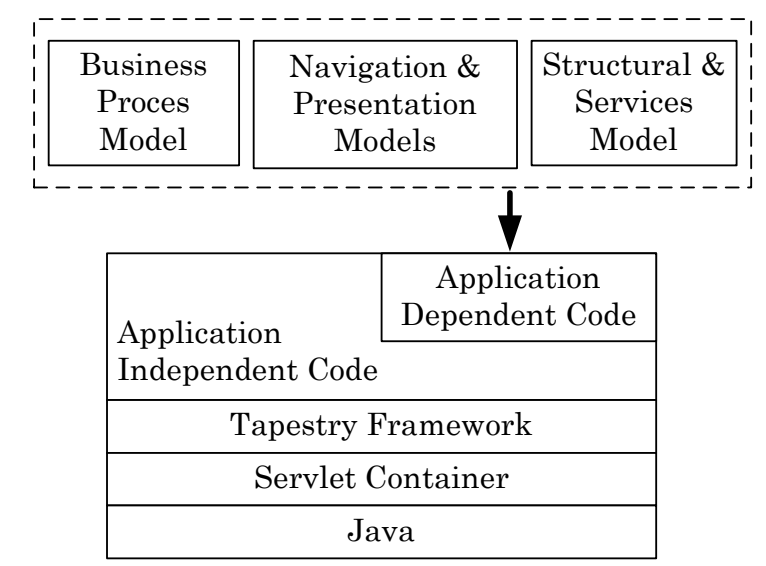

Figure 9.5 Web Application Abstraction Levels

The transformations implemented for the generation of the application for the Tapestry framework have been organized in six different files. These are explained in the following paragraphs. 
Transformations associated to the process itself:

- Model.m2t: This transformation generates the java class (Model.java) in charge of keeping the information about the existing processes, activities and roles. Moreover, it also keeps its data and the way in which processes, activities and roles are related.

- ProcessJava.m2t: This transformation generates the java class (Processes.java) in charge of redirecting the user to the appropriate web page depending on the state of the process.

Transformations associated to process Activities:

- ActivitiesHTML.m2t: It generates the HTML code of the application (the view from the MVC pattern).

- ActivitiesPage.m2t: This transformation generates the .page files gathering the XML code corresponding to the declarations of the Tapestry components.

- ActivititesJava.m2t: It generates the java code associated to the HTML generated by the ActivitiesHTML transformation (the controller from the MVC pattern).

Transformations associated to the Structure of the Domain

- ClassDiagram.m2t: It generates the classes defining the domain of the application (the Model from the MVC pattern). Since the logic of the local system is going to be provided by the OlivaNova tool, the generated classes in this case represent locally the objects provided by the tool. This integration is possible since applications generated 
with the OlivaNova Transformation Engines provide Components and Web Services to make use of the application logic. In both cases, domain classes are available to be queried and to access their business logic functionality.

For a whole description of these transformation see Appendix $\mathrm{H}^{45}$, where all the MOFScript code to implement these transformations is included.

After the application of these transformations, a ready to run Web application is built. The different generated files are distributed among different folders according to the Tapestry requirements. A complete description of this structure is presented in chapter 10 .

\subsection{Conclusions}

This chapter has presented in detail the set of transformations that have been defined to move the system specification performed following the OOWS approach to a particular implementation. As transformation languages we have used ATL and MOFScript to implement M2M and M2T transformations respectively. On the one hand we have detailed the process performed to obtain from a business process specification depicted in the BPMN "extended" notation to an equivalent definition of the process in the executable WS-BPEL language. For this purpose, in addition to
45
Available
on-line at

http://www.dsic.upv.es/ vtorres/thesis/appendixes/appendixH 
the already mentioned transformation languages we have made use of the BPMN2BPEL java tool. This tool allows us generating a preliminary version of the executable process in the WS-BPEL language. Then making use of the BPM, Structural and Services models we have completed the WS-BPEL document and also generated the file defining the interface of the process (in a WSDL file) as well as the data types used by it.

On the other hand we have detailed the process of deriving the Web application supporting the business processes defined in the BPM. This process is performed in two steps. First of all, we derive from the BPM the Navigational and Presentation model which represent in an abstract way the elements that make up the web pages of the web application. Then, in a second stage we instantiate these models into a particular Web framework, Tapestry in this case. As a result, a ready to run Web application supporting the execution of the originally specified business processes is built. 


\section{Chapter 10}

\section{Tool Support}

Models in software engineering were initially used for documentation purposes. These were relegated to play a secondary role within the software development process. However, approaches such as the Model Driven Development (MDD) changed that reality. This approach brought models as first class citizens during the software development process. The main advantage of this approach was that software applications could be automatically generated from models. In addition, nowadays, the maturity reached by technologies and standards allow turning the MDD approach into reality. Examples of these mature tools are the Eclipse Modelling Project (EMP) or the Domain-Specific Language tools from Microsoft.

This chapter presents the BIZZY tool which has been developed in the context of the EMP where a set of Eclipse plugin (model editors and model transformation languages) have been combined to completely support the development process proposed in this thesis. On the one hand, the included model editors allow developers creating and manipulating the set of models defined in this work (Structural, Services, Business Process Navigation and 
Presentation Models). On the other hand, model transformation languages have been used to implement model-to-model (M2M) and model-to-text (M2T) transformations that allow obtaining respectively other models (specifically the Navigational model) and their equivalent representation but this time in terms of a technological representation.

The chapter has been organized as follows. First, section 10.1 takes up again the development process presented in chapter 4 to detail the tools used in each step of the process. Then, in section 10.2, the development process is presented step by step by detailing the tools used and the artefacts consumed and generated in each step. Section 10.3 presents how the artefacts obtained after the execution of the implemented model transformations are deployed to a Web server. Finally, this chapter is concluded in section 10.4.

\subsection{Tool Overview}

The generation of BP-driven Web applications is performed by using different model editors and model transformations along the different steps defined in the development process. According with the development process presented in chapter 4, this process is divided into two main steps which relate to system specification (problem space) and system generation (solution space). The coordinated use of these model editors and model transformations allows generating the Web application according to the models specified at the problem space. Figure 10.1 shows the tool support 
provided for each of the steps defined in the process. In this figure, when a model transformation is performed, this has been indicated by means of a gear image, which includes in the type of transformation performed (M2M, M2T or both).

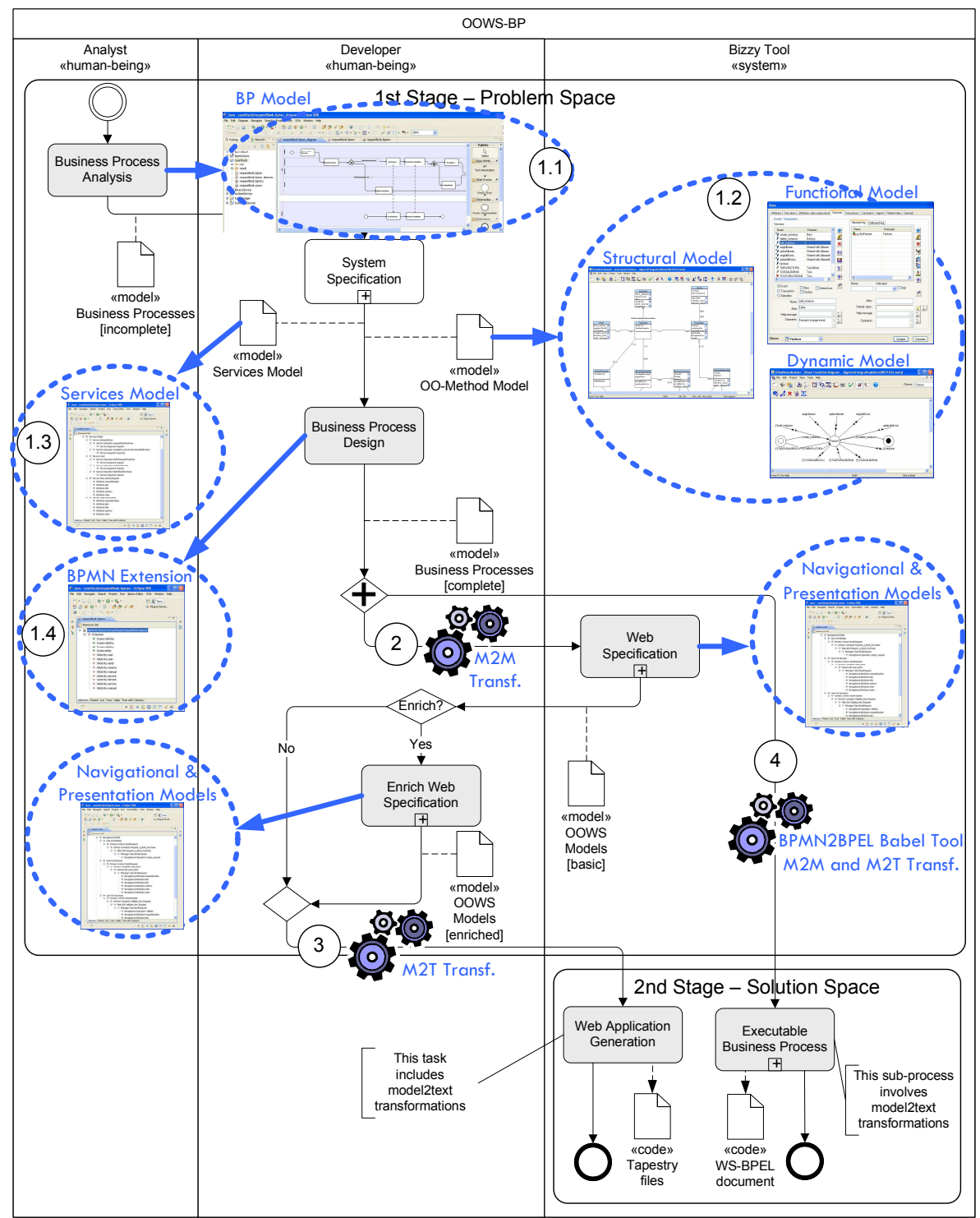

Figure 10.1 Tool Support for the OOWS-BP Development Process 
Model editors and transformations used along the development process have been built from a set of tools included in the Eclipse project, specifically from the Eclipse Modeling Framework ${ }^{46}$ (EMF). EMF includes tools for the generation, edition and serialization of models conforming to Ecore metamodels (an implementation of the OMG's Essential MOF to represent metamodels). In the BIZZY tool, all the used metamodels (BPMN, WS-BPEL and OOWS) are represented as Ecore metamodels. In some cases, the Ecore metamodels were built from the corresponding XML Schemas (EMF permits the generation of Ecore metamodels from XML Schemas). In other cases, a new Ecore metamodel had to be built.

With regard to model editors, most of the model editors included in the BIZZY tool are provided as a tree-based EMF editor. The only graphical editor included in the tool is the BPMN Modeller ${ }^{47}$ that has been developed in the SOA Tools Platform (STP).

With regard to model transformations, two different languages have been used. On the one hand, the Atlas Transformation Language $^{48}$ (ATL) was used to deal with M2M transformations. Specifically, we have implemented in this language the transformations that allow transforming (1) the Business Process Model (BPM) into the corresponding OOWS Navigational Model

\footnotetext{
46 http://www.eclipse.org/modeling/emf/

47 http://www.eclipse.org/stp/bpmn/

$48 \mathrm{http}: / /$ www.eclipse.org/m2m/atl/
} 
and (2) the BPM into BP format accepted by the BPMN2BPEL Babel tool. On the other hand, the MOFScript Language was used to deal with M2M transformations. Specifically, the transformations that have been implemented in this language allow generating (1) the interface (WSDL file) and the data types (XSD file) used by the Web service represented by the WS-BPEL BP and (2) the Web applications in terms of a Web Framework (in particular to the Tapestry Web Framework).

Finally, in addition to these Eclipse-based model editors and transformations, a Java tool has been used to perform the step that partially builds the executable WS-BPEL document. This tool is the Babel BPMN2BPEL ${ }^{49}$ tool. Its role is performing the transformation between BPMN diagrams into WS-BPEL definitions.

The following section presents in detail each of the steps of the development process.

\subsection{From a BP Specification to a Web Application: Step by Step}

According to Figure 10.1, the steps that have to be performed to obtain a Web application supporting BPs are the following:

${ }^{49} \mathrm{http}: / /$ www.bpm.fit.qut.edu.au/projects/babel/tools/ 
1. System Specification: Specify the different aspects of the system (Structure, behaviour) by means of the corresponding editors.

2. Generation of the Web System Navigation: Execute a M2M transformation that generates the Navigational model required to support the execution of the BPs defined in step 1. This is an ATL transformation that is executed from the ATL plug-in for Eclipse.

3. Generation of the Web System GUI: Execute a set of M2T transformations that generate the Web application in terms of the Tapestry Web framework. These transformations are implemented in MOFScript and are executed from the MOFScript tool developed as an Eclipse plug-in.

4. Generation of WS-BPEL executable BPs: Execute a set of M2M, M2T and the BPMN2BPEL Babel tool to obtain an executable definition of the BPs defined in step 1 . Depending on the case, these transformations have been implemented in ATL and MOFScript.

The following subsections present each of these steps by means of the "Book Request Purchase" long-running BP case study (a complete description is provided in Appendix C). For each step we detail its sub steps (if any), the required models, the tool used and the produced artefacts. 


\subsubsection{Step 1: System Specification}

Along this step the system is modelled by the developer in terms of the models proposed by the method. These models represent the system in an independent technology manner. This step is made up in turn of four steps and the order in which these are built can vary from case to case. However, since we start from a BP specification, we propose the following order:

1.1 Build an initial BP definition. This step is performed by means of the BPMN Modeller included in the STP (see Figure 10.2). This step constitutes the first attempt to build the Business Process Model (BPM) defined in the proposal.

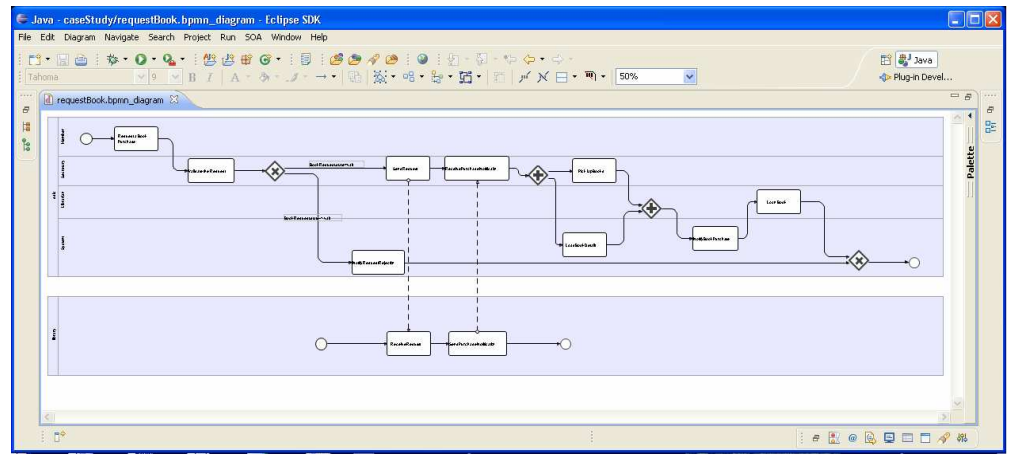

Figure 10.2 STP BPMN Modeller

1.2 Build the Structural, Dynamic and Functional models defined by the OO-Method approach. This step is performed by means of the OLIVANOVA Modeller. 

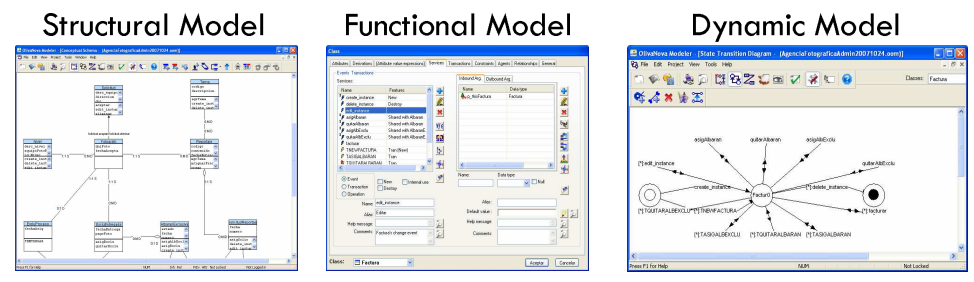

Figure 10.3 Different Editors provided by the OLIVANOVA Modeller

These models are translated into code by means of the OLIVANOVA Transformation Engines. The integration of the code generated by these engines was solved in (Valverde et al., 2007). In this work, authors developed a Business Façade interface which exposes a set of methods that are redefined according to the underlying business logic. This façade is used to build and send XML messages to the components that encapsulate the business logic specified in the OLIVANOVA Modeller.

1.3 Build the Services Model included in the OO-Method approach. This model allows specifying the functionality that is provided by external partners at the modelling level. Nowadays, this model is built manually from the WSDL interface provided by external partners. This is performed by means of the tree-based EMF editor generated for the Services Model. 


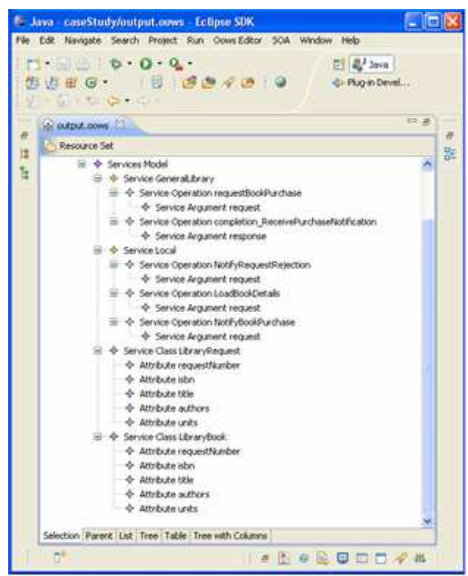

Figure 10.4 Tree-based EMF editor for the Services Model

1.4 Complete the BP definition that has been built in step 1.1 according the BPMN extension presented in chapter 5. This extension allows specifying the functionality that supports each of the BP activities and the behaviour of the human roles involved in the BP. This completion is performed by means of a three-based EMF editor which extends some of the elements defined in the original BPMN notation (see Figure 10.5). After this step, the BPM is completed and can be used to produce different artefacts. 


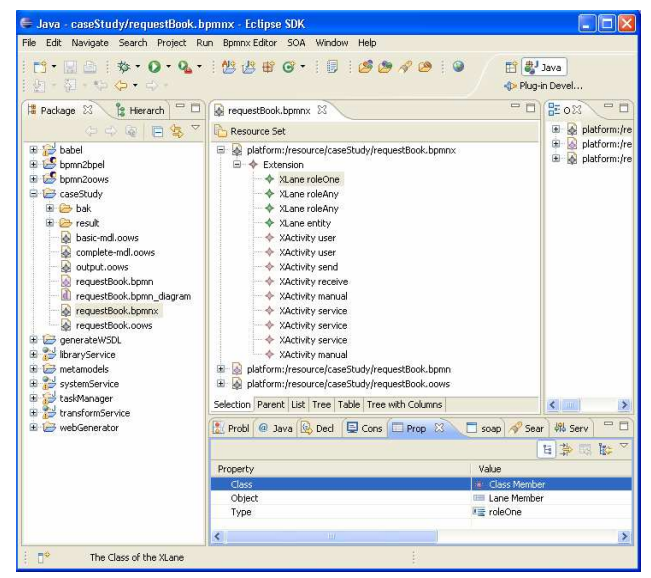

Figure 10.5 Tree-based EMF editor for the Extension defined over the BPMN notation

\subsubsection{Step 2: Generation of the Web System Navigation}

The result that is obtained in this step is the Navigational Model corresponding to the system specified in the previous step. This model represents the Navigation of the Web application in a technology independent manner. The Navigational Model is generated in one step; however, optionally, this model can be improved manually in a second step (step 2.2):

2.1 The former step is performed automatically by means of a M2M transformation (bpmn2oows). This transformation generates, from the BPs specified in the BPM (steps 1.1 and 1.4), the Navigational model required to support the execution of these BPs. This transformation has been implemented as an ATL transformation and is executed 
from the ATL plug-in for Eclipse. This transformation ${ }^{50}$ has been previously presented in chapter 9 . Figure 10.6 shows the input and output artefacts of the "bpmn2oows" transformation.

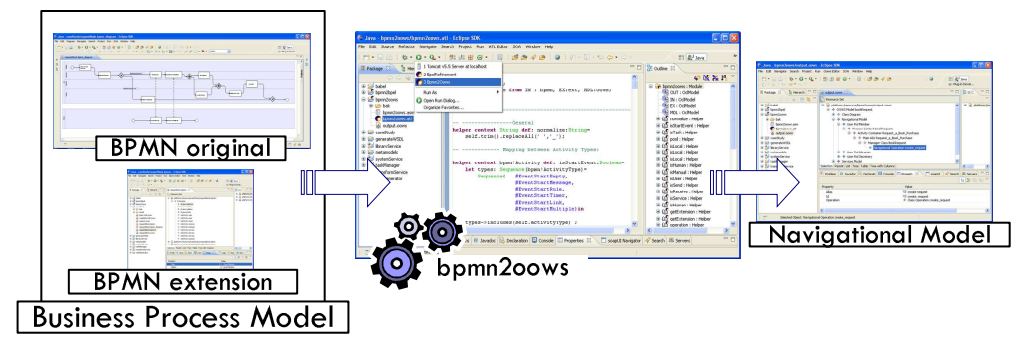

Figure 10.6 From BPM to OOWS Navigational Model

2.2 Although the Navigational model obtained previously in step 2.1 it already supports the execution of the defined BPs, this model can be manually enriched to include information to improve the final generated Web application. Specifically, in this step we can include complementary information that is kept in the system to help users in completing their tasks. This step is performed manually by the developer and it is done by means of the tree-based EMF editor built for the Navigational model.

\subsubsection{Step 3: Generation of the Web System GUI}

Once the whole system is specified at the problem space, we can proceed with the execution of M2T transformations that generate the Web application in terms of the Tapestry Web framework.
50
Available
on-line
at

http://www.dsic.upv.es/ vtorres/thesis/appendixes/appendixG 
These transformations require all the previously built models and they produce the different files required by the Tapestry Web framework which are Java, page and html files. These transformations $^{51}$ are implemented in MOFScript and are executed form the MOFScript tool developed as an Eclipse plug-in.

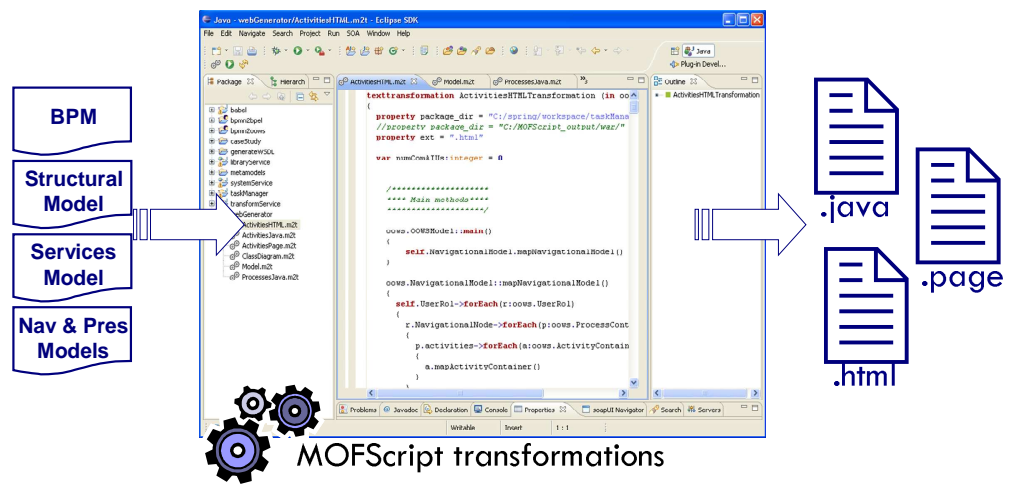

Figure 10.7 M2T Transformations

After the execution of these transformations, a Tapestry Web application is generated. The Web application is made up of files that are dependent of the domain (files that are necessarily generated for each Web application) and independent of the domain (files that are always the same independently of the specific Web application). To differentiate these types, the dependent files are always contained within a folder named "gen" (see Figure 10.9 and Figure 10.9). According to this criterion, as Figure 10.8 shows, the structure of this application looks like the same as a Java Web application. Specifically, the taskManager

51 Available on-line at

http://www.dsic.upv.es/ vtorres/thesis/appendixes/appendixH 
folder (which corresponds to the "Book Request Purchase" case study) is made up of three folders (the "gen", "images" and "WEBINF") and a set of html, page and css files.

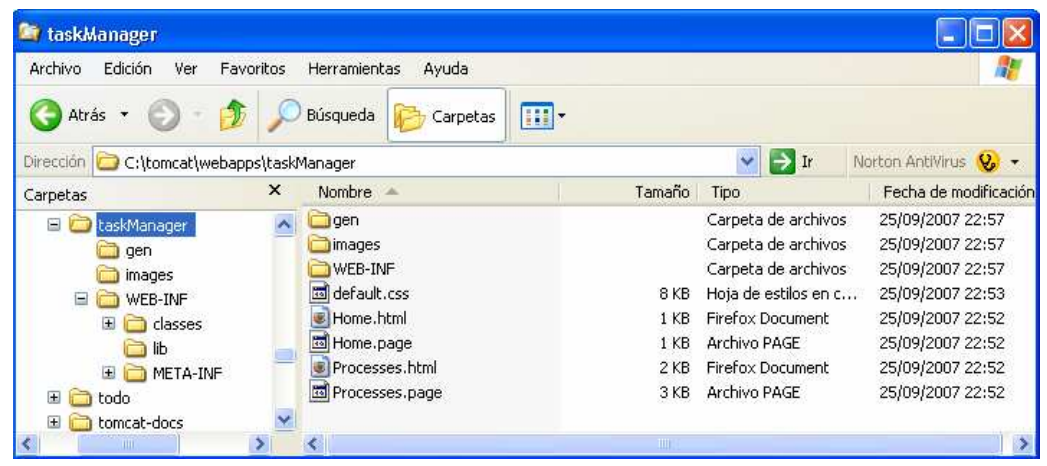

Figure 10.8 Web application folder within the TOMCAT Web server

The "gen" folder contains the html templates of the different Web pages of the application and, when applies, the associated page files (remember that these files specify the Tapestry components used in the templates).

The html and page files contained in this folder are files independent of the domain and correspond to the initial web page when the Web application is started (Home.html) and the navigation provided to a specific user regarding to BPs (Processes.html).

The default.css file is the Cascading Style Sheet used in the Web application which defines colours, fonts, layout and other aspects of the presentation of the web pages. The images folder contains the application's images used by the Web pages. 


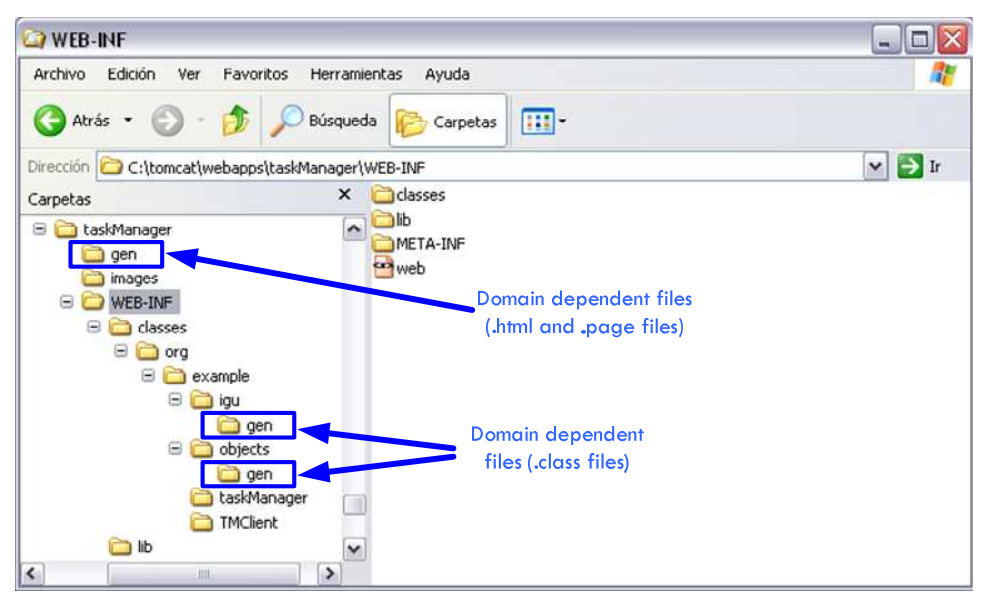

Figure 10.9 WEB-INF Folder Structure

Finally, the WEB-INF directory contains all the inner workings of a Java Web application. This directory contains three subfolders which are:

- classes folder: This folder contains the Java classes that implement the functionality of the Web application.

- lib folder: This folder contains all the libraries required by the Web application. Specifically, the Tapestry libraries are placed in this folder.

- META-INF folder: This directory contains the manifest file which describes the package that is created from the Web application.

- web.xml file: This file is the deployment descriptor which serves to tell the Web server all of the most important details about the Web application and its configuration. 


\subsubsection{Step 4: Generation of WS-BPEL executable BPs}

The generation of the WS-BPEL BP is performed in four steps. The use of the BPMN2BPEL Babel tool to generate a partial definition of the WS-BPEL document forces us to prepare BP definitions (those modelled in the BPM) according to the format required by this tool. Therefore, the steps required to obtain a complete WS-BPEL document are the following:

4.1 Execute the bpmn2babel M2M transformation to prepare the BPs defined in step 1.1 into the format accepted by the BPMN2BPEL Babel tool. This transformation ${ }^{52}$ has been implemented in ATL and it is executed from the ATL plugin for Eclipse. Figure 10.10 shows the models consumed and generated by the bpmn2babel transformation.

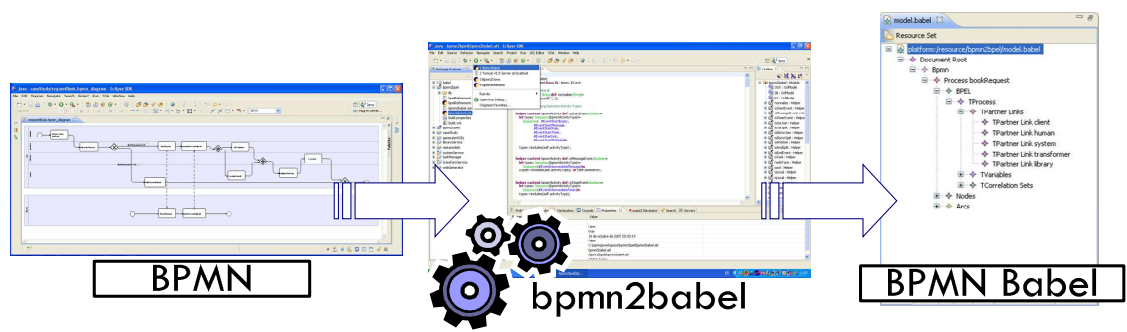

Figure 10.10 M2M transformation to prepare BP definitions according to the Babel tool

4.2 Execute the BPMN2BPEL Babel tool to obtain a partial definition of the executable BP defined in step 1.1. This 
step is automated by means of an Ant script which defines targets to invoke the Babel Java tool. Figure 10.11 shows the consumed and generated models after the execution of the BPMN2BPEL Babel tool.

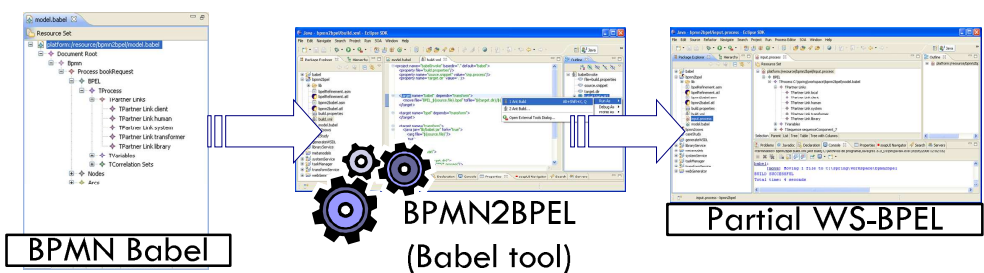

Figure 10.11 Generation of a partial WS-BPEL BP

4.3 Execute the bpelRefinement M2M transformation which completes the WS-BPEL document obtained in step 4.2. As Figure 10.12 shows, this transformation uses the Structural, Services and Business Process Model to complete the WS-BPEL document generated previously in step 4.2. This transformation ${ }^{53}$ has been implemented in ATL and it is executed from the ATL plug-in for Eclipse.

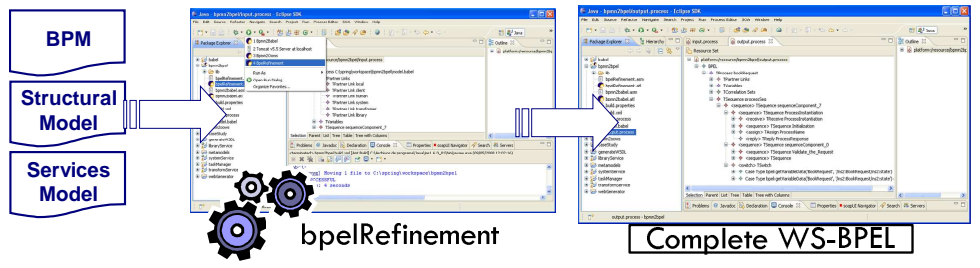

Figure 10.12 Generation of a complete WS-BPEL BP 
4.4 Execute the M2T transformations that generate the interface and the data type files corresponding to the WSBPEL obtained previously. These files are necessary since the WS-BPEL BP is in turn a Web service. These files are generated from the Structural, Services and Business Process Models. These transformations ${ }^{54}$ have been implemented in MOFScript and it is executed from the MOFScript plug-in for Eclipse.

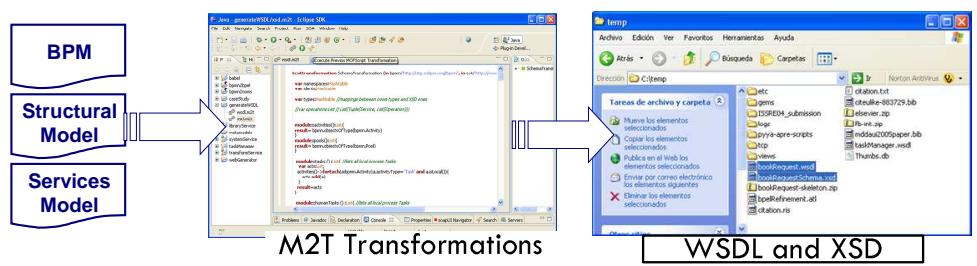

Figure 10.13 Generation of the Interface and Data types used by the WSBPEL BP

When the WS-BPEL document and the WSDL and XSD files have been generated we can bring them into the ActiveBPEL Eclipse environment to proceed with the deployment of the WS-BPEL BP (see section 10.3.2).

\subsection{Deployment}

Once the system has been obtained in terms of an implementation technology, this has to be deployed in a Web server. Both the Web applications and the WS-BPEL are deployed in a Web server. Then, when the server is started both applications will be served

54 Available on-line at

http://www.dsic.upv.es/ vtorres/thesis/appendixes/appendixE 
by the server. The following subsections explain how the interface of the Web application and the WS-BPEL BP are deployed in the Web server.

\subsubsection{Web Application Deployment}

Before deploying the Web application in a Web server it is necessary to compile all the Java files generated in step 3. These tasks (compilation and deployment) have been automated in an Ant script (Code excerpt 10.1). After the execution of these tasks, a Web application with the structure shown in section 10.2.3 is created.

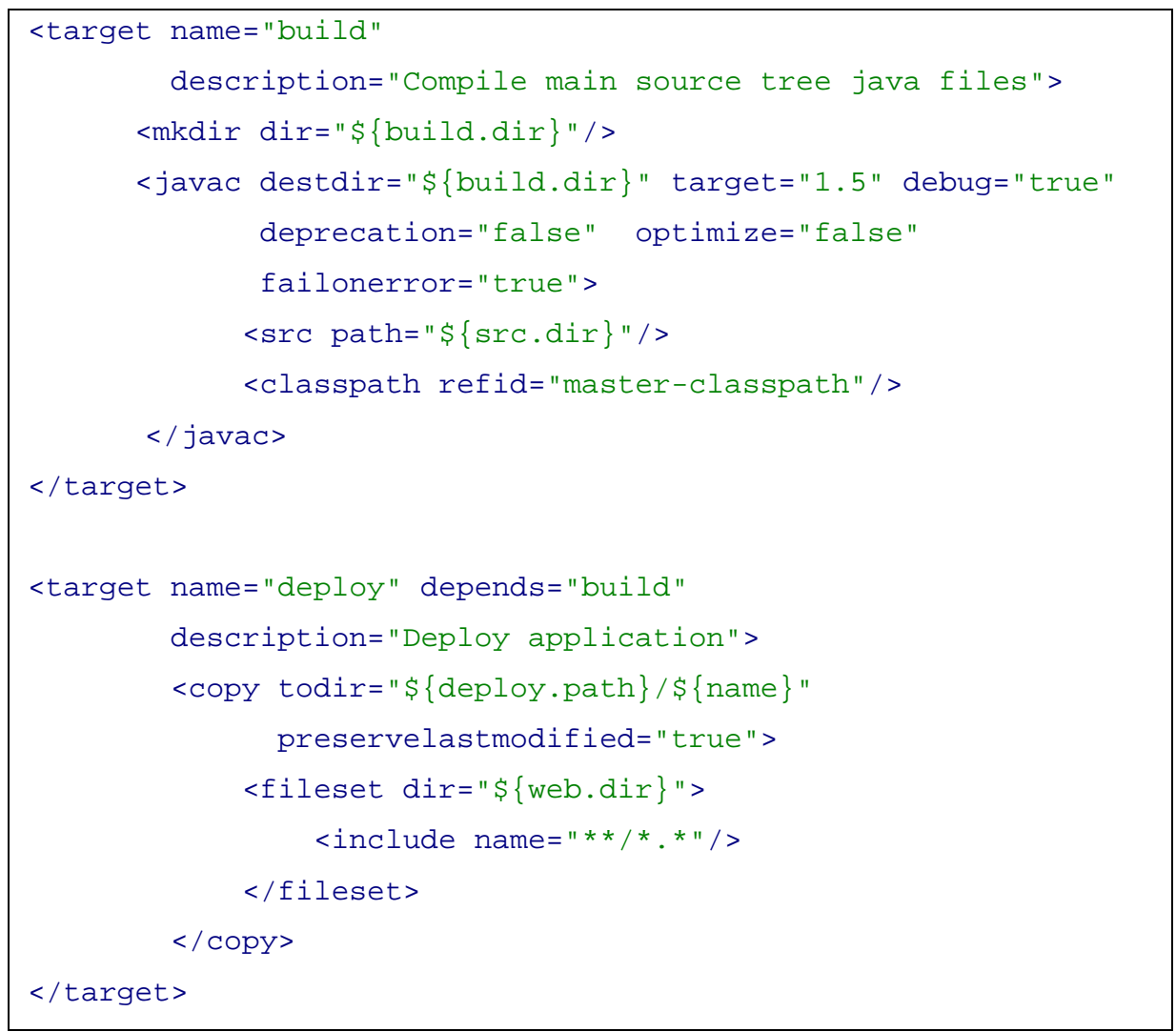

Code excerpt 10.1 Build.xml excerpt 


\subsubsection{WS-BPEL Deployment}

The deployment of a WS-BPEL BP to a Web server is performed from the ActiveBPEL Eclipse tool (see Figure 10.14). The steps that have to be done to proceed with the deployment are the following:

- Import a skeleton project for ActiveBPEL. This skeleton includes:

- A WSDL folder which contains the wsdl files of the external partners (in this case this corresponds to the Central Library partner) and the task manager (this service is always used by the generated WSBPEL process to deal with the human limitation found in the WS-BPEL version used in this work (version 1.1)).

- PDD (Process Deployment Description file): This file indicates the urls where the different services involved in the WS-BPEL are located.

- Copy the automatically generated .bpel file (see section 10.2.4) into the imported project (bookRequest.bpel in Figure 10.14).

- Copy the generated wsdl and xsd files (10.2.4) into the imported project (bookRequest.wsdl and bookRequestSchema.xsd in Figure 10.14).

- Export the PDD file as a Business Process Archive (.bpr) file. As a result, an archive deployment file is created (bookRequest.bprd file in Figure 10.14). 


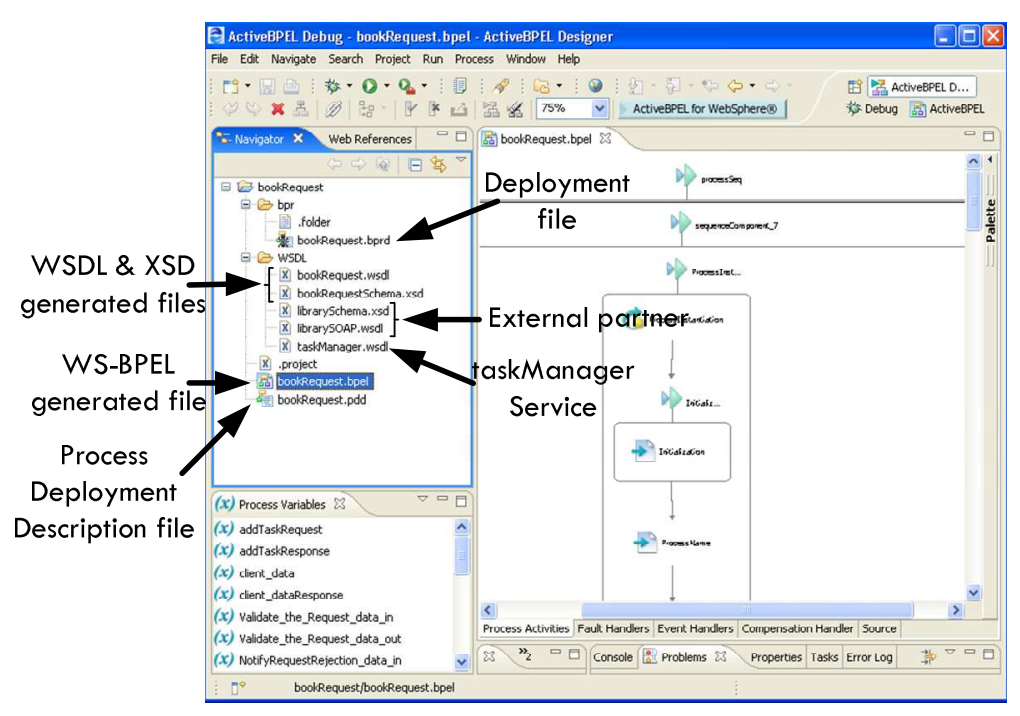

Figure 10.14 ActiveBPEL prepared environment

The WS-BPEL deployed process can be tested from the "BPEL Administrative Console" which can be reached from the "Administrative Servlets" provided with the activeBPEL engine. This console allows checking that the deployment was successful.

After the deployment of the WS-BPEL processes, the active-bpel folder shown in Figure 10.15 is created.

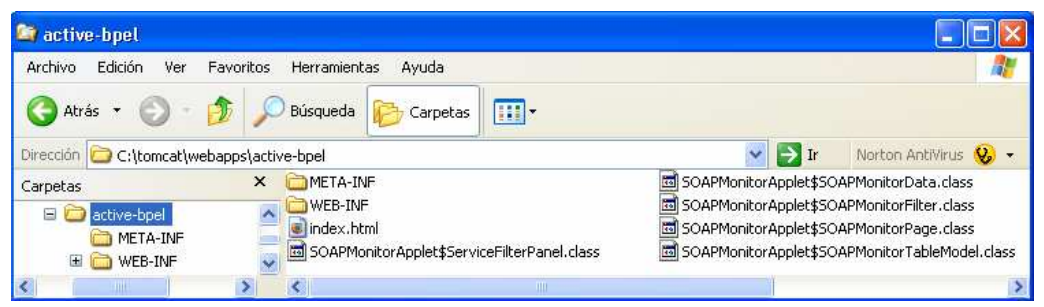

Figure 10.15 Active-bpel folder within the TOMCAT Web server 


\subsection{Conclusions}

In this chapter we have presented the set of model editors and transformations used along the development process to generate a BP-driven Web application. We have detailed for each step of the process the artefacts consumed and generated and the tool used to complete each step.

Model editors have been used to specify the different aspects that characterize BP-driven Web applications. Most of them have been provided as a tree-based EMF editor. On the other hand, model transformations have been implemented to obtain (1) a Java Web application and (2) the set of WS-BPEL BP according to the system specified at the modelling level. To implement model transformations we have used two different languages which are ATL and MOFScript. These languages have been used to implement model-to-model and model-to-text transformations respectively.

Additionally, we have explained how the generated artefacts (Web application in terms of the Tapestry Web Framework and WSBPEL processes) are deployed in a Web server. On the one hand, the Web application has been generated according to the folder structure required for a Java Web application. On the other hand, the WS-BPEL process is automatically deployed to the Web server through the ActiveBPEL Eclipse environment. 



\section{Chapter 11}

\section{Conclusions \& Further Work}

In this thesis, we have presented a methodological solution to cope with the automatic generation of BP-driven Web applications. To achieve this goal, we have developed a solution within the Web engineering field that is inspired on the architecture of the Business Process Management Solutions (BPMS). Furthermore, the proposed solution is also based on the Model Driven Engineering field, where the definition of model transformations allows us to move the system that is specified at the modelling level into a specific implementation technology.

This last chapter introduces the conclusions of the work developed in this thesis. First, we present the main contributions to the Web engineering community. Then, we outline the ongoing and future work that has emerged from this thesis. Finally, we present the publications that have been produced throughout the development of this work.

\subsection{Main Contributions}

The main contributions of this work are: 
- We have defined a complete process to carry out the development of BP-driven Web applications. This process extends from the modelling phase (the phase where the system is represented in terms of a set of models) through the generation phase (the phase that applies a set of transformation rules to obtain the executable artefacts).

- At the modelling level, we have defined a set of abstractions that represent navigational and presentation properties found during the business process (BP) execution. These abstractions have been introduced into the OOWS Web Engineering method.

- We have modified the architecture of the generated Web applications to properly handle BPs. As a result, we have introduced a process engine into the architecture of these systems. This process engine allows the construction of more lightweight navigational models, where the process flow is maintained inside the process definition.

- Based on the MDE, we have defined a set of model transformations to obtain (1) executable process definitions expressed in the WS-BPEL language and (2) the set of files (.java, .html and .page) necessary to deploy an application in terms of a Web framework, specifically the Tapestry Web framework ${ }^{55}$.

- We have developed a tool (the BIZZY tool) that implements the ideas presented in this work. The tool has been developed applying the latest trends in the MDE field. It

55 http://tapestry.apache.org/ 
has been built using tools that are included in the Eclipse development environment (the Eclipse Modeling project and the SOA Tool Platform project) and the BPMN2BPEL Java tool ${ }^{56}$. Tools such as ATL and MOFScript have been used to implement the transformations defined in the proposal and EMF has been used to manipulate the models defined in the method. Finally, the BPMN editor from the STP Project has been used to model BPs defined in the Business Process Model included in the proposal.

\subsection{Further Work}

The research presented here is not a closed work and there are several interesting directions that can be taken to provide the proposal with a wider spectrum of application. The following list summarizes the research activities that are planned to continue this work.

- Business Process Semantics. We propose to present semantic information related to the BPs supported by our generated Web applications. This extra information will provide potential consumers with a more complete description of the offered functionality. To achieve this goal, these services must be described in semantic languages such as OWL-S (Burstein et al., 2004) or WSMO (Roman et al., 2005). In keeping with the MDE trends applied in this work, we should define mappings that allow

\footnotetext{
${ }^{56} \mathrm{http}: / /$ www.bpm.fit.qut.edu.au/projects/babel/tools/
} 
us to translate the BPs represented in our systems into any semantic language.

- Business Rules. Web service technology has propitiated the existence of multiple service providers offering similar services. Since performing an automatic selection based on a set of variables is very important, we plan to study how these business rules can be defined within the method following the MDE trends on which this work is based.

- Assisting the User during Business Process Definition. It is of utmost importance to ensure that the developed system satisfies user requirements. In order for the system to be correct and valid and ensure that it corresponds to the user requirements it is necessary to validate the specification of the system at the problem space prior to its construction. To do this, we plan to integrate a mechanism at the modelling level that assists the user during the BP specification phase. This mechanism is based on a recommender system that can validate the semantics of the BP (Hornung et al., 2007).

- Graphical Editor. The current state of the BIZZY tool does not provide a graphical editor for handling the Navigational and Presentation models. Therefore, to improve the developer's experience, we plan to complete the tool with graphical editors. Since the tool has been developed within the Eclipse environment, the graphical editor will be developed using the Eclipse Graphical Modeling Framework (GMF), which allows visualizing models in a graphical manner. 
- Automatic generation of Web service adapters. Based on research works such as (Benatallah et al., 2005), we want to endow the tool with a mechanism for generating the transformation service that is in charge of mapping the data types used in the WS-BPEL process with the data types that are used by external partners.

- Content Adaptation to Target Devices. We are aware of the broad range of devices that can be used to access the WWW. These include desktop computers, PDAs or mobile phones among others. However, each device defines a set of characteristics (i.e., small size, voice recognition capabilities, etc.) that must necessarily be taken into account when accessing Web applications. We have started to deal with these issues by defining a model that allows us to represent the characteristics of these devices to see how they affect the data and functionality provided by the accessed systems. This preliminary work has already been done in (Torres et al., 2007). Extensions to this work will improve the original proposal.

\subsection{Publications}

The work developed in this thesis has been published in the following book chapters, conferences and workshops:

- Victoria Torres, Vicente Pelechano, Marta Ruiz, Pedro Valderas, "A Model Driven Approach for the Integration of External Functionality in Web Applications. The Travel 
Agency System". Workshop on Model-driven Web Engineering (MDWE), July, 2005, Sydney (Australia), pp. 1-11

- Victoria Torres, Javier Muñoz, Vicente Pelechano "A Model-Driven Method for the Integration of Web Applications". LA-WEB, October, 2005, Buenos Aires (Argentina), pp. 32-41

- Victoria Torres, Ricardo Quintero, Marta Ruiz, Vicente Pelechano. "Towards the Integration of Data and Functionality in Web Applications. A Model Driven Approach", Conference on Advanced Information Systems Engineering Forum (CAiSE Forum), June, 2005, Porto (Portugal), pp. 33-38

- Victoria Torres, Vicente Pelechano. "Building business process driven web applications". In Dustdar, S., Fiadeiro, J.L., Sheth, A.P., eds.: Business Process Management. Volume 4102 of Lecture Notes in Computer Science., Springer (2006) 322-337

- Victoria Torres, Vicente Pelechano, Pau Giner. "Generación de Aplicaciones Web basadas en Procesos de Negocio mediante Transformación de Modelos". XI Jornadas de Ingeniería del Software (JISBD), 2006, pp. $443-452$ 
- Victoria Torres, Pau Giner, Vicente Pelechano. Eder, J.; Tomassen, S. L.; Opdahl, A. L. \& Sindre, G. (ed.) "Modeling Ubiquitous Business Process Driven Applications'. Conference on Advanced Information Systems Engineering Forum (CAiSE Forum), CEURWS.org, 2007, 247

- Pau Giner, Victoria Torres, Vicente Pelechano, "Bridging the Gap between BPMN and WS-BPEL. M2M Transformations in Practice". Proc. of the 3rd International Workshop on Model-Driven Web Engineering (MDWE), July, 2007, Como (Italy)

- Victoria Torres, Vicente Pelechano and Pau Giner, "Building business process driven web applications based on the service oriented paradigm," ERCIM News, vol. 70, pp. 54-55, July 2007.

- Victoria Torres, Pau Giner, Vicente Pelechano, "Web Application Development Focused on BP Specifications", Taller sobre Procesos de Negocio e Ingeniería del Software (PNIS), September, 2007, Zaragoza (Spain), pp. 1 - 7

- Pau Giner, Victoria Torres, Vicente Pelechano. "Generation of Business Process based Web Applications" (Demo). XII Jornadas de Ingeniería del Software y Bases de Datos (JISBD), September 2007, Zaragoza (Spain). 
- Victoria Torres, Joan Fons, Vicente Pelechano. "Building Usable Business Process Driven Web Applications". In Calero, C.; Moraga, M. \&A. \& Piattini, M. (ed.) Handbook of Research on Web Information Systems Quality Building Usable Business Process Driven Web Applications IGI Global, 2008, pp. 247-265.

- Joan Fons, Vicente Pelechano, Oscar Pastor, Pedro Valderas, Victoria Torres. "Applying the OOWS ModelDriven Approach for Developing Web Applications. The Internet Movie Database Case Study'. In Rossi, G.; Pastor, O. S. D. O. L. (ed.) Web Engineering: Modelling and Implementing Web Applications Springer London, 2008, pp. $65-108$

The following table summarizes the publications presented above based on the place where these have been published.

Table 2 Publication summary

\begin{tabular}{|c|c|c|}
\hline & Place & $\#$ \\
\hline Book chapters & Springer(1), IGI Globlal (1) & 2 \\
\hline International & BPM(1), CAiSE Forum (2), & \\
Conferences & MDWE(2), & 6 \\
& LA-WEB(1), & \\
\hline National Conferences & JISBD (2), PNIS (1) & 3 \\
\hline Journal & ERCIM (1) & 1 \\
\hline & & 12 \\
\hline
\end{tabular}




\section{Appendix A}

\section{Lybrary4U Case Study}

This appendix presents the Lybrary4U case study focusing on those parts related with pure navigation. Then, appendixes B and $\mathrm{C}$ will extend this part to provide support for short and longrunning Business Processes.

This appendix is structured as follows. First of all, a textual description of the case study is presented. Then, based on this description, sections A.2 and A.3 present the domain represented in the Structural and the Services models respectively. Once these models are completed, sections A.4 and A.5 develop the User, Navigation and Presentation models following the OOWS approach. These three models define (1) the kind of users that are going to use the application (and the inheritance relationships between them), (2) the visibility over the system for each kind of user defined in the user model and (3) the organization of the contents defined in the Navigational model.

\section{A.1 Description}

The Library $4 \mathrm{U}$ case study is a web application developed basically to support the information system related with a library. However, the library also provides its members with an on-line book store 
service that sends members the shopped books to the address provided by the member.

Therefore, it is necessary to provide mechanisms that allow users looking for information contained in the system (for the shake of simplicity it has been reduced to Authors and Books). Moreover, this search functional is extended depending on the privileges granted to specific users. For this reason, there are three levels of privileges, which are Members, Librarians and Secretary Staff. Members reference the group of users that can borrow books from the library and buy books from the on-line store. The Librarian group characterizes the users that make effective the loan of a particular book to a particular member. Finally, the Secretary Staff refers to users that validate proposals made by members (i.e. including into the library catalogue a particular book).

\section{A.2 Structural Model}

According to the two main objectives of the Library4U system (books loan and books sale), Figure 12.1 shows the class diagram that represents the structure of the system in terms of classes and relationships. On the one hand, classes such as copy, loan or member are clearly associated to the books loan objective. On the other hand, classes such as ShoppingCart, Offer or itemProduct are clearly associated to the books sale objective. In addition, the class Book represents the main class of this diagram being connected with most of the remainder classes. 


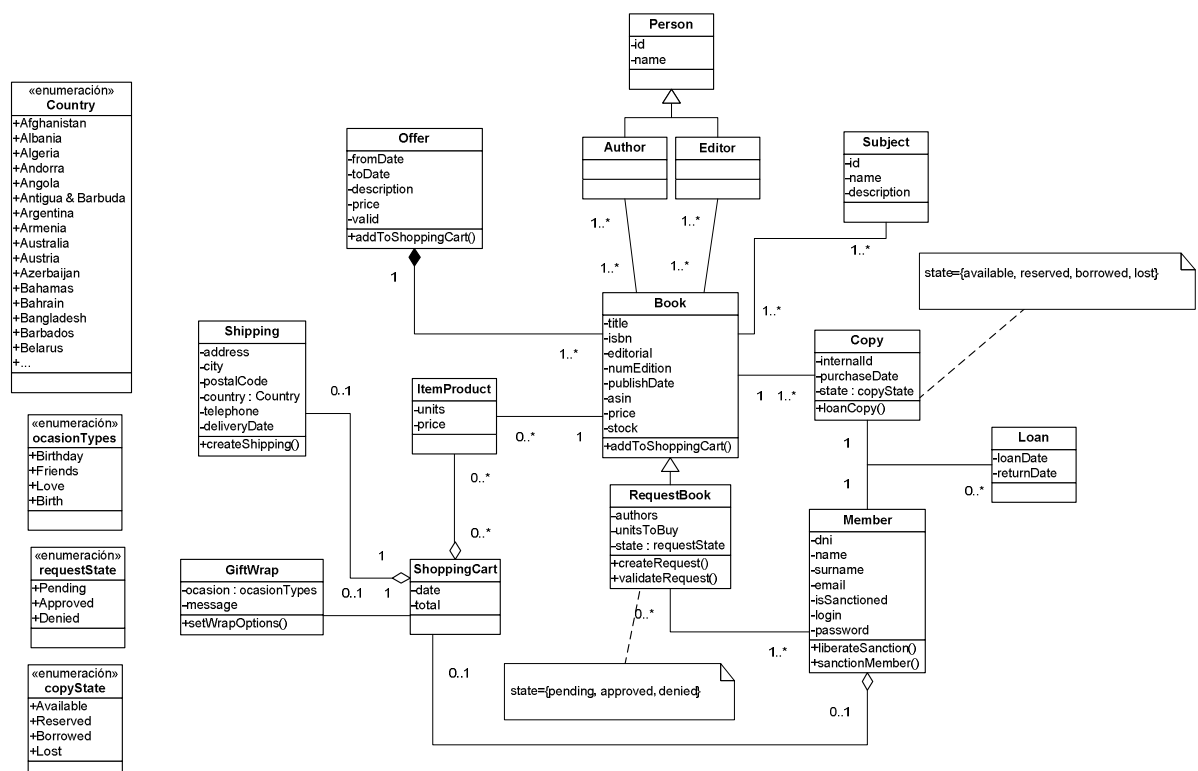

Figure 12.1 Library4U Class Diagram

The class Book includes the following attributes: title, isbn, editorial, number of edition, year of publication, price, stock and asin (Amazon identifier number). This latter identifier number is kept as book data since is going to be used for retrieving data from the Amazon Web Service to complement the data that is kept in the local Library $4 \mathrm{U}$ database. Moreover, a book has been written by many authors (represented by means of many-to-many relationship between Book and Author classes) and it is catalogued in many subjects (represented by means of many-tomany relationship between Book and Subjects classes). The library keeps several copies of the same book, being this represented by the copy class (Copy) and the one-to-many relationship. Then, book copies are loan to members in a particular date, which is represented by the Loan class associated 
to the relationship between the Copy and Member classes. The loanCopy() operation defined in the Copy class changes the state of the copy and creates a loan for the specified member, establishing the loan and return dates of the loan. Moreover, members can propose the purchase of particular books. This is represented by the specialized RequestBook class. When the book is finally bought this is promoted to the Book class.

Regarding the on-line book store, the class diagram includes the Shopping Cart class. This class is associated with just one member (in case the member has already logged in the system). Moreover, the Shopping Cart class is at list composed of one item (itemProduct), which is associated with a book. Finally, the shopping can be shipped to a particular address (Shipping) and gift wrapped for a special occasion (GiftWrap).

\section{A.3 Services Model}

In order to complement the data and functionality provided by the local system (whose structure has been represented in the Structural Model), the Library4U web application makes use of external services. In particular we are going to use the Amazon Web Service to enrich the information kept in the local system about books.

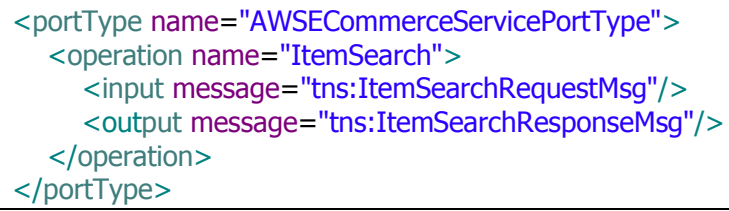

Code excerpt 12.1 WSDL Port type definition 
From the set of services provided by the Amazon Web Service we are going to make use of the ItemSearch operation (see Code excerpt 12.1). This operation returns items that satisfy the specified search criteria. We are going to provide as search criteria the book title kept in our system. This corresponds to one of the elements defined in the request type of the ItemSearch operation (see Code excerpt 12.2).

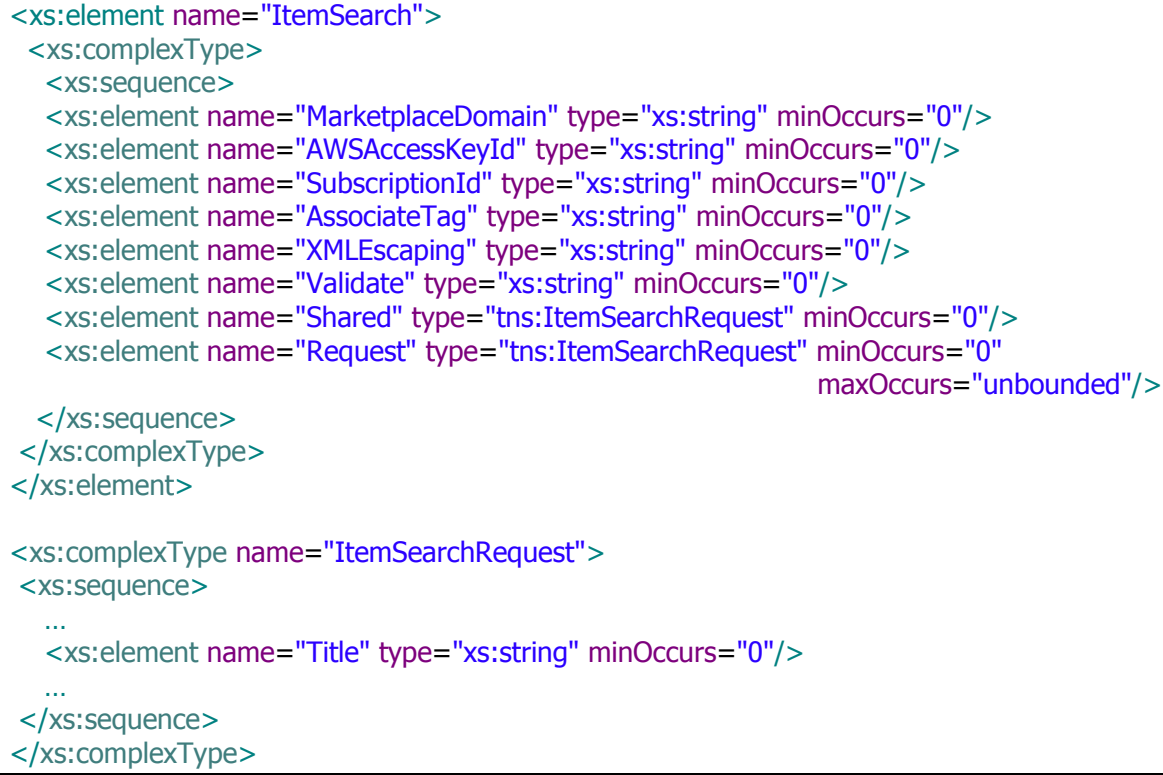

Code excerpt 12.2 ItemSearch Data Type

\section{A.4 User Model}

The kinds of users that can interact with the system are four, which are categorized into two groups, one related to users that do not have to be logged into the system (depicted in Figure 12.2 with the "?" symbol) and another related to users that have to be logged into the system (depicted in Figure 12.2 with the lock symbol). The Anonymous user type has been included within the first group. 
Normally, the visibility of the system for this kind of users is quite limited. Within the second group the Member, Librarian and Secretary user types have been defined.

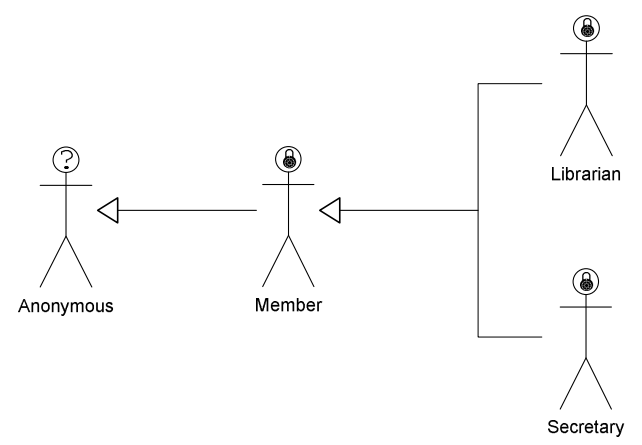

Figure 12.2 User Model

Moreover, all these kinds of users are connected through inheritance relationships which allow propagating characteristics (in particular navigational characteristics) from parents to children.

The following section presents the Navigational Model defined for each kind of user depending on their responsibilities.

\section{A.5 Anonymous Navigational and Presentation Model}

This section present the Navigational and Presentation Models defined for the Anonymous type of user identified in the User Model. Attending to the two steps in which the Navigational model is built first of all a global description of the navigation is performed (the Authoring-in-the-large). Then, a detailed description of the navigation is performed (Authoring-in-thesmall). 


\section{A.5.1 Authoring-in-the-large}

The Navigational Map for the Anonymous user is made up of four navigational contexts providing each of them a different view over the class diagram (see Figure 12.3).

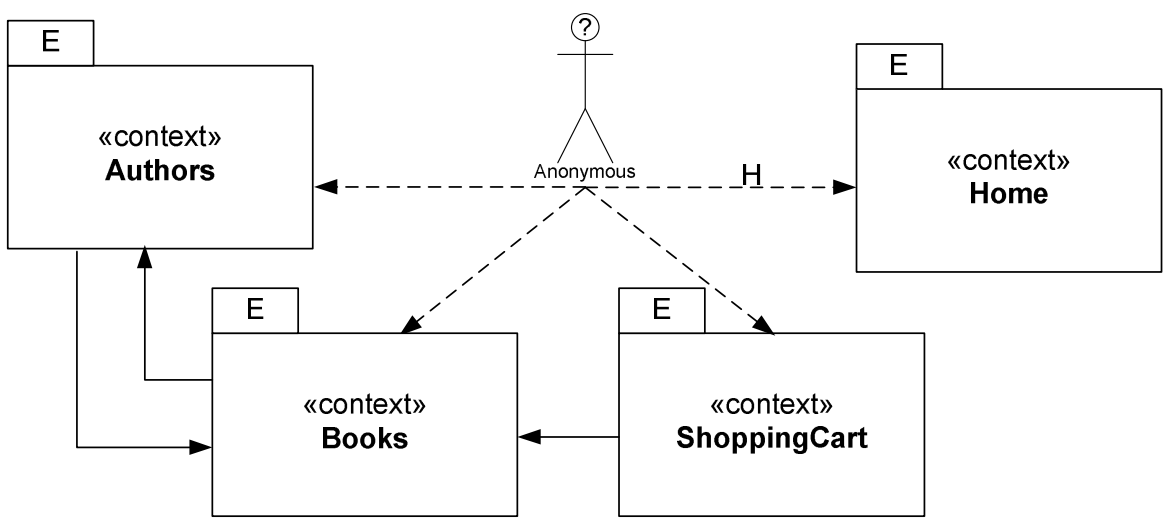

Figure 12.3 Anonymous User Navigational Map

The "Home" navigational context has been designed to show the new book purchases and also the available offers. The " $\mathrm{H}$ " label included in the navigational link indicates that the target navigational context is defined as the default context (understanding default as home content). The "ShoppingCart" navigational context shows to the user the state of her shopping cart. It displays the books included in it, as well as the number of units and the price of each book. The "Books" and "Authors" navigational contexts allow the user searching for a particular book and author respectively.

All these navigational contexts have been defined as Exploration contexts. This means that anonymous users will be able to reach any of them from any part of the web application. Moreover, the Books and Authors contexts are connected through navigational 
links (solid arrows in Figure 12.3). This connection allows propagating info that is used during the data retrieval of the target context. As a result, the target context will show information related with the received object.

\section{A.5.1.1Authoring-in-the-small}

\section{Home Navigational Context}

The "Home" navigational context (see Figure 12.4) has been defined to provide the user with information about (1) the new books that have been purchased during the last month and (2) the available book offers. This information is provided in two different AIUs as it is shown in Figure 12.4.

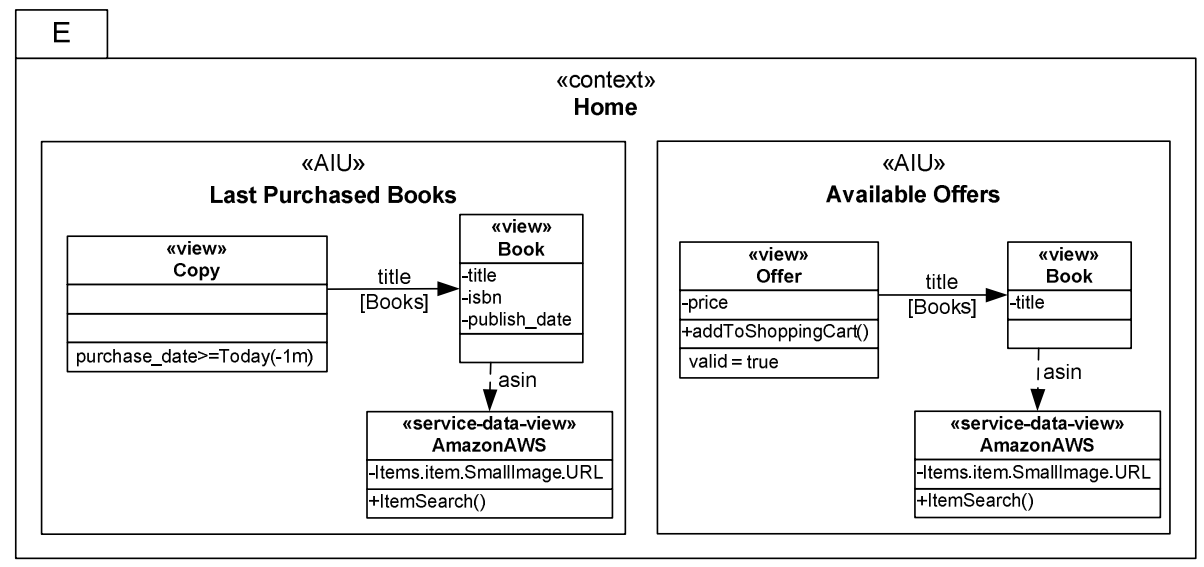

Figure 12.4 Home Navigational Context

The Home context includes views over the Structural model (class views defined over the Offer, Book and Copy classes) and the Services model (a service-data-view defined over the AmazonAWS web service). In addition, by means of the Context relationships associated to the Copy and Offer views (solid arrows) we are 
defining a navigation capability to the Books context. The Offer and the Copy class views include both a population filter. The Offer class view retrieves just those offers that are still valid. On the other hand, the Copy class view retrieves just the copies whose purchase date is later than the specified date (Today) is a local function that returns a particular date depending on the passed arguments. If no arguments are provided, the function returns the current date. On the contrary, the current date is incremented or decremented with the provided parameters).

Once the navigational context has been defined we can proceed to specify the presentation requirements. Figure 12.5 shows how the elements defined in the navigational contexts are attached with some properties regarding presentation issues. This information states the way the data is going to be organized within the graphical space according to the layout, order criteria, pagination and occurrence order patterns.

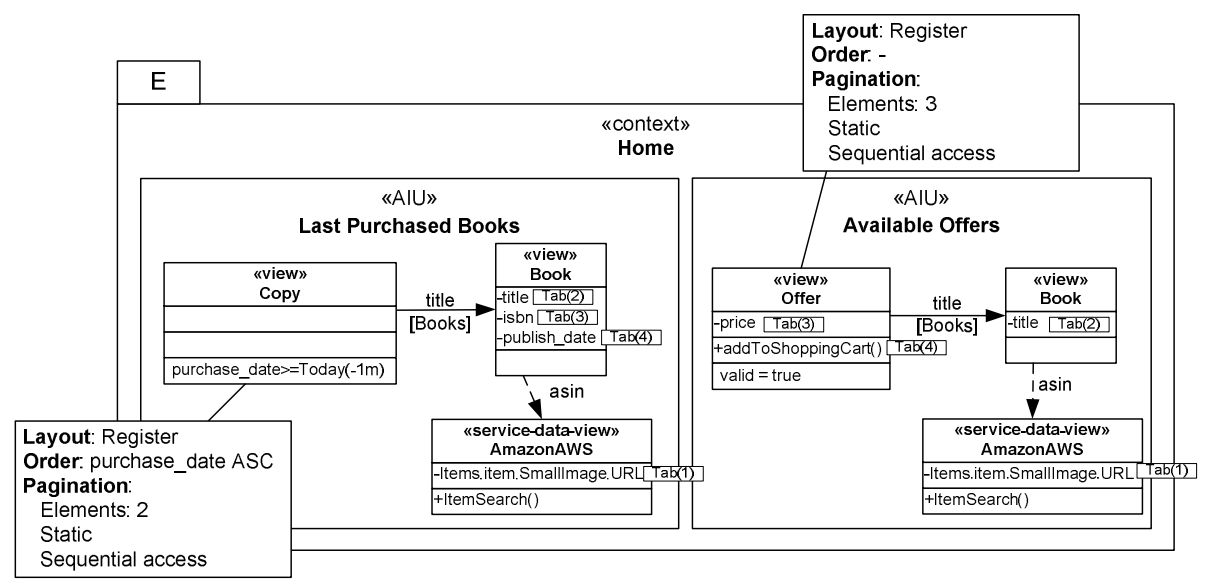

Figure 12.5 Home Navigational Context (Presentation proprieties) 
Figure 12.6 presents a snapshot of the Home Navigational Context. In it we can appreciate the navigational capability defined by exploration navigational context, the images retrieved from the Amazon web service and how the information has been organized along the graphical space according to the presentation properties associated to the context.

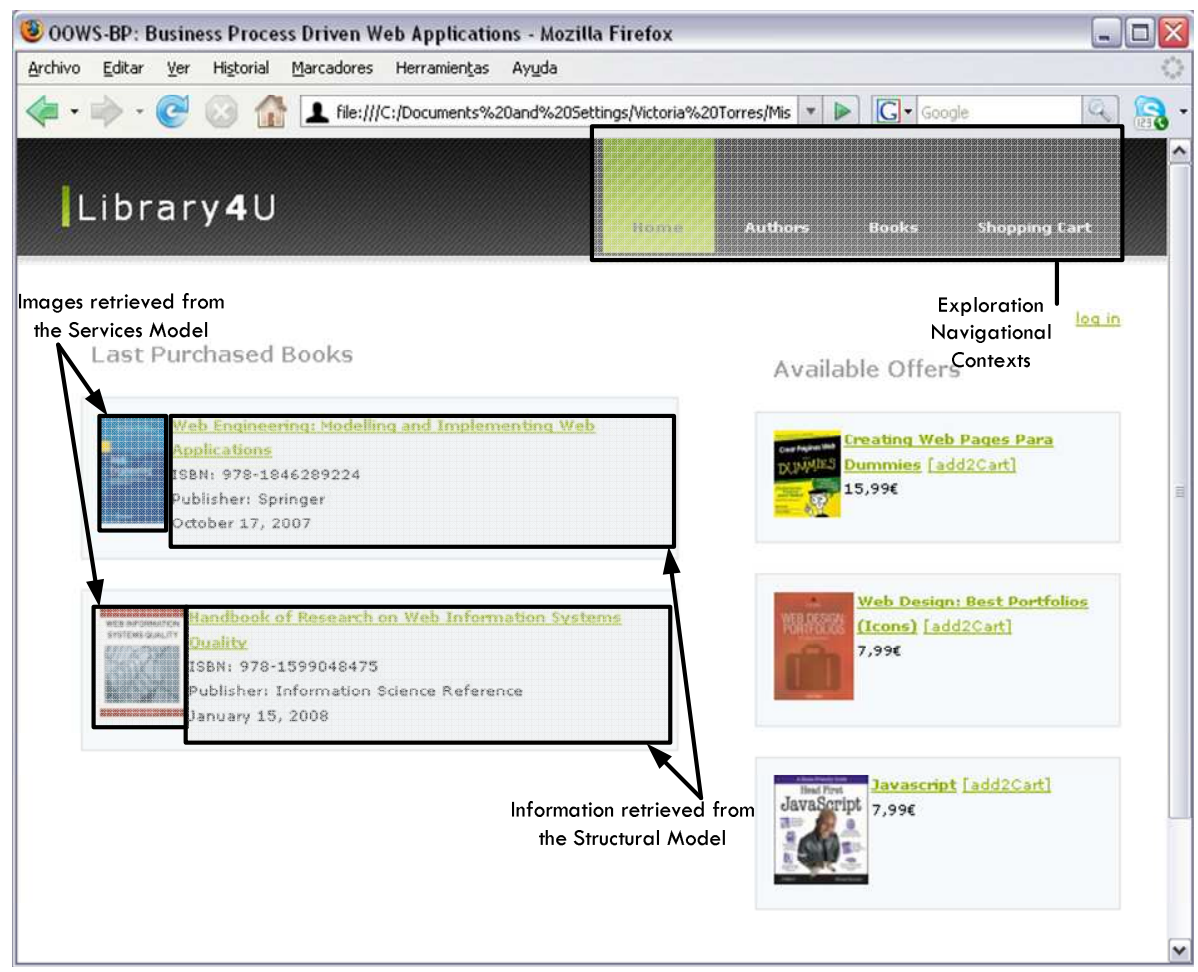

Figure 12.6 Implemented Home Navigational Context

\section{Books Navigational Context}

Similar to the Home navigational context, the Books navigational context defines views over the Structural model and the Services model. Again, the retrieved information is related with books, but 
in this case, the class view does not define any population filter (see Figure 12.7).

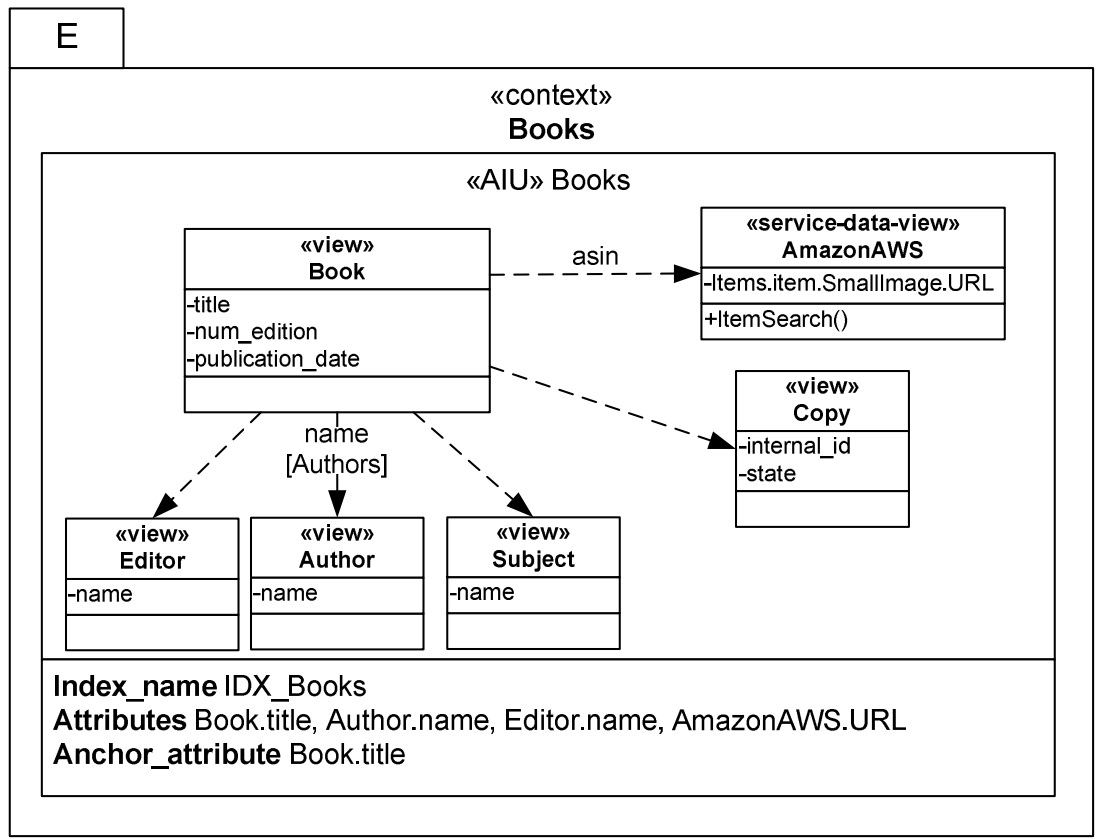

Figure 12.7 Books Navigational Context

In this case, the AIU defines an index (see bottom section of the AIU Books) which gets activated when no data is passed to the unit. This mechanism avoids overloading the web interfaces with too much data. For instance, when the Books context is achieved by means of the exploration context, the context retrieves all the book instances displaying just the information that is indicated in the index. Then, if an object of the recently obtained set is selected (by means of the anchor attribute Book.title), the context will be rendered but now with all the information defined in it. 
Related to presentation requirements, Figure 12.8 shows the presentation attributes specified to the navigational elements.

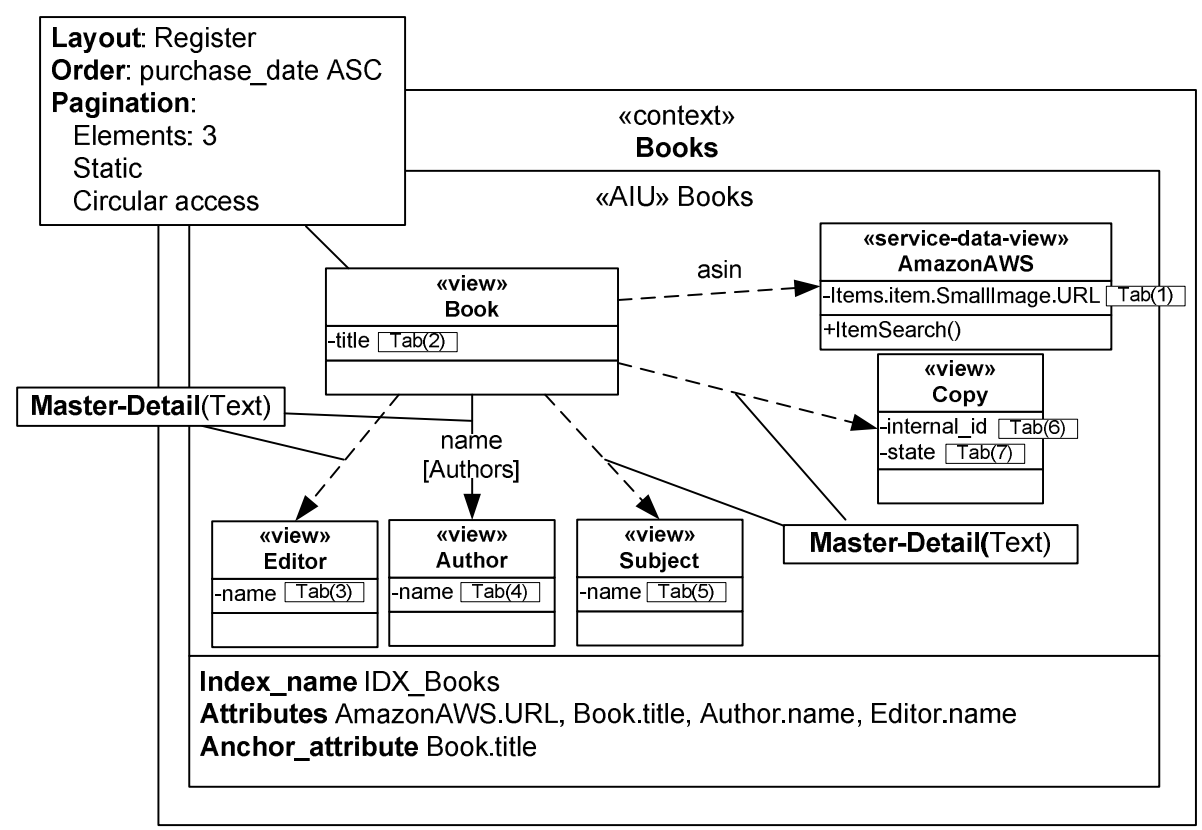

Figure 12.8 Books Navigational Context (Presentation proprieties)

Figure 12.9 and Figure 12.10 correspond to the Book context in both cases, when the index gets activated (Figure 12.9) and when the context is passed with an object reference (Figure 12.10). 


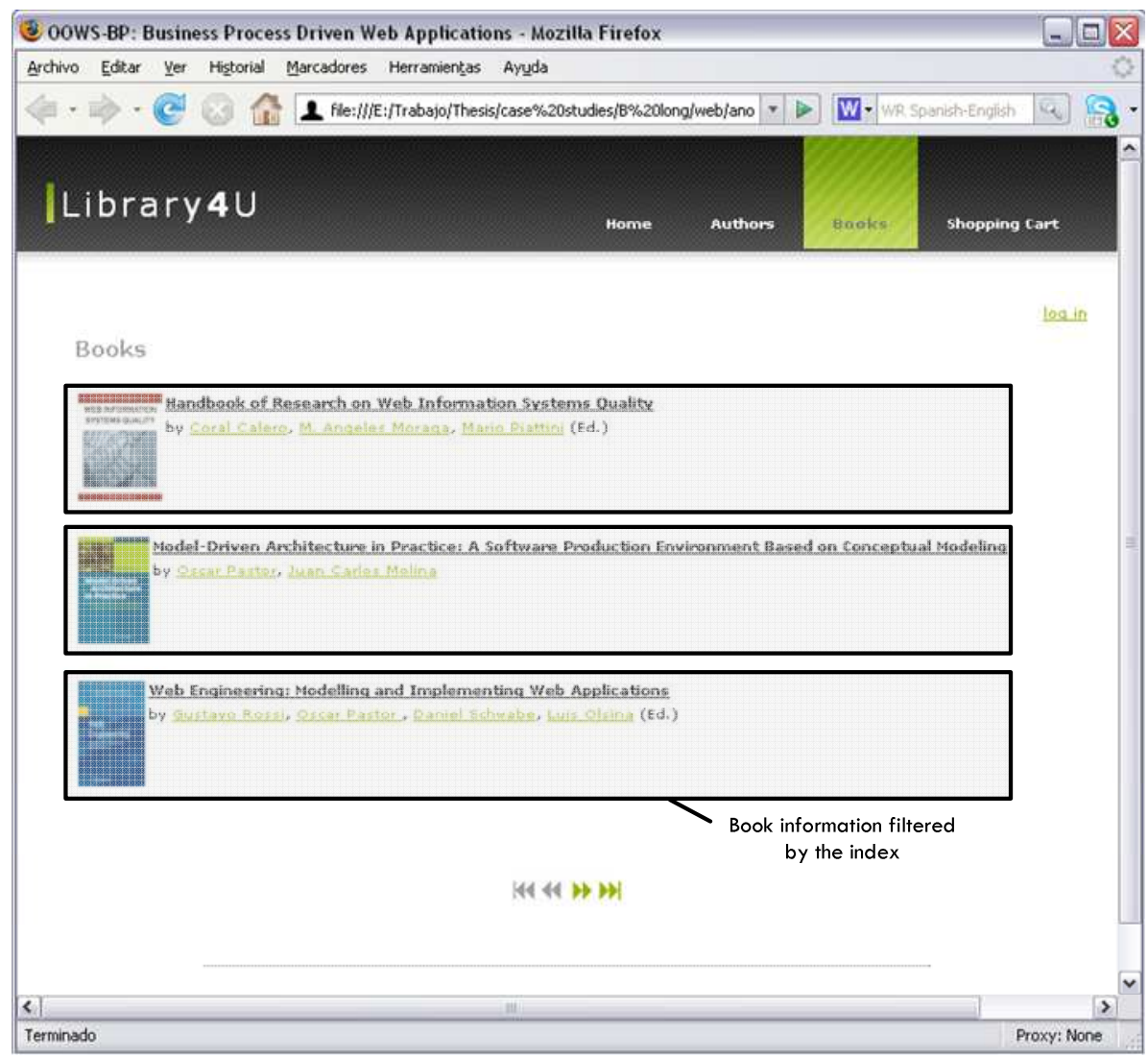

Figure 12.9 Anonymous Book Navigational Context

Each book item shown in Figure 12.9 allows navigating either to the details of the item book or to its authors (this is possible since we have associated a context relationship between the book and the author views). 


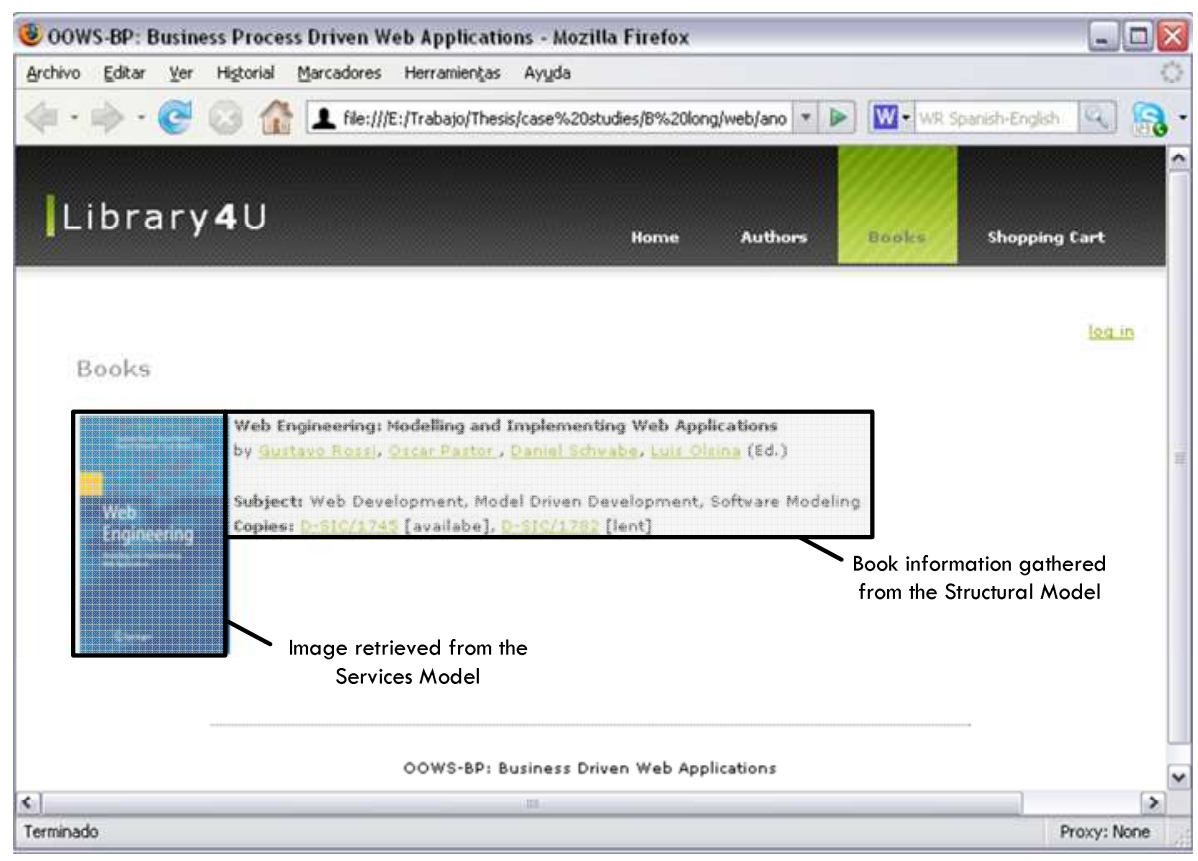

Figure 12.10 Anonymous Book Navigational Context (Detail)

\section{Authors Navigational Context}

From the Authors Navigational Context, the anonymous user can get information about authors and their related books. Figure 12.11 and Figure 12.12 shows the navigational and presentation details defined for this context. 


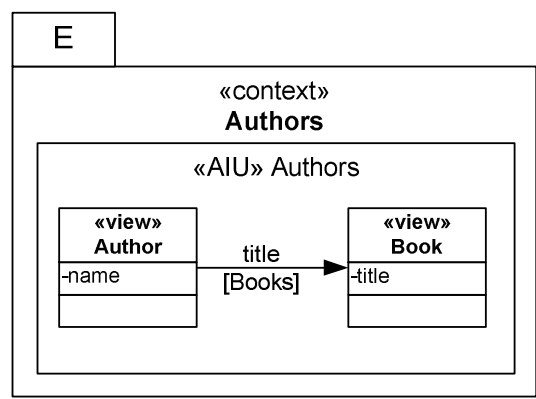

Figure 12.11 Authors Navigational Context

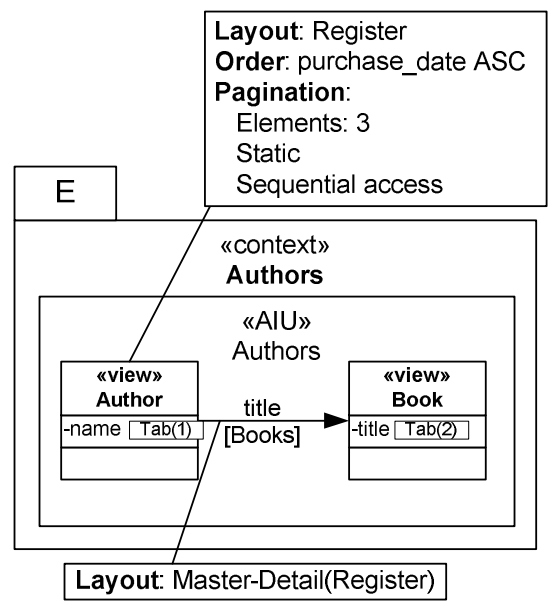

Figure 12.12 Authors Navigational Context (Presentation proprierties)

Figure 12.13 shows the implementation of the Authors Navigational Context. In this case, as we have defined only two attributes associated to the included view, it is not necessary to define an index. 


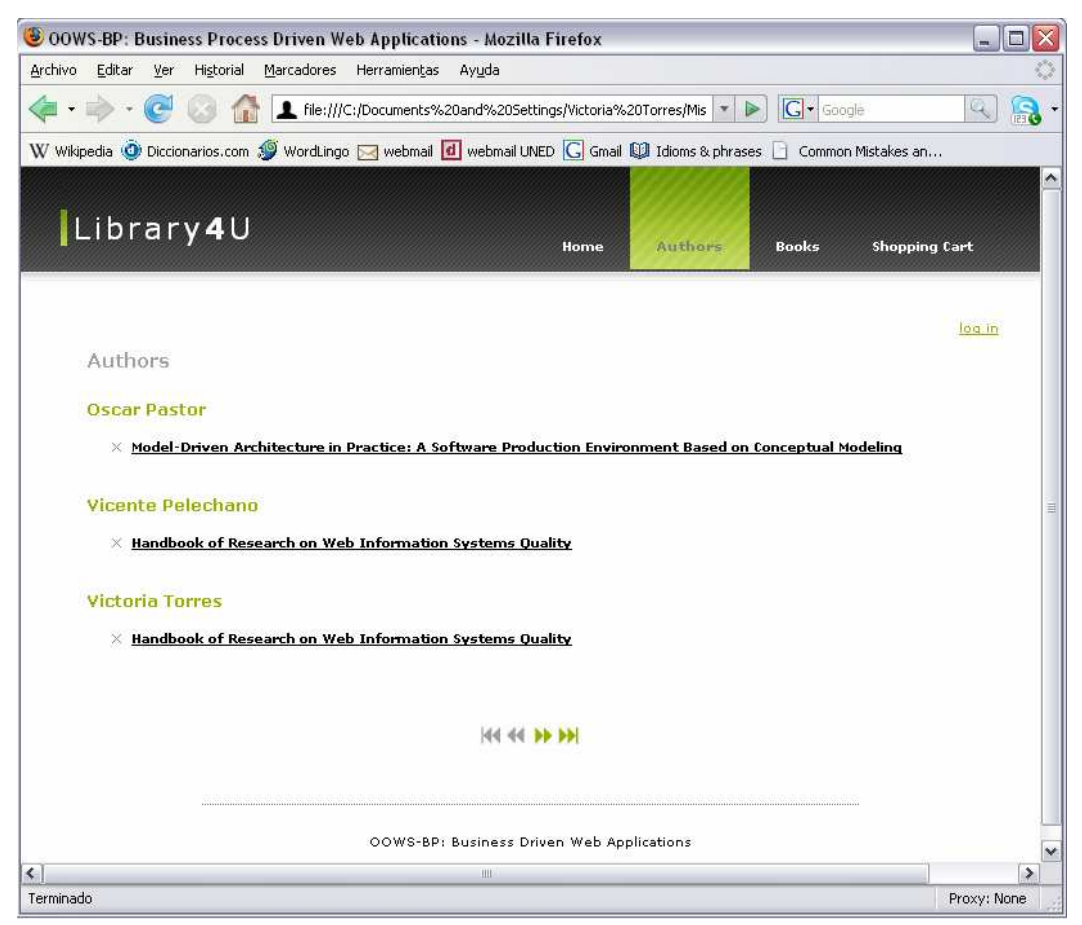

Figure 12.13 Authors Navigational Context

\section{Shopping Cart Navigational Context}

From the Shopping cart Navigational Context, the anonymous user can get information about the books added to it as well as their price and the total amount of the cart. This information is defined in the navigational context that is presented in Figure 12.14. Moreover, Figure 12.15 complements the navigational elements defined in the navigational context with the presentation properties that allow organizing the information along the graphical space. 


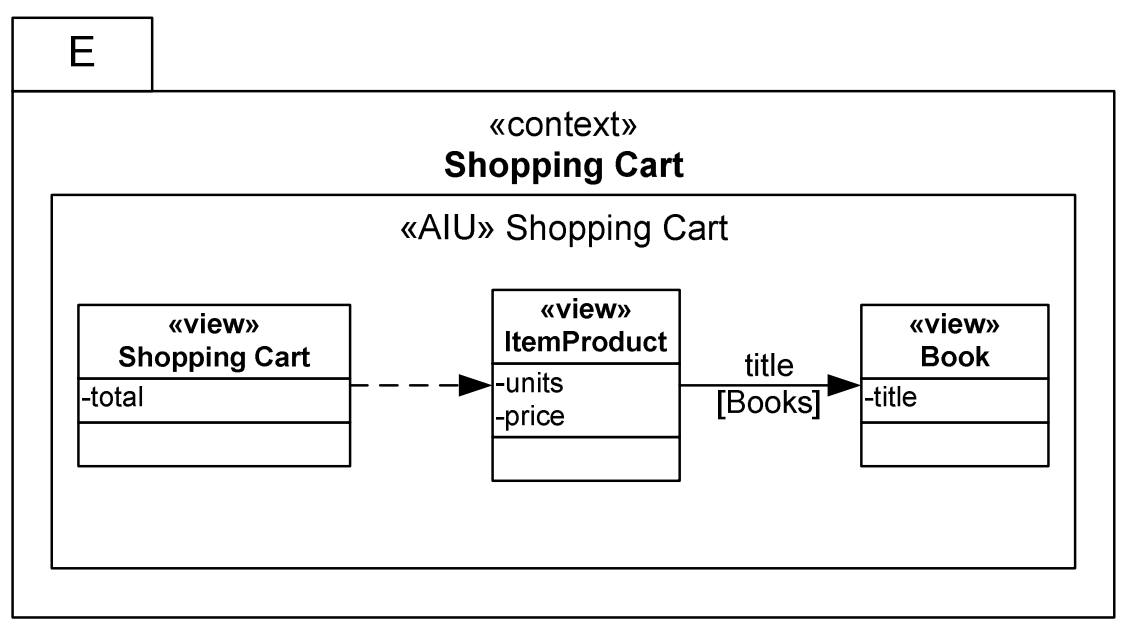

Figure 12.14 Shopping Cart Navigational Context

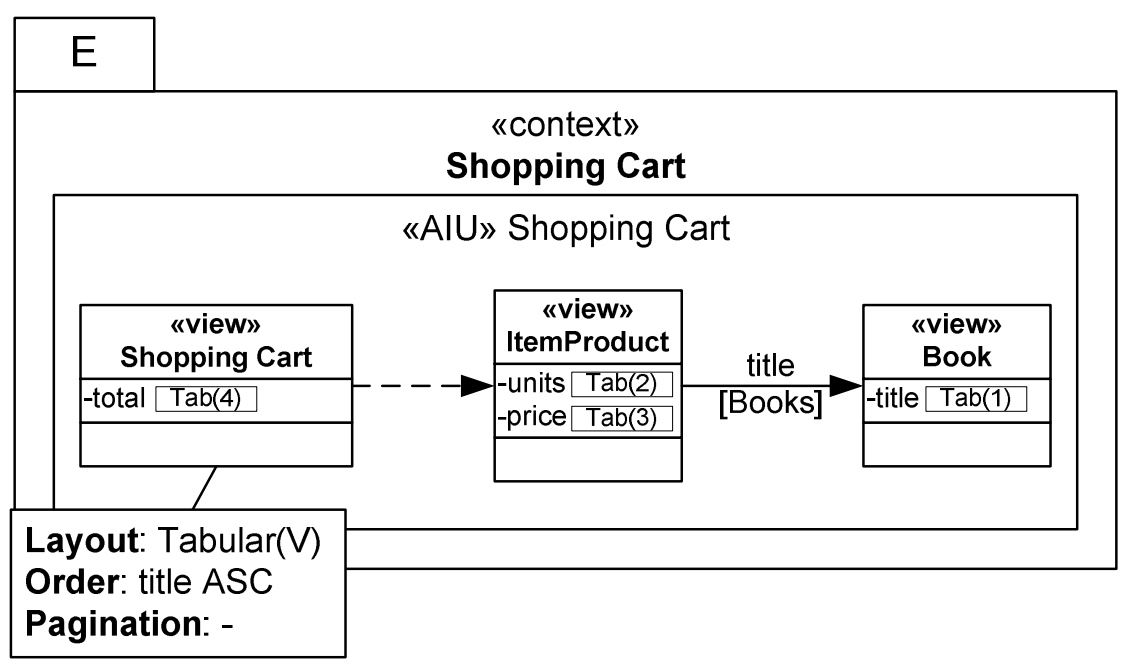

Figure 12.15 Shopping Cart Navigational Context (Presentation proprierties)

Finally, Figure 12.16 shows the Web page corresponding to the previously modelled shopping cart Navigational context. In this page we observe that the layout applied is the Tabular-Vertical. Moreover, no pagination has been defined over the retrieve 
instances. Therefore, all instances are going to be included in a unique block.

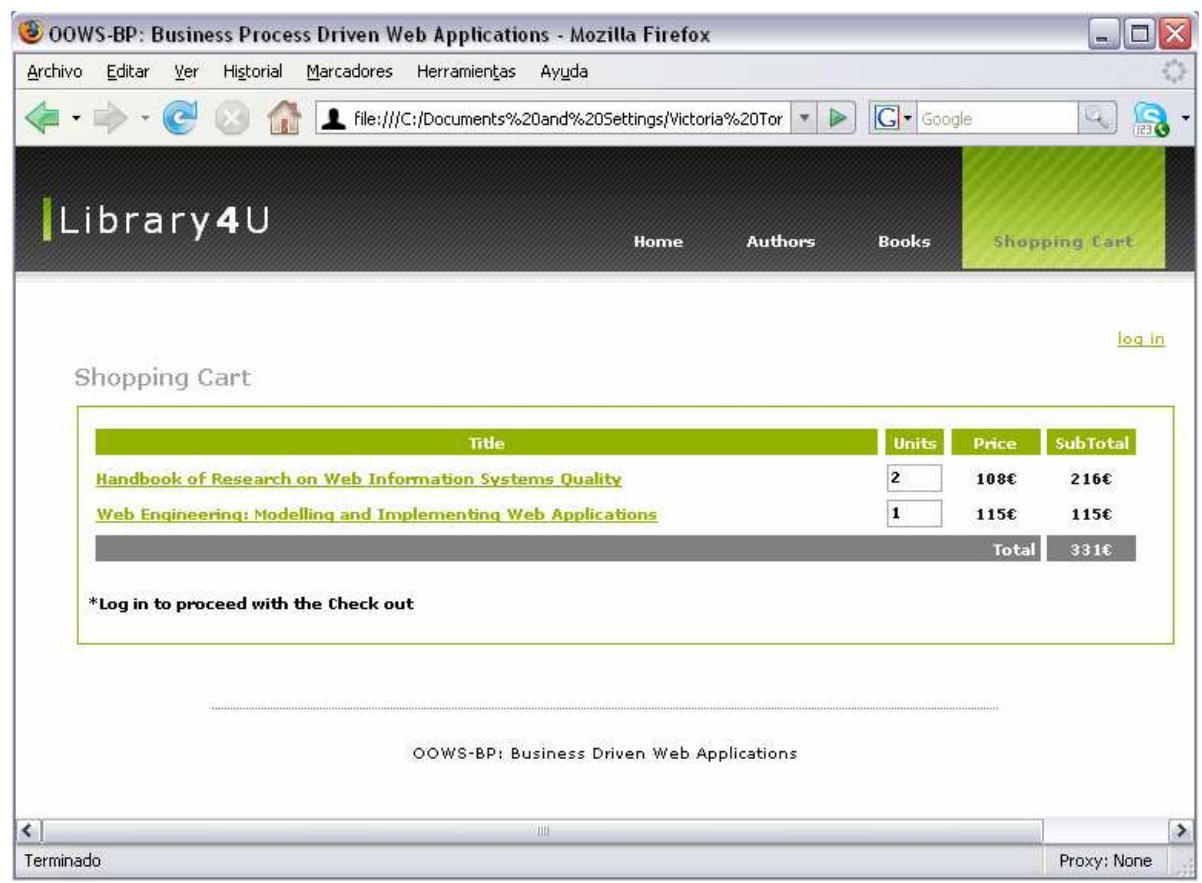

Figure 12.16 Shopping Cart Web page 


\section{Appendix B}

\section{B Short-running BP Case Study}

This appendix extends the Library $4 \mathrm{U}$ case study presented previously to give support for a short-running BP. Therefore, a description about the new requirement is presented in section B.1. Then, from section B.2 to section B.5, the Structural Model, Services Model, Business Process Model and Navigational Models are developed to deal with the modelling step of the presented short-running BP. Finally, section B.6 presents how usability issues have been considered for this kind of BP.

\section{B.1 Description}

The checkout BP is usually found in Web sites that sell products or services to their customers (sites commonly known as on-line stores). Basically, this process consist of four activities, three requiring interaction with the user (payment options, shipping address details and gift wrapping) and one that is performed completely by the system (placing the order). This BP has been classified as a short-running $\mathrm{BP}$ because satisfies all the characteristics found by this kind of BPs: it involves just one human participant (in this case this refers to the customer) who launches an instance of the BP. Moreover, the BP includes also 
collaboration with an external system which charges the shopping to the customer account.

\section{B.2 Structural Model}

The part of the Structural model related to the Checkout BP is the one presented in Figure 12.1. This model just includes the operations that are invoked by the BPEL process in order to perform a specific task.

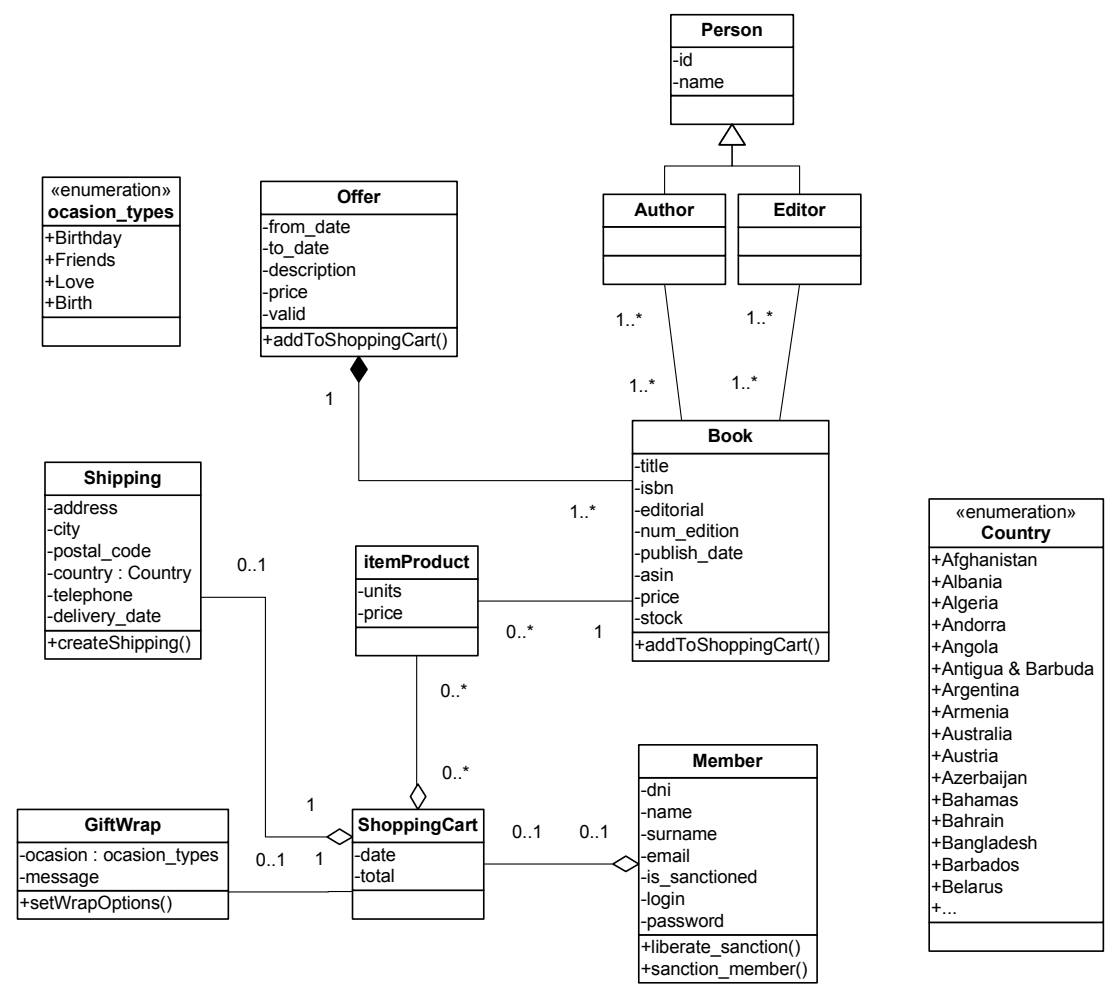

Figure B.1 Structural Model

\section{B.3 Services Model}

The set of services that are going to be consumed in the current $\mathrm{BP}$ involves just one partner which represents a service providing 
support to perform payments via Internet. The service provided by this partner is used in the process to charge the user account with the amount spent during the shopping.

The external functionality that is required in the Checkout BP is provided by an on-line Payment Service. This functionality is provided as a Web service in the corresponding WSDL file. Code excerpt B.1 shows the port type section of the WSDL document which defines the set of operations performed by the Web service.

<wsdl:portType name="onLinePaymentService" > $<$ wsdl:operation name=" DoDirectPayment ">

$<$ wsdl:input message=" ns:DoDirectPaymentRequest " $></$ wsdl:input $>$

$<$ wsdl:output message=" ns: DoDirectPaymentResponse" $></$ wsdl:output $>$ $</$ wsdl:operation $>$

$</$ wsdl:portType $>$

Code excerpt B.1 WSDL PortType definition

Through the "DoDirectPaymentRequest" operation we can charge the user with the amount of the order. This operation requires data such as the credit card details and the amount to charge (see Code excerpt B.2).

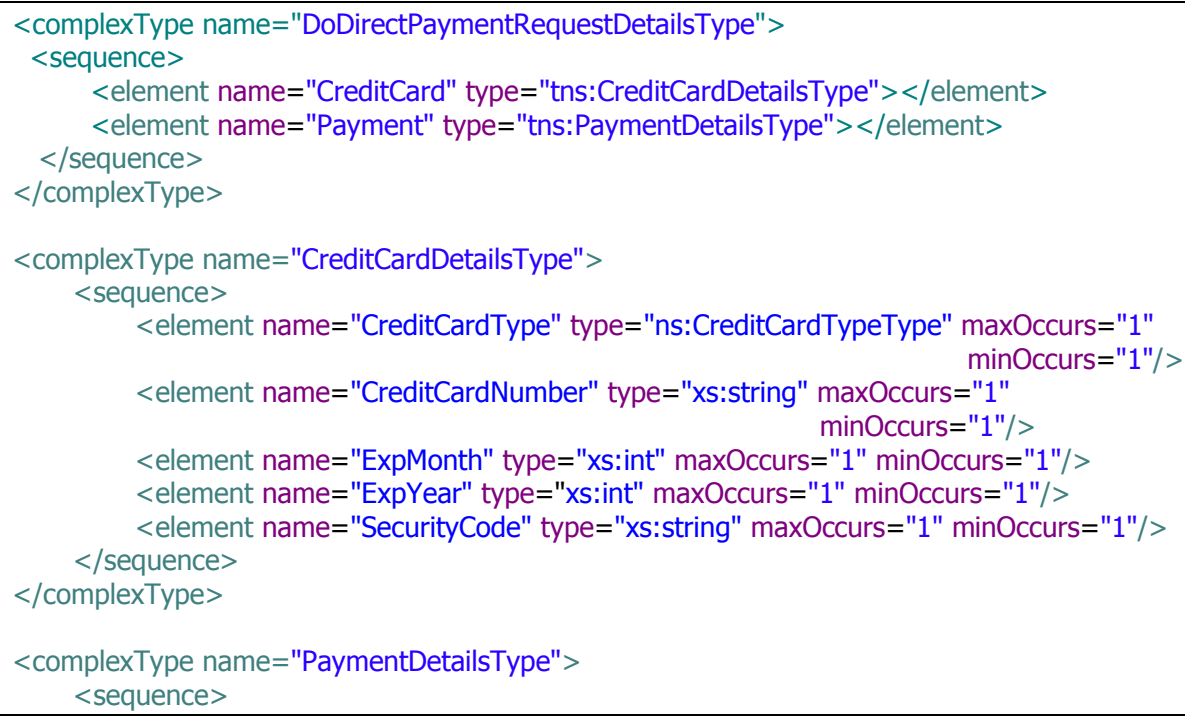


<element name="OrderTotal" type="ns:string" maxOccurs="1" minOccurs="1"/>

<element name="ItemTotal" type="xs:string" maxOccurs="1" minOccurs="1"/>

<element name="ShippingTotal" type="xs:string" maxOccurs="1" minOccurs="1"/>

$</$ sequence $>$

$</$ complexType $>$

Code excerpt B.2 Data types used by the "DoDirectPayment" operation

\section{B.4 Business Process Model}

The BP defined in this case is made up of two entities, one referring to the Library $4 \mathrm{U}$ Company (the internal system) and another referring to the payment service (provided by an external system).
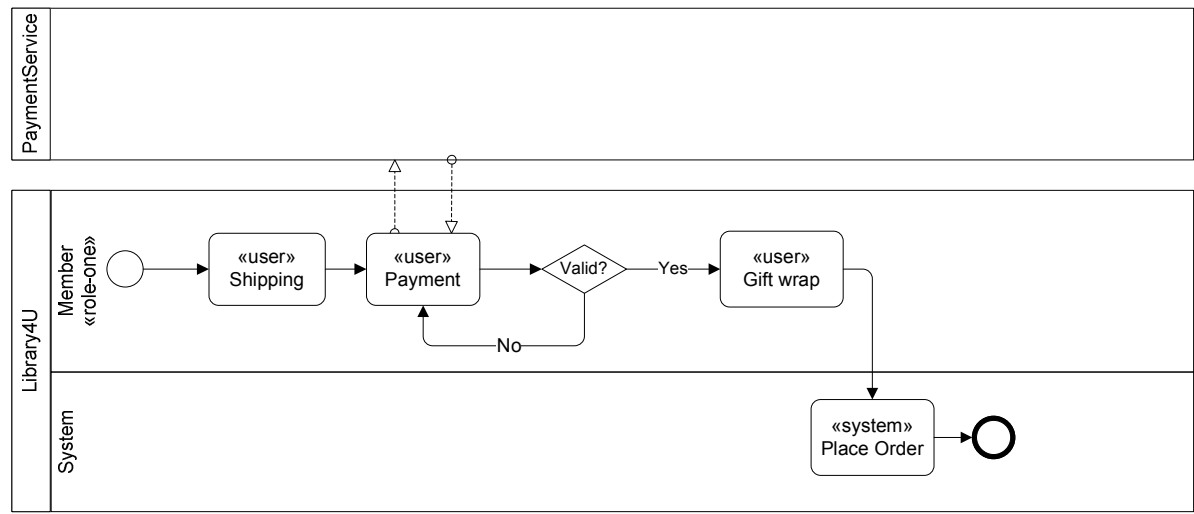

Figure B.2 Business Process Model

Moreover, within the internal system we distinguish two roles, the human participant (being represented by the member) and the system, referring this latter to the functionality that is provided by the internal system and that do not requires interaction with the user.

\section{B.5 Navigational Model}

This section presents the navigational model defined for the member user. 


\section{B.5.1 Authoring-in-the-large}

The only navigational capability that we want to add to the Navigational Model inherited from the anonymous user is the possibility of proceeding with the check out of the shopping cart. For this reason, as Figure B.3 shows, we have added just one Process Context which represents the entry point to the process.

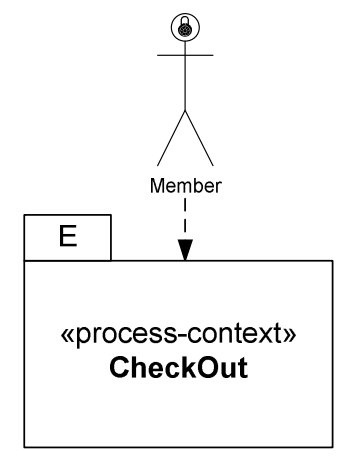

Figure B.3 Registered User Navigational Map

This Process Context has been defined as Exploration Context in order to make it accessible to the user from any part of the application. The details of this context are presented in the following subsection.

\section{B.5.2 Authoring-in-the-small}

The Checkout Process Context, as Figure B.4 shows, includes three Activity Containers (one per each activity requiring interaction with the user, see Member lane within the Library4U pool in Figure B.2). 


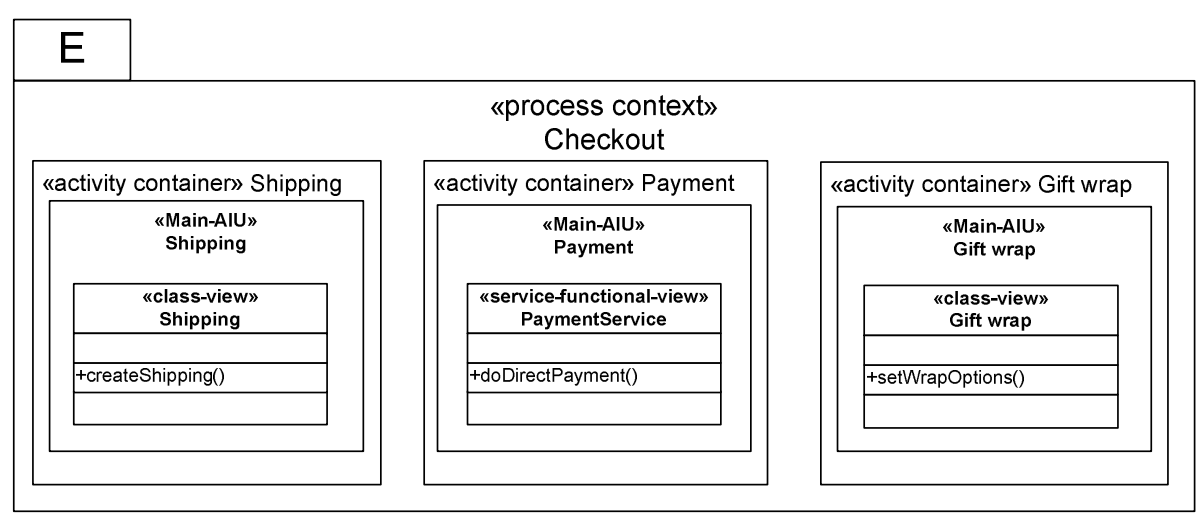

Figure B.4 Checkout Navigational Context

From the set of Activity Containers included in the Checkout process context, two include views over classes defined in the Structural model (the Shipping and Gift wrap activity containers) and one defines a view over a service from the Services model (the Payment activity container). In this case, all the containers are used to gather data from the user and no additional information is shown to the user. Figure B.5, Figure B.6 and Figure B.7 show respectively the Web pages equivalent to the activity containers included in the Checkout Process Context.

In these figures we have stressed four areas which correspond to (A) the set of tasks that has to be completed by the user in order to complete the process, (B) the entry point to the process (the context was defined as exploration), (C) the label indicating the task being performed at each moment and (D) the area provided to the user to introduce the information required to accomplish the current activity. 


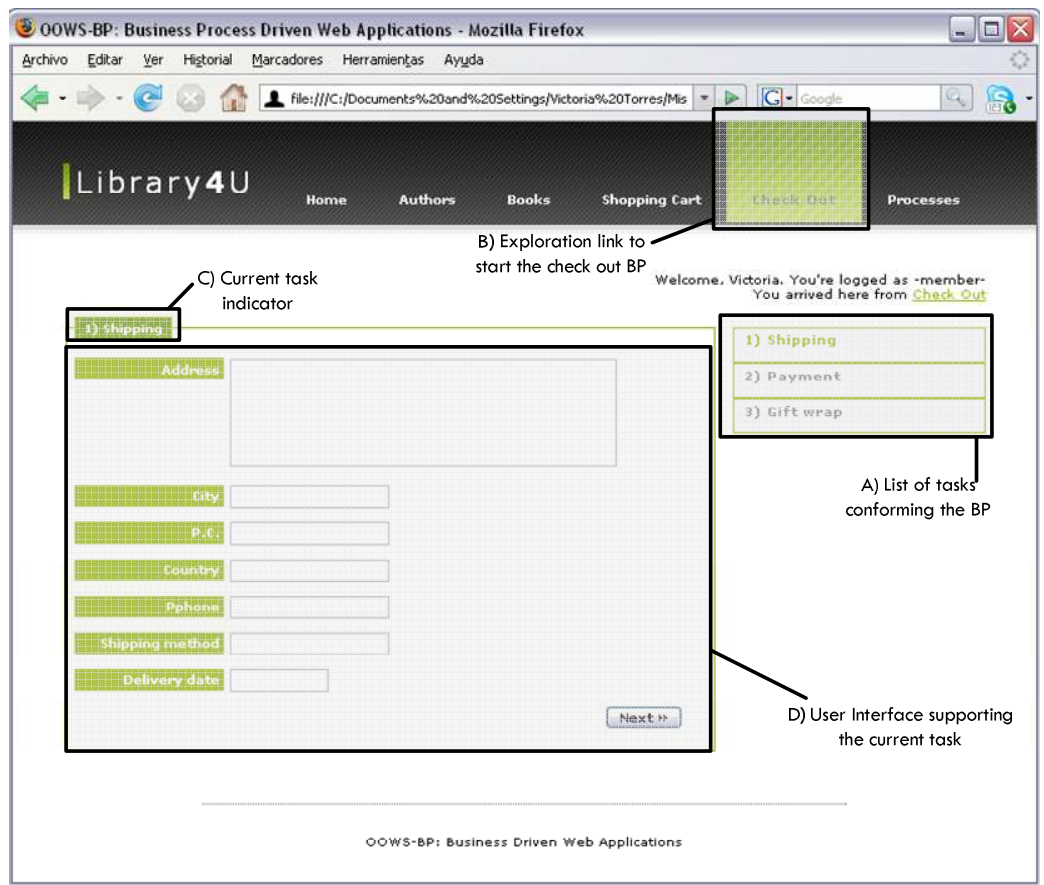

Figure B.5 Generated User Interface for the "Shipping" task

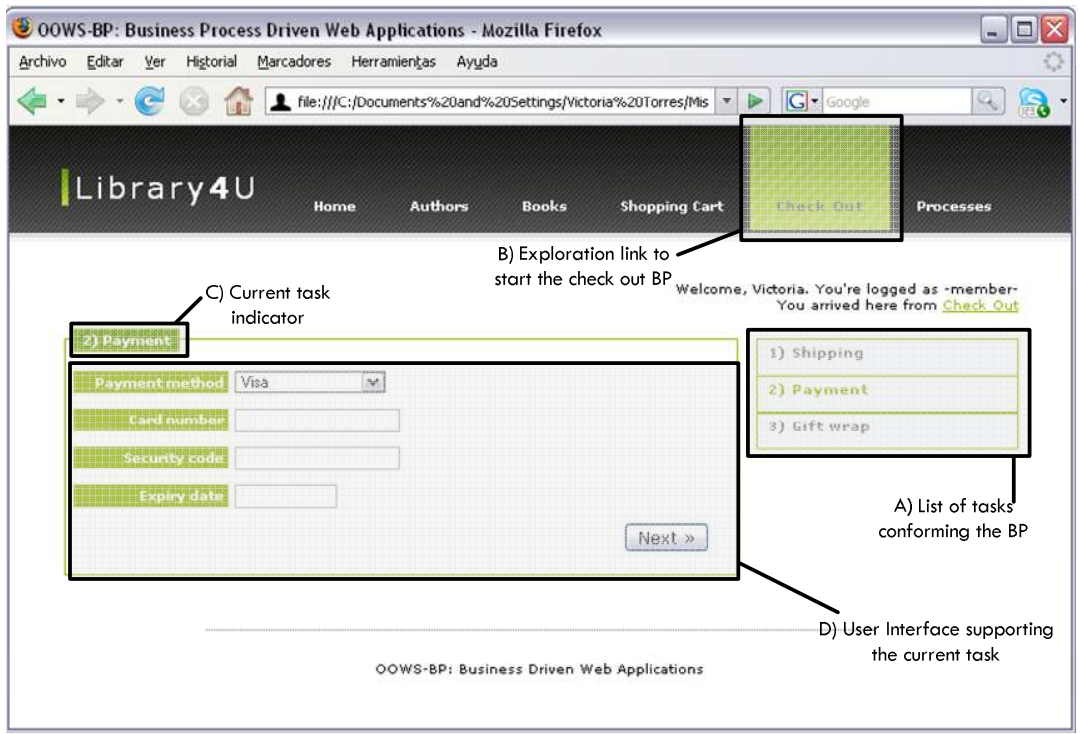

Figure B.6 Generated User Interface for the "Payment" task 


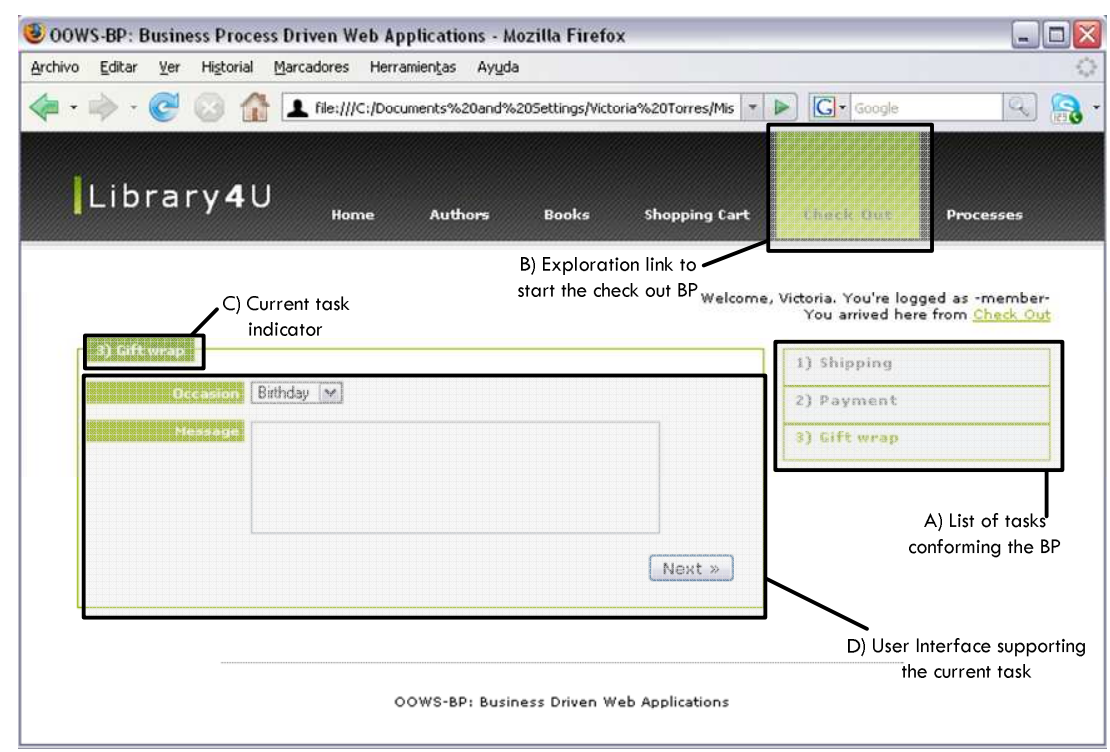

Figure B.7 Generated User Interface for the "Gift wrap" task

\section{B.6 Usability Aspects}

The application of a set of usability guidelines during the transformation process ensures the production of usable web applications. For instance, in this case, as the current case study deals with a short-running process, the generated user interfaces include the wizard pattern. This pattern can be seen in Figure B.5, Figure B.6 and Figure B.7 in the remarked area labelled with an "A" and allow displaying the user the set of tasks that made up the process. Moreover, an indicator of the current task is also included in the interface (see the remarked area labelled with a "C" and also the colour of the corresponding task in the wizard in $\begin{array}{lllll}\text { Figure } & \text { B.5, Figure } & \text { B.6 and } 6 \text { Figure }\end{array}$ 


\section{Appendix C}

\section{Long-running BP Case Study}

This appendix extends the case study presented in appendix A with a long-running process. In particular the extension deals with the Book Request Purchase BP (hereafter BRP-BP) which is presented in detail in the first subsection. The remainder of the appendix is structured as follows. Section C.2 rescues those parts from the structural model presented in appendix $\mathrm{A}$ that are related with the applicable BP. Section C.3 extends the Services Model with the service and operations related to the BPR-BP. Then, section C.4 presents the Business Process Diagram representing the BRP-BP. Finally, section C.5 develops the Navigational and Presentation models for the registered users modelled in the User Model in Appendix A.

\section{C.1 Description}

The BRP-BP is a kind of process quite common in any organization. This kind of processes basically consists in three steps which are (1) someone applying for something (i.e. a certificate, a service, a good, etc.) (2) someone validating the application and (3) in case the validation was positive, provide the 
applicant with the requested thing. In this process, during the last two steps more than one person or service can be involved.

In particular, the present case study deals with the purchase and later loan of a book that is not in the library. The request is launched by a member of the library, validated by the secretary staff of the library based on a particular criterion, purchased by the central library (an external system), and finally loan by the librarian staff.

This BP has been classified as a long-running BP because satisfy all the characteristics found by this kind of BPs. Multiple human participants, corresponding to different roles within the organization, are involved in the BP. Moreover, the BP includes also collaboration with an external system which performs the book purchase.

\section{C.2 Structural Model}

The excerpt of the structural model presented in this section includes all the classes that are related to the RBP-BP. These classes define the operations that are going to be associated to the activities that make up the process. 


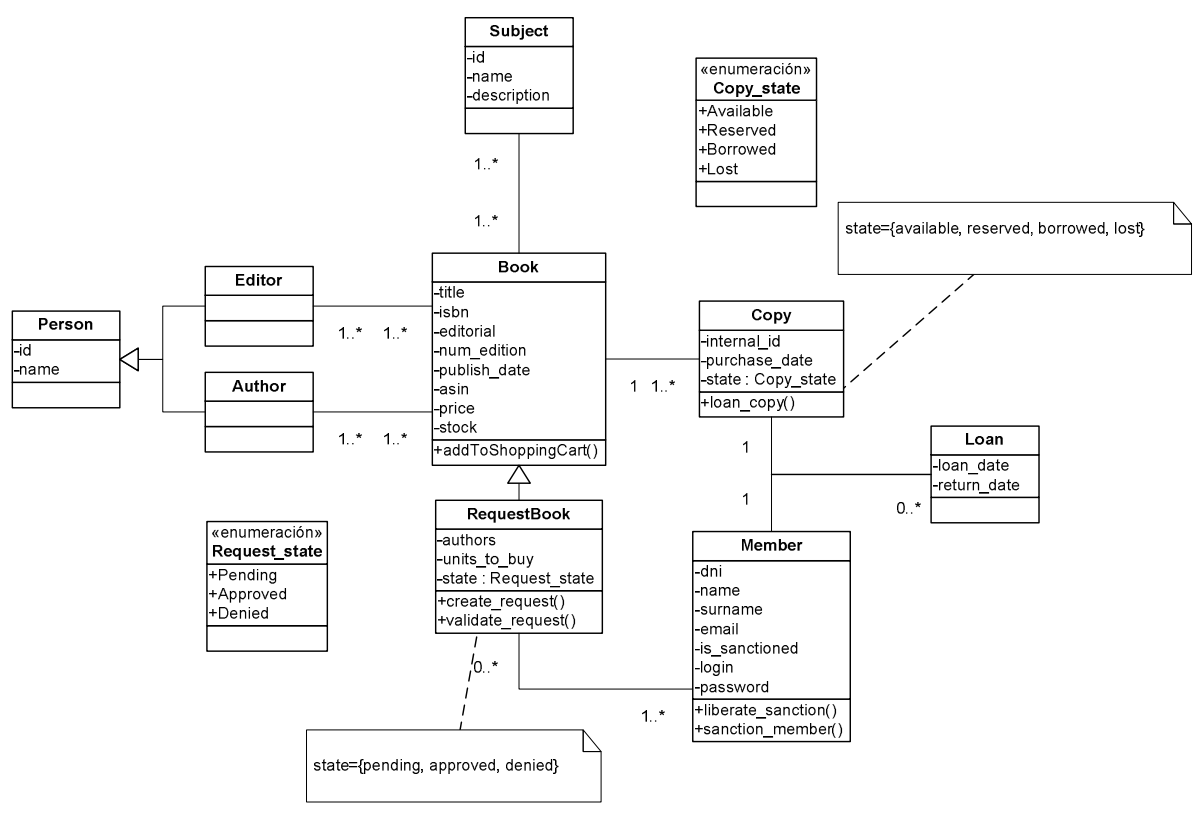

Figure C.1 Excerpt of the Structural Model

\section{C.3 Services Model}

The external functionality that is required in the BRP-BP is provided by the central library of the university. This functionality is provided as a Web service in the corresponding WSDL file. Code excerpt B.1 shows the port type section of the WSDL document which defines the set of operations performed by the Web service.

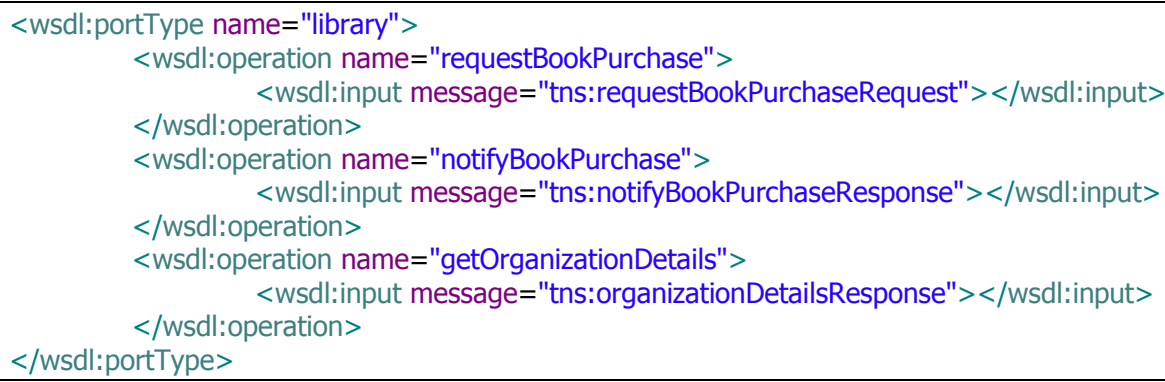

Code excerpt C.1 WSDL PortType definition 
The "requestBookPurchase" operation allows us notifying the central library about the necessity of buying a particular book. This operation requires some data about the book requested such as its "isbn" or "title" (see Code excerpt B.2).

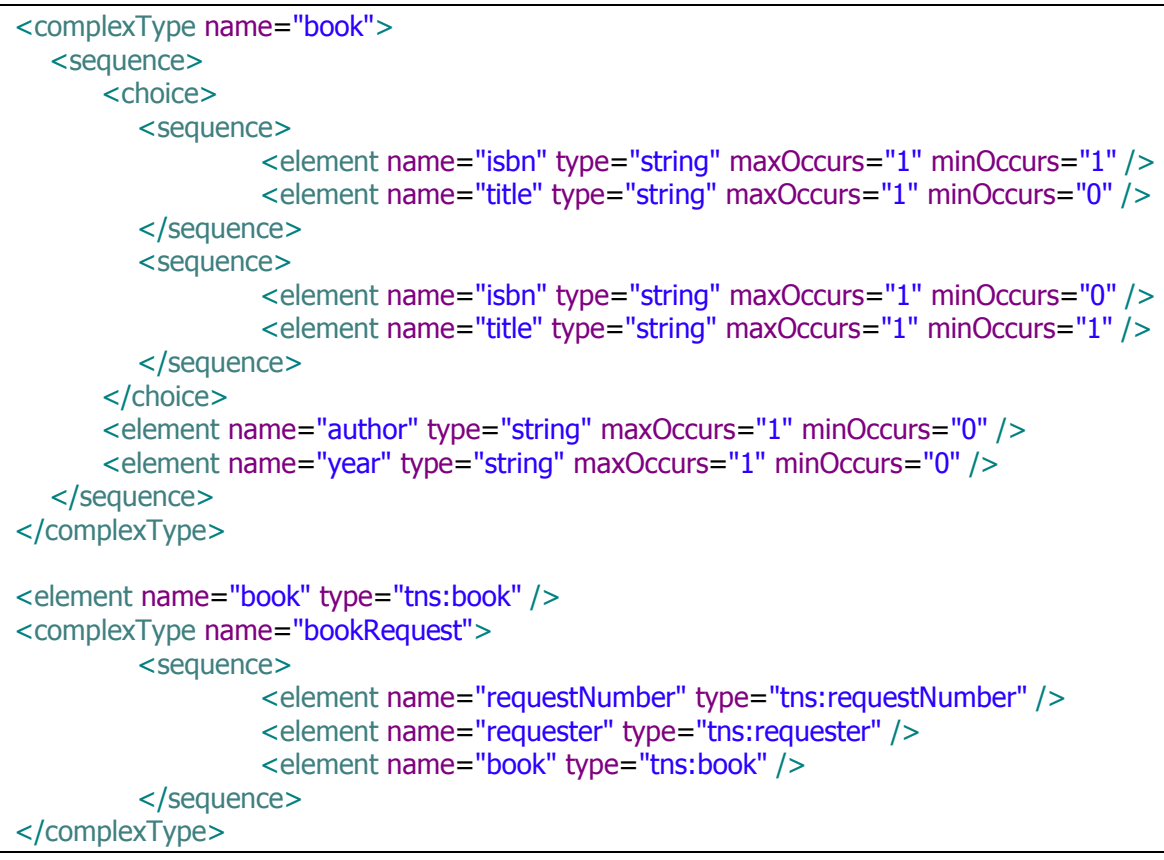

Code excerpt C.2 Message type required by "requestBookPurchase" operation

\section{C.4 Business Process Model}

The Business Process Model allows defining the set of activities that have to be performed in order to buy and loan a particular book. Moreover, it allows distributing these activities among the involved participants, being these humans or systems. 


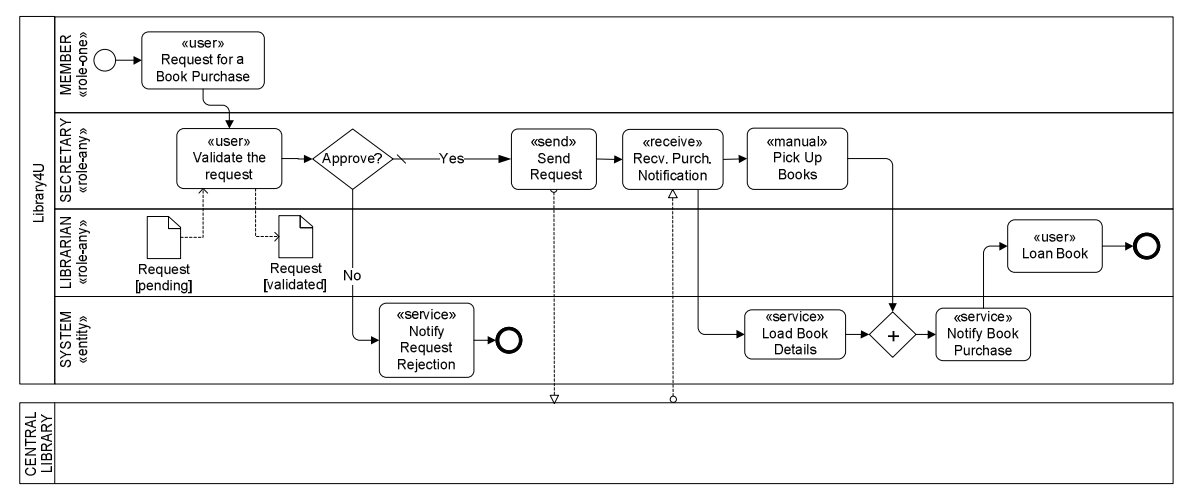

Figure C.2 Business Process Model

In this particular case, the $\mathrm{BP}$ is made up of two entities (represented graphically as pools), one referring to the Lybrary4U system (the internal system) and another referring to the central library (the external system). Moreover, within the internal system we distinguish a set of human participants (which correspond to different roles within the organization) and a nonhuman participant, the system, which refers to the functionality that is provided by the internal system. Each of the activities included in the business process diagram has been associated to operations defined either in the Structural or the Services models. For instance, the first activity of the BP (the "Request for a Book Purchase") has been associated to the create_request() operation defined in the RequestBook class. This operation creates an instance of this class registering a new book purchase application. In addition, this activity creates an instance of the BRP-BP.

\section{C.5 Navigational and Presentation Models}

This section develops the Navigational and Presentation models for all the users involved in the process. These users, by means of 
the inheritance relationship, extend the navigational map defined for the anonymous user presented in the appendix A. Again, the following subsections are going to be organized in two parts attending to the two steps in which the Navigational model is built (Authoring-in-the-large and Authoring-in-the-small).

\section{C.5.1 Member Navigational and Presentation Model}

\section{C.5.1.1Authoring-in-the-large}

Since the member user is involved in the BRP-BP, a new context is added into her Navigational map. However, in this case the context refers to a process context. This new context extends the navigational map inherited from the anonymous user. Figure C.3 depicts the Navigational Map for the member user. When the inherited contexts are not modified it is not necessary to include them in the map again.

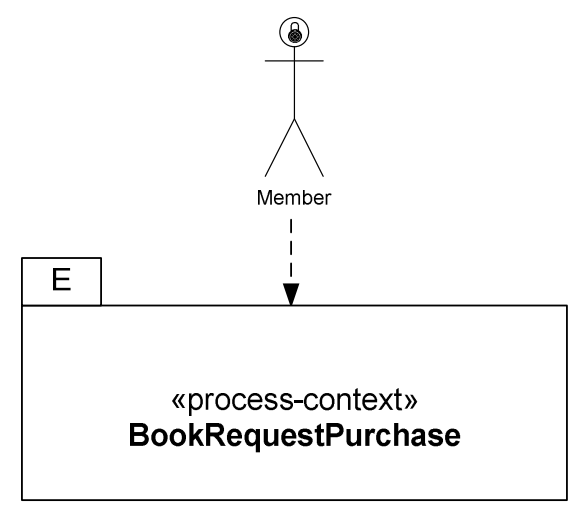

Figure C.3 Member Navigational Map

This process context has been defined as Exploration Context (context labelled with an "E" in Figure C.3) in order to make it 
accessible from any part of the application (see "Processes" link in Figure C.4).

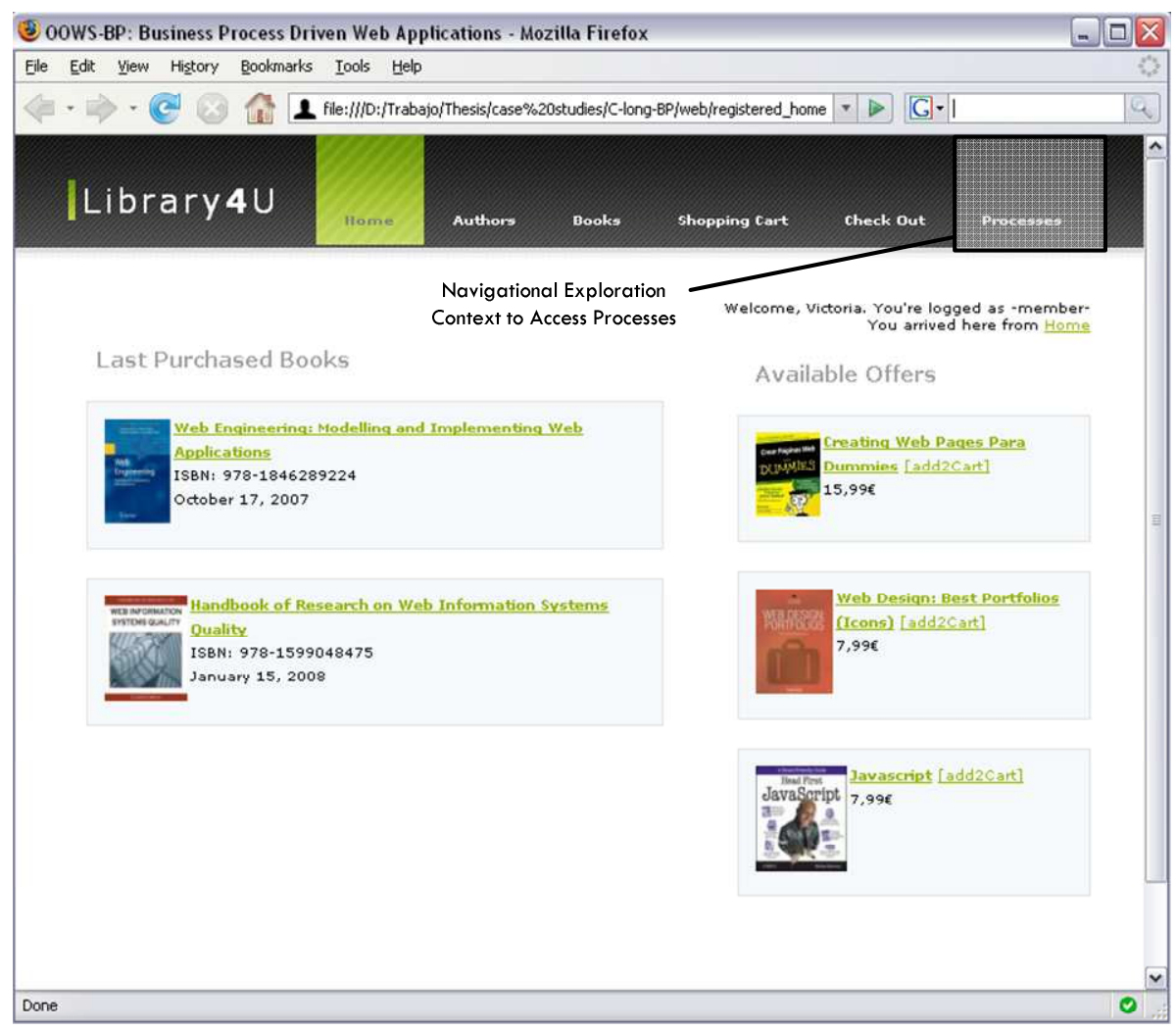

Figure C.4 Home page including the Processes Link

The "Processes" link included at the top of the Web page (Figure C.4) constitutes the entry point to the Web application that supports the execution of long-running processes. Figure C.5 shows the Web page displayed to the user after pressing over the "Processes" link. In this page, the list of pending tasks associated to the connected user is shown. On the one right hand side of the page, the stressed navigation area allows the user (1) accessing to her list of pending tasks and (2) launching instances of all those 
processes in which this particular user is involved. On the left hand side of the page, the user is displayed with her current pending tasks. This latter area allows the user completing the tasks that are active at any moment.

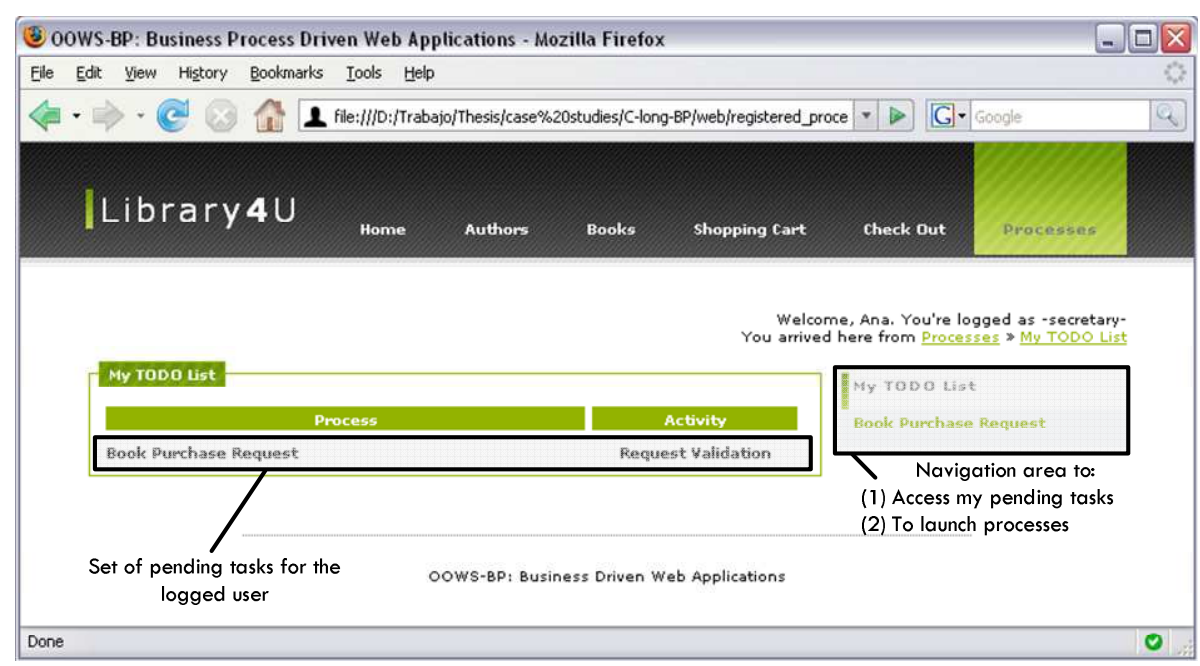

Figure C.5 My TODO List for a Secretary user

Once the global view of the map has been defined, it is necessary to specify the details of the new context.

\section{C.5.1.2Authoring-in-the-small}

The Request Book Purchase Process Context, as Figure B.4 shows, includes just one activity container which represents the interaction required with the user to launch an instance of the process. 


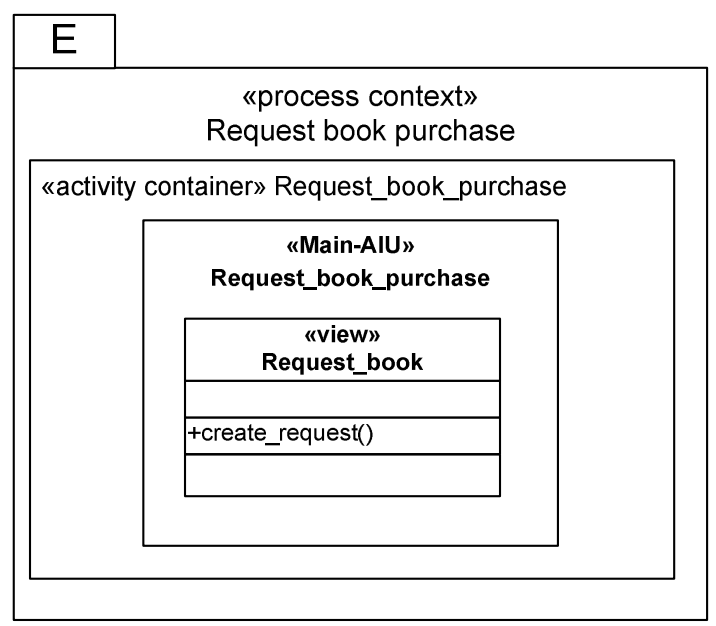

Figure C.6 RBP Process Context for the Member user

The equivalent web page to the process context is shown in Figure C.7. In this case, the left hand side of the page has been substituted by the necessary interaction mechanisms that allow the user providing the required data to complete the current activity.

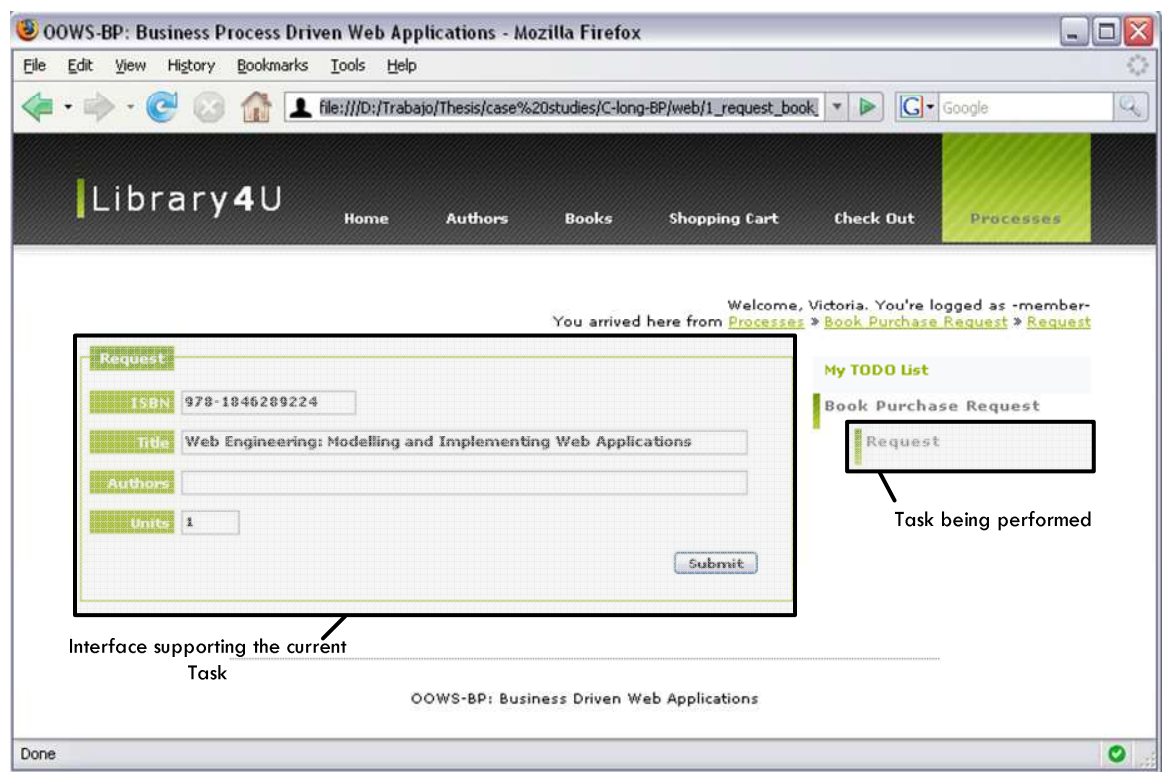

Figure C.7 Request Book Purchase task for the member user 
In this case, since the task simply involves the introduction of some data from the user, it is not necessary the application of any presentation pattern. For this reason the definition of presentation requirements is skipped for this process context.

\section{C.5.2 Secretary Navigational Model}

\section{C.5.2.1Authoring-in-the-large}

Similarly to the member user, the navigational map of the secretary user includes a process context associated to the BRPBP. However, since we defined a inheritance relationship between the member and the secretary user types, in this case the process context is going to be redefined from the one inherited from the member user (see Figure C.8).

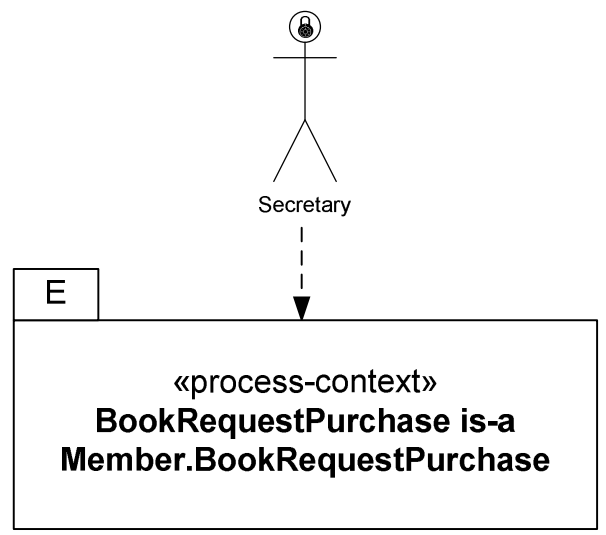

Figure C.8 Secretary User Navigational Map

The redefinition of this context is presented in the following subsection. 


\section{C.5.2.2Authoring-in-the-small}

In this case, the secretary user type participates in two of the activities included in the BPR-BP, which correspond to the "Validate the request" and the "pick up books" user and manual tasks. For this reason, her associated process context includes two activity containers, one per each interaction defined in the BPM with the secretary user (see Figure C.10). On the one hand, the Validate_request activity container allows the secretary user to validate the requests that are pending to solve. This is specified by means of the population filter applied to the Request_book view. In this case only request book instances whose state attribute is set to the values "pending" or "rejected" are going to be retrieved. Figure C.9 shows the basic process context necessary to complete the tasks committed to the secretary role.

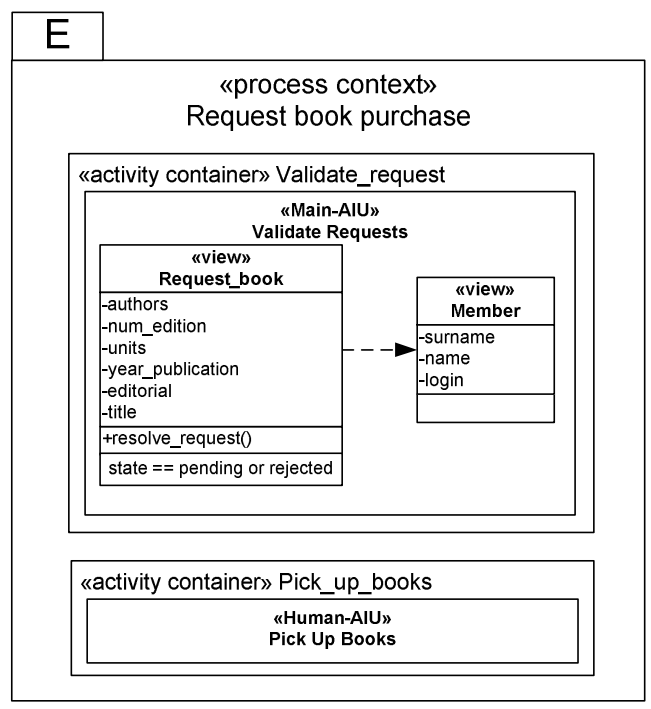

Figure C.9 Details of the Request Book Purchase Process Context However, activity containers can be enriched by adding new constructs that help users in completing tasks (see Figure C.10). 
In particular, the Validate_request activity container has been enriched with two complementary AIUs. These AIUs add some useful information to help the user in the decision of validating or rejecting the requests. In this case the Last Purchases Complementary AIU shows the user the book copies that have been purchased in the last month. On the other hand, the Sanctioned Members Complementary-AIU shows the department members that are sanctioned and that cannot apply for any book purchase.

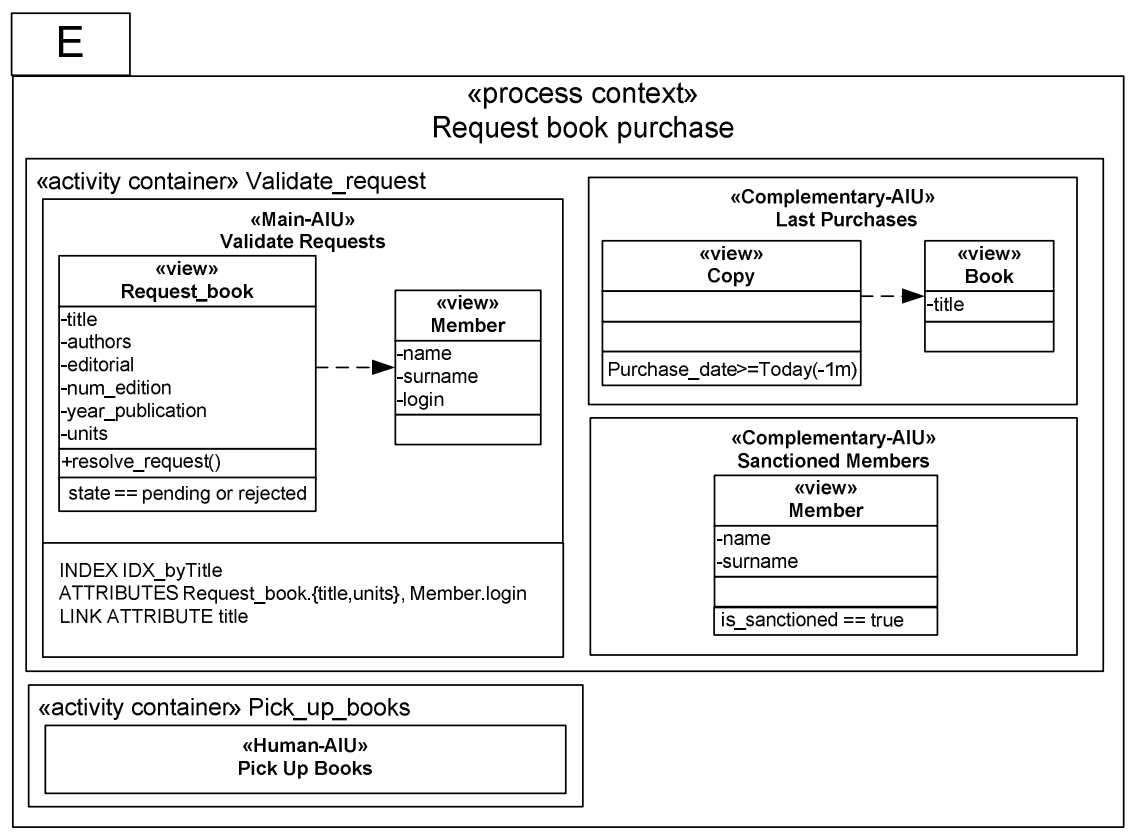

Figure C.10 RBP Process Context for the Secretary user

In addition, to avoid overloading the user interface with too many information related to book requests, we have also introduced an index. In this case, when the index gets activated just the 
attributes specified in the index definition are going to be displayed (see Figure C.11).

In this case, we are going to specify a presentation requirement associated to the "Validate_request" activity container. We want to handle multiple request instances at the same time. For this reason we have associated the List builder pattern to the activity container.

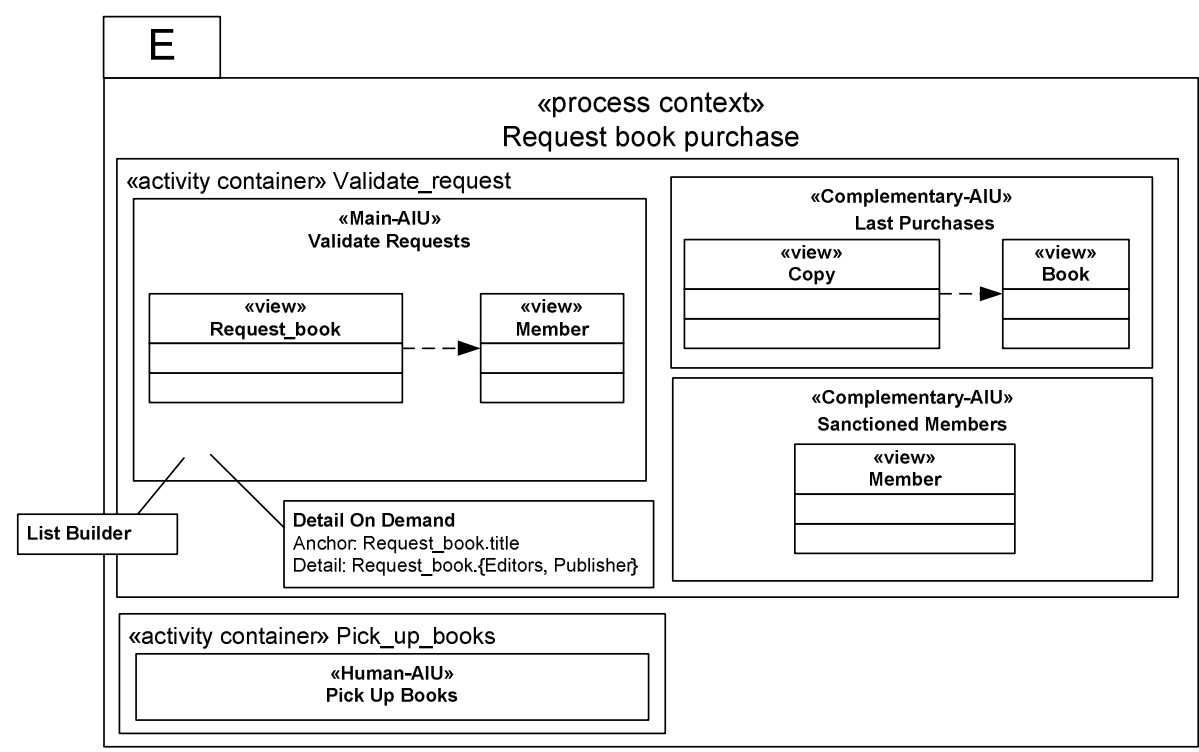

Figure C.11 RBP Process Context for the Secretary user (Presentation properties)

The application of this pattern allows the user solving multiple activity instances at the same time (see Figure C.12). The application of this pattern allows reducing the time invested in some tasks as well as improving the user experience by avoid the user completing each activity instance one by one. However, the application of this pattern does not mean that the user cannot 
proceed with each activity instance separately. In fact, this possibility is still provided to the user. In fact, by selecting the title of the book she can retrieve all the data associated to the particular activity instance.

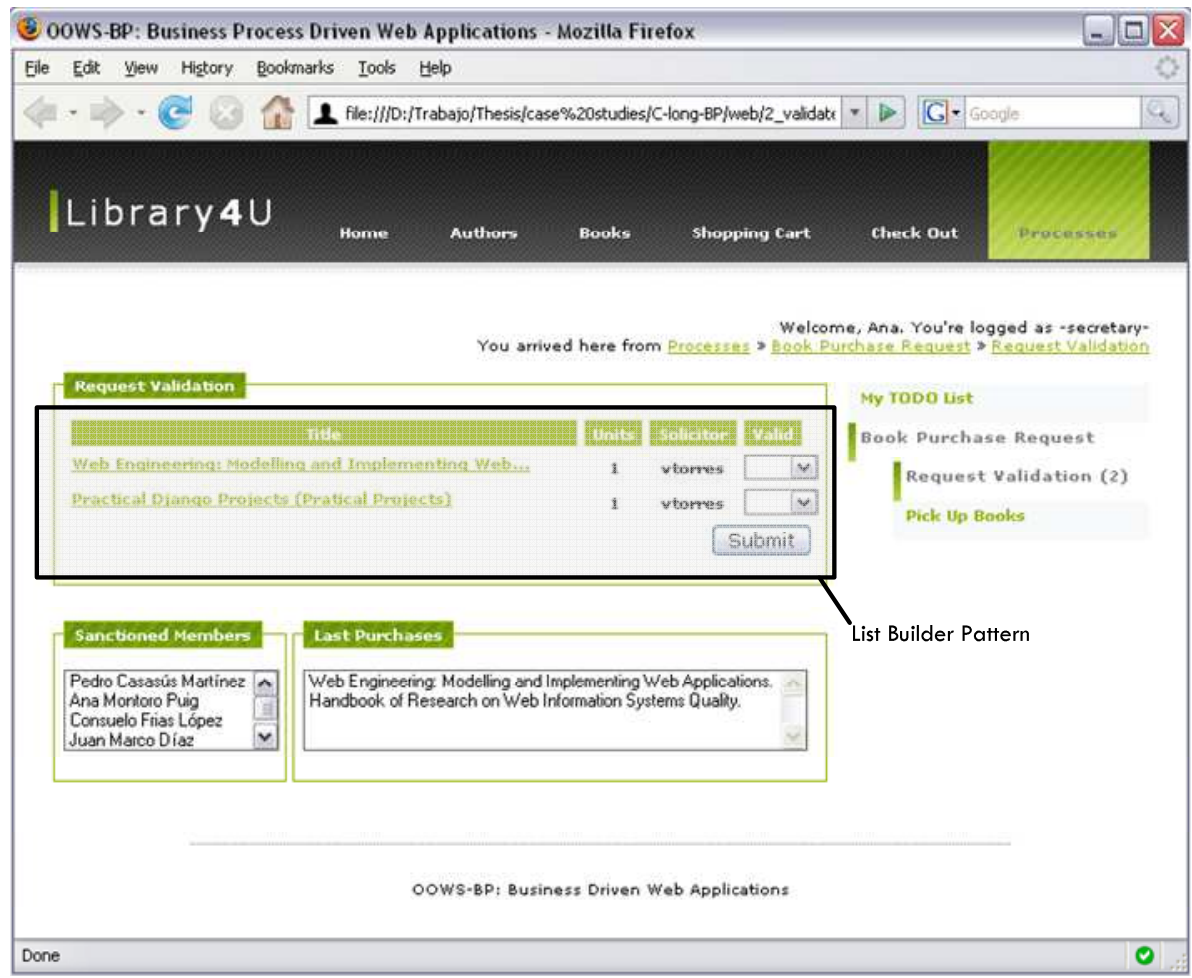

Figure C.12 List Build pattern applied to the Request Validation Activity Container

In addition, the Detail On Demand pattern has also been applied (see Figure C.13). In this case we have used this pattern to display more data related to a particular activity instance but without leaving the current page. This information is contained in the page but only shown when the cursor is positioned over the title link. 


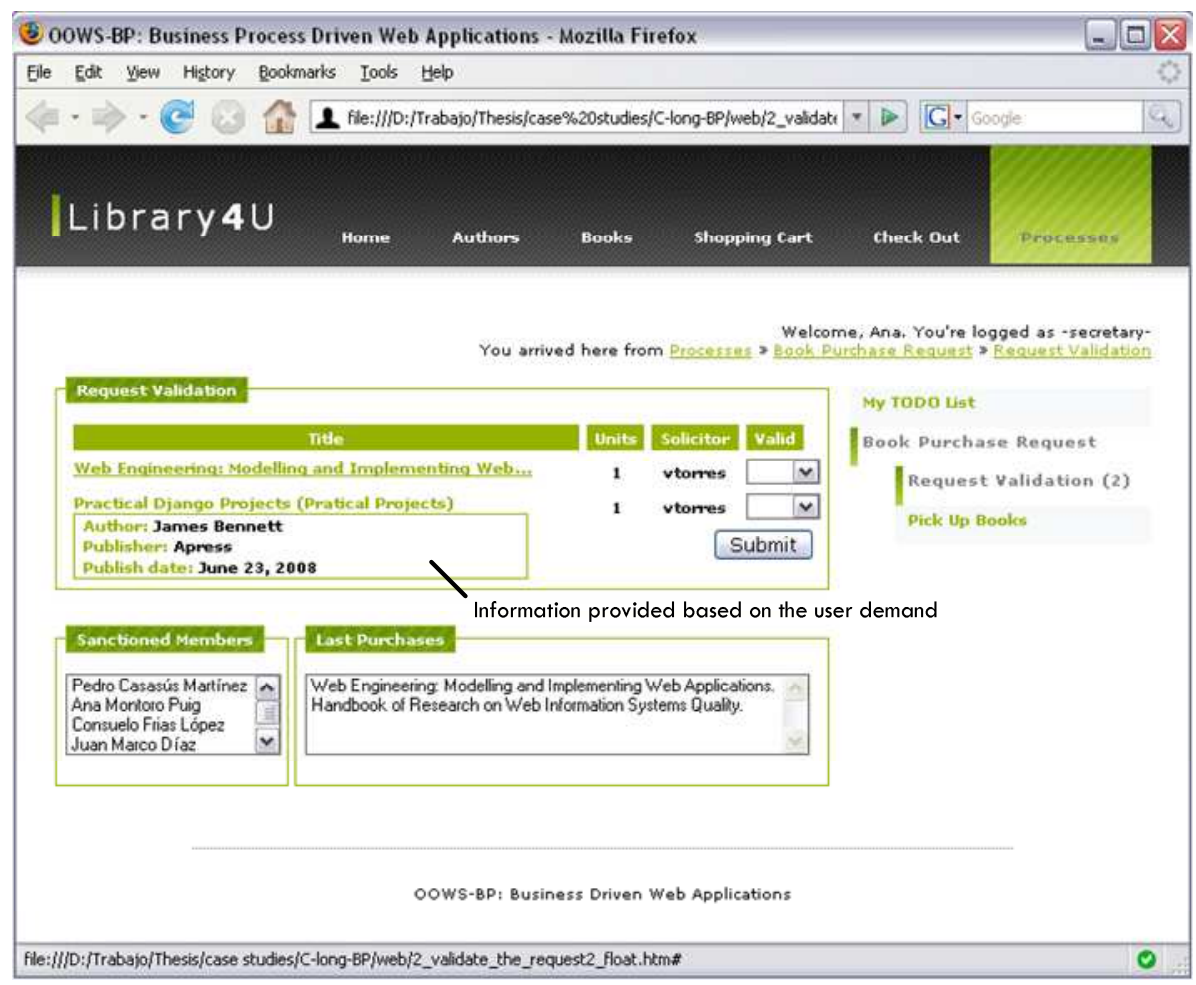

Figure C.13 Detail On Demand pattern applied to the Request Validation Activity Container

On the other hand, the Pick_up_books activity container represents a human activity and allows the secretary user to notify the process when this task has been completed. Figure C.14 shows the web page associated to this activity container. In this case, the web page simply provides information about the activity. 


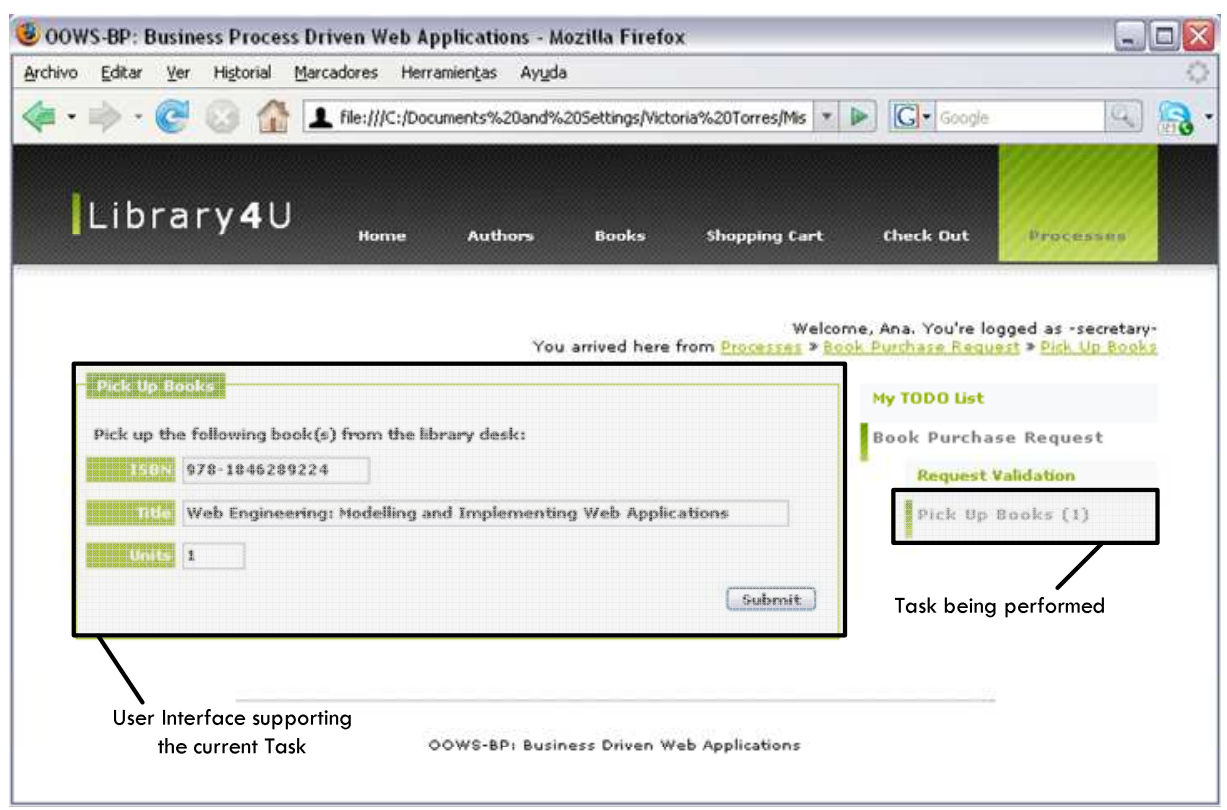

Figure C.14 Generated interface for the "Pick Up Books" activity

\section{C.5.3 Librarian Navigational Model}

\section{C.5.3.1Authoring-in-the-large}

Similarly to the secretary user type, the librarian inherits the navigational map defined for the member user type. Therefore, in this case we have to redefine the inherited process context as Figure C.15 shows.

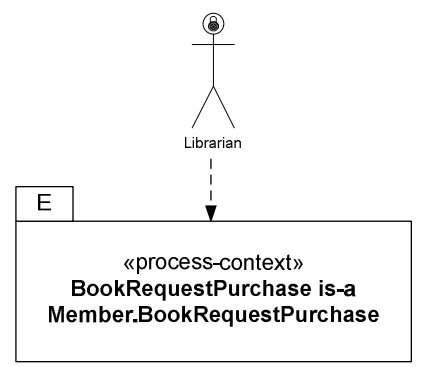

Figure C.15 Librarians Navigational Map 


\section{C.5.3.2Authoring-in-the-small}

In this case, the librarian user type has only one responsibility regarding the BPR-BP. This activity is related with the loan event where the system registers that this book copy has been lent to a particular user. For this reason, the Loan_book activity container includes the loan_copy operation of the copy class (see Figure C.16).

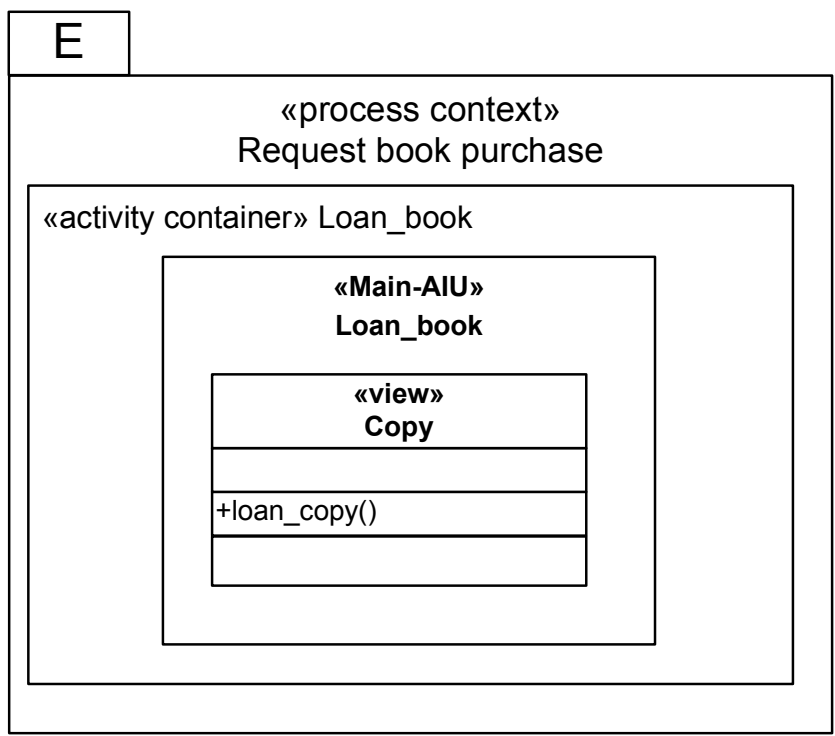

Figure C.16 Loan book Navigational Context

In this case, the librarian user is simply asked for certifying that the loan has been performed. For this reason the generated web interface associated to this activity container simply shows the information associated to the loan transaction which is the book copy and the applicant member (see Figure C.17). 


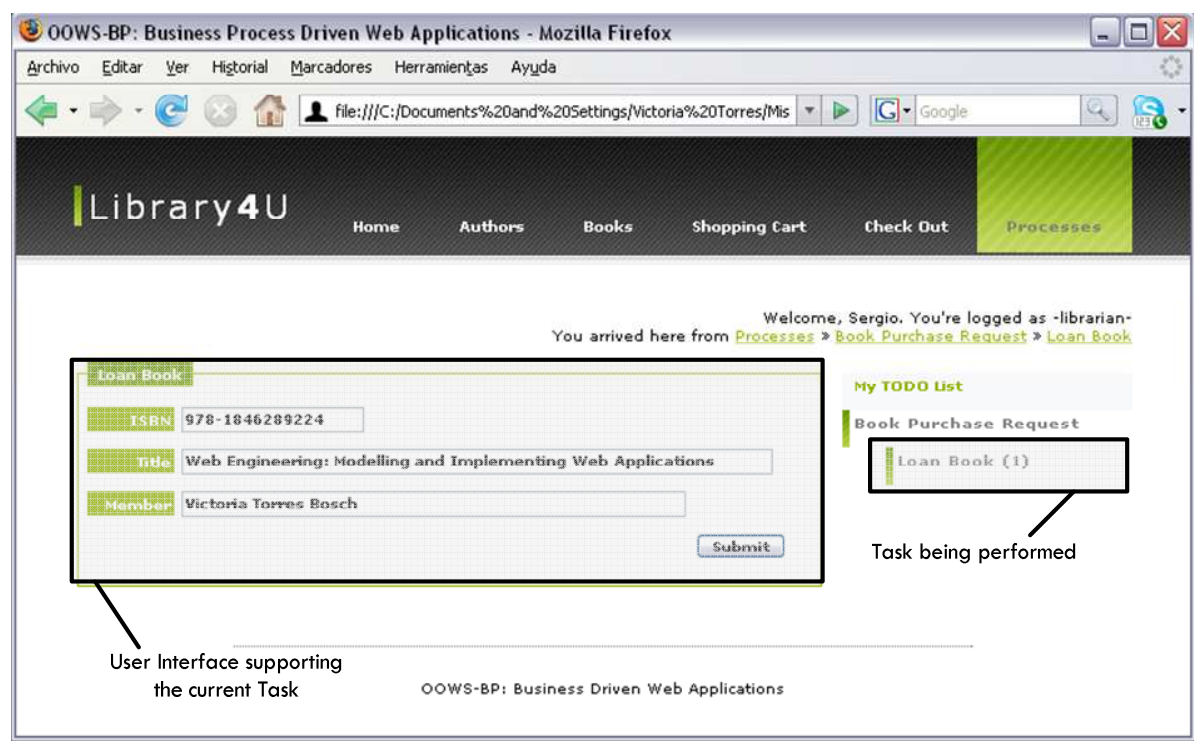

Figure C.17 Generated interface for the "Loan Book" activity 


\section{References}

Abran, A., Khelifi, A., Suryn, W., \& Seffah, A. (2003). Usability meanings and interpretations in iso standards. Software Quality Journal, 11(4), 325-338.

Agrawal, A., Amend, M., Das, M., Ford, M., Keller, C., Kloppmann, M., König, D., Leymann, F., Müller, R., Pfau, G., Plösser, K., Rangaswamy, R., Rickayzen, A., Patrick Schmidt, M. R., Trickovic, I., Yiu, A., \& Matthias, Z. (2007a). Wshumantask v1.0. Active Endpoints, Adobe, BEA Systems, IBM, Oracle and SAP AG.

Agrawal, A., Amend, M., Das, M., Ford, M., Keller, C., Kloppmann, M., König, D., Leymann, F., Müller, R., Pfau, G., Plösser, K., Rangaswamy, R., Rickayzen, A., Rowley, M., Schmidt, P., Trickovic, I., Yiu, A., \& Zeller, M. (2007b). Bpel4people v1.0. Active Endpoints, Adobe, BEA Systems, IBM, Oracle and SAP AG.

Andrews, T., Curbera, F., Dholakia, H., Goland, Y., Klein, J., Leymann, F., Liu, K., Roller, D., Smith, D., Thatte, S., Trickovic, I., \& Weerawarana, S. (2003). Business process execution language for web services version 1.1. 
Atterer, R., Schmidt, A., \& Hußmann, H. (2006). Extending web engineering models and tools for automatic usability validation. J. Web Eng., 5(1), 43-64.

Benatallah, B., Casati, F., Grigori, D., Nezhad, H. R. M., \& Toumani, F. (2005). Developing adapters for web services integration. In O. Pastor, \& J. F. e Cunha (Eds.) CAiSE, vol. 3520 of Lecture Notes in Computer Science, (pp. 415-429). Springer.

Berners-Lee, T. (1996). WWW: past, present, and future. 29, 6977.

Bozzon, A., Comai, S., Fraternali, P., \& Carughi, G. T. (2006). Conceptual modeling and code generation for rich internet applications. In D. Wolber, N. Calder, C. Brooks, \& A. Ginige (Eds.) ICWE, (pp. 353-360). ACM.

Brambilla, M. (2003). Extending hypertext conceptual models with process-oriented primitives. In I.-Y. Song, S. W. Liddle, T. W. Ling, \& P. Scheuermann (Eds.) ER, vol. 2813 of Lecture Notes in Computer Science, (pp. 246-262). Springer.

Brambilla, M., Celino, I., Ceri, S., Cerizza, D., Valle, E. D., \& Facca, F. M. (2006a). A software engineering approach to design and development of semantic web service applications. In I. F. Cruz, S. Decker, D. Allemang, C. Preist, D. Schwabe, P. Mika, M. Uschold, \& L. Aroyo (Eds.) International 
Semantic Web Conference, vol. 4273 of Lecture Notes in Computer Science, (pp. 172-186). Springer.

Brambilla, M., Ceri, S., Comai, S., \& Fraternali, P. (2006b). A case tool for modelling and automatically generating web serviceenabled applications. Int. J. Web Eng. Technol., 2(4), 354-372.

Brambilla, M., Ceri, S., Comai, S., Fraternali, P., \& Manolescu, I. (2003). Specification and design of workflow-driven hypertexts. In $W W W$ (Posters).

Brambilla, M., Ceri, S., Fraternali, P., \& Manolescu, I. (2006c). Process modeling in web applications. ACM Trans. Softw. Eng. Methodol., 15(4), 360-409.

Brambilla, M., Comai, S., Fraternali, P., \& Matera, M. (2008). Web Engineering: Modelling and Implementing Web Applications, chap. Designing Web Applications with Webml and Webratio, (pp. 221-261). Human-Computer Interaction Series. Springer London.

Burstein, M., Hobbs, J., Lassila, O., Mcdermott, D., Mcilraith, S., Narayanan, S., Paolucci, M., Parsia, B., Payne, T., Sirin, E., Srinivasan, N., \& Sycara, K. (2004). Owl-s: Semantic markup for web services. Website. 
Cáceres, P., de Castro, V., \& Marcos, E. (2004). Navigation modelling from a user services oriented approach. In ADVIS, (pp. 150-160).

Cachero, C., \& Gómez, J. (2002). Advanced conceptual modeling of web applications: Embedding operation interfaces in navigation design. In M. Celma, O. Pastor, N. J. Juzgado, \& J. J. Moreno-Navarro (Eds.) JISBD, (pp. 235-248).

Casteleyn, S. (2005). Designer Specified Self Re-organizing Websites,. Ph.D. thesis, Vrije Universiteit Brussel.

Ceri, S., Daniel, F., Facca, F. M., \& Matera, M. (2007). Modeldriven engineering of active context-awareness. World Wide Web, 104), 387-413.

Ceri, S., Fraternali, P., \& Bongio, A. (2000). Web modeling language (webml): a modeling language for designing web sites. In Proceedings of the 9th international World Wide Web conference on Computer networks : the international journal of computer and telecommunications netowrking, (pp. 137157). Amsterdam, The Netherlands, The Netherlands: NorthHolland Publishing Co.

Cunin, P.-Y., Greenwood, R. M., Francou, L., Robertson, I., \& Warboys, B. (2001). The pie methodology - concept and application. In V. Ambriola (Ed.) EWSPT, vol. 2077 of Lecture Notes in Computer Science, (pp. 3-26). Springer. 
Czarnecki, K., \& Helsen, S. (2003). Classification of model transformation approaches. In OOPSLA'O3 Workshop on Generative Techniques in the Context of Model-Driven Architecture.

de Assis, P. S., Schwabe, D., \& Nunes, D. A. (2006). Ashdm model-driven adaptation and meta-adaptation. In V. P. Wade, H. Ashman, \& B. Smyth (Eds.) AH, vol. 4018 of Lecture Notes in Computer Science, (pp. 213-222). Springer.

Distante, D. (2004). Reengineering legacy applications and web transactions: an extended version of the UWA transaction design model. Ph.D. thesis, University of Lecce, Italy.

Distante, D., Rossi, G., \& Canfora, G. (2007a). Modeling business processes in web applications: an analysis framework. In Y. Cho, R. L. Wainwright, H. Haddad, S. Y. Shin, \& Y. W. Koo (Eds.) SAC, (pp. 1677-1682). ACM.

Distante, D., Rossi, G., Canfora, G., \& Tilley, S. R. (2007b). A comprehensive design model for integrating business processes in web applications. Int. J. Web Eng. Technol., 3(1), 43-72.

Fialho, A. T. S., \& Schwabe, D. (2007). Enriching hypermedia application interfaces. In L. Baresi, P. Fraternali, \& G.-J. Houben (Eds.) ICWE, vol. 4607 of Lecture Notes in Computer Science, (pp. 188-193). Springer. 
Firesmith, D. G., \& Henderson-Sellers, B. (2001). The OPEN Process Framework. Addison-Wesley Longman.

Fons, J. (2008). OOWS: A Model Driven Method for the Development of Web Applications. Ph.D. thesis, Technical University of Valencia.

Fons, J., Pelechano, V., Pastor, O., Valderas, P., \& Torres, V. (2008). Web Engineering: Modelling and Implementing Web Applications, chap. Applying the OOWS Model-Driven Approach for Developing Web Applications. The Internet Movie Database Case Study, (pp. 65-108). Human-Computer Interaction Series. Springer London.

Garrigós, I., Gomez, J., \& Cachero, C. (2003). Modelling dynamic personalization in web applications. In ICWE, (pp. 472-475).

Garzotto, F., \& Perrone, V. (2007). Industrial acceptability of design methods: an empirical study. Journal of Web Engineering, 6, 73-96.

Ginige, A., \& Murugesan, S. (2001a). Guest editors' introduction: The essence of web engineering-managing the diversity and complexity of web application development. IEEE MultiMedia, $8(2), 22-25$. 
Ginige, A., \& Murugesan, S. (2001b). Guest editors' introduction: Web engineering an introduction. IEEE MultiMedia, 8(1), 1418.

Gómez, J., Cachero, C., \& Pastor, O. (2000). Extending a conceptual modelling approach to web application design. In B. Wangler, \& L. Bergman (Eds.) CAiSE, vol. 1789 of Lecture Notes in Computer Science, (pp. 79-93). Springer.

Hollingsworth, D. (1995). The workflow reference model. document number tc00-1003.

Hornung, T., Koschmider, A., \& Oberweis, A. (2007). Rule-based autocompletion of business process models. In CAiSE Forum.

Houben, G.-J. (2000). HERA: Automatically generating hypermedia front-ends. In EFIS, (pp. 81-88).

Houben, G.-J., Barna, P., Frasincar, F., \& Vdovjak, R. (2003). Hera: Development of semantic web information systems. In J. M. C. Lovelle, B. M. G. Rodríguez, L. J. Aguilar, J. E. L. Gayo, \& M. del Puerto Paule Ruíz (Eds.) ICWE, vol. 2722 of Lecture Notes in Computer Science, (pp. 529-538). Springer.

Houben, G.-J., Frasincar, F., Barna, P., \& Vdovjak, R. (2004). Modeling user input and hypermedia dynamics in hera. In N. Koch, P. Fraternali, \& M. Wirsing (Eds.) ICWE, vol. 3140 of Lecture Notes in Computer Science, (pp. 60-73). Springer. 
Houben, G.-J., van der Sluijs, K., Barna, P., Broekstra, J., , S. C., Fiala, Z., \& Frasincar, F. (2008). Web Engineering: Modelling and Implementing Web Applications, chap. HERA, (pp. 263301). Human-Computer Interaction Series. Springer London.

Jouault, F., \& Kurtev, I. (2006). On the architectural alignment of atl and qvt. In H. Haddad (Ed.) SAC, (pp. 1188-1195). ACM.

Kappel, G., Pröll, B., Reich, S., Retschitzegger, W., Grünbacher, P., Schwinger, W., Koch, N., Eichinger, C., Austaller, G., Hartl, A., Lauff, M., Lyardet, F., Mühlhäuser, M., Nussbaumer, M., Gaedke, M., Steindl, C., Ramler, R., Altmann, J., Ebner, A., Pröll, B., Werthner, H., Mayr, H., Engels, G., Lohmann, M., Wagner, A., Hitz, M., Leitner, G., Melcher, R., Kotsis, G., Wimmer, M., Kemper, A., Seltzsam, S., Behrendt, W., \& Arora, N. (2006). Web Engineering. The Discipline of Systematic Development of Web Applications. John Wiley \& Sons, Ltd.

Kloppmann, M., Koenig, D., Leymann, F., Pfau, G., Rickayzen, A., von Riegen, C., Schmidt, P., \& Trickovic, I. (2005). Ws-bpel extension for people - bpel4people.

Knapp, A., Koch, N., Zhang, G., \& Hassler, H.-M. (2004). Modeling business processes in web applications with argouwe. In T. Baar, A. Strohmeier, A. M. D. Moreira, \& S. J. Mellor (Eds.) UML, vol. 3273 of Lecture Notes in Computer Science, (pp. 69-83). Springer. 
Koch, N. (2001). Software Engineering for Adaptive Hypermedia Systems: Reference Model, Modeling Techniques and Development Process. Ph.D. thesis, Ludwig-MaximiliansUniversity Munich, Germany.

Koch, N. (2006). Transformation techniques in the model-driven development process of uwe. In ICWE '06: Workshop proceedings of the sixth international conference on Web engineering, (p. 3). New York, NY, USA: ACM.

Koch, N. (2007). Classification of model transformation techniques used in uml-based web engineering. IET Software Journal, 1(3), 98-111.

Koch, N., Knapp, A., Zhang, G., \& Baumeister, H. (2008). Web Engineering: Modelling and Implementing Web Applications, chap. Uml-Based Web Engineering, (pp. 157-191). HumanComputer Interaction Series. Springer London.

Koch, N., \& Kraus, A. (2002). The expressive power of uml-based engineering. In Second International Workshop on Web Oriented Software Techonlogy (CYTED), (pp. 105-119).

Koch, N., Kraus, A., Cachero, C., \& Meliá, S. (2004). Integration of business processes in web application models. J. Web Eng., 3(1), 22-49. 
Kraus, A., Knapp, A., \& Koch, N. (2007). Model-driven generation of web applications in uwe. In 3rd International Workshop on Model-Driven Web Engineering. MDWE 2007 3rd International Workshop on Model-Driven Web Engineering (Proceedings) CEUR-WS, Vol 261, July 2007.

Lima, F., \& Schwabe, D. (2003). Application modeling for the semantic web. In Web Congress, 2003. Proceedings. First Latin American, (pp. $93-$ 102).

Marcos, E., Cáceres, P., \& Castro, V. D. (2004). An approach for navigation model construction from the use cases model. CAiSE Forum. Held in conjunction with the 16th Conference On Advanced Information Systems Engineering.

Marcos, E., de Castro, V., \& Vela, B. (2003a). Representing web services with uml: A case study. In ICSOC, (pp. 17-27).

Marcos, E., Vela, B., \& Cavero, J. M. (2003b). A methodological approach for object-relational database design using uml. Software and System Modeling, 2(1), 59-75.

Mayhew, D. J. (1992). Principles and guidelines in software user interface design. Upper Saddle River, NJ, USA: Prentice-Hall, Inc.

Mendes, E., \& Mosley, N. (Eds.) (2006). Web Engineering: Springer. 
Miller, J., \& Mukerji, J. (2003). MDA Guide Version 1.0.1. Tech. Rep. omg/03-06-01, Object Management Group (OMG).

Murugesan, S., \& Ginige, A. (2001). Web engineering: Introduction and perspectives - overview. In S. Murugesan, \& Y. Deshpande (Eds.) Web Engineering, vol. 2016 of Lecture Notes in Computer Science, (pp. 1-2). Springer.

Nielsen, J. (1999). Designing Web Usability. Peachpit Press.

OMG (2004). Unified modeling language specification: Version 2, revised final adopted specification (ptc/04-10-02).

OMG (2005a). MOF QVT Final Adopted Specification.

OMG (2005b). Software process engineering metamodel specification (SPEM), v1.1. Tech. rep., Object Manament Group.

OMG (2006). Business process modeling notation (bpmn) specification, omg final adopted specification. dtc/06-02-01.

OMG (2007a). Business process definition metamodel (bpdm), beta 1.

OMG (2007b). Business process model and notation (bpmn) 2.0 request for proposal, omg document: Bmi/2007-06-05. 
Ouyang, C., van der Aalst, W. M., Dumas, M., \& ter Hofstede, A. H. (2006). From business process models to processoriented software systems: The bpmn to bpel way. Tech. rep., Queensland University of Technology.

Pastor, O., Gómez, J., Insfrán, E., \& Pelechano, V. (2001). The OOmethod approach for information systems modeling: from object-oriented conceptual modeling to automated programming. Inf. Syst., 26(7), 507-534.

Paternò, F., Mancini, C., \& Meniconi, S. (1997). Concurtasktrees: A diagrammatic notation for specifying task models. In INTERACT, (pp. 362-369).

Pressman, R. S., Lewis, T. G., Adida, B., Ullman, E., DeMarco, T., Gilb, T., Gorda, B. C., Humphrey, W. S., \& Johnson, R. (1998). Can internet-based applications be engineered? IEEE Software, 15(5), 104-110.

Quintero, R. (2008). Desarrollo Dirigido por Modelos de Aplicaciones Web que integran Datos y Funcionalidad a partir de Servicios Web. Ph.D. thesis, Technical University of Valencia.

Reenskaug, T. (1979). Models - views - controllers. Tech. rep., Technical Note, Xerox Parc. 
Reenskaug, T. (2003). The model-view-controller (mvc) its past and present.

Rojas, G. (2008). Modelling Adaptive Web Applications in OOWS. Ph.D. thesis, Technical University of Valencia.

Roman, D., Keller, U., Lausen, H., de Bruijn, J., Lara, R., Stollberg, M., Polleres, A., Feier, C., Bussler, C., \& Fensel, D. (2005). Wsmo - web service modeling ontology. In DERI Working Draft 14, vol. 1, (pp. 77-106). Digital Enterprise Research Institute (DERI), BG Amsterdam: IOS Press.

Rossi, G., \& Schwabe, D. (2008). Web Engineering: Modelling and Implementing Web Applications, chap. Modeling and Implementing Web Applications with Oohdm, (pp. 109-155). Human-Computer Interaction Series. Springer London.

Schmid, H. A., \& Rossi, G. (2004). Modeling and designing processes in e-commerce applications. IEEE Internet Computing, $8(1), 19-27$.

Schmidt, D. C. (2006). Model-driven engineering. IEEE Computer, 39(2), 25-31.

Schwabe, D., \& Rossi, G. (1998). An object oriented approach to web-based applications design. Theor. Pract. Object Syst., 4(4), 207-225. 
Schwabe, D., Szundy, G., Silva de Moura, S., \& Lima, F. (2004). Design and implementation of semantic web applications. In WWW Workshop on Application Design, Development and Implementation Issues in the Semantic Web.

Selic, B. (2003). The pragmatics of model-driven development. IEEE Software, 20(5), 19-25.

Sousa, K. S., Filho, H. M., \& Vanderdonckt, J. (2007). Towards method engineering of model-driven user interface development. In M. Winckler, H. Johnson, \& P. A. Palanque (Eds.) TAMODIA, vol. 4849 of Lecture Notes in Computer Science, (pp. 112-125). Springer.

S.T., M., \& G.F., S. (1995). Design and natural science research on information technology. Decision Support Systems, 15, 251266(16).

Torres, V., Giner, P., \& Pelechano, V. (2007). Modeling ubiquitous business process driven applications. In J. Eder, S. L. Tomassen, A. L. Opdahl, \& G. Sindre (Eds.) CAiSE Forum, vol. 247 of CEUR Workshop Proceedings. CEUR-WS.org.

Torres, V., Pelechano, V., Ruíz, M., \& Valderas, P. (2005). A model driven approach for the integration of external functionality in web applications. the travel agency system. In Proceedings of the 1st Workshop on Model-driven Web Engineering (MDWE 2005). 
Troyer, O. D., \& Casteleyn, S. (2004). Designing localized web sites. In X. Zhou, S. Y.W. Su, M. P. Papazoglou, M. E. Orlowska, \& K. G. Jeffery (Eds.) WISE, vol. 3306 of Lecture Notes in Computer Science, (pp. 547-558). Springer.

Troyer, O. D., Casteleyn, S., \& Plessers, P. (2008). Web Engineering: Modelling and Implementing Web Applications, chap. WSDM: Web Semantics Design Method, (pp. 303-351). Human-Computer Interaction Series. Springer London.

Troyer, O. D., \& Leune, C. J. (1998). Wsdm: A user centered design method for web sites. Computer Networks, 30(1-7), 8594.

UWA Consortium (2002). Ubiquitous web applications. In Proceedings of e2002 eBusiness and eWork Conference.

Vaishnavi, V., \& Kuechler, W. (2004). Design research in information systems, last accessed on September 9th, 2004, revision from February 20th.

Valderas, P. (2008). A Requirements Engineering Approach for the Development of Web Applications. Ph.D. thesis, Technical University of Valencia.

Vallecillo, A., Koch, N., Cachero, C., Comai, S., Fraternali, P., Garrigós, I., Gómez, J., Kappel, G., Knapp, A., Matera, M., Meliá, S., Moreno, N., Pröll, B., Reiter, T., Retschitzegger, W., 
Rivera, J. E., Schauerhuber, A., Schwinger, W., Wimmer, M., \& Zhang, G. (2007). Mdwenet: A practical approach to achieving interoperability of model-driven web engineering methods. In Proceedings of the 3rd International Workshop on Model-Driven Web Engineering. MDWE 2007.

Valverde, F., Valderas, P., Fons, J., \& Pastor, O. (2007). A mdabased environment for web applications development: From conceptual models to code. In 6th International Workshop on Web-Oriented Software Technologies (IWWOST). Como (Italy).

van der Sluijs, K., Houben, G.-J., Broekstra, J., \& Casteleyn, S. (2006). Hera-s: web design using sesame. In D. Wolber, N. Calder, C. Brooks, \& A. Ginige (Eds.) ICWE, (pp. 337-344). ACM.

van Welie, M. Patterns in interaction design. http://www.welie.com/.

WfMC (1999). Terminology \& glossary. document number wfmc-tc1011. document status - issue 3.0.

White, S. A. (2005). Using bpmn to model a bpel process. Object Management Group. BPMN Articles. 


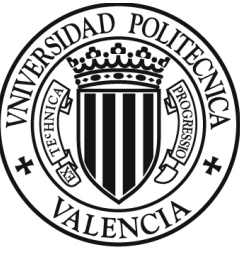

UNIVERSIDAD POLITECNICA DE VALENCIA 